\title{
Neural Control of Respiration by the Retrotrapezoid Nucleus
}

Tyler Matthew Basting

Dover Plains, NY

B.S., Lafayette College, 2012

A Dissertation presented to the Graduate Faculty of the University of Virginia in Candidacy for the Degree of Doctorate of Philosophy

\section{Department of Neuroscience}

University of Virginia

July, 2016

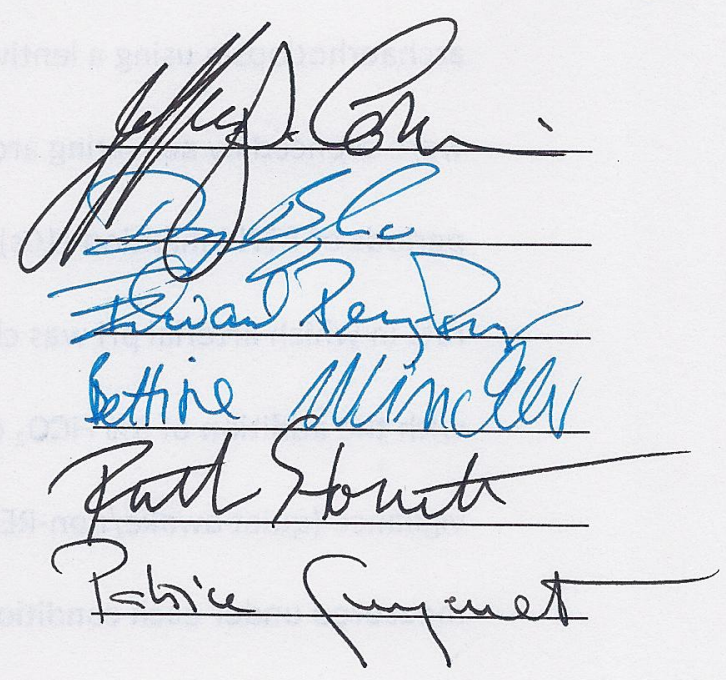




\section{Abstract}

Rationale and objectives: Central respiratory chemoreceptors (CRCs) detect brain $\mathrm{PaCO}_{2}$ and adjust lung ventilation to maintain $\mathrm{PaCO}_{2}$ and $\mathrm{pH}$ constant regardless of the absolute level of lung ventilation. They perform this function in cooperation with the carotid bodies, sensory organs that respond to hypoxia in a pH-dependent manner. The existence of CRCs has been known for over a century but their cellular nature and location have remained highly controversial until recently. The retrotrapezoid nucleus (RTN), a collection of $\sim 2000$ glutamatergic neurons in rats that are activated by hypercapnia in vivo and by acidification in slices, had been identified as a CRC candidate just prior to my PhD work. My first objective was to seek additional and critical evidence that RTN neurons are CRCs by determining whether these neurons stimulate breathing in proportion to arterial $\mathrm{PCO}_{2}$ in intact unanesthetized rats. Having verified that this was the case I tested a logical corollary of this theory namely that RTN is silenced under hypoxic conditions due to respiratory alkalosis. Then I proceeded to test whether RTN neurons sustain breathing automaticity during sleep. Finally, I tested whether RTN compensates for the lack peripheral chemoreceptor input.

Methods: RTN neurons were bilaterally transduced to express the proton pump archaerhodopsin using a lentiviral vector. Using anesthetized rats, I confirmed that RTN neurons were silenced by activating archaerhodopsin with green light. I examined the effect of short periods of RTN inhibition (10s) on breathing (plethysmography), EEG and neck EMG in conscious rats in which arterial $\mathrm{pH}$ was changed by exposing the animals to various $\mathrm{FiO}_{2}$ levels alone or with the addition of $3 \% \mathrm{FiCO}_{2}$ or acetazolamide (ACTZ). Rats were studied in different states of vigilance (quiet awake/non-REM/REM). Arterial blood gases $\left(\mathrm{PaO}_{2}, \mathrm{PaCO}_{2}\right), \mathrm{pHa}$ and $\mathrm{HCO}_{3}$ were measured under each condition. 
Results: RTN inhibition (bilateral) reduced breathing frequency and amplitude in direct proportion to arterial plasma $\mathrm{pH}(\mathrm{pHa})$ below a threshold of 7.53. Above this level, RTN inhibition had no effect suggesting that the nucleus was silent. In normoxia, RTN inhibition reduced breathing equally during non-REM sleep and quiet wake. By contrast RTN inhibition had very little effect on breathing during REM sleep.

Conclusions: RTN drives breathing in direct proportion to arterial $\mathrm{PCO}_{2}$ in intact unaesthetized rats, consistent with the theory that these neurons are CRCs. RTN neurons are silent above pHa 7.5 and increasingly active below this value. RTN regulates breathing automaticity about equally during non-REM sleep and quiet waking, conditions under which the respiratory pattern generator is autorhythmic. By contrast, RTN has a much reduced influence on breathing during REM sleep, consistent with the known reduction of the chemoreflex during this stage of sleep. Hyperoxia activates RTN, which maintains ventilation at control level (normoxia) despite the presumed loss of carotid body input. Finally we demonstrate that RTN and the carotid bodies can regulate breathing independently of each other. 
Acknowledgements: First and foremost I would like to thank my advisor Patrice Guyenet. His wealth of knowledge, expertise in the field of respiration and positive attitude toward basic research has served to inspire and excite me throughout my graduate career. Patrice set up an environment that provided a young researcher with support and also allowed a healthy amount of independence. He has encouraged me to apply for grants, present at conferences, learn statistics and more, helping me build a strong resumé and publishing record. I would like to thank the core members of the lab during my tenure including Dr. Ruth Stornetta, Dr. Steve Abbott, Dr. Peter Burke, the future man Dr. Chikara Abe, Dr. lan Wenker, Ben Holloway and Ken Viar for surgery skills, science advice, life advice, wanted/unwanted music advice and so much more. Together, they made a dynamic group of personalities that provided a stimulating and encouraging atmosphere that will never be forgotten. I want to thank my committee members Dr. Bettina Winckler, Dr. Doug Bayliss, Dr. Jeff Corwin, Dr. Ed Perez-Reyez and Dr. Ruth Stornetta for having confidence in me and giving different perspectives on science. Finally, I want to thank my family and friends for their relentless support through my time as a student, past, present and future. Thank you Donna and Tim Basting for the love and support through all phases of my life, you have been essential to my academic and personal development! 


\section{Table of Contents}

Abstract

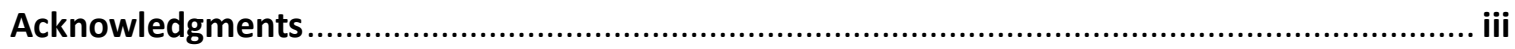

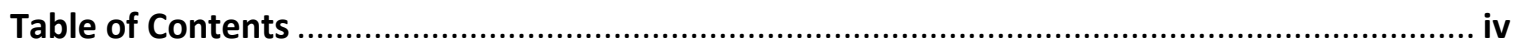

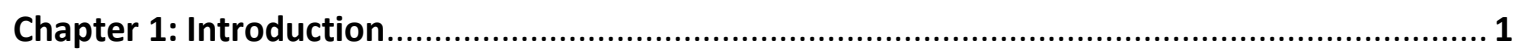

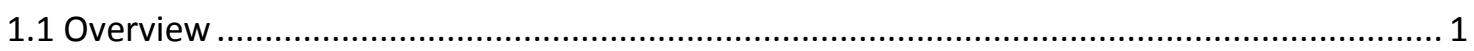

1.2 Definitions of Central Chemoreception and the Retrotrapezoid Nucleus (RTN) .................. 3

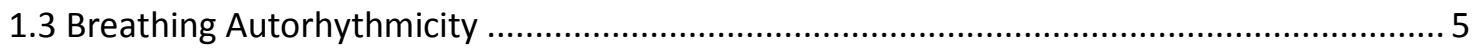

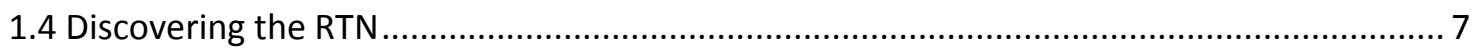

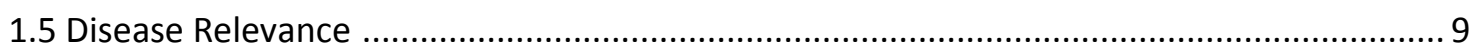

1.6 Location and Function of Carotid Bodies .................................................................... 10

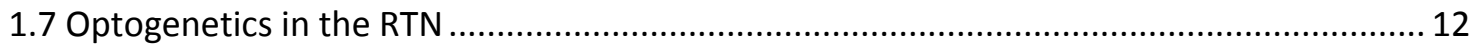

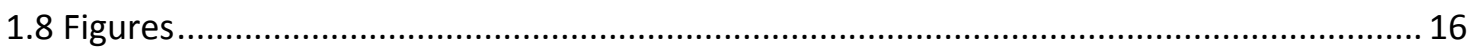

Chapter 2: Hypoxia silences the retrotrapezoid nucleus respiratory chemoreceptors via

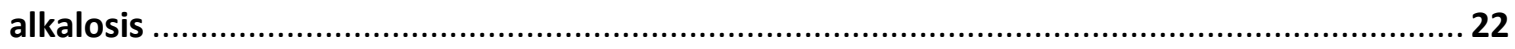

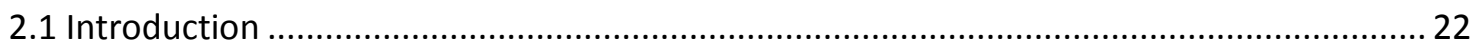

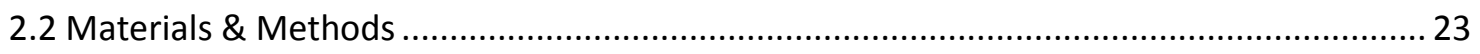

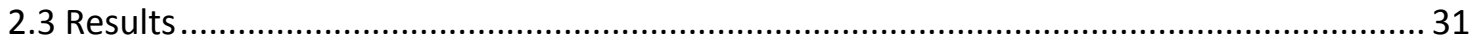

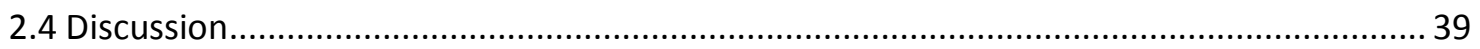

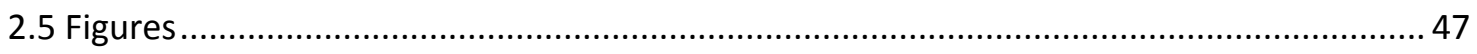

Chapter 3: State-dependent control of breathing by the retrotrapezoid nucleus ....................74

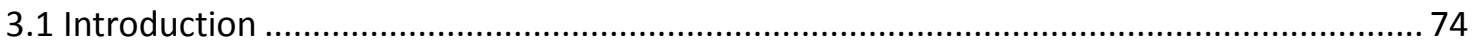

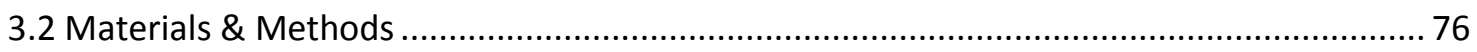

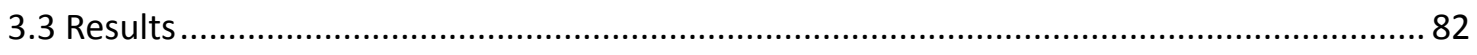

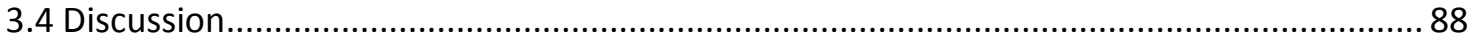

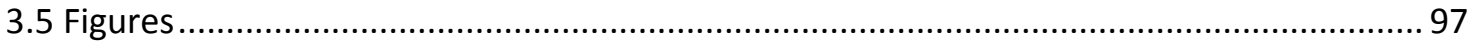

Chapter 4: Is plasticity within the retrotrapezoid nucleus responsible for the recovery of the PaCO2 set-point after carotid body denervation in rats? ...................................................... 118

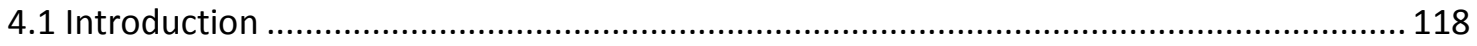

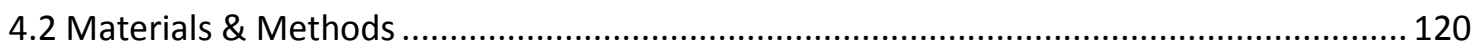




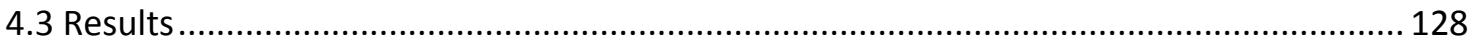

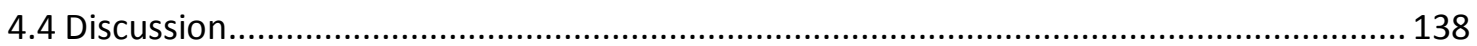

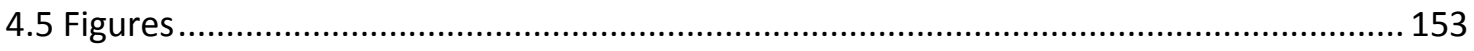

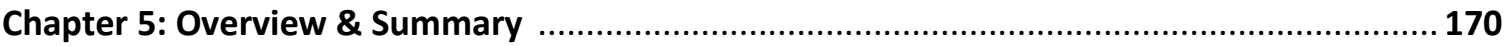

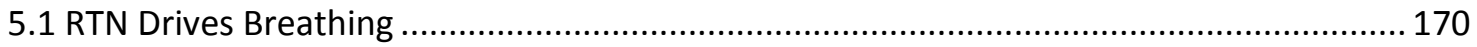

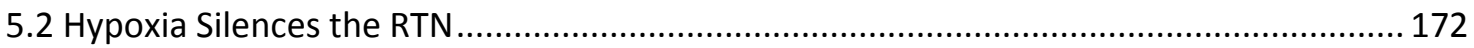

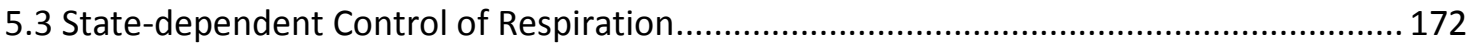

5.4 RTN's Role after Peripheral Chemoreceptor Denervation................................................. 174

5.5 Is Central Plasticity Responsible for Recovery After CBD ................................................ 175

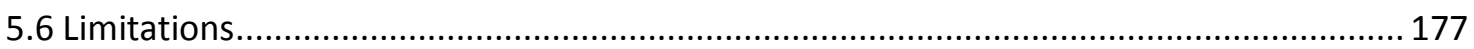

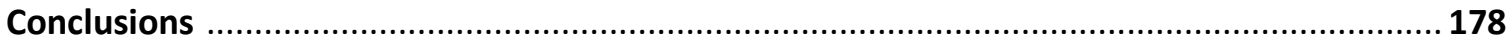

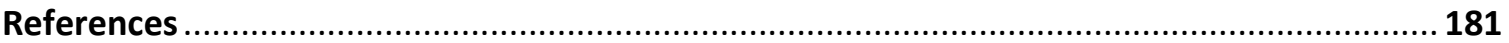




\section{Chapter 1: Introduction}

\subsection{Overview}

Lung ventilation in mammals is highly labile to enable vocalization/speech, chewing and swallowing, coughing and, at the same time, precisely regulated to maintain the balance between and the metabolic production of $\mathrm{CO}_{2}$ and its excretion by the lungs. Gas exchange is done by the alveoli in the lungs through diffusion, inhaling $\mathrm{O}_{2}$ rich air and exhaling air loaded with $\mathrm{CO}_{2}$. In order to meet the changing metabolic demands mammals have developed a system that maintains a relatively constant alveolar and arterial blood gas acid-base status. Although ventilation rate can vary widely depending on metabolic demand (sleep, wake, thermogenesis, exercise etc.) the partial pressure of arterial $\mathrm{CO}_{2}\left(\mathrm{PaCO}_{2}\right)$ remains surprisingly stable. Medullary rhythm/pattern generators, sensory inputs and motor outputs to respiratory musculature of the upper airway, chest and abdominal walls maintain the $\mathrm{PaCO}_{2}$ and $\mathrm{PaO}_{2}$. In the past three decades an extraordinary amount of progress has been made in the basic science of the neuronal network underlying ventilatory control. In this dissertation I briefly summarize a few of these findings and how we have made progress in the area of central and peripheral chemoreception.

Central respiratory chemoreception, the process by which the brain senses $\mathrm{pH} / \mathrm{PaCO}_{2}$ to regulate breathing, has been studied for over a century (Gesell et al., 1940; Mitchell et al., 1963b; Konig \& Seller, 1991; Severinghaus, 1998). Central chemoreceptors detect brain extracellular $\mathrm{PaCO}_{2} / \mathrm{H}+$ and activate breathing in proportion to the level of acidification, thereby contributing to $\mathrm{PaCO}_{2}$ homeostasis. $\mathrm{CO}_{2}$ is converted to carbonic acid $\left(\mathrm{H}_{2} \mathrm{CO}_{3}\right)$ by carbonic anhydrase which is in equilibrium with bicarbonate $\left(\mathrm{HCO}_{3}\right)$ and $\mathrm{H}+$. Therefore any increase in the metabolic production of $\mathrm{CO}_{2}$ tends to acidify the blood and, by extension, the brain extracullular 
fluid because $\mathrm{CO}_{2}$ is freely diffusible. It is this acidification that the central respiratory chemoreceptors (CRCs) sense. The CRCs influence respiration through their projections to the central pattern generator (CPG) where the rate and amplitude of respiration is encoded (Nattie, 2012; Guyenet et al., 2013a).

The retrotrapezoid nucleus (RTN) is postulated as a primary CRC and responsible for fine control of $\mathrm{PaCO}_{2}$ stabilization (Guyenet, 2014b). The effects of the CRCs, specifically the RTN, influence breathing through sensing $\mathrm{pH}$, integrating salient information and projecting to brain regions responsible for creating the respiratory rhythm. To understand this network the RTN has been studied three ways. First, molecular biology methods have been used to find which receptors are key players in sensing the $\mathrm{PaCO}_{2} / \mathrm{pH}$. Next, in vitro work has been done on both isolated RTN neurons and with surrounding cells (glia, neurons and microvasculature). Finally, work has been done in vivo to investigate interactions of the RTN with the respiratory network.

The most pertinent past discoveries include that the RTN neurons are excitatory, glutamatergic neurons that are robustly activated by $\mathrm{CO}_{2}$ in vitro and in vivo (Stornetta et al., 2006; Guyenet et al., 2012). However these effects caused by $\mathrm{CO}_{2}$ are complex. Excitation could be caused by direct effects of acid, produced by $\mathrm{CO}_{2}$ being rapidly converted to carbonic acid and then a balance between bicarbonate and $\mathrm{H}+$, on the neurons or paracrine effects of $\mathrm{CO}_{2}$ on the vasculature and astrocytes (Gourine et al., 2010; Gourine \& Kasparov, 2011). However, when isolated, the RTN neurons remain extremely sensitive to $\mathrm{pH}$ and respond with high levels of activation supporting the idea that this population is intrinsically chemosensitive (Wang et al., 2013c). Finally, the RTN's projections further strengthen its hypothesized role in respiratory chemoreception. It is exclusively linked to the respiratory column with massive projections to the central pattern generator (CPG), areas of the brainstem that control the muscle movements 
involved in inspiration and expiration, influencing both frequency and amplitude of respiration (Guyenet et al., 2013a).

\subsection{Definitions of Central Chemoreception and the Retrotrapezoid Nucleus (RTN)}

The all-encompassing definition of central chemoreception is how the brain senses, integrates and maintains fine control over $\mathrm{PaCO}_{2}$ in the body. The field of central chemoreception has progressed rapidly recently because of novel discoveries that examine the cellular and molecular underpinnings of these chemoreceptive areas. It has only been very recently, 2015, that potential ligands and receptors that sense the acidification of the RTN's local environment have been discovered. Two mechanisms for sensing $\mathrm{CO}_{2}$ were found in RTN neurons. TASK-2, a pH sensitive $\mathrm{K}+$ channel, which is expressed almost exclusively in the RTN relative to other CNS structures (Reyes et al., 1998; Gestreau et al., 2010) and the protonactivated G-protein coupled receptor GPR-4 (Ludwig et al., 2003; Liu et al., 2010). Dr. Doug Bayliss's group found that knock down of the K+ channel and the G-protein coupled receptor attenuates the $\mathrm{pH}$ sensitivity in vitro and nearly abolishes the hypercapnic ventilatory reflex, the respiratory reflex that alters breathing to normalize $\mathrm{PaCO}_{2}$, in vivo (Figure 2) (Gestreau et al., 2010; Wang et al., 2013a; Kumar et al., 2015). Other ligand candidates that may activate chemosensitive areas include molecular $\mathrm{CO}_{2}$ and bicarbonate in these finely $\mathrm{pH}$ tuned areas of the brain (Huckstepp \& Dale, 2011; Guyenet, 2014a).

In addition to the possibility of a combination of ligands and potential receptors, there has been work done on the types of cells detecting change in $\mathrm{pH}$. Both neuronal and glial populations have been shown to be $\mathrm{pH}$ sensitive (Gourine et al., 2009; Erlichman et al., 2010; Gourine et al., 2010; Huckstepp et al., 2010; Kasymov et al., 2013). Glia cells (astrocytes, astroglia) near the ventral surface of the brainstem have been shown to be $\mathrm{pH}$ sensitive and 
influence central respiratory chemoreceptors through purinergic (ATP) signaling in a paracrine type manner (Erlichman et al., 2010; Kasymov et al., 2013). This makes the neurons and glia both potential central chemoreceptor (CCR) candidates. These CCR's do not always project to or directly influence the central respiratory column but instead affect central respiratory chemoreceptors (CRC's). CRC's are unique populations of cells that both detect changes in $\mathrm{PaCO}_{2}$ and synapse onto the respiratory column. These two cell populations (CCR's and CRC's) often overlap such as in the case of the RTN. Characterizing these populations is difficult because every cell is $\mathrm{pH}$ sensitive to some degree and finding the cells that are both extremely $\mathrm{pH}$ sensitive and possess relevant connections to respiratory areas of the brain remains challenging.

The RTN is defined as a group of chemosensitive neurons that are glutamatergic, Phox $2 \mathrm{~b}$-positive and tyrosine-hydroxylase-negative. The neurons are located bilaterally on the ventral surface of the brainstem, caudal to the trapezoid bodies, numbering $\sim 1,000$ per side in rat, 400 per side in mouse (Stornetta et al., 2006; Lazarenko et al., 2009). The location, type of mRNA and enzyme expression was found by a combination of techniques. Electrophysiological recordings and juxtacellular labelling of individual RTN neurons were done in anesthetized rats. Detection of biochemical markers through immunohistochemistry and in situ hybridization were done afterwards. The biotinamide introduced by juxtacellular labeling expressed throughout the neurons provided information on cell structure (Pinault, 1996). RTN neurons are now known to have long dendrites that extend throughout the marginal layer of the ventral surface of the medulla oblongata where they sense blood/tissue $\mathrm{pH}$ at both the ventral surface of the brain and the internal environment (Mulkey et al., 2004). 
The RTN remains a top central respiratory chemoreceptor candidate, meaning it is both inherently sensitive to changes in $\mathrm{pH}$ and directly project to the central respiratory pattern generator, for several reasons. First, RTN neurons remain $\mathrm{pH}$ sensitive in vitro displaying intrinsic $\mathrm{pH}$ sensitivity when isolated. In coronal slices of the neonate rat brainstem acidification causes excitation even in the presence of glutamatergic, glycinergic and GABAergic ionotropic blockers plus PPADS (blocks ATP receptors) (Mulkey et al., 2004; Lazarenko et al., 2009). When neonate RTN neurons are isolated in a dish their pH sensitivity remains (Wang et al., 2013c). When RTN neurons are inhibited under normoxic conditions in conscious rats both breathing frequency and tidal volume decrease showing that these neurons are necessary to the drive to breathe (Basting et al., 2015). This information in combination with their connectome provides evidence that the RTN is important in integrating many $\mathrm{pH}$ sensitive areas of the brain and plays an important role in the drive to breathe. RTN neurons receive an abundance of projections from orexinergic, serotonergic and locus coeruleus neurons (other CCR's) and send projections to the respiratory column, the area that creates the respiratory rhythm (described below) (Doi \& Ramirez, 2008, 2010; Guyenet, 2014a).

\subsection{Breathing Autorhythmicity}

The generation of regular automatic breathing movements as observed in quiet awake or non-REM sleep is produced by constant back and forth between contraction and relaxation of pump and airway muscles. This muscle activity, when not being controlled through cortical areas of the brain, is determined by pacemaker neurons located in the preBötzinger complex working with multiple brainstem regions that together are called the respiratory column. This column is mainly comprised of four different parts, the Bötzinger complex, the preBötzinger complex, the 
rostral ventral respiratory group ( $\mathrm{VVRG}$ ) and the caudal ventral respiratory group (cVRG). Note that the respiratory column does not include the RTN because these areas can generate the respiratory rhythm without it but the vast majority of RTN projections end in one of these four areas.

Breathing consists of three phases, inspiration, post-inspiration and expiration. In order to maintain eupnea (normal patterned breathing) the interconnected respiratory column must function as a unit (Figure 3). Inspiration is driven by both the rVRG and the preBötzinger complex. The rVRG contains bulbospinal inspiratory premotor neurons that project to and activate motor neurons. These motor neurons innervate the muscles of the diaphragm. The preBötzinger complex provides the rhythm generation that rVRG premotor neurons use to coordinate patterned inspiration. The coordination between the preBötzinger and Bötzinger complexes is important in the transition from inspiration to post-inspiration. Many factors (serotonin, substance $P$, noradrenaline, adenosine) modulate the pre-Bötzinger complex causing an increase or decrease in respiratory rate and amplitude (Doi \& Ramirez, 2010; Ramirez et al., 2012). However, it is mainly due to the surrounding inhibitory neurons near the pre-Bötzinger complex and those that make up the majority of the Bötzinger complex impacting the preBötzinger complex. These populations of GABAergic and glycinergic neurons discharge decrementally during the first phase of expiration (Smith et al., 2013) causing the synchronized and rhythmic firing of Pre-Bötzinger neurons. Finally, the expiratory drive comes from the cVRG, the most caudal group of the respiratory column (Figure 3). The cVRG is connected to the abdominal muscles that produce expiratory activity. The patterned action initiated at the preBötzinger complex and the synchronization followed by the rest of the respiratory column that forms autorhythmic breathing. Projections from the respiratory column, premotor neurons from 
the rVRG included, terminate in nuclei associated with the pump and airway motoneurons such as the phrenic, hypoglossal, and ambiguus nuclei (Bianchi et al., 1995; Doi \& Ramirez, 2008).

\subsection{Discovering the RTN}

Specific areas of the brain responsible for central chemoreception were not explored until the late 1950 's, early 1960 's. During this period there were fundamental discoveries that revolutionized the field of respiration including the first proposed locations of central chemoreceptors and what stimulated them. Loeschcke and colleagues are credited with the initial central chemoreceptor finding when they acidified the fourth ventricle CSF in anesthetized cats and saw an increase in breathing (Loeschcke et al., 1958; Schlaefke et al., 1970). More analysis of the ventral surface of the medulla was conducted by Mitchell's group in the early 1960 's. These experiments were done using acid-soaked pledgets, small cotton wads, which evoked an increase in respiration when applied to certain areas of the medulla. This technique was crude because the acid could have travelled through the arterioles on the ventral surface evoking off-target effects but provided evidence of more localized chemoreceptor(s) (Mitchell et al., 1963a). This general part of the medulla responsive to acid was termed "area M", located just caudal to the trapezoid bodies.

Exploration of central chemoreceptors in the following two decades revealed little until

Smith's group examined retrogradely labelled neurons from the ventral respiratory group (Smith et al., 1989). Some of these neurons were located on/near the ventral surface of the medulla in "area M", leading Smith's group to propose that this cluster of neurons could be involved in chemoreception. They named this group of neurons the retrotrapezoid nucleus (RTN) due to their location behind the trapezoid bodies (Mitchell et al., 1963a; Smith et al., 1989). 
Smith's group and Nattie's group started to use more advanced techniques to examine whether these neurons control breathing. These experiments began as single RTN neuron recordings, iontophoretic application of glutamate analogs and different kinds of microstimulation (Smith et al., 1989). Nattie et al. microinjected kainic acid, a glutamate receptor agonist that strongly activates and then destroys neurons, into the area of the RTN and saw an increase in respiration initially and then attenuation of the hypercapnic ventilatory response (Herndon \& Coyle, 1977; Nattie \& Li, 1990; Nattie et al., 1991). This finding was expanded with experiments using microdialysis probes that could acidify areas of the brain more specifically with $\mathrm{CO}_{2}$ resulting in greater detailed mapping of chemosensitive areas (Li \& Nattie, 1997).

RTN examination at the cellular level began in the early 2000's. Guyenet et al. showed the existence of VGLUT2 mRNA and the lack of serotonin in RTN neurons (Mulkey et al., 2004; Weston et al., 2004). Recently, the Guyenet lab has shown more supporting evidence that the RTN uses glutamate as a transmitter. When RTN neurons were stimulated optogenetically in conscious mice that conditionally lacked VGLUT2 increased breathing was no longer elicited (Holloway et al., 2015). A key advance in the study of the RTN was made when the homeobox domain transcription factor Phox $2 \mathrm{~b}$ was discovered to be expressed in neurons near the facial motor nucleus (where the RTN resides) (Stornetta et al., 2006). This finding led to major advancements in labelling RTN cells histologically, allowing the identification these neurons for in vitro experiments. Lastly, an artificial promoter, PRSx8, was genetically engineered from a dopamine beta-hydroxylase promoter providing greater selectivity for noradrenergic, Phox $2 b$ positive cell populations. PRSx8 proved to be a strong promoter that allowed various transgene 
products to be expressed in the Phox $2 \mathrm{~b}$-positive population (including the RTN) using lenti-viral vectors (Hwang et al., 2001; Lonergan et al., 2005; Abbott et al., 2009a).

\subsection{Disease-relevance}

Respiratory disease is an extremely broad term that encompasses pathological conditions that result in inhibition of gas exchange necessary for an organism to survive. These include but are not limited to central and obstructive sleep apnea, congestive heart failure (CHF), sudden infant death syndrome (SIDS), and congenital central hypoventilation syndrome (CCHS). Problems with neurons regulating respiration have been linked to the diseases listed above but mechanisms remain only partially understood (Amiel et al., 2003, Guyenet et al., 2013, Guyenet \& Mulkey, 2010, Lambert et al., 1995). In many of these diseases malfunction/development issues of the respiratory chemoreceptors are plausible causes of the disease manifestations. Symptoms such as hypoventilation and sleep apneas among others are believed to occur due to central respiratory chemoreceptor loss of sensitivity in detecting salient changes in $\mathrm{PaCO}_{2} / \mathrm{PaO}_{2}$ or neuron malformation (e.g. CCHS) (Amiel et al., 2003; Dauger et al., 2003). As a result there is an inability to maintain healthy eupneic patterned breathing (Sharma et al., 2010).

Breathing problems in humans often occur and/or are aggravated by sleep (Sharma et al., 2010, Mieeczkowski and Ezzie, 2014). Examples of respiratory diseases with symptoms that occur during sleep include central and obstructive sleep apnea, and CCHS (Sharma et al., 2010, Mieeczkowski and Ezzie, 2014). A possible reason for this vulnerability of the respiratory system during sleep could be because the brain is more active during the awake/behaving state and this waking drive to breathe is turned off during sleep, leaving the of regulation of breathing to the brainstem. Thus, when critical areas such as the RTN that maintain eupneic breathing are 
damaged or diseased the problems become intensified during states when the body relies on them most.

A good example of this is observed in CCHS, a rare genetic disease where the Phox $2 \mathrm{~b}$ cells responsible for chemoreception that do not develop correctly, if at all (Dubreuil et al., 2008; Hayasaka K, 2014). Human CCHS patients lose the ability to sense the rise in $\mathrm{PaCO}_{2}$ and drop in pH that normally stimulates breathing (Amiel et al., 2003; Weese-Mayer et al., 2009; Carroll et al., 2014). In mice carrying the most common CCHS mutation, a polyalanine expansion that causes a frameshift mutation (Phox $2 \mathrm{~b} 27 \mathrm{ala} /+$ ), the chemoreflex is almost completely absent and resting ventilation is reduced (Ramanantsoa et al., 2011 and Dubreuil et al., 2009). There is no direct evidence of the loss of RTN neurons in human CCHS patients as of yet but this is most likely due to difficulty obtaining brain samples from patients that had this rare disease.

\subsection{Location and function of the carotid bodies (CBs)}

The carotid bodies (CBs) contribute to resting respiration and the hypoxic ventilatory reflex by sensing $\mathrm{PaO}_{2}$. These small clusters of peripheral chemoreceptors are located bilaterally at the rostral end of the carotid artery where it bifurcates into the internal and external carotid arteries. The CBs receive the highest blood flow per tissue weight of any organ in the body making them ideal for sensing many aspects of blood going to the brain (Barnett et al., 1988). Although the $\mathrm{CBs}$ have been shown to sense changes in temperature, glucose, $\mathrm{PaCO}_{2}$ and certain cytokines, they are most sensitive to the fluctuations in $\mathrm{PaO}_{2}$ (Kumar \& Prabhakar, 2012).

CBs are mainly composed of two types of cells named Type I and Type II cells. The majority of the cells are Type I cells (12,000 cells in the rat and upwards of 60,000 in the cat) (Verna, 1979; McDonald \& Blewett, 1981). Type I cells are the major chemosensitive cell type in 
this organ, becoming excited when exposed to hypoxic environments. Afferent neurons signal through Type I neurons by ACh and ATP, have their cell bodies in the jugular ganglia in the rat and terminate in the commissural or medial subnuclei of the NTS (Finley \& Katz, 1992; Massari et al., 1996). The NTS neurons then influence the respiratory column/RTN. Type II cells in the CBs do not have chemosensitive properties and do not generate action potentials (Duchen et al., 1988; Pardal et al., 2007). They play a supportive role in the CBs analogous to that of astrocytes in the brain. Each Type II cell contacts four to five Type I cells (Ross, 1959). Type II cells make up less than twenty percent of the total cell population and express GFAP, a protein commonly expressed in astrocytes and Schwann cells (Hodges et al., 1975).

The original evidence of the CBs playing a role in sensing their local environment and forwarding information through the glossopharyngeal nerve first came from Fernando de Castro, from the Cajal Institute, where he stated, the CBs were able to "detect qualitative variations in blood, a function which by reflex action will have an influence on the functional activity of other organs"(De Castro, 1926). This theory was further fleshed out in the Nobel Prize winning work done by Jean-Francois Heymans and his son Corneille on vascularly isolated carotid bifurcation experiments in dogs demonstrating the rapid chemosensory reflex of the CBs (Heymans \& Bouckaert, 1930; de Castro, 2009). In all mammals, including humans, carotid body removal produces a significant reduction in resting breathing rate and a loss of the hypoxic ventilatory reflex (Hodges \& Forster, 2012; Mouradian et al., 2012). The respiratory drive and hypoxic ventilatory reflex recover partially or fully over time depending on the species (MartinBody et al., 1986; Timmers et al., 2003; Hodges \& Forster, 2012). There are two potential reasons for the recovery of the hypoxic ventilatory reflex after carotid body removal. Peripheral chemoreceptors that normally do not influence respiration to a great extent such as the aortic 
bodies and ectopic glomus cells may compensate for the lack of $C B$ input by becoming more sensitive and active over time (Martin-Body et al., 1986). Secondly, the brainstem respiratory network has the potential to become hypoxia sensitive in the rat after carotid body denervation (Angelova et al., 2015). This is not the case in the mouse, demonstrated through the work by Chang's group where gene knock-out of Olf-78, which encodes a protein required for sensing $\mathrm{O}_{2}$ in the CBs, results in a permanent absence of the hypoxic ventilatory reflex (Chang et al., 2015). In humans, when the CBs are removed, the hypoxic ventilatory response never recovers (Timmers et al., 2003).

$\mathrm{CBs}$ and the peripheral chemoreflex play essential roles in regulating respiration in many different physiological conditions, including exercise, high altitude, thermogenesis and sleep apnea to name a few. Disrupting gas exchange can result in long term changes in red blood cell count and their $\mathrm{O}_{2}$ loading capacity, angiogenesis and cell metabolism (Semenza, 2012). The CBs respond to changes in $\mathrm{PaO}_{2}$ within seconds (Kumar \& Prabhakar, 2012) and play a large role in the drive to breathe, thus it is critical to study the interaction between these peripheral chemoreceptors and the central chemoreceptors that maintain homeostasis of the body's blood gasses.

\subsection{Optogenetics}

Optogenetics is a biological technique which involves genetically modifying cells to express light-sensitive ion channels or proton pumps on their cell surface to activate or inhibit them. In neuroscience optogenetics primary use is in controlling the activity of individual or populations of neurons in living tissue in order increase or decrease their firing rate. 
A key benefit of optogenetics is the effectiveness of the technique in conscious animal experiments. Studies performed in anesthetized animals or reduced preparations have considerable limitations. The use of lesions or genetic models to explore brain circuits has its own set of issues. For example, in a ventilated anesthetized rat preparation the RPG is silent until the $\mathrm{PaCO}_{2}$ reaches $8-9 \%$ if the vagus nerves are intact. The reason is that anesthetics magnify the lung inflation reflex by potentiating a critical GABAergic relay from the nucleus tractus solitarius (NTS) to the RPG (Takakura et al., 2007). Neuronal lesions are often nonselective and their effect either irreversible or compensated for over time by alternate circuits. The use of saporin conjugated to a substance $\mathrm{P}$ analog (SSP-saporin) to destroy neurons that express NK1R is a fitting illustration of this problem. This drug has been used in the past to target the RTN (Nattie \& Li, 2002; Mulkey et al., 2007). However, the toxin is non-specific for the RTN, NK1R are expressed in multiple neuronal types and probably also include astrocytes causing relatively uncontrolled lesions (Mulkey et al., 2007).

Through the use of channelrhodopsin (ChR2), a blue $(475 \mathrm{~nm})$ light-activated cation channel that depolarizes cells, Abbott et al. reported the effects of stimulating Phox $2 \mathrm{~b}$ neurons in anesthetized and conscious rats (Abbott et al., 2009a; Abbott et al., 2011). The results showed that RTN activation is sufficient to mimic the effects produced by high levels of $\mathrm{CO}_{2}$. The photostimulation of these $\mathrm{CO}_{2}$ activated neurons increases frequency and amplitude of breaths and phrenic nerve discharge, a measure of inspiratory activity (Abbott et al., 2009a; Abbott et al., 2011).

Optogenetic inhibition using a light-activated proton pump isolated from archae has provided insight into the necessity of the RTN on respiration. This proton pump was first discovered in 1983 in the archae, halorubrum (Mukohata et al., 1999). The yellow-green 
(532nm) light-sensitive pump appropriately named archaerhodopsin (Arch) was isolated and successfully expressed on mammalian cellular membranes by Chow et al. in 2010 (Chow et al., 2010). Since then this group has engineered ArchT, a proton pump three times more lightsensitive with excellent expression in mammalian neurons, travelling and expressing throughout the neurons including the axons (Han et al., 2011). ArchT is actived almost instantaneously when exposed to light ( $\sim 8 \mathrm{~ms})$ and once stimulation ends is ready to be activated again only seconds after stimulation ends, making it optimal for behavioral studies. This characteristic renders ArchT superior to halorhodopsin, a chloride pump that hyperpolarizes neurons but remains inactive for tens of seconds post stimulation.

In this dissertation I have tested multiple hypotheses utilizing ArchT. The first hypothesis was that resting breathing is driven to a large extent by RTN neurons and that the breathing stimulation contributed by RTN increases proportionally to the $\mathrm{PaCO}_{2}$. My second goal was to explain why, despite being such a powerful stimulus to the carotid bodies pure hypoxia only produces a modest degree of hyperventilation. My hypothesis was that hypoxia-induced ventilation is limited because the ensuing respiratory alkalosis inhibits RTN neurons. Finally, I planned to test the possibility that breathing is maintained under hyperoxia and returns to control after removing the major peripheral chemoreceptors (the carotid bodies) because of a compensatory increase in the activity of the RTN.

In order to test these hypotheses I examined the breathing effects produced by instant and reversible inhibition of ArchT-transduced RTN neurons in intact unanesthetized rats. The basic assumption was that the degree of breathing inhibition should reflect the mean discharge rate of the ArchT-transduced RTN neurons. ArchT was selectively introduced into RTN neurons of Sprague-Dawley rats using a PRSx8 promoter driven lentiviral vector. RTN inhibition was 
elicited in conscious rats and the effect of breathing frequency and amplitude was monitored under various $\mathrm{O}_{2} / \mathrm{CO}_{2}$ conditions and different states of vigilance including waking, non-REM and REM sleep. We expected to see nearly complete apnea when RTN is inhibited during quiet waking and non-REM sleep due to the dependence on RTN during these states in breathing automaticity. 


\subsection{Figures}

Figure 1.

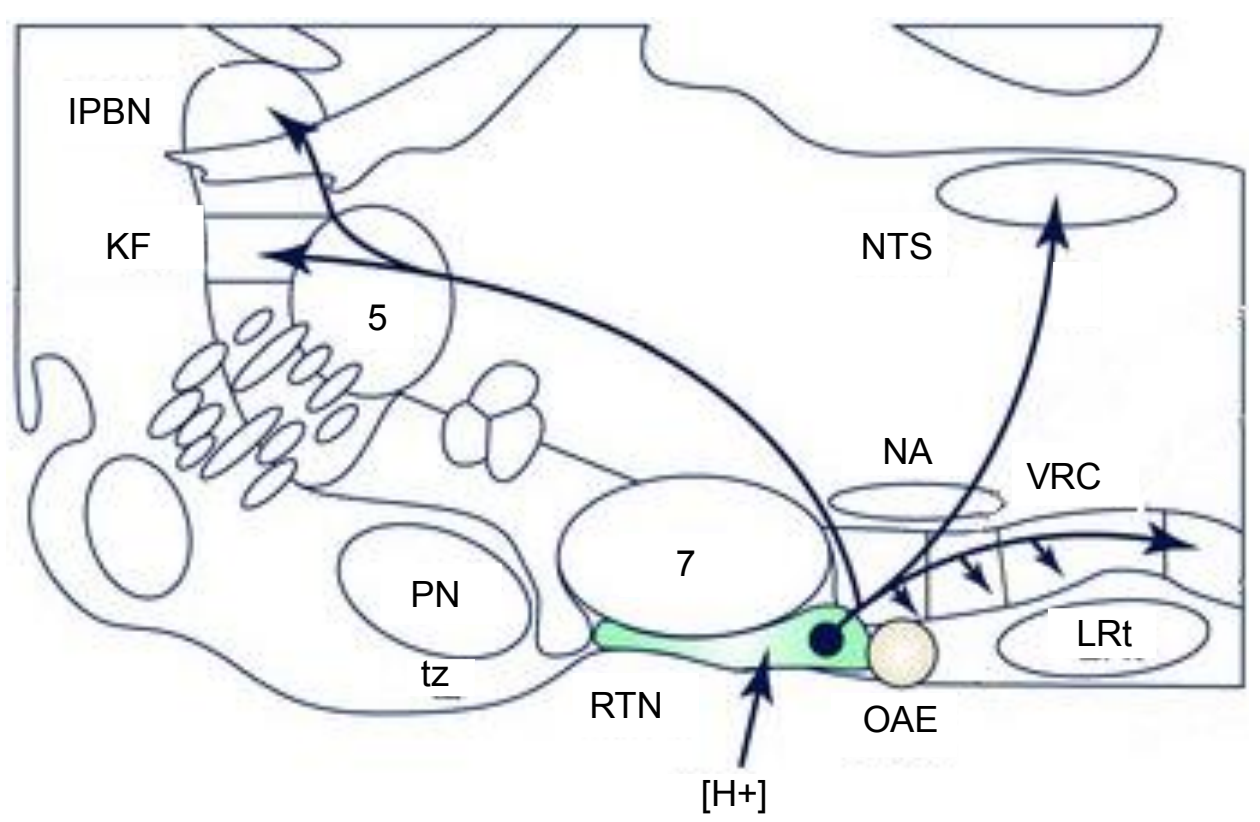


Figure 1: Location and projections of the retrotrapezoid nucleus (RTN). Parasagittal section through the rat's brain ( $1.8 \mathrm{~mm}$ lateral to the midline) illustrating the location of RTN neurons, their axonal projections and the putative function of each projection. Adapted from (Guyenet et al., 2016)

Abbreviations: KF, Kölliker-Fuse nucleus; IPBN, lateral parabrachial nuclei; LRt, lateral reticular nucleus; NA, nucleus ambiguus; NTS, nucleus of the solitary tract; OAE, oscillator for active expiration; PN, pontine nuclei; tz, trapezoid body; VRC, ventral respiratory column 
Figure 2.

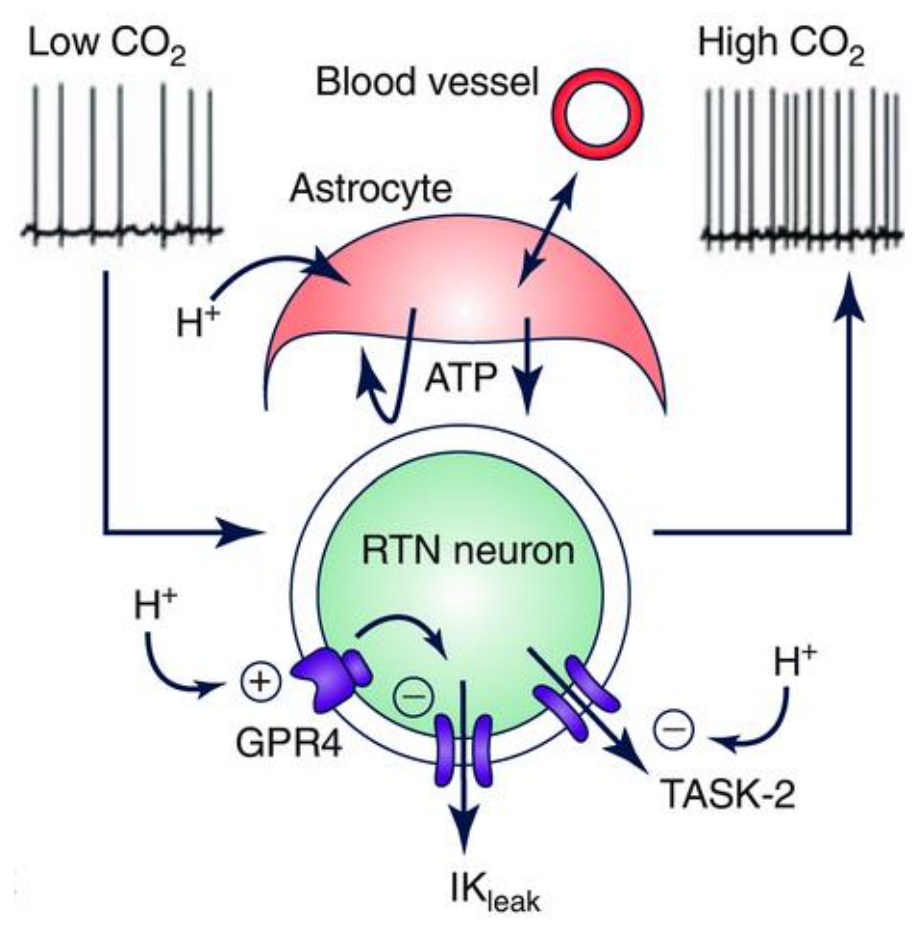


Figure 2: $\mathrm{RTN}$ neurons detect $\mathrm{PaCO}_{2}$ primarily via changes in proton concentration. A recently uncovered mechanism relies on the expression by RTN neurons of a proton-operated G proteincoupled receptor (GPR4) and a proton-sensitive resting potassium conductance (TASK-2) (Wang et al., 2013b; Kumar et al., 2015). Acidification also activates ventral medullary surface astrocytes. In response to this stimulus, their intracellular calcium concentration rises, causing the release of ATP which may directly activate RTN neurons via P2 receptors. Astrocytes may also activate RTN neurons by reducing local blood flow and causing tissue $\mathrm{PaCO}_{2}$ to increase. Figure adapted from (Guyenet et al., 2016) 
Figure 3.

(a)

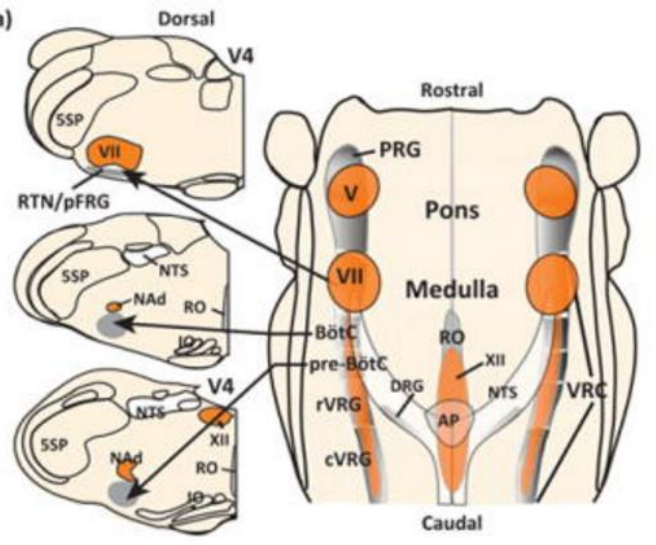

(b)

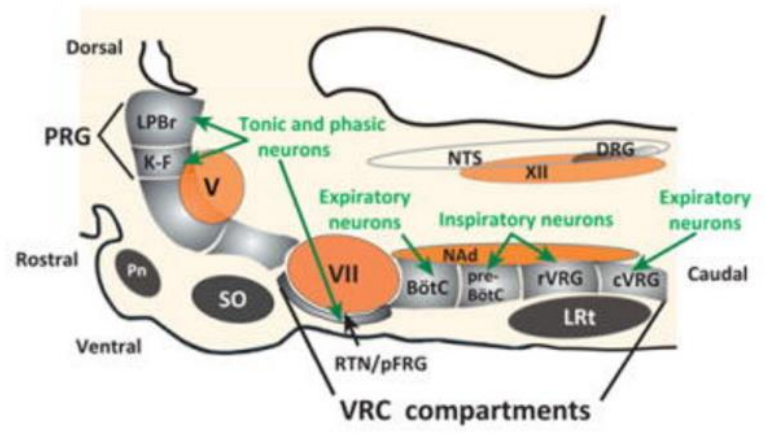


Figure 3: Overview of bilaterally distributed brainstem respiratory compartments of the rat arranged from the rostral pons to the caudal medulla represented neuroanatomically by (a) horizontal (right) and several coronal (left) brainstem sections and (b) a parasagittal section through one side of the brainstem. Predominant locations of inspiratory, expiratory, tonic, and respiratory-modulated (phasic) interneurons are indicated in (b). Other abbreviations: AP, area postrema; LRt, lateral reticular nucleus; NAd, nucleus ambiguus, dorsal division; Pn, ventral pontine nucleus; SO, IO, superior and inferior olivary complexes; SP5, spinal trigeminal nucleus; V, motor nucleus of the trigeminal nerve; V4, fourth ventricle; XII, hypoglossal motor nucleus. Figure obtained from (Smith et al., 2013) 


\section{Chapter 2: Hypoxia silences the retrotrapezoid nucleus respiratory chemoreceptors via alkalosis}

\section{This chapter is a re-print of the Journal of Neuroscience (2015) article (Basting et al., 2015).}

\subsection{Introduction}

The feedback regulation of breathing by blood gases operates via peripheral chemoreceptors, primarily the carotid bodies (CBs), that respond to hypoxia in an aciddependent manner and via central respiratory chemoreceptors (CRCs) that detect brain $\mathrm{PCO}_{2}$ (Takakura et al., 2006; Smith et al., 2010; Guyenet et al., 2010; Huckstepp and Dale, 2011; Kumar and Prabhakar, 2012). Normally, CBs and CRCs work in concert to either stimulate or inhibit breathing because arterial $\mathrm{PO}_{2}$ and brain $\mathrm{PCO}_{2}$ vary in opposite directions when lung ventilation increases or decreases. Under hypoxia, however, the CBs are selectively activated, the resulting hyperventilation causes respiratory alkalosis and CRCs are therefore believed to be inhibited (Gesell et al., 1940). This inhibition could explain why breathing stimulation is inadequate at high altitude and why carbonic anhydrase inhibitors are beneficial in this setting (Pena and Garcia, 2006; Bell and Haouzi, 2010). This plausible interpretation rests on scant neurobiological evidence however because the CRCs have not been definitively identified and their activity has not been directly measured in conscious mammals.

Hyperoxia, commonly used therapeutically, has equally complex effects on breathing. Hyperoxia reduces CB activity which causes an immediate but only transient decrease in breathing (Dejours et al., 1955). At steady-state and depending on the species, hyperoxia causes a slight hypo- or hyperventilation which is attributed to excess CRC activation via cerebral blood flow reduction and other mechanisms (Olson, Jr. et al., 1988; Becker et al., 1996; Ainslie and 
Duffin, 2009; Chowdhuri et al., 2010). The notion that CRCs are activated by hyperoxia also remains to be substantiated.

The retrotrapezoid nucleus (RTN) is a prominent CRC candidate (Smith et al., 1989;

Guyenet et al., 2010; Nattie, 2011). These putatively glutamatergic neurons are strongly activated by hypercapnia in vivo and respond to acidification both directly and via surrounding astrocytes (Mulkey et al., 2004; Gourine et al., 2010; Wang et al., 2013b). RTN innervates the respiratory pattern generator and its selective stimulation activates breathing massively (Abbott et al., 2009a; 2011).

The present experiments represent the first attempt to measure the activity of RTN neurons in conscious rats exposed to various $\mathrm{PO}_{2}$ levels. The objective was to determine whether RTN activity conforms to existing views regarding how CRCs should behave under hypoxic conditions. RTN neurons are located close to the ventral medullary surface and thinly distributed (Stornetta et al., 2006) which precludes unit recording in conscious animals. Fos can be used to gauge RTN activity but this approach lacks the required sensitivity and is restricted to long-lasting perturbations (Sato et al., 1992; Teppema et al., 1997; Fortuna et al., 2009). As a surrogate measure of RTN neuron activity, we monitored the degree of hypoventilation elicited by briefly inhibiting these neurons using Archaerhodopsin (Arch)-based optogenetics (Chow et al., 2010). Because of the slow time-constant of the chemoreflexes, this method allowed us to quantify the instantaneous contribution of the transduced RTN neurons to ventilation at any point in time with minimal interference from homeostatic reflexes.

\subsection{Materials and Methods}

\section{Animals}


Experiments were performed on male Sprague-Dawley rats ( $\mathrm{n}=27 ; 400-550 \mathrm{gm}$, Taconic, USA). All procedures conformed to the NIH Guide for the Care and Use of Laboratory Animals and were approved by the University of Virginia Animal Care and Use Committee. Animals were housed under standard $12 \mathrm{~h}$ light/dark cycle with ad libitum access to food and water.

\section{Viral constructs and virus preparation}

For single-unit experiments in anesthetized rats, we used an adeno-associated viral vector (AAV) that expresses the photoactivatable proton pump Arch-3 fused to eYFP under the control of the pan-neuronal synapsin promoter (AAV-hSyn-eArch3.0-eYFP serotype 2)(Chow et al., 2010). For the experiments on conscious rats, we used a lentiviral vector (LVV) that expresses ArchT3.0, a more light-sensitive version of Arch-3 (Han et al., 2011; Mattis et al., 2012) under the Phox2-responsive promoter PRSx8 (pLenti-PRSX8 eArchT3.0-eYFP)(Hwang et al., 2001). We synthesized the PRSx8-ArchT3.0-eYFP construct by substituting the PRSx8 promoter (kindly provided by M. Raizada, University of Florida) for the CamKIla promoter present in the pLenti CamKIla eArchT 3.0 eYFP construct (courtesy of K. Deisseroth via Addgene). In a few experiments on conscious rats, we also used an already described LVV encoding the photoactivatable cation channel channelrhodopsin-2 (ChR2, H134R) fused to mCherry (PRSx8ChR2-mCherry)(Abbott et al., 2009a; Abbott et al., 2009b). The viral vectors (AAV2 and LVV) were produced by the University of North Carolina virus core. The AAV2 was used at a

concentration of $3.0 \times 10^{12}$ viral particles per ml. Both LVVs were used at a concentration of $3.0 \mathrm{x}$ $10^{8}$ viral particles per $\mathrm{ml}$.

\section{Injections of vectors and animal instrumentation}

The rats were anesthetized with a mixture of ketamine $(75 \mathrm{mg} / \mathrm{kg})$, xylazine $(5 \mathrm{mg} / \mathrm{kg})$ and acepromazine $(1 \mathrm{mg} / \mathrm{kg}$ ) given intraperitoneally (i.p.). Depth of anesthesia was assessed by 
an absence of the corneal and hind-paw withdrawal reflex. Additional anesthetic was administered when necessary ( $25 \%$ of the original dose, i.p. or intramuscularly during surgery). Body temperature was kept close to $37^{\circ} \mathrm{C}$ with a servo-controlled heating pad and a blanket. All surgical procedures were performed under aseptic conditions. The mandibular branch of the facial nerve was exposed on the left side or on both sides as needed. The rat was then placed prone on a stereotaxic apparatus (bite bar set at $-3.0 \mathrm{~mm}$ for flat skull; David Kopf Instruments). A $1.5 \mathrm{~mm}$ diameter hole was drilled into the occipital plate on the left side or both sides caudal to the parieto-occipital suture. The viral vectors were loaded into a $1.2 \mathrm{~mm}$ internal diameter glass pipette broken to a 25 micron tip (external diameter) and ejected by pressure into the RTN 100-200 $\mu \mathrm{m}$ below the facial motor nucleus. The lower edge of the facial motor nucleus was identified using antidromic field potentials evoked in this nucleus by facial nerve stimulation (0.1 ms, 0.2-0.8 mA) (Brown and Guyenet, 1985).

ArchT3.0 LVV was injected bilaterally into three rostrocaudally aligned sites separated by $200 \mu \mathrm{m}$ (total volume $400-600 \mathrm{nl} / \mathrm{side}$ ). The most caudal site was located under the caudal end of the facial motor nucleus. We then implanted optical fibers for light stimulation plus electrodes to record the electroencephalogram (EEG) and neck electromyogram (EMG). For EEG recordings, stainless steel jeweler screws (Plastics One) were implanted extra-durally (0.5 - 1mm anterior, $1 \mathrm{~mm}$ lateral to bregma and $3 \mathrm{~mm}$ posterior, 2-2.5 $\mathrm{mm}$ lateral to bregma above the contralateral hemisphere). One additional screw was implanted for structural support of the head stage and for grounding. Teflon-coated braided stainless steel wire (A-M systems) was stripped at the tip and wrapped around the implanted screws. Two additional wires were stripped at the tips and implanted in the superficial muscles of the neck for EMG recordings of postural activity. All wires were crimped to amphenol pins (A-M systems) and inserted into a 
plastic headstage (Plastics One). The optical fibers (200 $\mu \mathrm{m}$, numerical aperture-0.39; Thorlabs) were fitted with ferrules as described (Sparta et al., 2012) and implanted into the ventrolateral medulla (tips $0.5-0.8 \mathrm{~mm}$ dorsal to the vector injection site). The head-stage and optic fiberferrule assemblies were secured to the skull using a 2-part epoxy (Loctite). Incisions were then closed in two layers (muscle and skin) with absorbable sutures and vet bond adhesive. Rats received post-operative ampicillin (125 mg/kg, i.p.) and ketoprofen (3-5 mg/kg, s.c.) and were monitored daily. Rats recovered for a minimum of four weeks for functional expression of the opsins. A subset of animals were then implanted with radio-telemetry probes (PA-C10, Data Sciences International) to record blood pressure from the descending aorta via the right femoral artery. These rats recovered for yet another week before physiological experiments began.

In six rats, ChR2-expressing LVV was injected unilaterally on the left side (two 100-140 nl injections separated by $200 \mu \mathrm{m})$. These rats were then instrumented in the same way as those that had received injections of ArchT-expressing vector (EMG, EEG, fiber optic) except that a single optical fiber was implanted, on the left side. Four rats received a left side only injection of AAV2-hSyn-eArch3.0-eYFP into RTN (two 100-140 nl injections separated by $200 \mu \mathrm{m}$ ). These rats had no additional surgical procedure. Post-surgical treatment for these two rat groups was as described above.

\section{RTN unit recordings in anesthetized rats}

These experiments were designed to ascertain that Arch-transduced RTN neurons were inhibited by delivering green light in their vicinity through an optical fiber in vivo. These optogenetic experiments were modeled after prior ones in which we tested the light sensitivity of ChR2-transduced RTN neurons (Abbott et al., 2009a). Briefly, 4 rats received injections of AAV-hSyn-eArch3.0-eYFP serotype 2 into the left RTN. Five weeks later the rats were 
anesthetized with isoflurane in pure oxygen ( $5 \%$ for induction, $3 \%$ thereafter), their trachea was cannulated to permit artificial ventilation, and one femoral artery and vein were cannulated to record arterial blood pressure and administer fluids, respectively. RTN units were recorded below the facial motor nucleus as described previously (Guyenet et al., 2005). To deliver green light to the vicinity of Arch-transduced neurons a $200 \mu \mathrm{m}$ optical fiber was inserted at a 20 degree angle from the vertical in the transverse plane and its tip placed an estimated $500 \mu \mathrm{m}$ above the left RTN. The light output at the tip of the fiber optic was adjusted $9 \mathrm{~mW}$ with a light meter (Thorlabs) prior to insertion into the brain. Upon completion of surgical procedures, isoflurane was gradually withdrawn while freshly prepared $\alpha$-chloralose (dissolved in $2 \% \mathrm{Na}$ borate; initial dose: $60 \mathrm{mg} / \mathrm{kg}$; maintenance at $30 \mathrm{mg} / \mathrm{kg} / \mathrm{hr})$ and urethane $(10 \% \mathrm{w} / \mathrm{v}$ in physiological saline; initial dose: $0.7 \mathrm{~g} / \mathrm{kg}$ ) was slowly infused iv and ventilation with $100 \% \mathrm{O}_{2}$ was continued. The adequacy of the anesthesia was assessed by the lack of arterial pressure change and limb withdrawal following a firm paw pinch. Paralysis was induced with vecuronium bromide prior to the recordings (induction dose $0.1 \mathrm{mg} \mathrm{kg}^{-1} \mathrm{iv}$, plus additional $0.05 \mathrm{mg} / \mathrm{kg} / \mathrm{hr}$ infusion for maintenance). End-expiratory $\mathrm{CO}_{2}$ was continuously monitored with a microcapnometer (Columbus Instruments) and maintained between 3.5-4.5\%. Neurons were recorded extracellularly using glass pipettes filled with $2 \mathrm{M} \mathrm{NaCl}(5-10 \mathrm{M} \Omega)$. RTN neurons were characterized as described previously (Guyenet et al., 2005). Their $\mathrm{CO}_{2}$ sensitivity was determined by adding $\mathrm{CO}_{2}$ to the inspired gas in a stepwise manner to increase end-expiratory $\mathrm{PCO}_{2}$ up to $10 \%$ (for further details see (Guyenet et al., 2005)). Phenylephrine (boluses of 10 $\mu \mathrm{g} / \mathrm{kg}$; iv) was administered to raise BP. Briefly, RTN neurons were located 150 to $300 \mu \mathrm{m}$ below the facial motor nucleus, they were activated by hypercapnia (up to $8-12 \mathrm{~Hz}$ at $10 \%$ endexpiratory $\mathrm{CO}_{2}$ ), they were silenced by lowering end-expiratory $\mathrm{CO}_{2}$ below $4 \%$ and they were 
unresponsive to BP elevation. RTN cells discharged tonically or exhibited a mild respiratory modulation at high levels of $\mathrm{FiCO}_{2}$. To test their response to Arch activation, green light was applied continuously in 5-20 s episodes. All analogue data were acquired on a computer via a micro1401 digitizer (Cambridge Electronics (CED)), and were processed off-line using Spike software (CED, for details: (Guyenet et al., 2005)). The spontaneous activity of all recorded units was determined at rest, during the photostimulation period and in recovery.

\section{Physiological experiments in freely-behaving rats}

The rats were tested in a plethysmography chamber (Buxco) modified to allow tethered EEG/EMG recordings and optical stimulation. Before the actual experiments were run the rats were repeatedly habituated to these surroundings which were visually-isolated and with low ambient noise. On the day of the experiment, rats were lightly anesthetized with isoflurane (induction with $5 \%$, maintenance with $2 \%$ in pure oxygen for $<1 \mathrm{~min}$ ) to permit cleaning of hardware and connection to the ferrule and EEG/EMG recording assembly. A $200 \mu$ m-thick multimode optical fiber terminated with a ferrule was mated to the implanted ferrule with a zirconia sleeve. Optical matching gel (Fiber Instrument Sales) was applied at the ferrule junction to reduce light loss. A minimum of $1 \mathrm{hr}$ was allowed for recovery from anesthesia and the emergence of stable sleep/wake patterns. Recordings were made between $10 \mathrm{am}-6 \mathrm{pm}$, over multiple days, with a minimum of three days rest between tests. The ventilatory response to RTN inhibition was assessed using barometric, unrestrained whole-body plethysmography (EMKA Technologies). The plethysmography chamber was continuously flushed with $1.5 \mathrm{~L} / \mathrm{min}$ of $21 \% \mathrm{O}_{2}$ balanced with $\mathrm{N}_{2}$ regulated by computer-driven mass flow controllers for $\mathrm{O}_{2}, \mathrm{~N}_{2}$ and $\mathrm{CO}_{2}$ (Alicat). Temperature and humidity within the plethysmography chamber were kept constant. 
Photoinhibition of Arch-expressing RTN neurons was achieved with a green laser (532 $\mathrm{nm}$, Shanghai Laser and Optics Century) controlled by TTL-pulses from a Grass model S88 stimulator (AstroMed Inc.). The green light was applied bilaterally using a splitter through 200 $\mu m$-thick multimode optical fiber (Thorlabs) in $10 \mathrm{~s}$ episode of continuous illumination. The transmission efficiency of each implantable optical fiber was tested prior to implantation with a light meter (Thorlabs). The light output at the tip of the implanted fibers was $5 \mathrm{~mW}$ prior to implantation into the brain.

\section{Data acquisition and analysis}

Physiological signals were acquired and processed using Spike v7.03 software (Cambridge Electronic Design). EEG and EMG were amplified and band pass filtered (EEG: 0.1$100 \mathrm{~Hz}, \mathrm{x} 1000$. EMG: $100-3000 \mathrm{~Hz}, \mathrm{x} 1000)$ and acquired at a sampling frequency of $1 \mathrm{KHz}$. The signal generated by the differential pressure transducer connected to the plethysmography chamber was amplified, band pass filtered $(x 200,0.1-20 \mathrm{~Hz})$ and acquired at a sampling frequency of $1 \mathrm{KHz}$. The signal from the radio telemetry probe (blood pressure) was acquired at a sampling frequency of $0.2 \mathrm{KHz}$. Mean arterial pressure and heart rate were extracted from pulsatile blood pressure recordings from the descending aorta based on values calibrated prior to implantation of the telemetry probe. Periods of wake or natural sleep were classified on the basis of EEG, EMG activity and the patterns of cardiovascular and breathing activity. During nonrapid eye movement (nREM) sleep, EEG spectra were dominated by delta activity $(0.5-4 \mathrm{~Hz})$, with little or no EMG activity, and a stable breathing pattern, blood pressure and heart rate. Quiet wake was characterized by a reduction in total EEG power with EMG tone and an elevated resting heart rate.

A minimum of 6 photoactivation trials were conducted in each rat in each state (nREM 
and quiet wake), at each level of inspired $\mathrm{O}_{2}(65 \%, 21 \%, 15 \%$ or $12 \%$ poikilocapnic hypoxia), or $\mathrm{CO}_{2}(3 \%)$ or treatment with acetazolamide $(100 \mathrm{mg} / \mathrm{kg}$ in $100 \%$ DMSO, $0.1 \mathrm{ml}$ i.p., Sigma Aldrich) and its control, DMSO. Average breathing values were extracted from all trials not contaminated by body movements, as indicated by EMG activity. Respiratory and cardiovascular parameters $(\mathrm{BP}, \mathrm{HR})$ were averaged during the $10 \mathrm{~s}$ photostimulus. Baseline values were measured during the $20 \mathrm{~s}$ preceding light delivery. Respiratory frequency $\left(f_{R}\right.$, breaths $\left./ \mathrm{min}\right)$ and tidal volume $\left(V_{T}\right.$, area under the curve during the inspiratory period calibrated to waveforms generated by injecting $5 \mathrm{ml}$ of dry air from a syringe during the experiment, expressed in $\mathrm{ml}$ per $100 \mathrm{~g}$ body weight (BW)) were calculated using Spike software v7.3 (CED). These values were used to calculate minute ventilation $\left(V_{E}=f_{R} * V_{T}\right.$, expressed as $\mathrm{ml} / 100 \mathrm{~g}$ body weight/ min $)$.

Following tests for normality, differences within and between groups were determined using either paired Student's t-test, one-way repeated measures (RM) ANOVA, or two-way RM ANOVA with Sidak or Bonferroni multiple comparisons. If data were not normally distributed, Friedman's nonparametric RM ANOVA was conducted with Dunn's post hoc multiple comparisons. All values are expressed as means \pm SEM.

\section{Histology}

Animals were deeply anesthetized with sodium pentobarbital and perfused transcardially with $4 \%$ paraformaldehyde, brains removed and processed as described previously (Abbott et al., 2012). Immunohistochemistry with antibodies against tyrosine hydroxylase (sheep anti-TH, 1:2000, Millipore), eYFP (chicken anti-GFP, 1:1000, AVES labs) or mCherry (rabbit anti-dsRed, 1:500, Clontech \#632496, Clontech Laboratories) and Phox2b (rabbit anti- Phox2b, 1:8000, a gift from J.F. Brunet, Ecole Normale Superieure, Paris, France) 
were performed as previously described (Abbott et al., 2009a). Cell mapping and counting and photography were done using the Neurolucida system (MicroBrightfield, Inc, Colchester, VT) with a Zeiss Axioskop microscope with computer driven stage and Zeiss MRc camera. Cell counts were taken from a 1 in 6 series of sections and only profiles containing a nucleus were counted.

\subsection{Results}

\section{Histology}

The location and phenotype of Arch-transduced neurons (positive for eYFP) were examined by immunohistochemistry in 12 rats (Figure 1 ). Consistent with prior studies using related PRSX8 promoter-containing LVVs (Abbott et al., 2009a), Arch-transduced neurons were limited to a thin layer of Phox2b-ir non-catecholaminergic neurons located underneath the facial motor nucleus (Figure 1A1-A3) and to TH-ir neurons (C1 and A5) occasionally also located under this nucleus but mostly present slightly caudal or medial to it (Figure $1 A 3,4)$. A few eYFP-labeled neurons, presumably cholinergic, were found in 3 cases within the rostral compact portion of nucleus ambiguus and were not included in the count of RTN cells. The TH-negative neurons located under the facial motor nucleus were virtually all Phox2b-positive and classified as RTN neurons (Abbott et al., 2009a). The rostrocaudal distribution of Arch-transduced RTN and catecholaminergic (CA) neurons is shown in Figure 1B (12 rats). The total number of Archtransduced RTN neurons counted in a one in six series of coronal sections was $85.8 \pm 11.3$ neurons. The number of transduced CA neurons was $81.2 \pm 9$. Figure $1 \mathrm{C}$ shows where the tips of the optical fibers were located in 10 of these rats (Figure1B,C). Two of the 12 rats had a correctly placed fiber optic on one side only and are not represented. 
The distribution of ChR2-transduced neurons was very similar albeit unilateral by design. On average, $67 \pm 11$ neurons (one-in-six section counts) expressed eYFP, of which $33 \pm 8$ also expressed TH (47\%). The optical fiber tips were located $0.5-1 \mathrm{~mm}$ dorsal to the ventral medullary surface at levels 9.7-11.5 caudal to bregma similar to the placement of the optical fibers in the Arch cases shown in Figure 1.

\section{Photoactivation of the proton pump silences RTN neurons in anesthetized rats}

Archaerhodopsin is a light-activated pump that translocates protons from the cytoplasm to the extracellular space, thereby hyperpolarizing and silencing neurons (Chow et al., 2010). Because RTN neurons are activated by acidification (Mulkey et al., 2004; Wang et al., 2013a; 2013b), we wanted to determine that Arch activation does silence RTN neurons, i.e. that the protons translocated by the pump do not stimulate these chemosensitive cells. We identified 9 RTN neurons in 4 rats in which RTN neurons were transduced with Arch. Six of those were silenced by applying green light for 2-10s (Figure 2A1). The rest were unaffected. Inhibition was immediate, instantly reversible and complete including during the time the neurons were maximally activated by $10 \% \mathrm{CO}_{2}$ (Figure $2 \mathrm{~A} 1, \mathrm{~A} 2$ ). The inhibited neurons had the characteristic properties of previously identified Phox $2 \mathrm{~b}$-positive RTN chemoreceptors, i.e. they were silent below $3.5-4.5 \%$ end-expiratory $\mathrm{CO}_{2}$, robustly activated by hypercapnia in a concentrationdependent manner and respiratory-modulated at high levels of $\mathrm{CO}_{2}$ only (Figure $2 \mathrm{~A} 2, \mathrm{~B}$ ). Thus, despite their intrinsic sensitivity to acid, RTN neurons are inhibited by Arch like other previously tested neurons. Given the uncertainty regarding whether intra- or extra-cellular acidification is responsible for the acid-induced depolarization of RTN neurons, several explanations are plausible. First, the Arch-dependent outward current delivers too few protons to acidify the 
extracellular space. Second, the pump generates an outward current that is far greater than the maximal inward current caused by extracellular acidification in RTN neurons (typically 20-30 pA in slices) (Mulkey et al., 2004; Wang et al., 2013a; Wang et al., 2013b). Third, if the potassium conductance responsible for the acid-sensitivity of RTN neurons is closed by intracellular rather than extracellular acidification, the intracellular alkalization caused by Arch could actually contribute to inhibiting RTN neurons.

\section{Arch-triggered hypoventilation is attenuated by hypoxia}

These physiological experiments were designed to test several predicted consequences of the theory that RTN neurons are CRCs namely i) RTN neuron inhibition should produce hypoventilation at rest, ii) RTN neurons should be silenced by hypoxia, iii) during hypoxia RTN neurons should be reactivated by restoring blood acidity to control (normoxic) levels and, iv) RTN neurons should be activated by hyperoxia. Collectively these four criteria form a reasonably stringent test to identify CRCs in vivo. The first requirement distinguishes between neurons that drive breathing at rest and neurons that do not. The second one distinguishes between putative CRCs and neurons that merely contribute to respiratory rhythm and pattern generation. Contrary to CRCs, the latter neurons should be activated by hypoxia because this stimulus activates the breathing rate and amplitude. The third criterion verifies the expectation that hypoxia inhibits RTN neurons via alkalization. Because hyperoxia decreases breathing in rats (Olson, Jr. et al., 1988), the fourth criterion also distinguishes potential CRCs (activation by hyperoxia) from neurons that contribute to respiratory rhythm and pattern generation because the activity of the latter should be reduced in a manner commensurate with the reduction in breathing intensity. Criteria 1, 2 and 4 are addressed in this section; the third criterion is examined later on. 
Blood gases were measured while the rats were quiescent and fully habituated to their surroundings to minimize the confounding effect of stress and physical activity on ventilation. Blood gases during normoxia, hypoxia and hyperoxia conformed to expectations for rats (Olson, Jr. et al., 1988) (Table). Hyperoxia caused a slight respiratory acidosis and hypoxia caused frank respiratory alkalosis which was more pronounced under $12 \%$ than $15 \% \mathrm{O}_{2}$. Archaerhodopsin was photoactivated exclusively during periods of eupneic breathing i.e. when the rats were quietly awake or in non-REM sleep and breathing was regular. Periods of REM sleep or episodes during which the rats were actively behaving (grooming, sniffing, ambulating) were deliberately excluded from testing.

Hyperoxia reduced resting ventilation slightly whereas hypoxia produced the expected oxygen concentration-dependent stimulation of breathing (Figure 3A,B). Arch activation consistently reduced breathing rate and amplitude during normoxia (Figure $3 \mathrm{~A}, \mathrm{~B}$ ). Archinduced hypoventilation was increased during hyperoxia, attenuated under $15 \%$ hypoxia and dramatically reduced at $12 \% \mathrm{O}_{2}$ (Figure $3 \mathrm{~A}-\mathrm{C}$ ). Arch-induced hypoventilation was maximal during the early part of the photostimulus and tended to return towards the pre-stimulation level thereafter. The tendency for breathing to increase during the latter part of the photostimulus period can be better appreciated by examining event-triggered averaged responses (Figure 4). These graphs show that the hypoventilation was better sustained throughout the light stimulus during hyperoxia than during hypoxia. This in turn suggests that, during normoxia and especially so during hypoxia, the hypoventilation elicited by the photostimulus was rapidly attenuated by an increase in CB activity rather than by an increase in CRC activity. This interpretation is based on the fact that the peripheral chemoreflex has a much shorter time constant than the central chemoreflex (Smith et al., 2006). The relaxation of the 
hypoventilation during the latter part of the photostimulus indicates that we slightly underestimated the instantaneous contribution of RTN neurons to resting ventilation by averaging the breathing frequency and tidal volume throughout the $10 \mathrm{~s}$ stimulus period.

Arterial pressure (BP) was recorded by telemetry in 4 rats. Resting BP was unaffected by the level of inhaled oxygen (Figure 5A) but Arch photoactivation (10 s) produced a small hypotension that was increased under hypoxia (Figure 5B). The rostral C1 cells innervate and drive vasomotor sympathetic preganglionic neurons. Their activity is increased by hypoxia and helps maintain BP during hypoxia (Sun and Reis, 1994; Hirooka et al., 1997; Madden and Sved, 2003). Hypoxia-dependent hypotension was therefore an expected consequence of the fact that a subset of $\mathrm{C} 1$ neurons was transduced with Arch. The hypotension was very small however suggesting that few $\mathrm{C} 1$ cells were inhibited or that the baroreflex strongly buffered the response by activating the nontransduced $\mathrm{C} 1$ neurons.

The hypoventilation elicited by activating Arch was correlated with the number of transduced RTN neurons (Pearson $r=0.64$, one-tailed $p=0.027$ for $\mathrm{f}_{\mathrm{R}} ; r=0.66, p=0.019$ for $\mathrm{V}_{\mathrm{T}} ; r$ $=0.59, p=0.035$ for $V_{E}$ ) and exhibited only a weak correlation with the number of transduced CA cells (Pearson $r=0.52$, one-tailed $p=0.064$ for $\mathrm{f}_{\mathrm{R}} ; r=0.55, p=0.049$ for $\mathrm{V}_{\mathrm{T}} ; r=0.60, p=0.033$ for $V_{E}$ ) (Figure $\left.6 A, B\right)$. However when we considered only the transduced neurons located within $500 \mu \mathrm{m}$ of the coronal plane of the optical fibers (10 rats; Figure $6 \mathrm{C}$ ), the correlation between hypoventilation and transduced RTN neurons became much tighter $\left(r=0.74 p=0.008\right.$ for $\mathrm{f}_{\mathrm{R}} ; r=$ $0.86, p=0.0008$ for $\mathrm{V}_{\mathrm{T}} ; r=0.86, p=0.0007$ for $\mathrm{V}_{\mathrm{E}}$ ) whereas there was even less correlation with the number of transduced CA cells located in the same domain $\left(r=0.46, p=0.093\right.$ for $\mathrm{f}_{\mathrm{R}} ; r=0.55$, $p=0.051$ for $\mathrm{V}_{\mathrm{T}} ; r=0.62, p=0.027$ for $\mathrm{V}_{\mathrm{E}}$ ) (Figure 6D). Linear regression analysis further 
revealed that the relationship between number of transduced RTN cells and the respiratory effects produced during Arch photoinhibition was more robust than that between numbers of CA neurons transduced particularly when distance from the fiber optic tip was taken into consideration (Figure 6). Thus, the hypoventilation elicited by the light was likely caused by inhibition of Arch-transduced RTN neurons as opposed to catecholaminergic neurons, and could not have been an artifact of the light itself. The results also suggest that the light may not have been able to reach the entire population of transduced RTN neurons. In this cohort of 10 rats the number of Arch- transduced RTN neurons located within $500 \mu \mathrm{m}$ of the tip of the optical fibers varied from a low of 120 to a high of 480 (the number of counted neurons in a one-in-six series of sections multiplied by 6 ) and the total number of transduced RTN neurons was between 240 and 980 . Since the rat has approximately 2000 RTN neurons, we estimate that the maximum percentage of RTN neurons that could have been directly photoinhibited could not have exceeded $50 \%$ and yet $V_{E}$ could be reduced up to $80 \%$.

\section{Does arousal account for the loss of Arch-triggered hypoventilation during hypoxia?}

Hypoxia causes arousal (Pappenheimer, 1977; Phillipson et al., 1978) and the contribution of RTN neurons to breathing has been previously described as state-dependent (Li et al., 1999). In order to test whether the loss of Arch-triggered hypoventilation during hypoxia was caused by arousal, we compared the responses elicited under the following three conditions: in normoxia when the rats were in NREM sleep, in normoxia while the animals were quietly awake and in $12 \%$ hypoxia. The state of vigilance was determined by video recording of the animal's immobility and posture and by examining the delta power $(0.5-4 \mathrm{~Hz})$ of the EEG. The hypoventilation elicited during NREM sleep was about the same as when the rats were 
quietly awake (Figure 7). The hypoventilation observed during hypoxia was still significantly smaller than when the rats were awake under normoxia (Figure 7B). FFT analysis of the EEG showed that the delta power $(0.5-4 \mathrm{~Hz})$ was the same during quiet waking and hypoxia and that the delta power under both conditions was significantly less than during NREM sleep (Figure 7C). According to these results, arousal does not account for the loss of Arch-triggered hypoventilation during hypoxia. More importantly, and in contradiction with prior results (Li et al., 1999), our results show that RTN neurons drive eupneic breathing at least equally during NREM and quiet waking in rats.

\section{Is the stimulatory effect of RTN on breathing attenuated by hypoxia?}

A possible reason why Arch-triggered hypoventilation is attenuated in hypoxia could be that the respiratory network cannot respond any longer to RTN neuronal input under this condition. In order to address this question we stimulated RTN neurons optogenetically using a previously described ChR2-expressing lentiviral vector (PRSx8-ChR2-eYFP-LVV) (Abbott et al., 2009a) with the same cell specificity as the Arch vector. The increase in breathing frequency elicited by photostimulation of the ChR2-transduced neurons was only slightly reduced by hypoxia ( $25 \%$ decrease at $12 \% \mathrm{FiO}_{2}$, Figure $8 \mathrm{~A}, \mathrm{~B}$ ). The increase in tidal volume elicited by RTN stimulation was reduced to a much greater degree by hypoxia ( $50 \%$ on average at $12 \% \mathrm{FiO}_{2}$, Figure $8 \mathrm{~A}, \mathrm{C})$. Thus, the effect of RTN neurons on respiratory frequency was close to normal in hypoxia but the relationship between RTN activity and inspiratory amplitude was considerably reduced. 


\section{The loss of Arch-triggered hypoventilation during hypoxia is reversed by re-acidifying the blood.}

These experiments were designed to test the third criterion: if RTN is silenced by hypoxia as a result of respiratory alkalosis, RTN should be reactivated by reacidifying the blood. Reacidification was achieved by exposing the rats to $12 \% \mathrm{O}_{2}$ supplemented with $3 \% \mathrm{CO}_{2}$ or by administering the carbonic anhydrase inhibitor acetazolamide, a drug used clinically to stimulate breathing at altitude.

The addition of $3 \% \mathrm{CO}_{2}$ to the $12 \%$ hypoxic breathing mixture restored blood $\mathrm{pH}$ and $\mathrm{PCO}_{2}$ towards the values observed under normoxia although a slight respiratory alkalosis still persisted ( $\mathrm{pH} 7.48$ vs. 7.44; $\mathrm{PCO}_{2} 34.5$ vs. 41.8; Table). The addition of $\mathrm{CO}_{2}$ also restored the Arch-induced hypoventilation towards the level observed in normoxia (Figure 9A). However, contrary to expectations, hypercapnia restored only the bradypnea elicited by RTN inhibition (Figure 9A, B1). Hypercapnia had no effect on the $V_{T}$ reduction elicited by RTN inhibition under any of the oxygen concentrations tested (Figure 9A,B2).

DMSO is the solvent for acetazolamide, thus we compared the ventilatory responses to Arch activation following DMSO administration alone to the responses following acetazolamide administration. DMSO and acetazolamide were administered to the same 6 rats in random order at an interval of three days. Acetazolamide reduced plasma bicarbonate as expected but had no effect on $\mathrm{PCO}_{2}$ regardless of the level of inspired oxygen (Table). Arterial pH was acidified at all levels of inspired oxygen and remained remarkably constant (mean $0.06 \mathrm{pH}$ change between hyperoxia and $12 \% \mathrm{O}_{2}$ compared to $0.28 \mathrm{pH}$ change in DMSO-treated controls, Table). Archinduced hypoventilation was strongly dependent on $\mathrm{FiO}_{2}$ (inhaled oxygen level) in the control 
group (DMSO, Figure 10). Acetazolamide increased resting ventilation under all oxygen conditions (not shown). After administration of this drug, Arch-induced hypoventilation persisted under all oxygen conditions tested (Figure 10A,B). However, as in the $\mathrm{CO}_{2}$ supplementation experiments, acetazolamide restored only the bradypnea evoked by inhibiting the RTN (Figure 10A4,B1). In contrast, acetazolamide had no effect on the $\mathrm{V}_{\mathrm{T}}$ reduction elicited by RTN inhibition under any of the oxygen concentrations tested (Figure 10A4,B2).

In summary, during hypoxia, blood acidification restored the ability of RTN inhibition to reduce the respiratory frequency but this procedure had no detectable influence on the concomitant reduction of breathing amplitude.

\subsection{Discussion}

We found that RTN drives breathing $\left(f_{R}\right.$ and $V_{T}$ ) equally during non-REM sleep and quiet waking. The frequency reduction caused by RTN inhibition is eliminated by hypoxia and reinstated by blood acidification, therefore attributable to the inhibition of neurons with CRC characteristics. The $\mathrm{V}_{\mathrm{T}}$ decrease is reduced by hypoxia but unaffected by blood reacidification and may be mediated by $\mathrm{CO}_{2}$-insensitive RTN neurons.

Interpretative limitations

The C1 cells are strongly activated by hypoxia, primarily regulate blood pressure but also increase ventilation (Abbott et al., 2013; Guyenet et al., 2013). Yet, C1 cell inhibition is unlikely to have caused the bradypnea elicited by Arch stimulation because this response, like the hypotension, should have been increased, not decreased by hypoxia and no correlation was 
found between the hypoventilation and the number of Arch-transduced CA neurons located close to the fiber optic.

We gauged the activity of RTN neurons by how much breathing inhibition resulted from photoactivating Arch. This interpretation assumes that hypoxia did not attenuate the transfer function between RTN neuron activity and $f_{R}$ or $V_{T}$ and that the transduced neurons were silenced by the proton pump in all circumstances (rest, hypoxia, hypercapnia). ChR2-mediated stimulation of RTN neurons caused a similar tachypnea during hypoxia, validating the first assumption with respect to $f_{R}$. However, the $V_{T}$ increase elicited by RTN stimulation was greatly reduced by hypoxia. Respiratory muscle fatigue or a depressant effect of hypoxia on the respiratory network must therefore be considered when interpreting the effects of RTN inhibition on $\mathrm{V}_{\mathrm{T}}$. We verified the second assumption albeit only in anesthetized animals by showing that RTN neurons were silenced regardless of their resting discharge rate.

\section{RTN regulates eupneic breathing}

In normoxia, RTN inhibition reduced breathing equally during non-REM sleep and quiet waking. These results differ from $\mathrm{Li}$ et al ( 1999) who, using $\mathrm{CO}_{2}$ microdialysis experiments, proposed that RTN drives breathing only in awake rats. Our results also differ from Marina et al. (2010) who concluded that inhibiting RTN reduces the hypercapnic ventilatory reflex but has no effect on resting ventilation. These authors used the allatostatin method (Tan et al., 2006) and transduced RTN neurons with a PRXS8-based LVV. Accordingly, we were targeting the same neuronal population. We explain this discrepancy as follows. The Arch method measures the instantaneous respiratory drive contributed by the transduced RTN neurons with minimal interference from the chemoreflex whereas the allatostatin method measures the effect of RTN 
inhibition at steady-state following chemoreflex compensation. As shown here, the hypoventilation elicited by inhibiting a fraction of RTN neurons is rapidly attenuated, presumably by the gradual recruitment of the CBs or nontransduced RTN neurons.

Sleep apnea, the defining feature of the congenital central hypoventilation syndrome occurs predominantly during non-REM sleep and is attributed to Phox $2 \mathrm{~b}$ mutations that abort RTN development (Pattyn et al., 1999; Amiel et al., 2003; Amiel et al., 2009; Weese-Mayer et al., 2010; Goridis and Brunet, 2010; Ramanantsoa et al., 2011). This theory is strongly supported by our finding that RTN sustains breathing during non-REM sleep.

Breathing frequency is regulated by $\mathrm{CO}_{2}$-sensitive $\mathrm{RTN}$ neurons

Hypoxia in conscious mammals is suspected to silence CRCs via respiratory alkalosis (Gesell et al., 1940). As shown here, a subset of RTN neurons have this predicted but heretofore never demonstrated characteristic. Our interpretation, summarized in Figure 11, is based on the fact that RTN photinhibition was not effective at inhibiting $f_{R}$ during hypoxia unless blood alkalization was prevented by $\mathrm{CO}_{2}$ supplementation or acetazolamide administration (Pena and Garcia, 2006; Bell and Haouzi, 2010). We dismissed the possibility that RTN neurons remain active during hypoxia while their effect on breathing is gated out downstream (see "experimental limitations"). We also showed that the difference between hypoxia and normoxia could not have reflected a difference in the state of vigilance (Li et al., 1999).

By acidifying the plasma, hypercapnia and acetazolamide also presumably potentiated the CB response to hypoxia (Figure 11) (Kumar and Prabhakar, 2012; Prabhakar, 2013). RTN reactivation by $\mathrm{CO}_{2}$ or acetazolamide could therefore have partly resulted from an increased polysynaptic excitatory input from the CBs (Takakura et al., 2006). 
Is the tidal volume regulated by $\mathrm{pH}$-insensitive RTN neurons?

Inhibiting Arch-transduced neurons reduced $V_{\mathrm{T}}$. This effect was also reduced by hypoxia but this effect of hypoxia was unaffected by hypercapnia or acetazolamide. The $V_{T}$ reduction elicited during normoxia may therefore have resulted from the inhibition of $\mathrm{CO}_{2}$-insensitive RTN neurons and the effect of hypoxia from inhibitory feedback from the respiratory pattern generator (RPG) and lung afferents to RTN rather than alkalosis (Figure 11) (Guyenet et al., 2005; Moreira et al., 2007; Takakura et al., 2007). Two populations of Phox $2 b^{+}$RTN neurons with differing sensitivity to acid have indeed been described in vitro (Lazarenko et al., 2009) and the existence of $\mathrm{CO}_{2}$-insensitive RTN neurons could explain why only a subset of these neurons express Fos after hypercapnia (Fortuna et al., 2009).

However, as indicated by the ChR2 experiments, the transfer function between RTN neuronal activity and $V_{T}$ was attenuated by hypoxia. This attenuation, likely caused by muscle fatigue or CNS depression, could partly explain why hypoxia reduced the $\mathrm{V}_{\mathrm{T}}$ inhibition elicited by silencing RTN. Blood acidification $\left(\mathrm{CO}_{2}\right.$, acetazolamide) produces further muscle fatigue (Juan et al., 1984). The additional reduction in transfer function between RTN and respiratory muscle contractions could have canceled out the stimulatory effect of CNS acidification on chemosensitive RTN neurons.

Carotid body stimulation activates $f_{R}$ predominantly via pathways that bypass the RTN

A long-standing theory posits that central and peripheral chemoreceptor pathways converge on a common set of CNS neurons that ultimately regulate the RPG (Gesell et al., 1940; Loeschcke, 1982). The RTN could be one such nodal point for the following reasons. Under isocapnic conditions, CB stimulation activates RTN neurons (Takakura et al., 2006). Muscimol 
injection into RTN decreases both the peripheral and central chemoreflexes in awake rats (Takakura et al., 2013). Finally, RTN neurons receive excitatory input from CRC candidates such as serotonergic, orexinergic neurons (Mulkey et al., 2007; Li and Nattie, 2010; Lazarenko et al., 2011; Nattie, 2011). However, according to the present results, RTN cannot be an obligatory relay for the peripheral chemoreflex because during hypoxia, a condition where the CBs are vigorously activated and breathing is stimulated, RTN inhibition had little effect. Therefore, at least under poikilocapnic hypoxia, the CBs must activate breathing predominantly via pathways that bypass RTN (Figure11). This interpretation agrees with neuroanatomical evidence. CB second-order neurons target multiple levels of the ventral respiratory column plus regions of the dorsolateral pons implicated in respiratory control (Koshiya and Guyenet, 1996; Song et al., 2011). These regions are also targeted by RTN (Bochorishvili et al., 2012), therefore integration between central and peripheral chemoreflexes likely occurs at multiple sites. Some integration undoubtedly occurs at the RTN (Takakura et al., 2006) but at least under hypoxia, the excitatory input that these neurons receive from the CBs seems insufficient to activate them, presumably because of the dominant inhibitory effect of alkalosis.

Hyperoxia increases the contribution of RTN neurons to breathing

In Sprague-Dawley rats, acute hyperoxia ( $\sim 30 \mathrm{~min})$ increases $\mathrm{PaCO}_{2}$ and produces mild hypoventilation (present results; (Olson, Jr. et al., 1988)). These effects are directionally similar, albeit smaller, than those elicited by CB denervation and are consistent with prior evidence that the $\mathrm{CBs}$ are silenced by hyperoxia and contribute significantly to resting ventilation even in normoxia (Olson, Jr. et al., 1988; Forster et al., 2008; Smith et al., 2010). 
In conscious rats, silencing RTN produced a greater inhibition of breathing in hyperoxia than during normoxia. Thus, the relative contribution of RTN neurons to breathing is greatly increased when the $\mathrm{CBs}$ are inactive. Unit recordings would be required to ascertain that the discharge rate of RTN neurons was actually increased by hyperoxia but this is probable for several reasons. In rats, hyperoxia causes a mild respiratory acidosis (present results; (Olson, Jr. et al., 1988)). Hyperoxia also causes additional brain acidification via the Haldane effect and brain vasoconstriction (Becker et al., 1996; Ainslie and Duffin, 2009; Chowdhuri et al., 2010). In larger species including man, the latter effects more than compensate for the withdrawal of $C B$ afferent activity because breathing is slightly activated by hyperoxia at steady-state and a mild respiratory alkalosis is observed (Becker et al., 1996).

In short, hyperoxia increases the relative contribution of RTN to eupneic breathing by reducing $C B$ activation and, possibly, via brain acidification (Figure11).

\section{Pathophysiological implications}

Sleep apnea and/or periodic breathing are associated with hypoxia and several pathologies in which $\mathrm{CBs}$ are hyperactive, namely congestive heart failure (CHF) and hypertension (McBryde et al., 2013; Schultz et al., 2013; Dempsey et al., 2014). Given the present results, CB hyperactivity should reduce RTN neuron activity if it is unaccompanied by acidosis or hypercapnia. According to established reasoning, this should reduce the $\mathrm{CO}_{2}$ reserve and promote apneas or periodic breathing (Ainslie and Duffin, 2009; Cui et al., 2012; Javaheri and Dempsey, 2013). Our results also account for the therapeutic benefits of mild hypercapnia and acetazolamide in hypoxia and CHF (Sharma et al., 2011). 
Table: blood gases in conscious rats

\begin{tabular}{|c|c|c|c|c|c|}
\hline Variable & $\mathbf{n}$ & $\mathrm{pH}$ & $\mathrm{paCO}_{2}$ & $\mathrm{paO}_{2}$ & $\mathrm{HCO}_{3}$ \\
\hline$@ 65 \% 0_{2}$ & 6 & $7.4 \pm 0.01$ & $49.2 \pm 2.3$ & $270.9 \pm 8.2$ & $30.4 \pm 1.2$ \\
\hline$@ 21 \% 0_{2}$ & 6 & $7.44 \pm 0.01$ & $41.8 \pm 1.9$ & $78.3 \pm 3$ & $29 \pm 1.7$ \\
\hline$@ 15 \% \mathrm{O}_{2}$ & 6 & $\begin{array}{c}7.51 \pm 0.01 \\
(t)\end{array}$ & $32.6 \pm 0.9$ & $51.5 \pm 2.6$ & $\begin{array}{c}26.1 \pm 0.8 \\
(\dagger)\end{array}$ \\
\hline$@ 12 \% 0_{2}$ & 6 & $\begin{array}{c}7.59 \pm 0.01 \\
(*,+++)\end{array}$ & $\begin{array}{l}25 \pm 0.6 \\
(*,+t+)\end{array}$ & $43 \pm 2.4$ & $\begin{array}{c}23.9 \pm 0.9 \\
(*,+t+)\end{array}$ \\
\hline $\begin{array}{c}@ 12 \% \mathrm{O}_{2}+ \\
3 \% \mathrm{CO}_{2}\end{array}$ & 5 & $7.48 \pm 0.02$ & $34.5 \pm 0.9$ & $56.6 \pm 3.3$ & $25.7 \pm 0.3$ \\
\hline ACTZ @65\% O & 4 & $7.36 \pm 0.01$ & $40.6 \pm 2.0$ & $286.8 \pm 6.8$ & $22.5 \pm 0.5$ \\
\hline ACTZ @21\% O & 4 & $7.38 \pm 0.01$ & $40.3 \pm 1.4$ & $100 \pm 2.2$ & $24 \pm 0.6$ \\
\hline ACTZ @15\% O & 4 & $7.4 \pm 0.01$ & $32.7 \pm 0.6$ & $63.8 \pm 1.5$ & $20.2 \pm 0.7$ \\
\hline ACTZ @12\% O & 4 & $\begin{array}{c}7.43 \pm 0.01 \\
(†)\end{array}$ & $\begin{array}{c}28 \pm 0.8 \\
(\dagger)\end{array}$ & $51.5 \pm 2.0$ & $\begin{array}{c}18.5 \pm 0.8 \\
(*)\end{array}$ \\
\hline
\end{tabular}

Blood gases were measured in quietly resting rats habituated to the plethysmography chamber during exposure to various breathing mixtures. ACTZ: acetazolamide. Statistics: nonparametric Friedman's one-way RM ANOVA across four levels of inspired $\mathrm{O}_{2}(\mathrm{n}=6)$ for $\mathrm{pH}, \mathrm{paCO}_{2}, \mathrm{paO}_{2}$ and $\mathrm{HCO}_{3}$ and after acetazolamide treatment measuring the same dependent variables $(n=4)$.

Friedman statistic ( $\mathrm{F}$ ) for $\mathrm{O}_{2}$ levels and $\mathrm{pH}=18.0, p<0.0001$; for $\mathrm{paCO}_{2}: \mathrm{F}=17.0, p<0.0001$; for $\mathrm{paO}_{2}: \mathrm{F}=18.0, p<0.0001$; for $\mathrm{HCO}_{3}: \mathrm{F}=16.0, p<0.0001$. Freidman statistic for acetazolamide and $\mathrm{pH}=11.1, p<0.0009$; for $\mathrm{paCO}_{2}: \mathrm{F}=11.1, p<0.0009$; for $\mathrm{paO}_{2}: \mathrm{F}=12.0, p<0.0001$; for $\mathrm{HCO}_{3}: \mathrm{F}=11.1, p<0.0009$. Dunn's multiple comparisons showed $\mathrm{p}$ values $<0.05$ between hypoxia $\left(12 \% \mathrm{O}_{2}\right)$ and normoxia $\left(21 \% \mathrm{O}_{2}\right)$ for $\mathrm{pH}$, paCO , and $\mathrm{HCO}_{3}$, or hyperoxia $\left(65 \% \mathrm{O}_{2}\right)$ as 
indicated: + compares $65 \%$ vs 15 or $12 \% \mathrm{O}_{2},{ }^{*}$ compares $21 \%$ vs 15 or $12 \% \mathrm{O}_{2}$. One symbol $p<$ 0.05 , two symbols $p<0.01$. three symbols $p<0.005$. 


\subsection{Figures}

Figure 1.
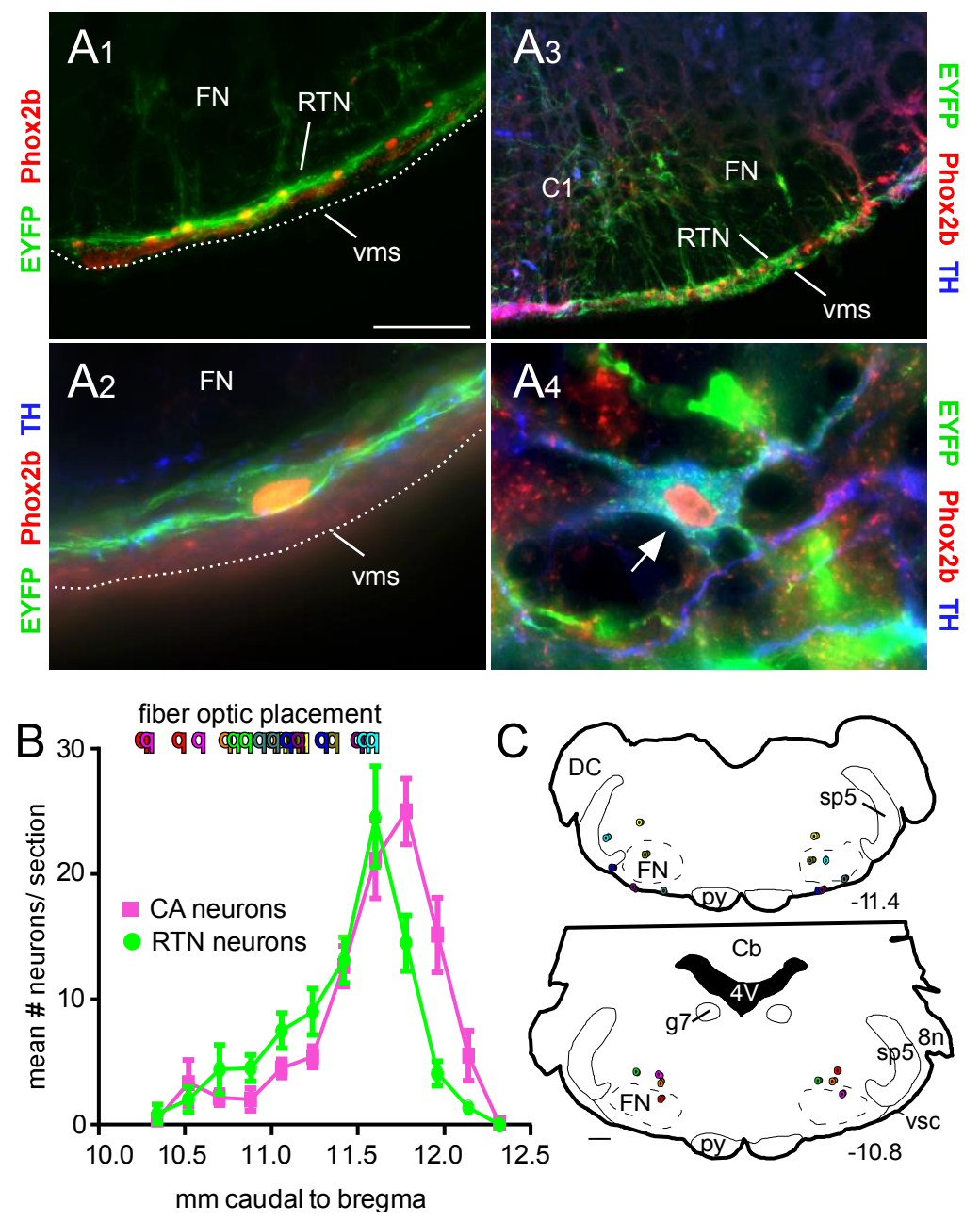
Figure 1: Location of Arch-transduced neurons and fiber optic tips.

A1. Coronal section through the medulla (level $\sim 11.4 \mathrm{~mm}$ caudal to bregma) illustrating the selective expression of ArchT-eYFP by RTN neurons. RTN is identified by the line of strongly Phox2b-ir nuclei close to the ventral medullary surface (Takakura et al., 2006). A2. Higher power photograph of an Arch-transduced RTN neuron. This neuron had a Phox2b-imunoreacttive (ir) nucleus, was negative for TH but had close appositions from TH-ir synaptic boutons. A3. Coronal section (level $\sim 11.7 \mathrm{~mm}$ caudal to bregma) showing transduced RTN neurons (on the ventral medullary surface) and catecholamine (CA, presumably C1) cells dorsal to the latter. A4. Example of a transduced CA neuron containing eYFP and TH immunoreactivity plus a Phox $2 \mathrm{~b}$-ir nucleus. Calibration bar in $\mathrm{A} 1=100 \mu \mathrm{m}$ in $\mathrm{A} 1,200 \mu \mathrm{m}$ in $\mathrm{A} 2$ and $20 \mu \mathrm{m}$ in $\mathrm{A} 2$ and $\mathrm{A} 4$. FN, facial motor nucleus, vms, ventral medullary surface. B. Rostrocaudal distribution of transduced RTN and CA neurons following bilateral injection of PRSx8-ArchT-eYFP lentiviral vector under the caudal end of the facial motor nucleus (30 $\mu \mathrm{m}$-thick sections, $180 \mu \mathrm{m}$ apart). Abbreviations: $8 \mathrm{n}$, $8^{\text {th }}$ nerve root; $\mathrm{Cb}$, cerebellum; DC, dorsal cochlear nucleus; FN, facial motor nucleus; $\mathrm{g} 7$, genu of the $7^{\text {th }}$ nerve; py, pyramidal tract; sp5, spinal trigeminal tract; vsc, ventral spinocerebellar tract. The FN extends from -11.6 to $-10 \mathrm{~mm}$ caudal to bregma. C. Location of the bilateral fiber optic tips identified in 10 rats. These sites are plotted on two transverse brain sections closest to their location, unique colors represent unique subjects. The exact transverse plane in which the fiber optic tips were found is also represented in panel B (triangles, same color code as in C). Stereotaxic coordinates (transverse planes posterior to bregma) correspond to the atlas of Paxinos and Watson ( 2005). 
Figure 2.

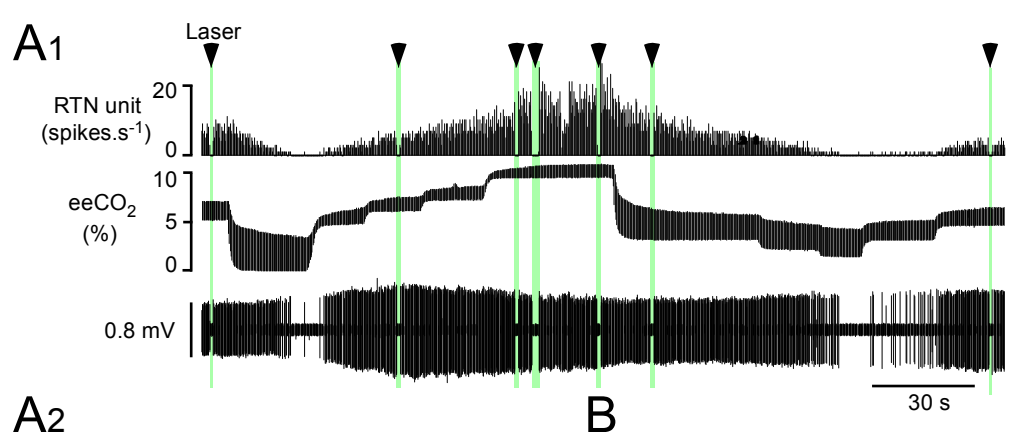

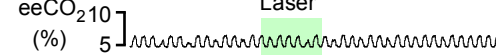

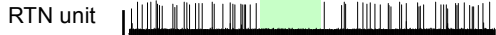

$$
\begin{aligned}
& \text { (0.8 mV) }
\end{aligned}
$$

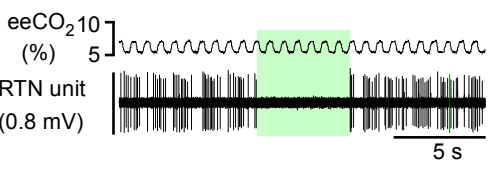

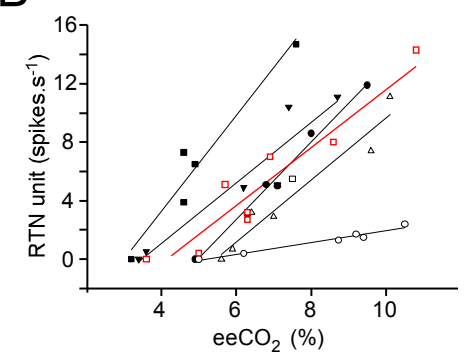


Figure 2: Arch photoactivation silences RTN neurons in anesthetized rats.

A1. Example of one extracellularly recorded RTN neuron. Top trace, integrated rate histogram (bin size: $1 \mathrm{~s}$ ); middle trace end-expiratory $\mathrm{CO}_{2}\left(e e \mathrm{CO}_{2}\right)$; lower trace, extracellular action potentials. End-expiratory $\mathrm{CO}_{2}$ was changed by adding variable concentrations of this gas to the breathing mixture. Laser $532 \mathrm{~nm}$ continuous light was delivered in the vicinity of the neuron via a fiberoptic (green bars and triangles). The light produced immediate and complete inhibition of the neuron; A2. Expanded scale excerpts showing that the neuron was instantly and reversibly silenced by the light under low (upper trace) or high end-expiratory $\mathrm{CO}_{2}$ (lower trace); $\mathrm{B}$. relationship between unit discharge rate and end-expiratory $\mathrm{CO}_{2}$ for 6 RTN units that were silenced by green light (the cell shown in $\mathrm{A} 1$ and $\mathrm{A} 2$ is represented in red). 
Figure 3.
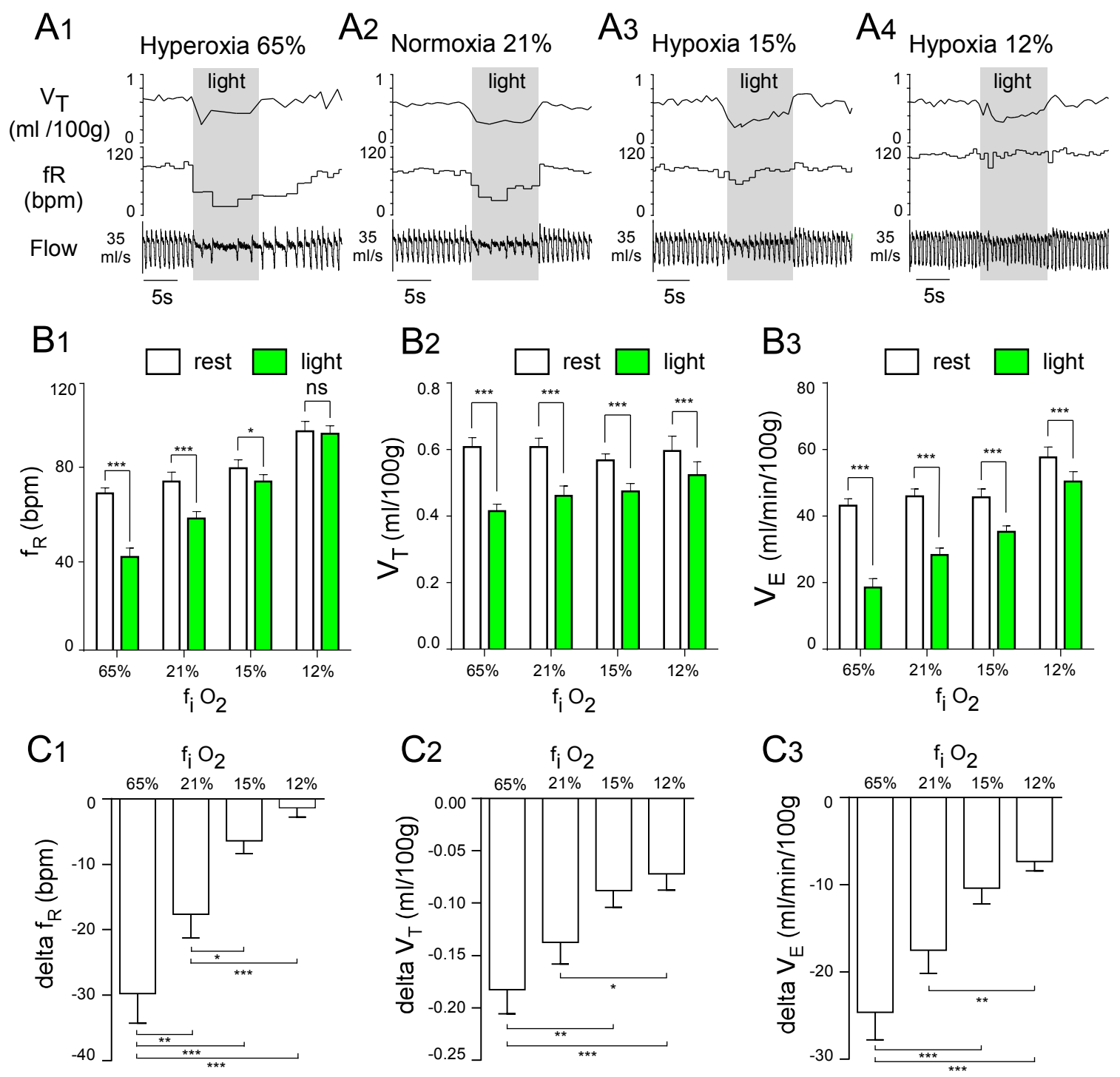
Figure 3: Arch photoactivation reduces breathing rate and amplitude to different extent in conscious rats exposed to normoxia, hyperoxia or hypoxia.

A. Hypoventilation caused by bilateral Arch photoactivation (10 s) in a conscious SpragueDawley rat under hyperoxia $\left(65 \% \mathrm{O}_{2}\right.$, panel $\left.\mathrm{A} 1\right)$, normoxia $\left(21 \% \mathrm{O}_{2}\right.$, panel $\left.\mathrm{A} 2\right)$, mild hypoxia $(15 \%$ $\mathrm{O}_{2}$, panel $\left.\mathrm{A} 3\right)$ and moderate hypoxia $\left(12 \% \mathrm{O}_{2}\right.$, panel $\left.\mathrm{A} 4\right)$; $\mathrm{B}$. Ventilation parameters at rest and during Arch photostimulation in rats exposed to four levels (percentages) of $\mathrm{O}_{2}$ in the breathing mixture $\left(\mathrm{FiO}_{2}\right)$ : group data (9 rats). B1. Breathing frequency $\left(\mathrm{f}_{\mathrm{R}}\right)$ at rest and during Arch photostimulation. Two-way RM ANOVA: effect of $\mathrm{FiO}_{2}$ on $\mathrm{f}_{\mathrm{R}}$ : $F_{3,24}=30.71, p<0.0001$; effect of stimulation on $\mathrm{f}_{\mathrm{R}}: F_{1,8}=36.91, p=0.0003$. Interaction effect on $\mathrm{f}_{\mathrm{R}}: F_{3,24}=32.04, p<0.0001$. Sidak's multiple comparison: $65 \% \mathrm{O}_{2}$ vs. $65 \% \mathrm{O}_{2}+$ light, $p<0.0001 ; 21 \% \mathrm{O}_{2}$ vs. $21 \% \mathrm{O}_{2}+$ light, $p$ $<0.0001 ; 15 \% \mathrm{O}_{2}$ vs. $15 \% \mathrm{O}_{2}+$ light, $p=0.0493$. Sidak's comparison of $12 \% \mathrm{O}_{2}$ vs. $12 \% \mathrm{O}_{2}+$ light, $p=0.9751$. B2. Tidal volume $\left(\mathrm{V}_{\mathrm{T}}\right)$ at rest and during Arch photostimulation. Two-way RM ANOVA: effect of $\mathrm{FiO}_{2}$ on $\mathrm{V}_{\mathrm{T}}: F_{3,24}=0.9629, p=.4263$; effect of stimulation on $\mathrm{V}_{\mathrm{T}}: F_{1,8}=72.17, p<$ 0.0001. Interaction effect on $\mathrm{V}_{\mathrm{T}}: F_{3,24}=19.67, p<0.0001$ ). Sidak's multiple comparison: $65 \% \mathrm{O}_{2}$ vs. $65 \% \mathrm{O}_{2}+$ light, $p<0.0001 ; 21 \% \mathrm{O}_{2}$ vs. $21 \% \mathrm{O}_{2}+$ light, $p<0.0001 ; 15 \% \mathrm{O}_{2}$ vs. $15 \% \mathrm{O}_{2}+$ light, $p$ $<0.0001 ; 12 \% \mathrm{O}_{2}$ vs. $12 \% \mathrm{O}_{2}+$ light, $p<0.0001$. B3. Minute ventilation $\left(\mathrm{V}_{\mathrm{E}}\right)$ at rest and during Arch photostimulation. Two-way RM ANOVA: effect of $\mathrm{FiO}_{2}$ on $\mathrm{V}_{\mathrm{E}}: F_{3,24}=23.13, p<0.0001$. Effect of stimulation on $\mathrm{V}_{\mathrm{E}}: F_{1,8}=77.01, p<0.0001$. Interaction effect on $\mathrm{V}_{\mathrm{E}}: F_{3,24}=35.94, p<0.0001$ ). Sidak's multiple comparison: $65 \% \mathrm{O}_{2}$ vs. $65 \% \mathrm{O}_{2}+$ light, $p<0.0001 ; 21 \% \mathrm{O}_{2}$ vs. $21 \% \mathrm{O}_{2}+$ light, $p$ $<0.0001 ; 15 \% \mathrm{O}_{2}$ vs. $15 \% \mathrm{O}_{2}+$ light, $p<0.0001 ; 12 \% \mathrm{O}_{2}$ vs. $12 \% \mathrm{O}_{2}+$ light, $p<0.0001 ; \mathrm{C}$. Change in ventilation parameters elicited by Arch photostimulation: group data (same 9 rats as in B, statistical analysis by one-way RM ANOVA). C1. Overall effect of $\mathrm{FiO}_{2}$ on $\mathrm{f}_{\mathrm{R}}: F_{3,24}=32.04, p<$ 0.0001. Bonferroni multiple comparison: $65 \% \mathrm{O}_{2}$ vs. $21 \% \mathrm{O}_{2}, p=0.0039 ; 65 \% \mathrm{O}_{2}$ vs. $15 \% \mathrm{O}_{2}, p<$ 
$0.0001 ; 65 \% \mathrm{O}_{2}$ vs. $12 \% \mathrm{O}_{2}, \mathrm{p}<0.0001 ; 21 \% \mathrm{O}_{2}$ vs. $15 \% \mathrm{O}_{2}, p=0.0102 ; 21 \%$ vs, $12 \% \mathrm{O}_{2}, p=$ 0.0002. C2. Overall effect of $\mathrm{FiO}_{2}$ on $\mathrm{V}_{\mathrm{T}}: F_{3,32}=8.854, p=0.0002$. Bonferroni multiple comparison test: $65 \% \mathrm{O}_{2}$ vs. $15 \% \mathrm{O}_{2}, p=0.0031 ; 65 \% \mathrm{O}_{2}$ vs. $12 \% \mathrm{O}_{2}, p=0.0003 ; 21 \%$ vs, $12 \% \mathrm{O}_{2}, p=0.0423$. C3. Overall effect of $\mathrm{FiO}_{2}$ on $\mathrm{V}_{\mathrm{E}}: F_{3,32}=14.17, p<0.0001$. Bonferroni multiple comparison: $65 \%$ $\mathrm{O}_{2}$ vs. $15 \% \mathrm{O}_{2}, p=0.0002 ; 65 \% \mathrm{O}_{2}$ vs. $12 \% \mathrm{O}_{2}, p<0.0001 ; 21 \%$ vs. $12 \% \mathrm{O}_{2}, p=0.0064 .{ }^{*} p<$ $0.05, * * p<0.01, * * * p<0.001$. 
Figure 4.
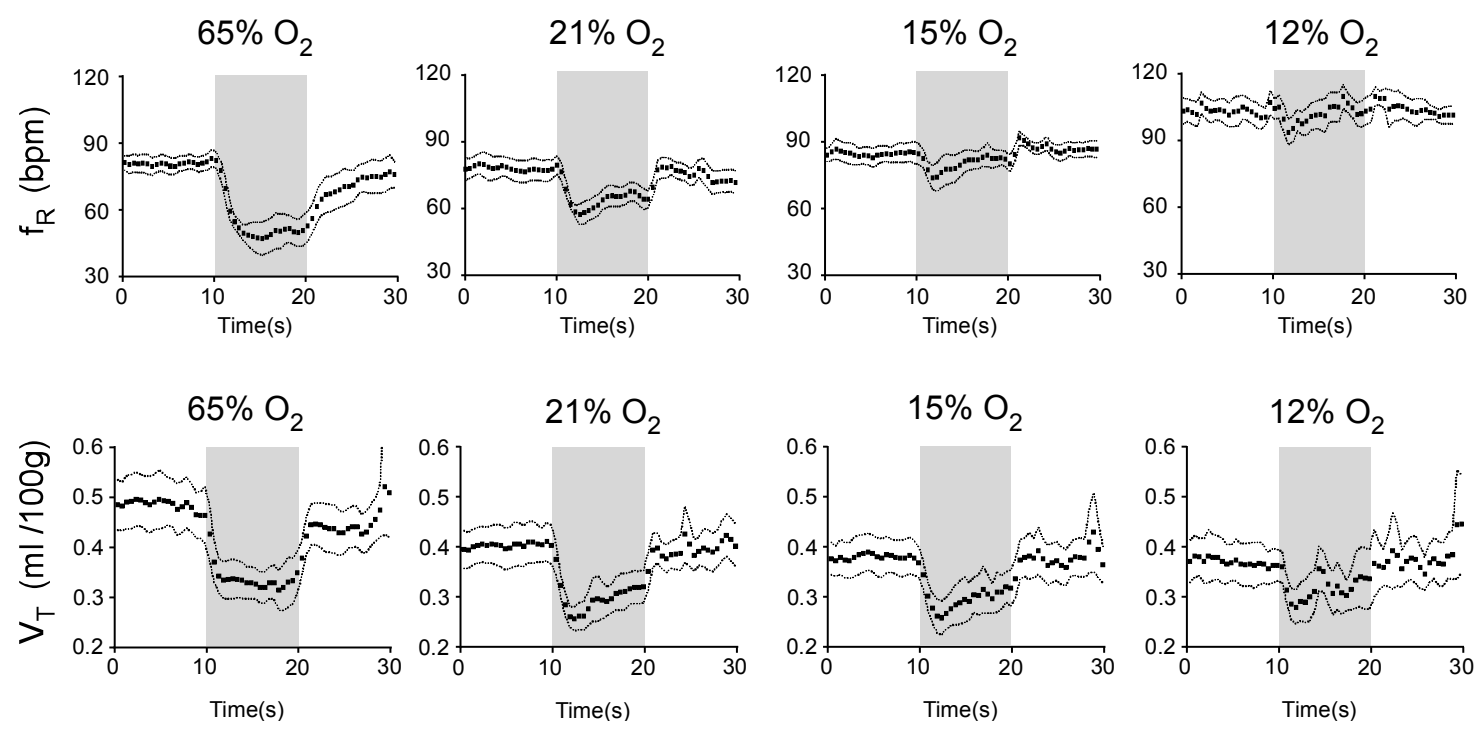
Figure 4: Kinetics of the hypoventilatory response at four different levels of inspired oxygen

Event-triggered averaged ventilatory responses to bilateral activation of Arch. Five to 6 responses were collected at each $\mathrm{FiO} 2$ in every rat $(\mathrm{N}=5)$ to generate a single average response per rat. Five such responses were averaged. The mean and $5 \%$ confidence intervals are depicted. 
Figure 5.
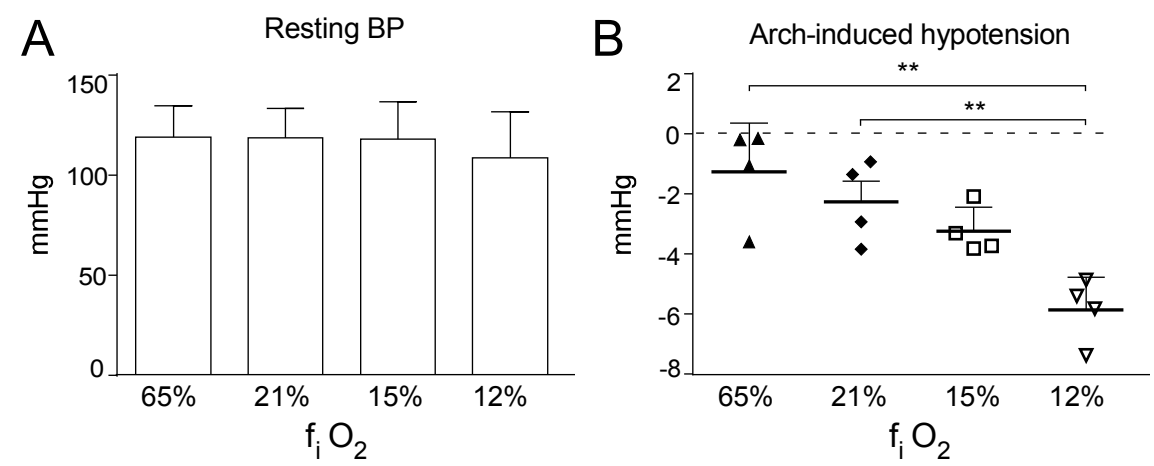
Figure 5: Effect of hypoxia on resting blood pressure and on the BP response to Arch stimulation

A. Blood pressure measurements at rest in 4 Sprague-Dawley rats under hyperoxia $\left(65 \% \mathrm{O}_{2}\right)$, normoxia $\left(21 \% \mathrm{O}_{2}\right)$, mild hypoxia $\left(15 \% \mathrm{O}_{2}\right)$ and moderate hypoxia $\left(12 \% \mathrm{O}_{2}\right)$. One-way RM ANOVA: effect of $\mathrm{FiO}_{2}$ on blood pressure: $F_{3,12}=0.2847, p=0.8356$; $\mathrm{B}$. Change in blood pressure parameters elicited by Arch photostimulation: group data (same 4 rats as in A, statistical analysis by one-way RM ANOVA). Overall effect of $\mathrm{FiO}_{2}$ on blood pressure: $F_{3,12}=10.05, p=0.0014$. Bonferroni multiple comparisons: $65 \% \mathrm{O}_{2}$ vs. $12 \% \mathrm{O}_{2}, p=0.0013,21 \%$ vs, $12 \% \mathrm{O}_{2}, p=0.0092 . * *$ $p<0.01$ 
Figure 6.

A

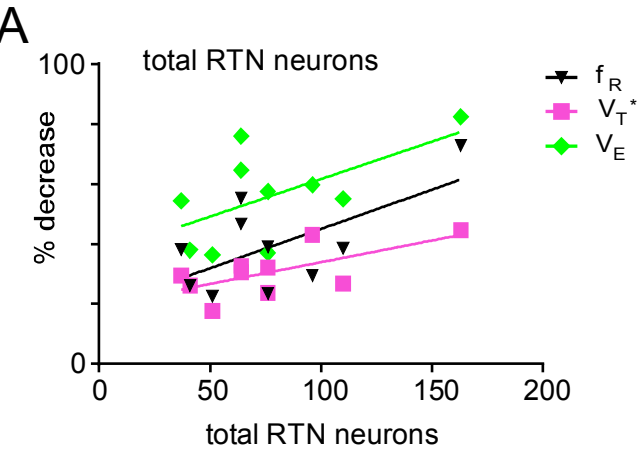

B

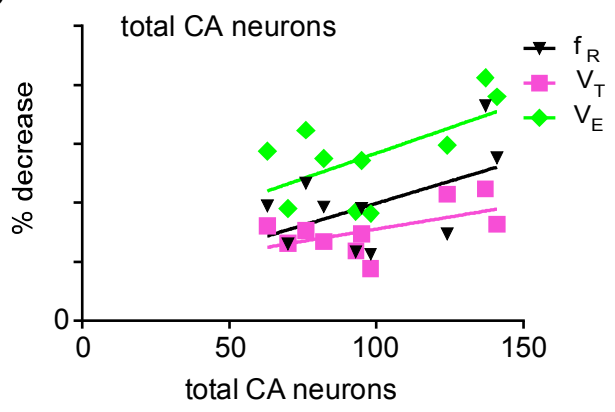

C

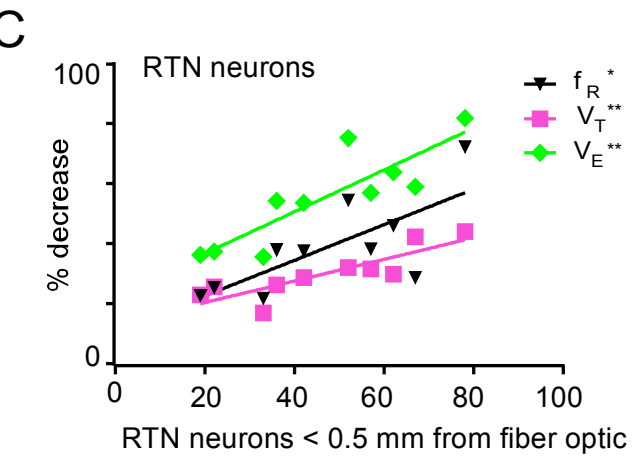

D

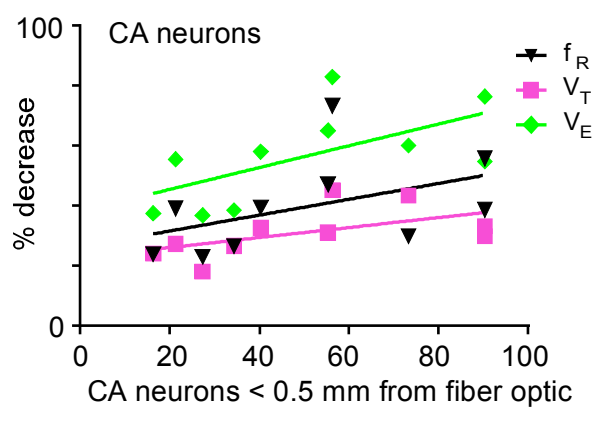


Figure 6: Relationship determined by linear regression between Arch-induced hypoventilation and the number of Arch-transduced neurons

A. Relationship between number of all RTN neurons transduced (counted in a 1:6 coronal series) and \% decrease of breathing frequency $\left(f_{R}\right)$, tidal volume $\left(V_{T}\right)$ and minute ventilation $\left(V_{E}\right)$ for 10 Sprague-Dawley rats elicited by Arch photostimulation. Percent decrease in $f_{R}: r^{2}=0.3900, F_{1,8}=$ 5.12, $p=0.0536$; \% decrease in $\mathrm{V}_{\mathrm{T}}: r^{2}=0.4366, F_{1,8}=6.20, p=0.0375$. Percent decrease in $\mathrm{V}_{\mathrm{E}}: r^{2}$ $=0.3519, F_{1,8}=4.344, p=0.071 ;$ B. Relationship between number of all catecholaminergic (CA) neurons transduced and \% decrease of $f_{R}, V_{T}$ and $V_{E}$ (for the same 10 Sprague-Dawley rats as in A) elicited by Arch photostimulation. Percent decrease in $\mathrm{f}_{\mathrm{R}}: r^{2}=0.2735, F_{1,8}=3.01, p=0.12 ; \%$ decrease in $\mathrm{V}_{\mathrm{T}}: r^{2}=0.3052, F_{1,8}=3.51, p=0.098$. Percent decrease in $\mathrm{V}_{\mathrm{E}}: r^{2}=0.3611, F_{1,8}=4.52$, $p=0.066$. C. Relationship between numbers of RTN neurons within $0.5 \mathrm{~mm}$ of the coronal plane containing the fiber optic tip. Percent decrease in $\mathrm{f}_{\mathrm{R}}: r^{2}=0.5413, F_{1,8}=9.44, p=0.0153 ; \%$ decrease in $\mathrm{V}_{\mathrm{T}}: r^{2}=0.7324, F_{1,8}=21.89, p=0.0016$. Percent decrease in $\mathrm{V}_{\mathrm{E}}: r^{2}=0.7435, F_{1,8}=$ 23.18, $p=0.0013$. D. Relationship between numbers of CA neurons within $0.5 \mathrm{~mm}$ of the coronal plane containing the fiber optic tip. Percent decrease in $\mathrm{f}_{\mathrm{R}}: r^{2}=0.275, F_{1,8}=2.095, p=0.1858 ; \%$ decrease in $\mathrm{V}_{\mathrm{T}}: r^{2}=0.2999, F_{1,8}=3.426, p=0.1013$. Percent decrease in $\mathrm{V}_{\mathrm{E}}: r^{2}=0.3859, F_{1,8}=$ 5.026, $p=0.0553 .{ }^{*} p<0.05,{ }^{* *} p<0.01$. 


\section{Figure 7.}
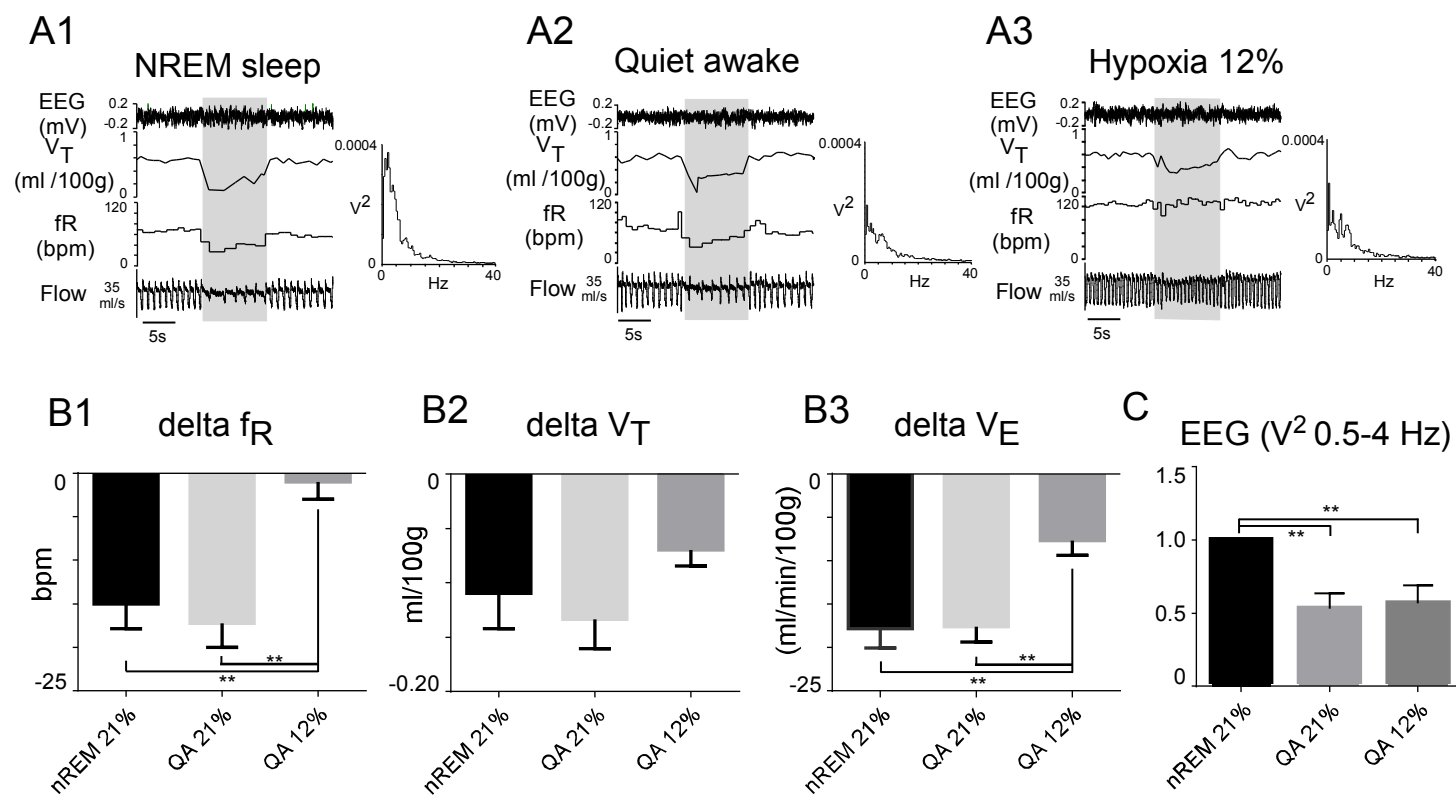

C
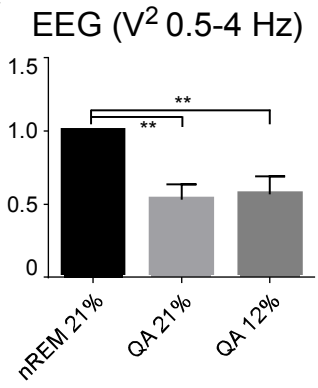
Figure 7: Arousal does not account for the reduction of Arch-triggered hypoventilation during hypoxia

A. Hypoventilation caused by bilateral Arch photoactivation (10 s, grey shaded bars) in one Sprague-Dawley rat during non-REM sleep (panel A1), quiet waking (panel A2) and hypoxia (12\% $\mathrm{FiO}_{2}$, panel A3). Each panel also depicts the EEG power (FFT, 0-40Hz) recorded during a $30 \mathrm{~s}$ window that included the photostimulus period and the $20 \mathrm{~s}$ preceding it; B. Group data (5 rats; statistics: one-way RM ANOVA). B1. Light evoked $\mathrm{f}_{\mathrm{R}}$ reduction during NREM sleep, quiet awake and hypoxia $\left(12 \% \mathrm{O}_{2}\right): F_{2,12}=11.79, p=0.0015$. Bonferroni multiple comparison: $21 \%$ QA vs. $12 \%$ QA, $p=0.0023 ; 21 \%$ nREM vs. $12 \%$ QA, $p=0.0068 . B 2 . V_{T}$ reduction during NREM sleep, quiet awake and hypoxia $\left(12 \% \mathrm{O}_{2}\right): F_{2,12}=1.556, p=0.2508$. B3. $\mathrm{V}_{\mathrm{E}}$ reduction during NREM sleep, quiet awake and hypoxia $\left(12 \% \mathrm{O}_{2}\right): F_{2,12}=9.136, p=0.0039$. Bonferroni multiple comparison: $21 \%$ QA vs. $12 \%$ QA, $p=0.0099 ; 21 \%$ nREM vs. $12 \%$ QA, $p=0.0083$; C. EEG power (0.5-4Hz only) during periods of quiet waking in normoxia or during hypoxia expressed as a fraction of the power recorded during periods of non-REM sleep (same 5 rats). One-way RM ANOVA: $F_{2,4}=$ $15.08, p=0.0165$. Bonferroni multiple comparison: NREM $21 \%$ vs. $21 \%$ QA, $p=0.0179 ; \mathrm{nREM}$ $21 \%$ vs. $12 \%$ QA, $p=0.0438 .{ }^{*} p<0.05,{ }^{* *} p<0.01$. 
Figure 8.
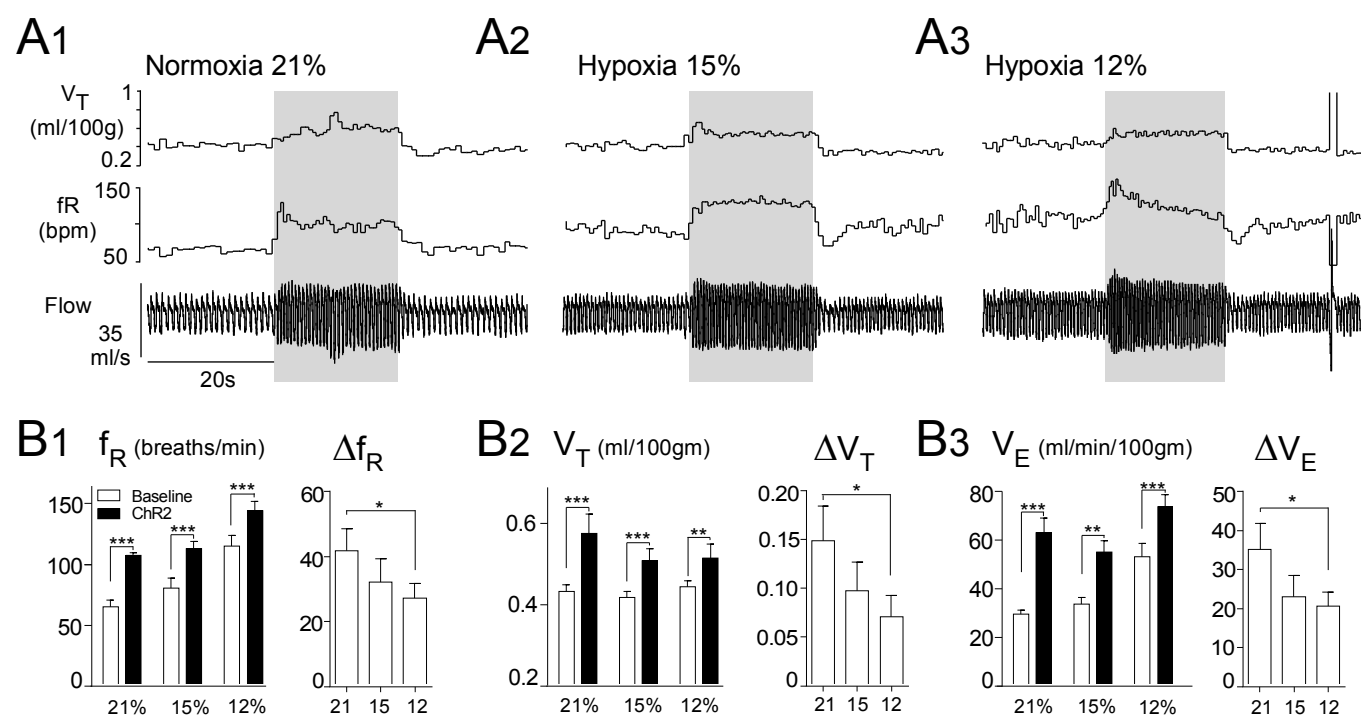

B3 $V_{E}(\mathrm{~m} / \mathrm{min} / 100 \mathrm{gm}) \quad \Delta \mathrm{V}_{E}$

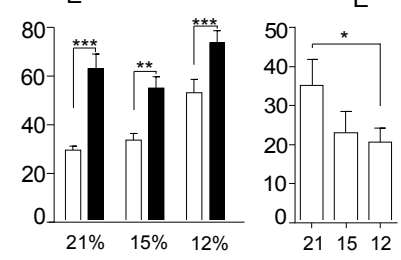


Figure 8:_Activation of channelrhodopsin (ChR2)-expressing RTN/C1 neurons increases breathing in normoxia and poikilocapnic hypoxia

A. Hyperventilation caused by unilateral ChR2 photoactivation ( $20 \mathrm{~s}, 20 \mathrm{~Hz}, 5 \mathrm{~ms}$ pulse width) in a conscious Sprague-Dawley rat under normoxia $\left(21 \% \mathrm{O}_{2}\right.$, panel $\left.\mathrm{A} 1\right)$, mild hypoxia $\left(15 \% \mathrm{O}_{2}\right.$, panel A2) and moderate hypoxia $\left(12 \% \mathrm{O}_{2}\right.$, panel A3); B. Left panels: ventilation parameters at rest and during ChR2 photostimulation in rats exposed to three levels (percentages) of $\mathrm{O}_{2}$ in the breathing mixture $\left(\mathrm{FiO}_{2}\right)$. Right panels: change in ventilation parameters evoked by ChR2 activation (6 rats). B1. Breathing frequency $\left(f_{R}\right)$. Statistical analysis: left panel, 2-way RM ANOVA. Effect of $\mathrm{FiO}_{2}$ on $\mathrm{f}_{\mathrm{R}}: F_{2,10}=35.46, p<0.0001$. Effect of stimulation on $\mathrm{f}_{\mathrm{R}}: F_{1,5}=37.39, p=0.0017$. Statistical analysis right panel: Friedman nonparametric one-way RM ANOVA: Friedman statistic $=9.333, p=0.0055$. Dunn's multiple comparison: $21 \% \mathrm{O}_{2}$ vs. $12 \% \mathrm{O}_{2}, p=0.0117$. Other Dunn's multiple comparisons not significant $p>0.05: 21 \% \mathrm{O}_{2}$ vs. $15 \% \mathrm{O}_{2} p=0.0628,15 \% \mathrm{O}_{2}$ vs. $12 \% \mathrm{O}_{2} p$ $=>0.9999$. B2. Tidal volume $\left(\mathrm{V}_{\mathrm{T}}\right)$ at rest and during ChR2 photostimulation. Statistical analysis for left panel: 2-way RMANOVA. Effect of $\mathrm{FiO}_{2}$ on $\mathrm{V}_{\mathrm{T}}: F_{2,10}=2.731, p=0.1132$. Effect of stimulation on $\mathrm{V}_{\mathrm{T}}: F_{1,5}=15.87, p=0.0105$. Statistical analysis for right panel by Friedman nonparametric one-way RM ANOVA: Friedman statistic $=9.333, p=0.0055$. Dunn's multiple comparison test: $21 \% \mathrm{O}_{2}$ and $12 \% \mathrm{O}_{2}, p=0.0117$. Other Dunn's multiple comparisons $p>0.05$ : $21 \% \mathrm{O}_{2}$ vs. $15 \% \mathrm{O}_{2} p=0.0628,15 \% \mathrm{O}_{2}$ vs. $12 \% \mathrm{O}_{2} p=>0.9999 . \mathrm{B} 3$. Minute ventilation $\left(\mathrm{V}_{\mathrm{E}}\right)$ at rest and during photostimulation. Left panel statistical analysis by 2-way RM ANOVA. Effect of $\mathrm{FiO}_{2}$ on $\mathrm{V}_{\mathrm{E}}: F_{2,10}=19.64, p=0.0003$; effect of stimulation on $\mathrm{V}_{\mathrm{E}}: F_{1,5}=28.61, p=0.0031$; interaction effect on $V_{E}: F_{2,10}=15.61, p=0.0008$. Statistical analysis for right panel by Friedman nonparametric one-way RM ANOVA: Friedman statistic $=9.333, p=0.0055$. Dunn's multiple comparison: $21 \% \mathrm{O}_{2}$ and $12 \% \mathrm{O}_{2}, p=0.0117$. Other Dunn's multiple comparisons not significant 
$p>0.05: 21 \% \mathrm{O}_{2}$ vs. $15 \% \mathrm{O}_{2} p=0.0628,15 \% \mathrm{O}_{2}$ vs. $12 \% \mathrm{O}_{2} p=>0.9999 ; .{ }^{*} \mathrm{p}<0.05,{ }^{* *} \mathrm{p}<0.01$, $* * * p<0.001$ 
Figure 9.
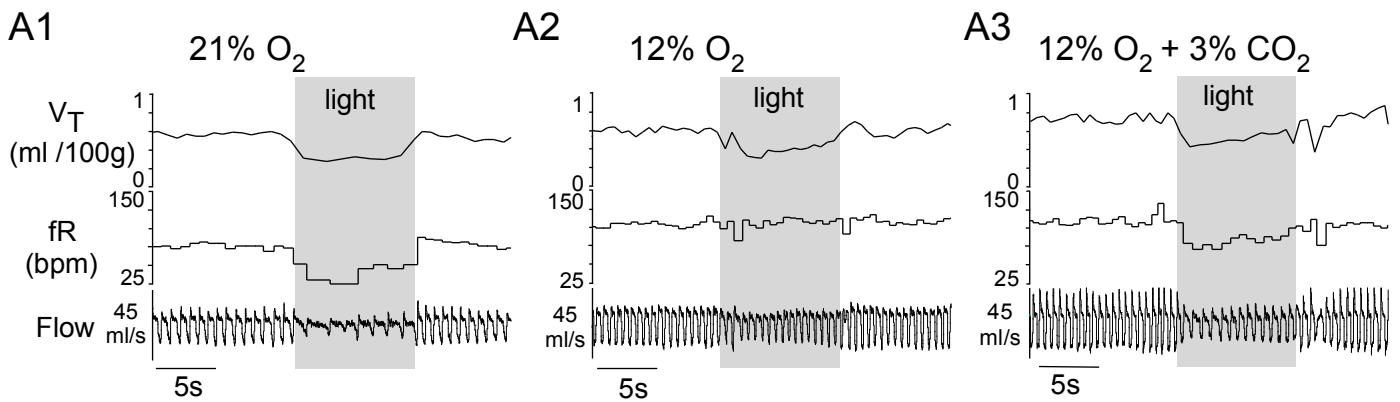

B1

B2

B3
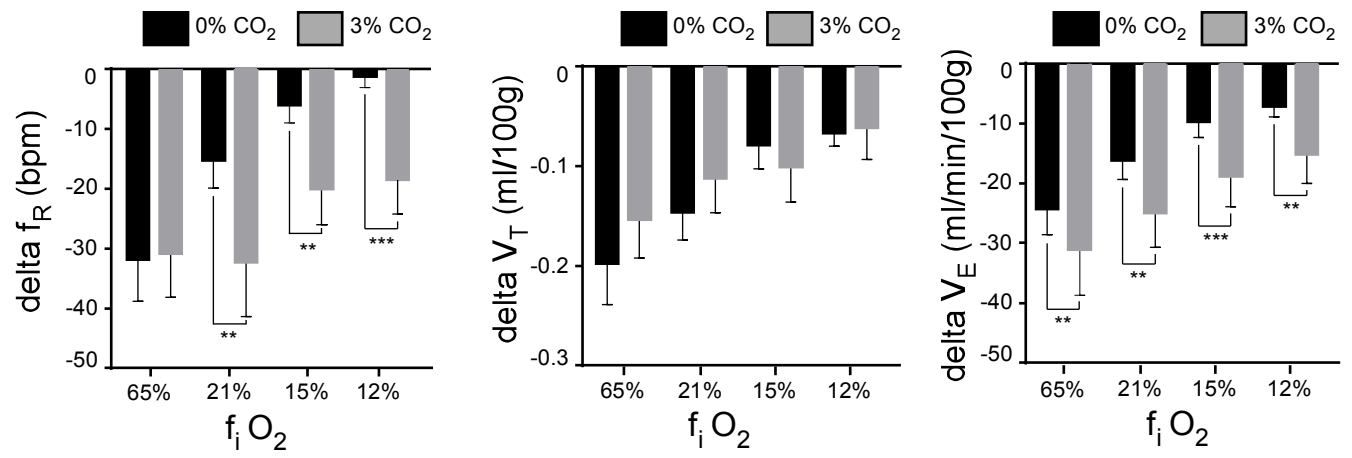
Figure 9: Hypercapnia restores the Arch-induced hypoventilation under hypoxia.

A. Sprague-Dawley rat in normoxia $\left(\mathrm{A} 1,21 \% \mathrm{O}_{2}\right)$, hypoxia $\left(12 \% \mathrm{O}_{2}\right)$ and during combined hypoxia $\left(12 \% \mathrm{O}_{2}\right)$ and hypercapnia $\left(3 \% \mathrm{CO}_{2}\right)$. Arch stimulation at grey shaded bars; $\mathrm{B}$. Hypoventilation elicited by Arch stimulation as a function of $\mathrm{FiO}_{2}$ and with the addition of $3 \% \mathrm{CO}_{2}$ : group data (5 rats, statistical analysis by 2-way RM ANOVA). B1. Change in breathing frequency $\left(f_{R}\right)$ at different ventilation parameters at rest and with $3 \% \mathrm{CO}_{2}$ during Arch photostimulation. Effect of stimulation during different levels of $\mathrm{FiO}_{2}$ on $\mathrm{f}_{\mathrm{R}}: F_{3,12}=16.11, p=0.0002$; effect of stimulation during different levels of $\mathrm{FiO}_{2}$ with $3 \% \mathrm{CO}_{2}$ on $\mathrm{f}_{\mathrm{R}}: F_{1,4}=11.58, p=0.0272$. Interaction effect on $\mathrm{f}_{\mathrm{R}}$ : $F_{3,12}=6.781, p=0.0063$. Sidak's multiple comparison: $21 \% \mathrm{O}_{2}$ vs. $21 \% \mathrm{O}_{2}+3 \% \mathrm{CO}_{2}, p=0.0010$ $15 \% \mathrm{O}_{2}$ vs. $15 \% \mathrm{O}_{2}+3 \% \mathrm{CO}_{2}, p=0.0048 ; 12 \% \mathrm{O}_{2}$ vs. $12 \% \mathrm{O}_{2}+3 \% \mathrm{CO}_{2} \mathrm{p}=0.0009$. Sidak's multiple comparison $p>0.05$ for $65 \% \mathrm{O}_{2}$ vs. $65 \% \mathrm{O}_{2}+3 \% \mathrm{CO}_{2}, p=0.9976$. B2. Change in tidal volume $\left(\mathrm{V}_{\mathrm{T}}\right)$ at different ventilation parameters at rest and with $3 \% \mathrm{CO}_{2}$ during Arch photostimulation. Effect of stimulation during different levels of $\mathrm{FiO}_{2}$ on $\mathrm{V}_{\mathrm{T}}: F_{3,12}=11.53, p=$ 0.0008; effect of stimulation during different levels of $\mathrm{FiO}_{2}$ with $3 \% \mathrm{CO}_{2}$ on $\mathrm{V}_{\mathrm{T}}: F_{1,4}=1.418, p=$ 0.2996. Interaction effect on $\mathrm{V}_{\mathrm{T}}: F_{3,12}=2.413, p=0.1174$. Sidak's multiple comparison $p>0.05$ for all comparisons: $65 \% \mathrm{O}_{2}$ vs. $65 \% \mathrm{O}_{2}+3 \% \mathrm{CO}_{2}, p=0.1464 ; 21 \% \mathrm{O}_{2}$ vs. $21 \% \mathrm{O}_{2}+3 \% \mathrm{CO}_{2}, p=$ $0.3554 ; 15 \% \mathrm{O}_{2}$ vs. $15 \% \mathrm{O}_{2}+3 \% \mathrm{CO}_{2}, p=0.7281 ; 12 \% \mathrm{O}_{2}$ vs. $12 \% \mathrm{O}_{2}+3 \% \mathrm{CO}_{2} \mathrm{p}=0.9975 . \mathrm{B} 3$. Change in minute ventilation $\left(\mathrm{V}_{\mathrm{E}}\right)$ at different ventilation parameters at rest and with $3 \% \mathrm{CO}_{2}$ during Arch photostimulation. Effect of stimulation during different levels of $\mathrm{FiO}_{2}$ on $\mathrm{V}_{\mathrm{E}}: F_{3,12}=$ $16.59, p=0.0001$; effect of stimulation during different levels of $\mathrm{FiO}_{2}$ with $3 \% \mathrm{CO}_{2}$ on $\mathrm{V}_{\mathrm{E}}: F_{1,4}=$ 7.715, $p=0.0499$. Interaction effect on $\mathrm{V}_{\mathrm{E}}: F_{3,12}=0.3424, p=0.7952$. Sidak's multiple comparison test: $65 \% \mathrm{O}_{2}$ vs. $65 \% \mathrm{O}_{2}+3 \% \mathrm{CO}_{2}, p=0.0079 ; 21 \% \mathrm{O}_{2}$ vs. $21 \% \mathrm{O}_{2}+3 \% \mathrm{CO}_{2}, p=$ 
$0.0011 ; 15 \% \mathrm{O}_{2}$ vs. $15 \% \mathrm{O}_{2}+3 \% \mathrm{CO}_{2}, p=0.0008 ; 12 \% \mathrm{O}_{2}$ vs. $12 \% \mathrm{O}_{2}+3 \% \mathrm{CO}_{2}, p=0.0025 .{ }^{* *} p$ $<0.01, * * * p<0.001$. 
Figure 10.

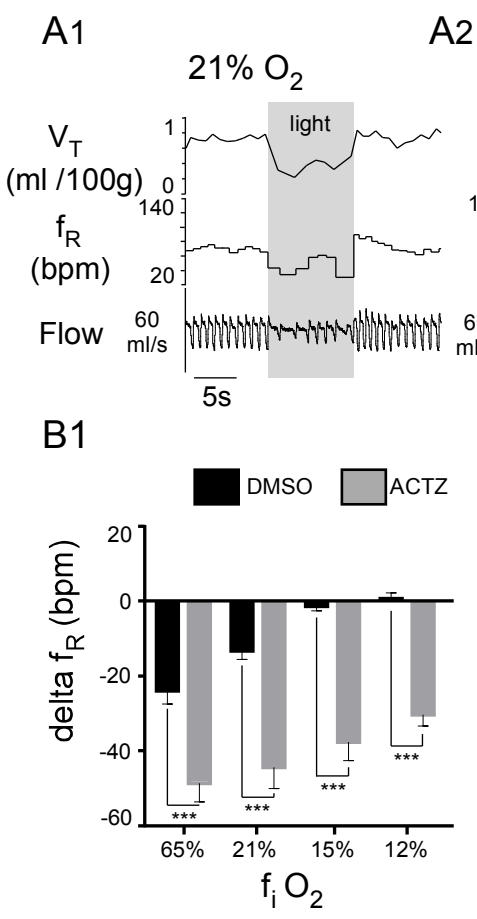

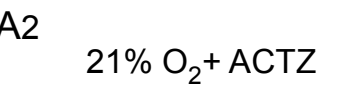

A3 $12 \% \mathrm{O}_{2}$

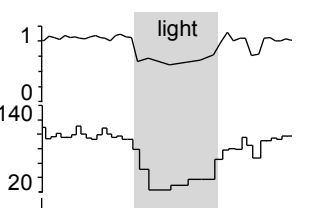

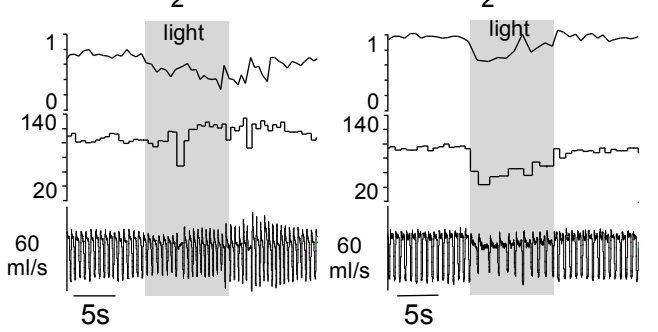

B2

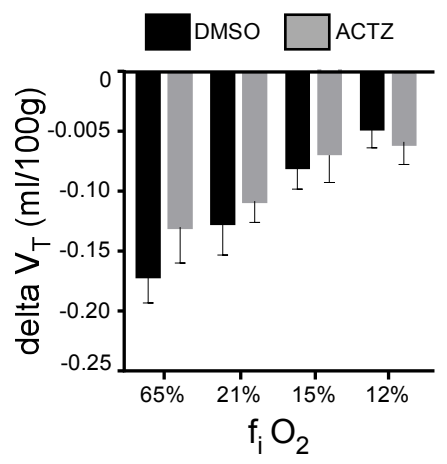

B3

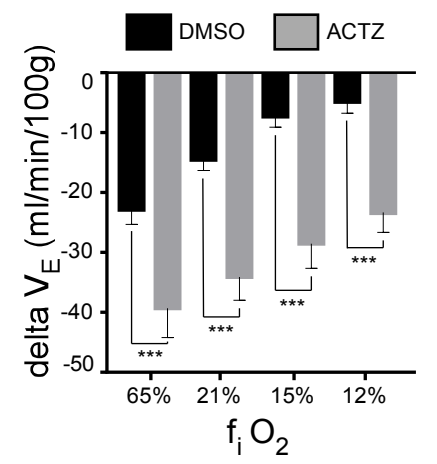


Figure 10: Acetazolamide restores the Arch-induced hypoventilation under hypoxia.

A1, A2. A Sprague-Dawley rat in a normoxic $\left(21 \% \mathrm{O}_{2}\right)$ condition and with the addition of acetazolamide respectively before, during and after Arch stimulation of the transduced RTN neurons (laser on for 10s shown by grey shaded regions); A3, A4. The same rat under hypoxia $\left(12 \% \mathrm{O}_{2}\right)$ and with acetazolamide before, during and after Arch stimulation; B. Change from baseline in frequency of respiration, tidal volume and minute ventilation under the different levels of $\mathrm{O}_{2}$ during Arch photostimulation compared to the change evoked during Arch photostimulation with acetazolamide under each $\mathrm{O}_{2}$ condition $(\mathrm{N}=6$, Control $=7)$. B1. Change in breathing frequency $\left(f_{R}\right)$ at different ventilation parameters at rest and with acetazolamide during Arch photostimulation. Effect of stimulation during different levels of $\mathrm{FiO}_{2}$ on $\mathrm{f}_{\mathrm{R}}: F_{3,15}=$ 53.40, $p=<0.0001$; effect of stimulation during different levels of $\mathrm{FiO}_{2}$ with acetazolamide on $\mathrm{f}_{\mathrm{R}}$ : $F_{1,5}=93.01, p=0.0002$. Interaction effect on $\mathrm{f}_{\mathrm{R}}: F_{3,15}=2.335, p=0.1151$. Sidak's multiple comparison: $65 \% \mathrm{O}_{2}$ vs. $65 \% \mathrm{O}_{2}+$ acetazolamide, $p<0.0001 ; 21 \% \mathrm{O}_{2}$ vs. $21 \% \mathrm{O}_{2}+$ acetazolamide, $p<0.0001 ; 15 \% \mathrm{O}_{2}$ vs. $15 \% \mathrm{O}_{2}+$ acetazolamide, $p=0.0001 ; 12 \% \mathrm{O}_{2}$ vs. $12 \% \mathrm{O}_{2}+$ acetazolamide, $p<0.0001$. B2. Change in tidal volume $\left(\mathrm{V}_{\mathrm{T}}\right)$ at different ventilation parameters at rest and with acetazolamide during Arch photostimulation. Effect of stimulation during different levels of $\mathrm{FiO}_{2}$ on $\mathrm{V}_{\mathrm{T}}: F_{3,15}=52.70, p<0.0001$; effect of stimulation during different levels of $\mathrm{FiO}_{2}$ with acetazolamide on $\mathrm{V}_{\mathrm{T}}: F_{1,5}=4.408, p=0.0898$. Interaction effect on $\mathrm{V}_{\mathrm{T}}: F_{3,15}=1.108, p=$ 0.3768. Sidak's multiple comparison test $p>0.05$ for differences: $65 \% \mathrm{O}_{2}$ vs. $65 \% \mathrm{O}_{2}+$ acetazolamide, $p=0.1435 ; 21 \% \mathrm{O}_{2}$ vs. $21 \% \mathrm{O}_{2}+$ acetazolamide, $p=4941 ; 15 \% \mathrm{O}_{2}$ vs. $15 \% \mathrm{O}_{2}+$ acetazolamide, $p=9259 ; 12 \% \mathrm{O}_{2}$ vs. $12 \% \mathrm{O}_{2}+$ acetazolamide, $p=0.9996$. B3. Change in minute ventilation $\left(\mathrm{V}_{\mathrm{E}}\right)$ at different ventilation parameters at rest and with acetazolamide during Arch photostimulation. Effect of stimulation during different levels of $\mathrm{FiO}_{2}$ on $\mathrm{V}_{\mathrm{E}}: F_{3,15}=62.22, p=$ 
$<0.0001$; effect of stimulation during different levels of $\mathrm{FiO}_{2}$ with acetazolamide on $\mathrm{V}_{\mathrm{E}}: F_{1,5}=$ 32.73, $p=0.0023$. Interaction effect on $\mathrm{V}_{\mathrm{E}}: F_{3,15}=1.198, p=0.3442$. Sidak's multiple comparisons : $65 \% \mathrm{O}_{2}$ vs. $65 \% \mathrm{O}_{2}+$ acetazolamide, $p<0.0001 ; 21 \% \mathrm{O}_{2}$ vs. $21 \% \mathrm{O}_{2}+$ acetazolamide, $p<$ $0.0001 ; 15 \% \mathrm{O}_{2}$ vs. $15 \% \mathrm{O}_{2}+$ acetazolamide, $p<0.0001 ; 12 \% \mathrm{O}_{2}$ vs. $12 \% \mathrm{O}_{2}+$ acetazolamide, $p$ $<0.0001) . * * * p<0.001$ 
Figure 11.
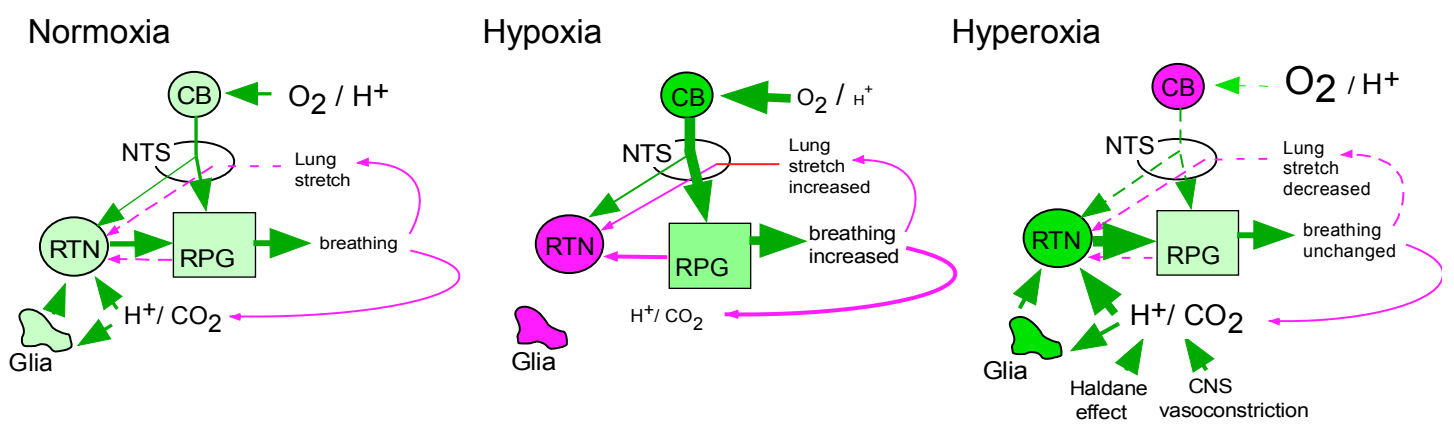
Figure 11: Summary and interpretations.

During eupnea (regular involuntary breathing as during NREM sleep and quiet waking) breathing rate and amplitude are generated by a network of pontomedullary structures referred to as the respiratory pattern generator (RPG). Under normoxia, eupneic breathing is driven to a considerable extent by excitatory inputs (green arrows) that originate from the RTN and from the carotid bodies, CBs. The CB pathway relays in the nucleus of the solitary tract (NTS). RTN receives an excitatory input from the $\mathrm{CBs}$ but, as shown here by the hypoxia experiments, the CBs also activate the RPG via pathways that bypass the RTN. RTN neurons are subject to several feedback regulations (red lines). The most powerful one operates via $\mathrm{CO}_{2} / \mathrm{pH}$ and is largely mediated by the withdrawal of the direct and astrocyte-dependent excitatory effect of acid on RTN neurons. Additional feedbacks (red lines) originate from lung stretch receptors and the RPG and may protect the lungs against hyperinflation.

During poikilocapnic hypoxia (low $\mathrm{FiO}_{2}$, no $\mathrm{CO}_{2}$ supplementation), the $\mathrm{CBs}$ are highly active, drive the RPG vigorously which increases breathing and lowers arterial $\mathrm{PCO}_{2}$ and $\mathrm{H}^{+}$. The increased ventilation further activates the inhibitory feedbacks to RTN (solid red lines) causing these cells to become silent, the chemical feedback (via alkalization of the RTN) being the most powerful. This feedback causes RTN neurons to become silent under hypoxia despite an increase in excitatory input from the CBs.

During hyperoxia, the $\mathrm{CBs}$ are inactive. $\mathrm{H}^{+}$and $\mathrm{CO}_{2}$ accumulate at the level of the RTN for at least two reasons. The loss of $\mathrm{CB}$ activity reduces ventilation (in rats only) and hyperoxia reduces cerebral blood flow and facilitates $\mathrm{CO}_{2}$ dumping from erythrocytes (Haldane effect), causing brain $\mathrm{PCO}_{2}$ to rise. The resulting brain acidification activates the RTN further and, 
because of the loss of the respiratory drive that originates from the CBs, RTN contributes a much larger portion of the respiratory drive than under normoxia. Additional CRC candidates (serotonergic, orexinergic neurons etc.) are not represented. These CRCs conceivably operate along the same principles during changes in inhaled oxygen.

Color coding: green arrows, excitatory pathways or stimulatory effects. Weak inputs are represented as dashed lines, stronger input as solid lines of increasing thickness. Magenta arrows: inhibitory pathways or feedbacks (intensity also coded with dashes and lines of increasing thickness). Pale green circles or boxes: moderately active cell groups; dark green, high activity; magenta, inactivity (as in CBs during hyperoxia).

Author Contributions: P.G.G., R.L.S., T.M.B., P.G.R.B. conception and design of research; T.M.B., P.G.R.B, R.K., K.E.V., D.S.S. performed experiments; T.M.B., P.G.R.B., R.L.S. analyzed data; T.M.B., P.G.R.B., P.G.G. prepared figures; P.G.G., T.M.B drafted manuscript; P.G.G., T.M.B, P.G.R.B., R.L.S. edited and revised manuscript; P.G.G., T.M.B., P.G.R.B., R.L.S. interpreted results of experiments; P.G.G. approved final version of manuscript. 


\section{Chapter 3: State-dependent control of breathing by the retrotrapezoid nucleus}

\section{This chapter is a re-print of the Journal of Physiology (2015) article (Burke et al.,} 2015a).

\subsection{Introduction}

The retrotrapezoid nucleus is a small cluster of lower brainstem neurons that are activated by hypercapnia and regulate several aspects of breathing including inspiratory amplitude, breathing frequency and active expiration (Marina et al., 2010; Guyenet, 2014a; Basting et al., 2015). The high responsiveness of RTN neurons to $\mathrm{CO}_{2}$ in vivo is attributed to several mechanisms: an intrinsic sensitivity to protons, paracrine effects from surrounding $\mathrm{pH} / \mathrm{CO}_{2}$-sensitive astrocytes and inputs from other chemosensory neurons and the carotid bodies (Guyenet et al., 2005; Gestreau et al., 2010; Gourine et al., 2010; Guyenet et al., 2010; Huckstepp \& Dale, 2011; Ramanantsoa et al., 2011; Wang et al., 2013a). RTN neurons can therefore be viewed as central respiratory chemoreceptors (CRCs) that also function as chemoreflex integrator. These neurons mediate a large portion of the hypercapnic ventilatory reflex (HCVR) (Gestreau et al., 2010; Guyenet et al., 2010; Ramanantsoa et al., 2011; Wang et al., 2013a).

The chemoreflex control of breathing is state-dependent. For example, although the respiratory chemoreflexes still operate during REM sleep, the breathing frequency $\left(f_{R}\right)$ is typically unaffected by hypoxia or hypercapnia (Coote, 1982; Berthon-Jones \& Sullivan, 1984; Horner et al., 2002; Lovering et al., 2003; Nakamura et al., 2007). Also, in conscious cats 
ventilated to apnea during non-REM sleep, diaphragmatic EMG reemerges during REM sleep (Orem et al., 2005).

Periodic breathing occurs during sleep under hypobaric hypoxia, in congestive heart failure (CHF) patients, or after chronic opiate treatment (Berssenbrugge et al., 1983; Burgess, 1997; Farney et al., 2003; Weil, 2004). The apneas have been tentatively attributed to recurrent episodes of CNS hypocarbia that silence CRCs (Dempsey et al., 2012; Marcus et al., 2014). This interpretation agrees with the finding that respiratory alkalosis silences RTN in conscious rats (Basting et al., 2015). In adult man or cat, periodic breathing occurs only during non-REM sleep (Berssenbrugge et al., 1983; Farney et al., 2003; Lovering et al., 2012).

Congenital central hypoventilation syndrome (CCHS) is caused by Phox $2 b$ mutations (Amiel et al., 2003; Weese-Mayer et al., 2010). This disease is characterized by the loss of breathing automaticity during sleep and a greatly reduced HCVR (Amiel et al., 2003; WeeseMayer et al., 2010). In mice, Phox $2 b$ mutations abort RTN development, which probably causes most of the HCVR deficit (Ramanantsoa et al., 2011). In CCHS patients, breathing is much better maintained during REM than non-REM sleep (Fleming et al., 1980). In short, reduced CRC activity resulting from carotid body hyperactivity (hypoxia, CHF)(Marcus et al., 2014) or RTN lesion (putative cause of CCHS) appears to cause central sleep apnea (CSA) selectively during non-REM sleep.

The purpose of this study was to examine the state-dependence of the HCVR. We simulated an instantaneous increase or decrease of CRC activity by activating or inhibiting the retrotrapezoid nucleus optogenetically (Abbott et al., 2009a; Basting et al., 2015). We then 
measured the resulting effects on breathing during periods of quiet wake, non-REM or REM sleep.

\subsection{Materials \& Methods}

\section{Animals}

Experiments were performed on male Sprague-Dawley rats ( $N=28 ; 400-550$ gm, Taconic, USA). All procedures conformed to the NIH Guide for the Care and Use of Laboratory Animals and were approved by the University of Virginia Animal Care and Use Committee. Animals were housed under standard $12 \mathrm{~h}$ light/dark cycle with ad libitum access to food and water.

\section{Lentiviral constructs and vector preparation}

This study used lentiviral vectors (LVV) that express their transgene under the control of the Phox2b-responsive artificial promoter PRSx8 (Hwang et al., 2001). When injected into the rostral ventrolateral medulla or ventrolateral pons (noradrenergic "A5" area) as described in this study, these LVVs transduce Phox2b-expressing neurons that are virtually exclusively catecholaminergic (C1 and A5 neurons) and RTN neurons (Abbott et al., 2009a). One of the LVVs (PRSx8-ChR2-mCherry) encoded the photoactivatable cation channel channelrhodopsin-2 (ChR2, H134R) fused to mCherry (Abbott et al., 2009a) and the other the photoactivatable proton pump archaerhodopsin (ArchT3.0) fused to eYFP (PRSx8-Arch-eYFP) (Han et al., 2011; Mattis et al., 2012; Basting et al., 2015). The vectors were produced by the University of North Carolina virus core and diluted to a final titer of $3.0 \times 10^{8}$ viral particles per $\mathrm{ml}$ with sterile PBS. We verified that the above viral dilution transduced Phox $2 \mathrm{~b}$-expressing neurons selectively (Figure 9). 


\section{Injections of virus and instrumentation}

We used two different approaches for targeting Arch or ChR2 to RTN neurons: Arch was delivered bilaterally to transduce RTN neurons en masse in both rostral and caudal regions and the optical fibers were targeted to the caudal RTN to illuminate the region with the highest density of RTN neurons (Takakura et al., 2008a). By contrast, we restricted ChR2 expression to the rostral portion of the RTN on the left side. This was done in order to photoactivate RTN neurons without involving the $\mathrm{C} 1$ neurons whose photoactivation produces arousal and also boost breathing via glutamatergic mechanisms (Abbott et al., 2013b; Abbott et al., 2014; Burke et al., 2014).

For LVV injection, the rats were anesthetized with a mixture of ketamine $(75 \mathrm{mg} / \mathrm{kg})$, xylazine $(5 \mathrm{mg} / \mathrm{kg}$ ) and acepromazine $(1 \mathrm{mg} / \mathrm{kg}$ ) given intraperitoneally (i.p.). The correct plane of anesthesia was assessed by the absence of the corneal and hind-paw withdrawal reflexes. Additional anesthetic was administered as necessary during surgery ( $25 \%$ of the original dose, i.p. or intramuscular). Body temperature was kept close to $37^{\circ} \mathrm{C}$ with a servo-controlled heating pad and a blanket. Rats received post-operative ampicillin (125 mg/kg, i.p.), and ketoprofen (3-5 $\mathrm{mg} / \mathrm{kg}$, subcutaneous (s.c.) for two consecutive days. All surgical procedures were performed under aseptic conditions. The hair over the skull, neck and cheek were removed and skin disinfected. An incision over the mandible was made to expose the facial nerve for antidromic activation of facial motor neurons. The rat was then placed prone on a stereotaxic apparatus (bite bar set at -3.5 mm for flat skull; David Kopf Instruments). A $1.5 \mathrm{~mm}$ diameter hole was drilled into the occipital plate on the left side (ChR2) or both sides (Arch) caudal to the parietooccipital suture. Viral solutions were loaded into a $1.2 \mathrm{~mm}$ internal diameter glass pipette 
broken to a 25 micron tip (external diameter). To target the rostral RTN region with ChR2, the pipette was inserted at a 12-14 degree angle pointing towards the front of the animal and injections of 100-140 $\mathrm{nl}$ were made at two sites separated $200 \mu \mathrm{m}$ (max. $280 \mathrm{nl}$ ). These sites were located $100-200 \mu \mathrm{m}$ below the rostral end of the facial motor nucleus whose lower edge was identified in each rat by mapping antidromic evoked potentials elicited by stimulating the facial nerve (Brown \& Guyenet, 1985). The Arch-LVV was injected bilaterally into four sites separated by $200 \mu \mathrm{m}$ (total volume $400-600 \mathrm{nl} /$ side), extending rostrally from the caudal end of the facial motor nucleus.

We then implanted electrodes for recording the electroencephalogram (EEG), neck electromyogram (EMG) and optical fiber-ferrule assemblies for light stimulation (Sparta et al., 2012). For EEG recordings, stainless steel jeweler screws (Plastics One) were implanted extradurally $(0.5-1 \mathrm{~mm}$ anterior, $1 \mathrm{~mm}$ lateral to bregma and $3 \mathrm{~mm}$ posterior, $2-2.5 \mathrm{~mm}$ lateral to bregma above the contralateral hemisphere). Two additional screws were implanted for structural support of the head stage and for grounding. Teflon-coated braided stainless steel wire (A-M systems) was stripped at the tip and wrapped around the implanted screws. Two additional wires were stripped at the tips and implanted in the superficial muscles of the neck for EMG recordings of postural activity. All wires were crimped to amphenol pins (A-M systems) and inserted into a plastic headstage (Plastics One). Implantable optical fibers $(230 \mu \mathrm{m}$, numerical aperture 0.39; Thorlabs) were constructed using published methods (Sparta et al., 2012) and stereotaxically directed into the ventrolateral medulla $0.5-0.8 \mathrm{~mm}$ dorsal to the LVV injection sites. The head-stage and optical fiber-ferrule assemblies were secured to the skull using a 2-part epoxy (Loctite). Incisions were then closed in two layers (muscle and skin) with absorbable sutures and vet bond adhesive. Rats recovered for a minimum of four weeks before 
experiments were conducted. A subset of animals were then implanted with radio-telemetry probes (PA-C10, Datasciences International, Saint Paul, MN) to record blood pressure from the descending aorta via the right femoral artery. Animals were left to recover for at least another week before physiological experiments began.

\section{Physiological experiments in freely-behaving rats}

Rats were first habituated to the testing environment which consisted of a Buxco unrestrained plethysmography chamber modified to allow tethered EEG/EMG recordings and optical stimulation. This equipment was visually-isolated and placed in low-ambient noise conditions. On the day of the experiment, rats were lightly anesthetized with isoflurane (induction with $5 \%$, maintenance with $2 \%$ in pure oxygen for $<1 \mathrm{~min}$ ) to permit cleaning of hardware and connections to the ferrule and EEG/EMG recording assembly. A $200 \mu$ m-thick multimode optical fiber was terminated with a ferrule and mated to the implanted ferrule with a zirconia sleeve. Optical matching gel (Fiber Instrument Sales) was applied at the ferrule junction to minimize light loss. A minimum of $1 \mathrm{hr}$ was allowed for recovery from anesthesia and the emergence of stable sleep patterns. Recordings were made between 12 pm- 6 pm, over multiple days, with a minimum of three days' rest between tests. The ventilatory response to RTN photoinhibition or activation was assessed using barometric, unrestrained whole-body plethysmography (EMKA technologies). The plethysmography chamber was continuously flushed with $1.5 \mathrm{I} / \mathrm{min}$ of $21 \% \mathrm{O}_{2}$ balanced with $\mathrm{N}_{2}$. Three or $6 \% \mathrm{CO}_{2}$ was added when required, leaving the oxygen percentage constant. Photoinhibition of RTN under hyperoxia $\left(65 \% \mathrm{O}_{2}\right.$ in $\left.\mathrm{N}_{2}\right)$ was also performed, as previously described (Basting et al., 2015). The gas composition was 
regulated by computer-driven mass flow controllers for $\mathrm{O}_{2}, \mathrm{~N}_{2}$ and $\mathrm{CO}_{2}$ (Alicat). Temperature and humidity within the plethysmography chamber were kept constant.

\section{Optogenetics}

Photoinhibition of Arch-expressing RTN neurons was achieved with a green laser (532 nm, Shanghai Laser and Optics Century). Photostimulation of ChR2-expressing RTN neurons was performed using a blue laser ( $473 \mathrm{~nm}$, CrystaLaser). The lasers were controlled by TTL-pulses from a Grass model $\mathrm{S} 88$ stimulator (AstroMed Inc.). Continuous green light ( $5 \mathrm{~mW}$ ) was applied bilaterally using a splitter through $200 \mu$ m-thick multimode optical fiber (Thorlabs) in 10s episodes of continuous illumination. This method effectively silences chemosensitive RTN neurons under anesthesia (See Fig. 2 of (Basting et al., 2015)). ChR2-transduced neurons were excited with pulses of blue light ( $5 \mathrm{~ms}, \sim 9 \mathrm{~mW}$ ) delivered at $20 \mathrm{~Hz}$ for $20 \mathrm{~s}$ (Kanbar et al., 2010; Abbott et al., 2013a; Burke et al., 2014) or intermittently to entrain the breathing cycle (four 5 ms-long pulses, 50 ms interval delivered at a frequency greater than resting $f_{R}$, see (Abbott et al., 2011)).

\section{Data acquisition and analysis}

Physiological signals were acquired and processed using Spike v7.03 software (CED). EEG and EMG were amplified and band pass filtered (EEG: 0.1-100 Hz, x1000. EMG: $300-3000 \mathrm{~Hz}$, $\mathrm{x} 1000)$ and acquired at a sampling frequency of $1 \mathrm{KHz}$. The signal generated by the differential pressure transducer connected to the plethysmography chamber was amplified and band pass filtered $(0.1-15 \mathrm{~Hz}, x 100)$ and acquired at a sampling frequency of $1 \mathrm{KHz}$. The signal from the radio telemetry probe was acquired at a sampling frequency of $0.2 \mathrm{KHz}$. Mean arterial pressure 
and heart rate were extracted from pulsatile blood pressure recordings from the descending aorta based on values calibrated prior to implantation of the telemetry probe. Periods of nonREM sleep, REM sleep and quiet wake were classified on the basis of EEG, EMG activity and the patterns of cardiovascular and breathing activity. During non-REM sleep, EEG spectra were dominated by delta activity $(0.5-4 \mathrm{~Hz})$, with little or no EMG activity and a stable breathing pattern, blood pressure and heart rate. REM sleep was characterized by stable theta oscillations (6 - $8 \mathrm{~Hz}$ ), neck muscle atonia, an irregular breathing pattern and bradycardia with blood pressure fluctuations. Quiet wake was characterized by a reduction in total power with EMG tone and elevated heart rate. A minimum of 6 photoactivation trials were conducted in each rat in each state. Average breathing values were extracted from trials in non-REM sleep, REM sleep and quiet wake that were not contaminated by body movements, as indicated by EMG activity. Baseline values for all parameters were calculated from the $20 \mathrm{~s}$ preceding the photoactivation period. Respiratory frequency $\left(f_{R}\right.$, breaths $\left./ \mathrm{min}\right)$ and tidal volume $\left(V_{T}\right.$, area under the curve during the inspiratory period calibrated to waveforms generated by injecting $5 \mathrm{ml}$ of dry air from a syringe during the experiment, expressed in $\mathrm{ml}$ per 100g body weight (BW)) were calculated using Spike software v7.3 (CED). These values were used to calculate minute ventilation $\left(V_{E}=f_{R}\right.$ * $\mathrm{V}_{\mathrm{T}}$, expressed as $\left.\mathrm{ml} / 100 \mathrm{~g} \mathrm{BW} / \mathrm{min}\right)$.

All data sets were tested for normality using the Shapiro-Wilk test, then differences within and between groups were determined using one- or two-way repeated measures (RM) ANOVA with Bonferroni multiple comparisons. Linear regression, Student's t-test and nonparametric Kruskall-Wallis tests were also performed as required. All values are expressed as mean \pm SEM and significance indicated (one symbol, $p<0.05$; two symbols, $p<0.01$; three symbols, $p<0.005$, four symbols, $p<0.0001)$. 


\section{Histology}

Animals were deeply anesthetized with sodium pentobarbital and perfused transcardially with $4 \%$ paraformaldehyde, brains removed and processed as described previously (Abbott et al., 2009a; Burke et al., 2014). Immunohistochemistry with antibodies against tyrosine hydroxylase (sheep anti-TH, 1:2000, Millipore), EYFP (chicken anti-GFP, 1:1000, AVES labs) or mCherry (rabbit anti-dsRed, 1:500, Clontech \#632496, Clontech Laboratories) and Phox2b (rabbit anti-Phox2b, 1:8000, a gift from J.F. Brunet, Ecole Normale Superieure, Paris, France) were performed as previously described (Abbott et al., 2009a; Burke et al., 2014). Cell mapping, counting and photography were done using the Neurolucida system (MicroBrightfield, Inc, Colchester, VT) with a Zeiss Axioskop microscope with computer driven stage and Zeiss MRc camera. Cell counts were taken from a 1 in 6 series of $40 \mu \mathrm{m}$ sections and only profiles containing a nucleus were counted.

\subsection{Results}

\section{State-dependence of the HCVR}

Exposure to $3 \% \mathrm{FiCO}_{2}$ did not detectably perturb natural sleep in any of the rats $(\mathrm{N}=10)$. Most rats (7/10) were also able to cycle through periods of non-REM and REM sleep in the presence of $6 \% \mathrm{FiCO}_{2}$ (Fig.1).

During REM sleep, breathing was either regular or very labile (Figs. 1,4,6 and Supplemental Videos \#3-4). The average $f_{R}$ measured in REM sleep was higher than during nonREM sleep and quiet wake ( $N=10$; REM sleep: $93 \pm 3$ breaths/min; non-REM sleep: $74 \pm 3$; quiet wake: $77 \pm 4$; REM vs. non-REM, $p=0.0001$; REM vs. wake, $p=0.0003$; non-REM vs. wake, $p=$ 
0.2). $V_{T}(\mathrm{ml} /$ breath/100g) was lower in REM sleep than non-REM sleep and quiet wake $(\mathrm{N}=10$; REM sleep: $0.32 \pm 0.02$; non-REM sleep: $0.41 \pm 0.01$; quiet wake: $0.44 \pm 0.03$; REM vs. non-REM, $p=0.003 ;$ REM vs. quiet wake, $p=0.002$; non-REM vs. wake, $p=0.6)$. Minute ventilation $\left(V_{E}=f_{R}\right.$ $* \mathrm{~V}_{\mathrm{T}} ; \mathrm{ml} / \mathrm{min} / 100 \mathrm{~g}$ ) was virtually identical across the three states ( $\mathrm{N}=10 ; \mathrm{REM}$ sleep: $30 \pm 2$; non-REM sleep: $30 \pm 1$; quiet wake: $34 \pm 2$; one-way RM ANOVA: $F_{2,14}=2.969, p=0.1$ ).

Exposure to 3 and $6 \% \mathrm{FiCO}_{2}$ during non-REM and quiet wake increased $f_{R}, V_{T}$ and $V_{E}$ linearly (Fig. 1C-E). During REM sleep, $V_{T}$ also increased linearly with $\mathrm{FiCO}_{2}$ but $\mathrm{f}_{\mathrm{R}}$ was invariant.

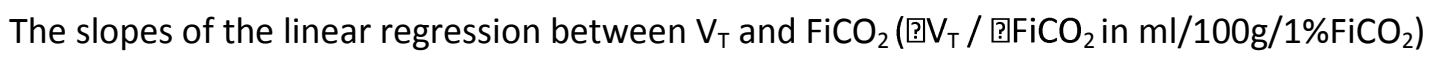
were not state-dependent (Fig. 1D; N = 7; REM sleep: $0.04 \pm 0.004$; non-REM sleep: $0.05 \pm 0.009$; wake: $0.06 \pm 0.009$; One-way RM ANOVA: $\left.F_{2,10}=3.537, p=0.07\right)$. However, during REM sleep, $f_{R}$ was unaffected by $\mathrm{CO}_{2}\left(\mathrm{~N}=7 ; 0 \% \mathrm{FiCO}_{2}: 94 \pm 3 \mathrm{bpm} ; 3 \% \mathrm{FiCO}_{2}: 94 \pm 4 ; 6 \% \mathrm{FiCO}_{2}\right.$ : $96 \pm$ 4; one-way RM-ANOVA: $\left.F_{2,12}=0.1934, p=0.8\right)$. Accordingly, the slopes of the linear regressions between $f_{R}$ and $\mathrm{FiCO}_{2}\left(\right.$ 国 $\mathrm{R}_{\mathrm{R}} /$ 黑 $\mathrm{FiCO}_{2}$ in breaths/min/1\%FiCO $)$ were markedly different (Fig. $1 \mathrm{C} ; \mathrm{N}=7 ; \mathrm{REM}$ sleep: $0.3 \pm 0.5$; non-REM sleep: $8.9 \pm 0.7$; quiet wake: $9.3 \pm 1.2$; REM vs. non-REM, $p<0.0001$; REM vs. quiet wake, $p=0.001$; non-REM sleep vs. quiet wake, $p>0.9$ ). Finally, the slopes of the regressions between $\mathrm{V}_{\mathrm{E}}$ and $\mathrm{FiCO}_{2}$ (可 $\mathrm{V}_{\mathrm{E}} /$ ? $\mathrm{FiCO}$ in $\mathrm{ml} / \mathrm{min} / 100 \mathrm{~g} / 1 \% \mathrm{FiCO}_{2}$ ) were also different during REM sleep vs. the other states (Fig. 1E; $N=7$; REM sleep: $4.2 \pm 0.6$; non-REM sleep: $9.7 \pm$ 1.2; wake: $12.3 \pm 0.6$; REM vs. non-REM, $p<0.004$; REM vs. wake, $p<0.0001$; non-REM vs. wake, $p=0.05)$.

In summary, in rats, $\mathrm{f}_{\mathrm{R}}$ is no longer under chemoreceptor control during REM sleep but the tidal volume component of the HCVR has approximately the same gain during all states.

\section{Optogenetic inhibition of RTN neurons in REM sleep reduces $V_{T}$ selectively}


Arch was photoactivated in $10 \mathrm{~s}$ episodes during established periods of non-REM sleep, REM sleep or quiet wake. During non-REM sleep and quiet wake, Arch-mediated neuronal inhibition reduced both $f_{R}$ and $V_{T}$ whereas during REM sleep Arch-activation reduced $V_{T}$ but did not change $f_{R}$ (Fig. $\left.2 A-D\right)\left(N=8 ; f_{R}\right.$ (baseline vs. Arch-activation): non-REM sleep, $p<0.0001$, REM sleep, $p>0.9$, wake, $p<0.0001 ; V_{T}$ (baseline vs. Arch-activation): non-REM sleep, $p<$ 0.0001; REM sleep, $p=0.001$, wake, $p<0.0001 ; V_{E}$ (baseline vs. Arch-activation): non-REM, $p<$ 0.0001; REM, $p=0.006$; wake, $p<0.0001)$.

We also performed these experiments under hyperoxia $\left(65 \% \mathrm{FiO}_{2}\right.$; same 8 rats), which silences the carotid bodies (Dejours, 1962; Gonzalez et al., 1994). As found previously (Basting et al., 2015), during non-REM sleep and quiet wake, Arch-activation produced a much larger reduction of $f_{R}$ and $V_{T}$ than under normoxia (group data in Fig. 2E, representative example in Fig. 3 with corresponding Supplemental Videos \#1-2). However, during REM sleep, again, Archactivation reduced $\mathrm{V}_{\mathrm{T}}$ but had no effect on $\mathrm{f}_{\mathrm{R}}$ (group data in Fig, $2 \mathrm{E}$, representative example in Fig. 4 and corresponding Videos \#3-4) ( $N=8 ; f_{R}$ (baseline vs. Arch-activation): non-REM, $p<$ 0.0001; REM, $p>0.9$; wake, $p<0.0001$. $\mathrm{V}_{\mathrm{T}}$ (baseline vs. Arch-activation): non-REM, $p=0.0001$; REM, $p=0.009 ;$ wake, $p=0.0001 . V_{\mathrm{E}}$ (baseline vs. Arch-activation): non-REM, $p<0.0001 ; \mathrm{REM}, p$ $=0.04 ;$ wake, $p<0.0001)$.

BP and HR were unaffected by photoactivation with green light (Fig. 2A-C, Table 1).

\section{RTN activation in REM sleep increases $V_{T}$ but not $f_{R}$}

ChR2-transduced neurons were photoactivated ( 5 ms pulses, $20 \mathrm{~Hz}, 20$ s episodes) during non-REM sleep, REM sleep and quiet wake. ChR2-mediated stimulation increased both $f_{R}$ and $V_{T}$ during non-REM sleep and quiet wake (Fig. 5A,C,D). In REM sleep, $V_{T}$ increased but $f_{R}$ did 
not change (Fig. 5B, D) ( $N=10 ; \mathrm{f}_{\mathrm{R}}$ (baseline vs. ChR2-stimulation): non-REM, $p<0.0001 ; \mathrm{REM}, p$ $=0.6$; quiet wake, $p<0.0001 ; \mathrm{V}_{\mathrm{T}}$ (baseline vs. ChR2-stimulation: non-REM, $p<0.0001 ; \operatorname{REM}, p=$ 0.0003; quiet wake, $p<0.0002)$.

BP and HR were unchanged by the photostimulus (Fig. 5A-C, Table 1).

\section{Phasic RTN stimulation does not entrain breathing during REM sleep and does not produce active expiration during either form of sleep}

As reported before (Abbott et al., 2011), short light trains (3-4 pulses per train, $20 \mathrm{~Hz}, 2-5 \mathrm{~ms}$ pulses) applied at frequencies above resting $\mathrm{f}_{\mathrm{R}}$ entrained the breathing cycle (range: 66-108 breaths/min; Fig. 6A). However we found that pacing could be produced during non-REM sleep and quiet wake but never during REM sleep (Fig. 6A-D).

In the absence of RTN stimulation, peak expiratory flow (PEF) occurred in early expiration (E1) when the rats were awake or in non-REM sleep. This pattern persisted during phasic RTN photostimulation when the rats were in non-REM or REM sleep (Figs. 6B,E). However, during quiet wake, the same RTN stimuli reduced E1 expiratory flow and produced a strong late expiratory flow (E2) signal indicative of active (abdominal) expiration (Fig. 6B; black arrows). This pattern of breathing is better appreciated by examining stimulus-triggered averages of the plethysmography signal (Figs. 6E, 7A-B). In 5/9 rats, a single large PEF occurred during this late-expiratory phase (Fig. 7C). The remainder of the rats displayed a biphasic expiratory flow as per (Abbott et al., 2011). We divided the expiratory phase in two segments (E1 and E2) corresponding to each of the two PEFs and measured the expiratory airflow volume occurring in each phase at rest and during phasic RTN stimulation. In quiet wake, the volume of E1 expiratory airflow at rest was $0.38 \pm 0.02 \mathrm{ml} / 100 \mathrm{~g}$ and decreased with RTN stimuli to $0.25 \pm$ 
$0.01 \mathrm{ml} / 100 \mathrm{~g}$ (Fig. 7D; N=9; two-way ANOVA with Bonferroni's multiple comparisons: Wake (rest) vs. Wake (laser), $p=0.0005)$. Conversely, E2 expiratory airflow at rest was $0.14 \pm 0.01$ $\mathrm{ml} / 100 \mathrm{~g}$ and increased with RTN stimuli to $0.25 \pm 0.02 \mathrm{ml} / 100 \mathrm{~g}(p=0.0001)$. There was no change in the relative E1 and E2 expiratory volume under resting conditions, nor with phasic RTN stimuli during non-REM sleep (Fig. 7D); total expiratory airflow (volume of E1+E2) did not differ between any groups (Fig. 7D).

In summary, phasic RTN stimulation at frequencies above resting $f_{R}$ entrained the breathing cycle. The trajectory of the expiratory flow was detectably altered only when the animals were awake. Under these circumstances, expiratory flow slowed during the early postinspiratory phase and was strongly enhanced during the late expiratory phase.

\section{During RTN-imposed pacing, selective reduction of the post-inspiratory phase accounts for the} reduction of breathing cycle length

Phasic RTN stimulation entrained breathing equally during non-REM sleep and quiet wake (Fig. 8A,B, see also Fig. 6C). As previously observed, the light stimuli always settled in the mid- to late-expiratory phase (Potts et al., 2005; Abbott et al., 2011; Pagliardini et al., 2011). During non-REM sleep, pacing shortened the expiratory phase (Fig. 8C; $\mathrm{N}=8$; Kruskal-Wallis $H=$ 41.93, $p<0.0001$ ) without changing the inspiratory duration (Fig. 8C; Kruskal-Wallis $H=7.027, p$ $=0.4)$. During wake, the post-inspiratory phase (E1) could be clearly distinguished from the active expiratory phase (E2). Pacing shortened the E1 phase (Fig. 8D. $\mathrm{N}=7$; Kruskal-Wallis $H=$ 23.76, $p=0.001$ ) without altering the E2 phase (Kruskal-Wallis $H=3.962, p=0.8$ ) nor inspiratory duration (Kruskal-Wallis $H=3.510, p=0.8$ ).

\section{Histology}


The ChR2-transduced neurons (mCherry-positive, mCherry detection enhanced by immunohistochemistry) were located under the facial motor nucleus within the rostral half of RTN (Fig. 9A,B) (Takakura et al., 2008a). The number of transduced RTN neurons (TH-negative) counted ( $1: 6$ series of coronal sections) was $32 \pm 5$ per rat $(N=8)$. These sections also contained $27 \pm 7$ catecholaminergic neurons judged to be $\mathrm{A} 5$ noradrenergic neurons rather than $\mathrm{C} 1$ adrenergic neurons based on location. RTN contains approximately 2000 neurons in rats (Takakura et al., 2008a). On average, only 10\% (range 5-18\%) were therefore transduced.

The distribution of Arch-transduced (EYFP-immunoreactive) neurons was comparable (though bilateral) and centered on the caudal rather than rostral half of RTN (Fig. 9C,D). We identified $77 \pm 15$ Arch-transduced RTN neurons (EYFP ${ }^{+} / \mathrm{TH}^{-} / \mathrm{Phox}^{2} \mathrm{~b}^{+}$) and $97 \pm 10$ catecholaminergic, probably $\mathrm{C} 1$, neurons in a one in six series of transverse sections $(N=8)$. Thus, $23 \%$ of RTN CRCs (range $20-42 \%$ ) were detectably transduced with Arch (6 x 77/2000).

\section{Green laser light control experiments}

In order to control for possible non-selective effects of continuous application of green light, the light was delivered in $10 \mathrm{~s}$ episodes bilaterally to the RTN of 5 rats that had received injections of vector but in which transduced neurons were not detected postmortem (Arch ${ }^{-}$rats; $N=5)$. The location of the optical fiber tips was recorded for both $\operatorname{Arch}^{-}(n=5)$ and $\operatorname{Arch}^{+}$rats $(\mathrm{N}=8)$. The optic fiber tips were positioned in the same region of the ventrolateral medulla oblongata in both rat groups, mostly dorsal to the RTN neurons (Fig. 10A). Light delivery during non-REM sleep or quiet wake had no effect on breathing in Arch $^{-}$rats (Fig. 10B-C). 


\subsection{Discussion}

We report two new findings. First, the respiratory drive contributed by RTN has the same state-dependency as the HCVR. Specifically, like $\mathrm{CO}_{2}, \mathrm{RTN}$ regulates $\mathrm{f}_{\mathrm{R}}$ and $\mathrm{V}_{\mathrm{T}}$ during wake and non-REM sleep whereas, during REM sleep, neither RTN nor $\mathrm{CO}_{2}$ exert any control over $\mathrm{f}_{\mathrm{R}}$. Second, RTN stimulation reduces post-inspiratory (E1) airflow and elicits active E2 expiration but this pattern is observed only during wake.

Loss of $f_{R}$ control contributes to reduced chemoreflex gain during REM sleep

As reported before (Fagenholz et al., 1976; Sullivan et al., 1979; Coote, 1982; Douglas et al., 1982a; Douglas et al., 1982b; Berthon-Jones \& Sullivan, 1984; Smith et al., 1997; Horner et al., 2002), the HCVR was similar during non-REM sleep and quiet wake but significantly reduced during REM sleep. We also confirm that hypercapnia does not change $f_{R}$ during REM sleep (Berthon-Jones \& Sullivan, 1984; Horner et al., 2002; Nakamura et al., 2007). By contrast, hypercapnia reportedly produces comparable increases in diaphragmatic $E M G$ and $V_{T}$ across wake, non-REM and REM sleep states (Haxhiu et al., 1987; Horner et al., 2002; Nakamura et al., 2007). Consistent with these observations, a given increment of $\mathrm{FiCO}_{2}$ produced a similar $\mathrm{V}_{\mathrm{T}}$ increase during wake, non-REM sleep and REM sleep in our experiments.

In short, the HCVR persists during REM sleep but with reduced gain because this reflex operates only via changes in $\mathrm{V}_{\mathrm{T}}$. This characteristic seems present in all mammals including man. Its impact on the HCVR gain is presumably larger in species like rodents in which $f_{R}$ stimulation contributes most to the reflex. As is the case during REM sleep, homeostatic control of $\mathrm{PCO}_{2}$ via changes in $V_{T}$ persists during voluntary breathing (Haouzi \& Bell, 2009; Ohashi et al., 2013). 
Inability of RTN to regulate $f_{R}$ contributes to reduced chemoreflex during REM sleep

In rats, the breathing pattern in REM sleep alternates between periods during which $f_{R}$ is increased but remains fairly regular and periods of marked breathing lability. In neither phase could we modify $f_{R}$ by stimulating or inhibiting RTN. Thus the inability of RTN neurons to control $f_{R}$ underlies, partly at least, the loss of chemoreceptor control over $f_{R}$ during REM sleep. Our observations also rule out the hypothesis that RTN could be mediating the tachypnea occurring during REM sleep (Fraigne \& Orem, 2011).

Two possibilities could explain RTN's inability to regulate $f_{R}$ during REM sleep: (a) RTN could be silent or unexcitable or (b) this nucleus could remain active and $\mathrm{CO}_{2}$-responsive but its effect on the rhythm generator could be gated out downstream. Although RTN neurons are mildly activated by serotonin, a transmitter whose release presumably decreases during REM sleep in this brain region as elsewhere (Veasey et al., 1995; Mulkey et al., 2007), the first interpretation (a) is the least plausible. First, optogenetic activation of RTN or hypercapnia still increased $V_{T}$ during REM sleep indicating that RTN does remain excitable. Even if distinct subtypes of RTN neurons regulated $f_{R} v s . V_{T}$ it seems unlikely that only the former would become totally unresponsive to ChR2 activation during REM sleep. Second, optogenetic inhibition of RTN inhibited $V_{T}$ similarly during wake, non-REM and REM sleep indicating that these neurons remain active at rest during REM sleep. Accordingly, a more plausible explanation is that, during REM sleep, neither RTN nor $\mathrm{CO}_{2}$ controls $\mathrm{f}_{\mathrm{R}}$ because the respiratory rhythm generator is unresponsive to chemoreceptor input.

State-dependent control of active expiration by RTN 
Active expiration (Janczewski \& Feldman, 2006; Feldman et al., 2013), can be elicited by activating RTN and surrounding CA neurons in conscious rats ((Abbott et al., 2011) and present results). In the in situ working heart-brainstem preparation in which lumbar expiratory activity persists at rest, this outflow is eliminated by inhibiting the same neuronal mix (Marina et al., 2010). The present study adds three novel elements. First, active expiration is elicited by stimulating the rostral portion of RTN which contains hypercapnia-activated neurons but no C1 cells (Takakura et al., 2008a). Thus, C1 cell stimulation is not required to produce active expiration. Second, RTN stimulation causes active expiration only when the rats are awake. Third, we suggest that upper airway resistance increases in parallel with active expiration, as denoted by the significant reduction in early expiratory (E1) airflow and the proportional increase in late expiratory (E2) airflow. In sum, we show that, along with active expiration, RTN stimulation produces a brief wake state-dependent facilitation of glottis closure immediately after inspiration to maintain expiratory lung volume, presumably for increased gas exchange. Thus, RTN stimulation appears capable of increasing alveolar ventilation via at least four mechanisms: Increased inspiratory tidal volume, increased $f_{R}$, brief retention of inspired air during the early expiratory phase and active expiration. According to the present results, the latter two mechanisms operate only during the waking state. However, we do not exclude the possibility that active expiration could be triggered even during non-REM sleep by stimulating a larger fraction of RTN neurons than in the present study.

Components of the circuitry responsible for active expiration (expiratory rhythm generator) overlap anatomically with the caudal RTN (Pagliardini et al., 2011; Feldman et al., 2013; Tupal et al., 2014). As shown here, active expiration can be triggered by stimulating RTN neurons located rostral to this region (Pagliardini et al., 2011; Feldman et al., 2013). Thus RTN, 
as defined in this laboratory (Phox $2 \mathrm{~b}^{+} / \mathrm{VGLUT2}^{+} / \mathrm{NK} \mathrm{R}^{+}$neurons located ventral to the facial motor nucleus)(Guyenet \& Mulkey, 2010), can activate this oscillator but is probably not part of it. Consistent with this interpretation, RTN stimulation elicits active expiration only during wake whereas the same neurons increase $f_{R}$ and inspiratory amplitude equally during non-REM sleep and quiet wake; the same reasoning applies for the effects of RTN on post-inspiratory airflow. The fact that RTN can only facilitate active expiration or laryngeal adduction during wake signifies that, in absence of exercise, the recruitment of these muscles for breathing requires both a high level of CRC activation and a heightened network excitability presumably conferred by wake-ON neuromodulators (e.g. serotonin, noradrenaline, orexin) (Doi \& Ramirez, 2008).

RTN innervates all the RPG regions that harbor excitatory pump premotor neurons (Ballantyne \& Richter, 1986; Dobbins \& Feldman, 1994; Yokota et al., 2007; Bochorishvili et al., 2012). The excitatory input from RTN plausibly increases $V_{T}$ by enhancing the discharges of these premotor cells. Phrenic motor neurons remain active regardless of the state of vigilance hence the relative state-independence of the control of inspiratory amplitude by RTN and other chemoreceptors. By contrast, the activity of lumbar and other expiratory pump muscles is highly state-dependent, like that of other postural muscles or the musculature regulating upper airway resistance. This characteristic likely explains why RTN stimulates active expiration only during wake. Reductions of the activity of serotonin, noradrenaline and orexin neurons likely contribute to the reduced excitability of expiratory motor neurons or their cognate premotor inputs during sleep (Doi \& Ramirez, 2008; Horner, 2009; Saper et al., 2010).

Expiratory airflow is regulated by upper airway resistance. The reduced E1 airflow with phasic RTN stimulation is likely a facilitation of glottic adduction by laryngeal constrictor (LC) 
muscles. The post-inspiratory activity of LC motor neurons could be enhanced by polysynaptic drive via the Kolliker Fuse, a major target of RTN (Dutschmann \& Herbert, 2006; Bochorishvili et al., 2012), and/or a network effect that augments phasic inspiratory inhibition of LC motor neurons and strengthens their post-inhibitory rebound (Sun et al., 2008; Bautista et al., 2010).

\section{RTN stimulation selectively shortens the post-inspiratory phase}

During quiet wake, phasic RTN stimulation increased $f_{R}$ strictly by shortening the postinspiratory (E1) phase. In vitro, somatic afferent stimulation also produces tachypnea in this manner (Potts et al., 2005). In this case, the tachypnea is attributed to disinhibition of the rhythmogenic pre-inspiratory/inspiratory (prel/I) neurons of the pre-Bötzinger complex as follows (Potts et al., 2005; Smith et al., 2013): activation of inhibitory E2 Bötzinger neurons by somatic afferents would cause early termination of E1 inhibitory neurons and, consequently, earlier depolarization of the prel/I neurons (Potts et al., 2005). RTN neurons could produce tachypnea via this mechanism because they are excitatory and innervate the Bötzinger region (Mulkey et al., 2004; Bochorishvili et al., 2012). However, RTN neurons also project heavily to the preBötzinger complex, including to NK1-receptor expressing neurons that are suspected to include the prel/I cells (Gray et al., 1999; Bochorishvili et al., 2012). An excitatory input from RTN to these cells should also theoretically be able to reduce the duration of their inhibition during E1, allowing their burst discharges to occur earlier and $f_{R}$ to rise.

\section{Why is RTN no longer controlling $f_{R}$ during REM sleep?}

The transition from an auto-rhythmic homeostatically regulated state (anesthesia, reduced preparations, non-REM sleep and quiet wake) to other forms of breathing (voluntary, emotional, REM sleep) is poorly understood. However, in general terms, the "commanded" 
model, where inspiration is still triggered by the pontomedullary RPG, but its timing is tightly controlled by powerful inhibitory inputs from higher source(s) (Richter \& Smith, 2014) seems the most plausible for several reasons. The pontomedullary RPG is clearly engaged during both REM sleep and volitional control (Lovering et al., 2006) and the integrity of the pre-Bötzinger circuitry is required for normal breathing during both REM and non-REM sleep (McKay \& Feldman, 2008). Finally, given that RTN projections are propriobulbar (Bochorishvili et al., 2012), the homeostatic control of $\mathrm{V}_{\mathrm{T}}$ during REM sleep must occur via the ponto-medullary RPG.

Post-inspiratory inhibition is probably far more critical to the voluntary control of breathing than to breathing automaticity during quiet wake and non-REM sleep (Guz, 1997; Richter \& Smith, 2014). The irregular breathing pattern present during REM sleep may share this characteristic. A plausible reason why RTN no longer controls $f_{R}$ during REM sleep could be that the excitatory effect of RTN on the prel/I neurons is gated out during REM sleep by powerful inhibitory inputs from outside the pontomedullary RPG which clamp the membrane potential of these neurons during the expiratory phase of the breathing cycle (Richter \& Smith, 2014).

\section{Physiological and pathophysiological relevance}

The present observations could explain why many forms of CSA including CCHS occur predominantly during non-REM sleep (Berssenbrugge et al., 1983; Farney et al., 2003; Lovering et al., 2012). During REM sleep, $V_{E}$ depends more on $f_{R}$, which rises while $V_{T}$ decreases. Because $f_{R}$ is no longer under chemoreceptor control during REM sleep, $V_{E}$ is less dependent on $\mathrm{PaCO}_{2}$ than during non-REM sleep. Therefore recurrent cycles of hyperventilation and apneas presumably cannot be sustained during REM sleep because, as shown here in rats, CRCs such as RTN contribute much less to ventilation. 


\section{Experimental limitations}

PRSx8-based lentiviral vectors transduce a mixed population of RTN and catecholaminergic neurons as described before (Abbott et al., 2009a; Marina et al., 2010;

Abbott et al., 2011). In the ChR2 experiments, most transduced neurons (54\%) were TH-negative hence putative CRCs. These neurons were located within the rostral half of RTN which contains A5 noradrenergic neurons but few C1 adrenergic cells (Takakura et al., 2008a). Selective C1 cell stimulation raises BP massively in conscious rats (Burke et al., 2014). Here BP was unaffected. Thus few C1 neurons could have been directly or synaptically activated and, consequently, these neurons could not have produced the observed breathing stimulation. Some contribution of A5 neuron stimulation to the breathing effects observed presently cannot be excluded.

For the loss of function experiments (Arch experiments), we needed to target the caudal RTN in order to transduce more CRCs. This region contains numerous C1 cells and, consequently, $56 \%$ of the Arch-transduced neurons were TH-ir. Under anesthesia, Arch activation effectively silenced RTN neurons (Basting et al., 2015). In anesthetized rats, sympathetic tone is high, the $\mathrm{C} 1$ cells are very active and their inhibition produces profound hypotension consistent with their sympathoexcitatory function (Schreihofer \& Guyenet, 1997; Burke et al., 2008; Marina et al., 2011; Guyenet et al., 2013b). Here, in conscious rats, Arch photostimulation had no effect on BP. One explanation is that sympathetic tone is low in resting unstressed and normoxic animals and the transduced C1 cells have a correspondingly low resting discharge. Another possibility is that the Arch-transduced C1 cells were inadequately hyperpolarized by the light. In any event, the lack of BP change indicates that C1 cell inhibition 
was minor at best. Consequently, a change in the activity of the $\mathrm{C} 1$ cells is very unlikely to have contributed significantly to the observed respiratory changes.

Summary and conclusions

RTN regulates both $f_{R}$ and $V_{T}$ when the pontomedullary respiratory pattern generator is auto-rhythmic (anesthesia, non-REM sleep, quiet wake) but RTN no longer controls $f_{R}$ during REM sleep. We speculate that during REM sleep, as during voluntary breathing, the expiratory phase of the breathing cycle is controlled by suprabulbar inputs that powerfully inhibit the preBötzinger complex and shunt out the excitatory effect of RTN on the rhythmogenic core. A higher resting $f_{R}$ that is no longer subject to control by RTN could explain the absence of periodic breathing and central sleep apnea during REM sleep in man. 
Table 1: Blood pressure and heart rate at rest or during Arch or ChR2 photostimulation

\begin{tabular}{|l|c|c|c|c|c|c|}
\hline State & \multicolumn{2}{|c|}{ Non-REM sleep } & \multicolumn{2}{c|}{ REM sleep } & \multicolumn{2}{c|}{ Quiet wake } \\
\hline Variable & Rest & Arch & Rest & Arch & Rest & Arch \\
\hline N & 5 & 5 & 4 & 4 & 5 & 5 \\
\hline MAP (mmHg) & $109 \pm 4$ & $108 \pm 4$ & $119 \pm 2$ & $117 \pm 2$ & $115 \pm 6$ & $113 \pm 5$ \\
\hline HR (bpm) & $300 \pm 9$ & $300 \pm 9$ & $289 \pm 6$ & $287 \pm 9$ & $310 \pm 8$ & $311 \pm 8$ \\
\hline & & & & & & \\
\hline Variable & Rest & ChR2 & Rest & ChR2 & Rest & ChR2 \\
\hline N & 7 & 7 & 7 & 7 & 7 & 7 \\
\hline MAP (mmHg) & $109 \pm 3$ & $106 \pm 3$ & $114 \pm 4$ & $113 \pm 4$ & $109 \pm 4$ & $108 \pm 3$ \\
\hline HR (bpm) & $306 \pm 7$ & $315 \pm 8$ & $298 \pm 6$ & $304 \pm 5$ & $314 \pm 12$ & $313 \pm 12$ \\
\hline
\end{tabular}

Telemetric blood pressure and heart rate recordings in rats habituated to the plethysmography chamber during various states of vigilance. No change in resting mean arterial blood pressure (MAP) or heart rate (HR) was observed during photostimulation of $\mathrm{Arch}^{+}$or $\mathrm{ChR2}^{+}$rats Statistics: 2-way ANOVA. 


\subsection{Figures}

\section{Figure 1.}
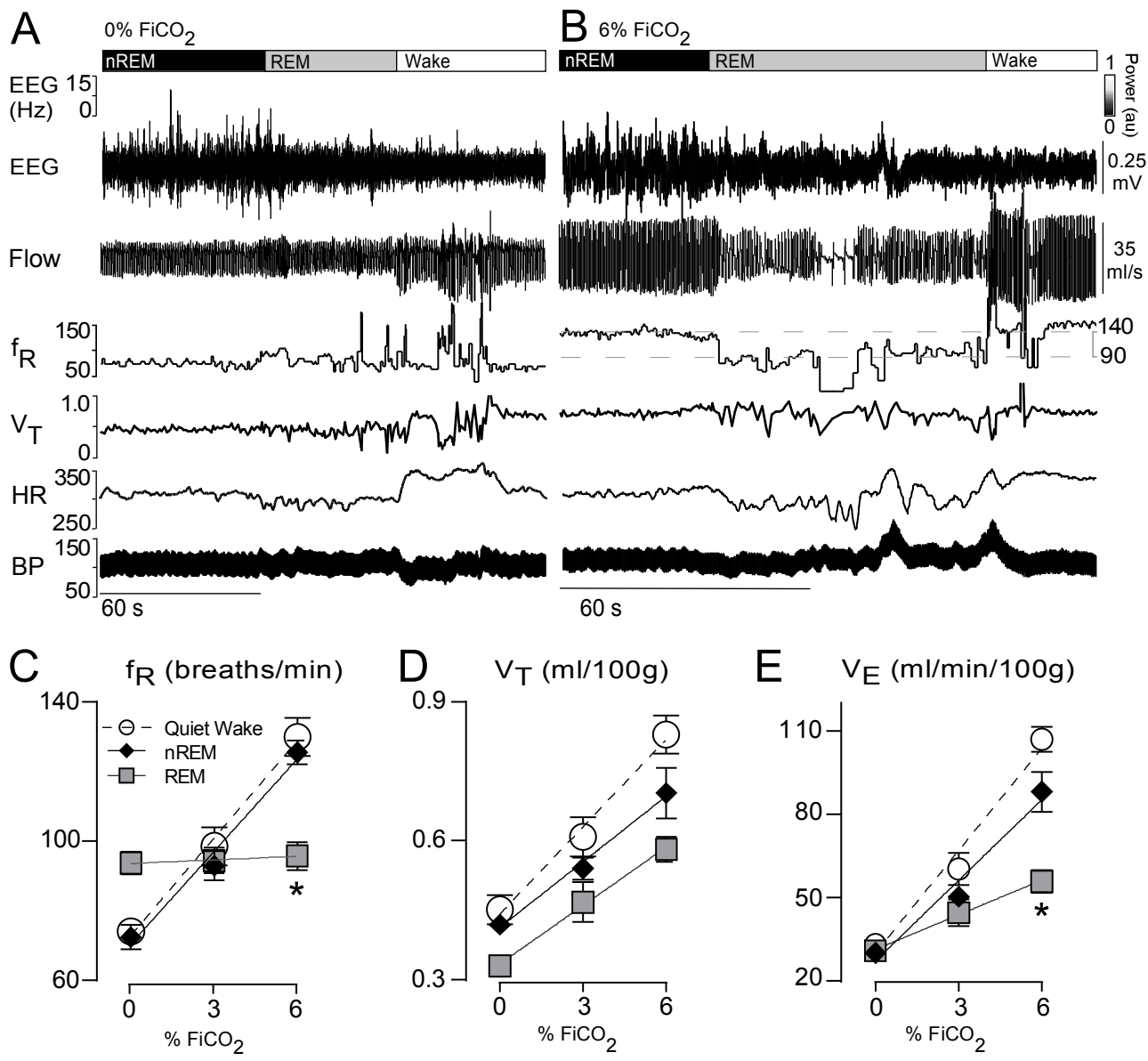


\section{Figure 1: State-dependence of the hypercapnic ventilatory reflex in rat}

A. Breathing and cardiovascular function at rest. Shown from top to bottom: EEG power spectrum (0-15 Hz), EEG (raw signal), air flow signal (plethysmography, inspiration downward), $f_{R}$ (breaths/minute), $V_{T}(\mathrm{ml} / 100 \mathrm{~g}), \mathrm{HR}$ (beats/minute) and BP (telemetric recording, $\mathrm{mmHg}$ ). During non-REM sleep, EEG power was concentrated within the delta range (0.5-4 Hz), REM sleep was identified by a strong concentration of power in the $6-8 \mathrm{~Hz}$ range (theta) and quiet wake by a large reduction in total power (desynchronized state). Breathing during REM sleep was faster, more labile and shallower than during non-REM sleep. Bradycardia and BP fluctuations also commonly occurred in REM sleep, the result of co-activation of cardiac vagal and sympathetic vasomotor outflows by central command with impaired baroreflex function. B. Breathing and cardiovascular function in hypercapnia (6\% $\mathrm{FiCO}_{2}$; same rat as in $\left.(A)\right) . V_{T}$ and $f_{R}$ were markedly increased in non-REM sleep or wake. Transition from non-REM sleep into REM sleep caused an abrupt reduction in breathing rate that was reversed at the transition to wake (hatched horizontal lines denote the $\sim 50$ breaths/min reduction in $f_{R}$ during REM sleep in this example). No differences in BP and HR regulation were observed under hypercapnia. C-E. $f_{R}, V_{T}$ and $\mathrm{V}_{\mathrm{E}}$ at $0 \%, 3 \%$ and $6 \% \mathrm{FiCO}_{2}$ measured across sleep-wake states $(\mathrm{N}=7) .{ }^{*}$ gain (slope of linear regressions) significantly different during REM vs. both quiet wake $(p=0.0001)$ and REM sleep ( $p=0.004$; see Results section for detailed statistics). 
Figure 2.

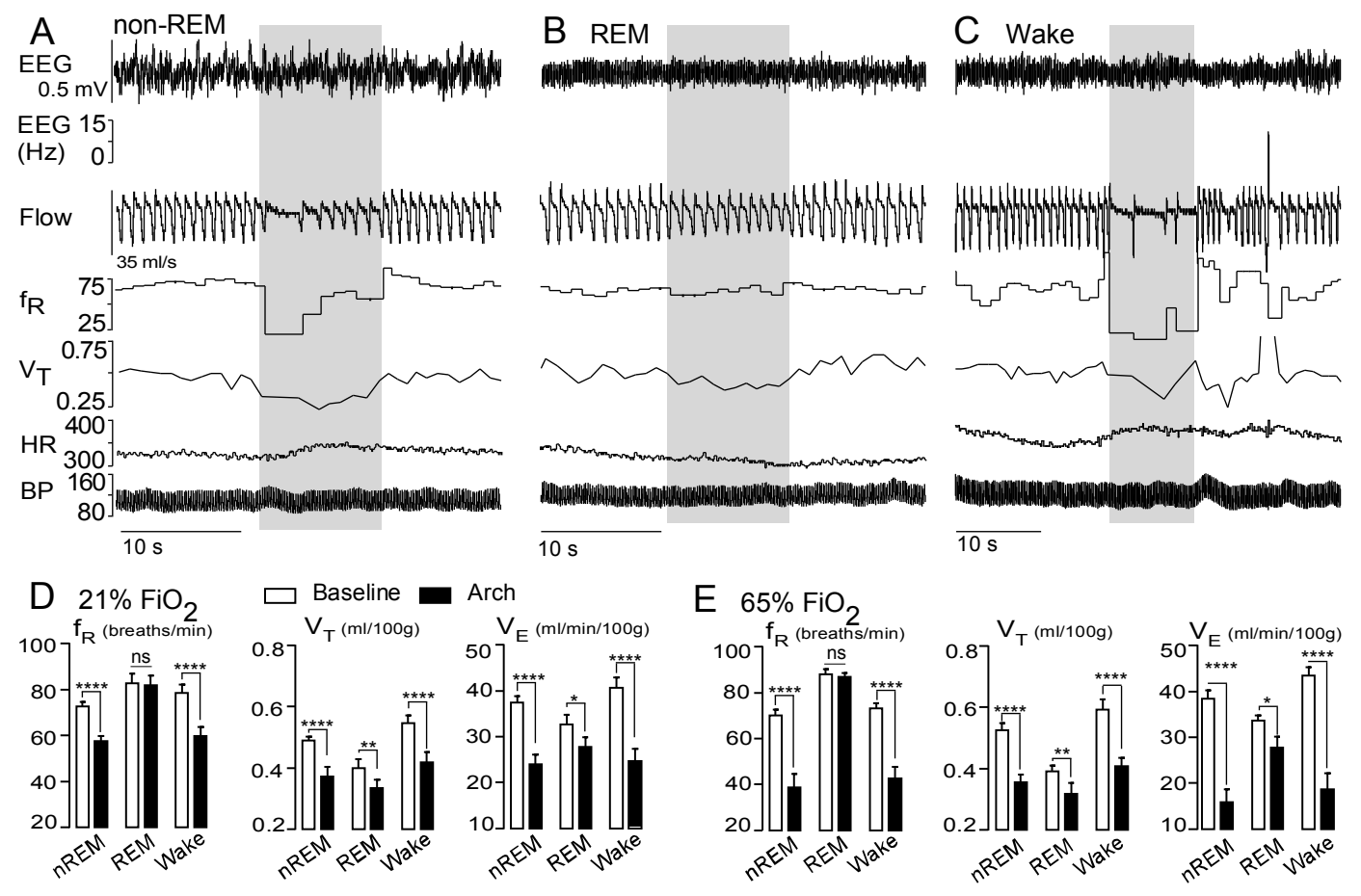


Figure 2: State-dependence of the breathing changes evoked by RTN inhibition

A-C. Hypoventilation caused by bilateral Arch-activation (10 s) in one rat at $21 \% \mathrm{FiO}_{2}$ in $(\mathrm{A})$ nonREM sleep, (B) REM sleep and (C) quiet wake. Arch-activation decreased $\mathrm{V}_{\mathrm{T}}$ in all three states, decreased $f_{R}$ during non-REM sleep and quiet wake but did not change $f_{R}$ during REM sleep. Arch-activation did not change BP, HR or sleep state. D. Ventilation parameters at rest and during Arch-activation in normoxia $\left(21 \% \mathrm{FiO}_{2} ; \mathrm{N}=8\right)$. E. Ventilation parameters at rest and during Arch-activation in hyperoxia $\left(65 \% \mathrm{FiO}_{2} ; \mathrm{N}=8\right)$. The effects of Arch-activation on ventilation were amplified in hyperoxia but qualitatively the same as in normoxia. In REM sleep, Arch-activation had no effect on $f_{R}$. Arch activation reduced $V_{T}$ under all conditions. Refer to online data supplement for video examples of Arch-activation in hyperoxia. Significance (baseline vs. Arch stimulation; two-way RM ANOVA with Bonferroni's correction for multiple comparisons): ${ }^{*} p<0.05,{ }^{* *} p<0.01,{ }^{* * *} p<0.005,{ }^{* * *} p<0.001$. 
Figure 3.

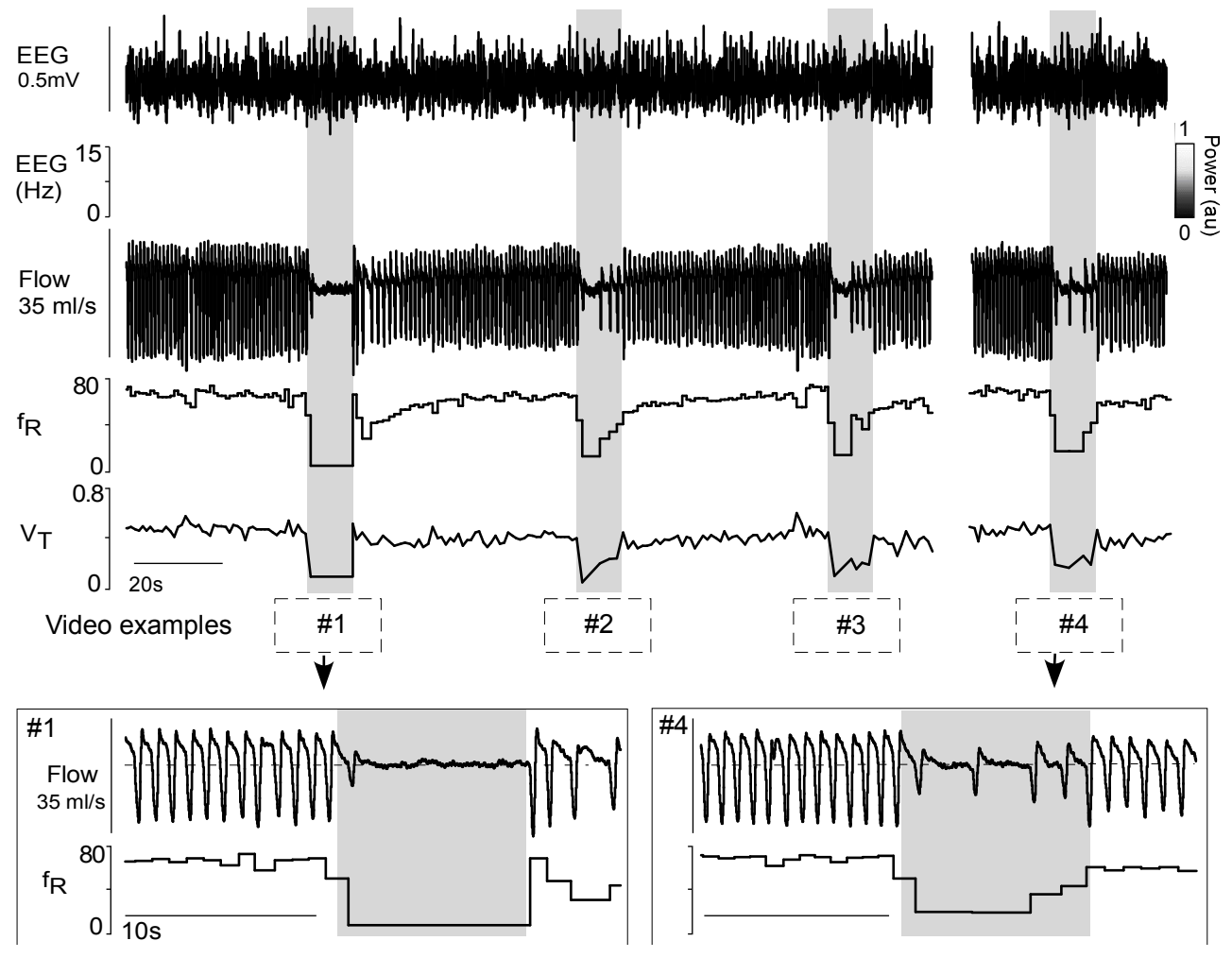




\section{Figure 3: RTN inhibition during non-REM sleep in hyperoxia}

Profound hypoventilation caused by bilateral Arch-activation (10 s; grey rectangles) in one rat at $65 \% \mathrm{FiO}_{2}$ in non-REM sleep. Despite this sudden hypoventilation, the animal does not arouse from non-REM sleep (EEG delta wave activity: $0.5-4 \mathrm{~Hz}$ ). Traces correspond to the four examples shown in Supplemental videos \#1-2. Bottom panels: enlarged traces of trials \#1 and \#4. 
Figure 4.

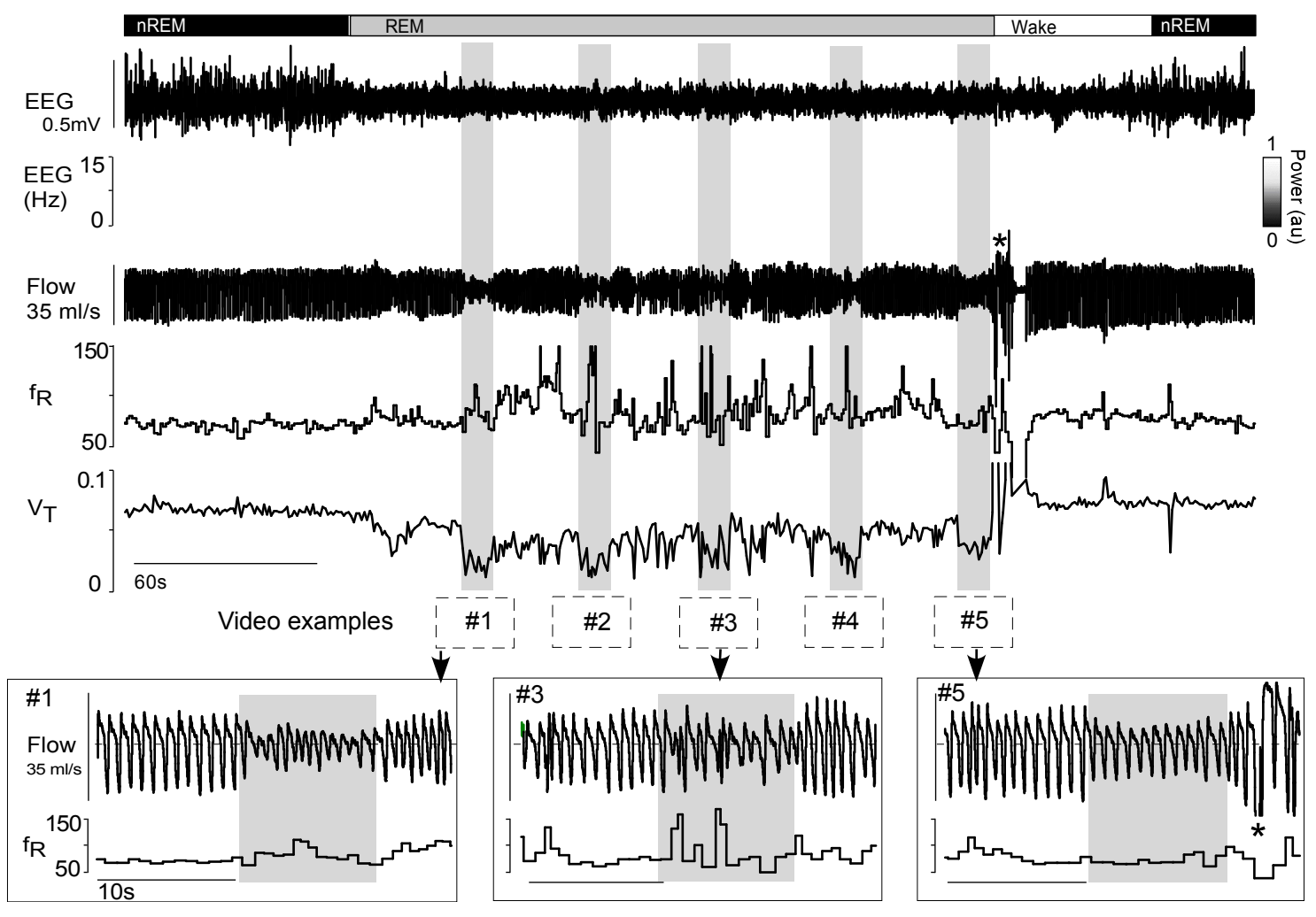




\section{Figure 4: RTN inhibition during REM sleep in hyperoxia}

Bilateral Arch-activation (10 s; grey rectangles) in one rat at $65 \% \mathrm{FiO}_{2}$ in $\mathrm{REM}$ sleep decreased $\mathrm{V}_{\mathrm{T}}$ in all trials, but did not reduce $f_{R}$ (same animal as Fig. 3). Traces correspond to the five examples in the Supplemental videos \#3-4. Bottom 3 panels: enlarged traces of trials \#1, \#3 and \#5. Animal awoke from REM sleep 3s after the fifth trial (asterisk) then returned to non-REM sleep. 


\section{Figure 5.}

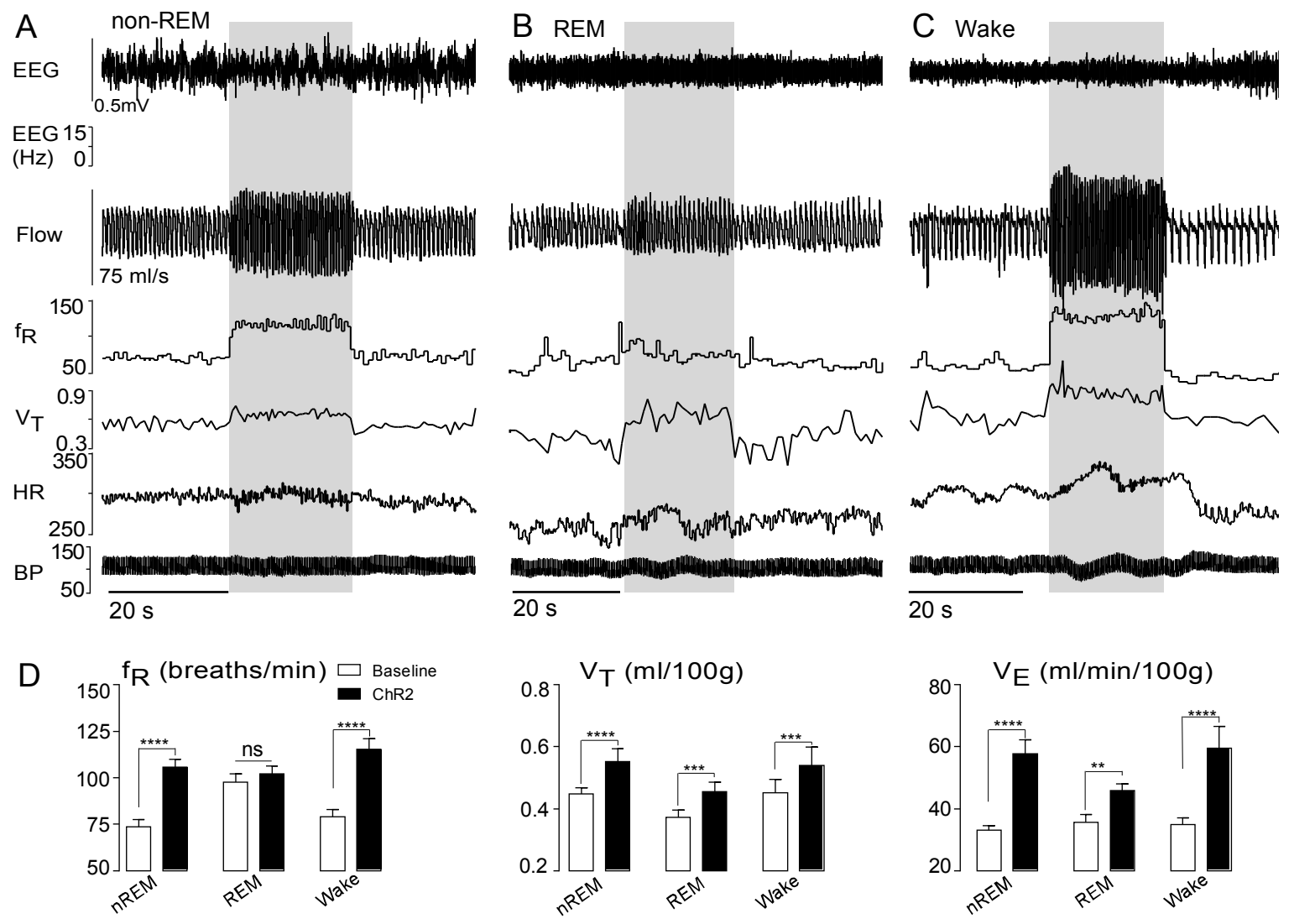


Figure 5: State-dependence of the breathing changes evoked by rostral RTN stimulation

A-C. Hyperventilation evoked by unilateral ChR2-activation ( $20 \mathrm{~s}$, blue bar) in one rat at $21 \%$ $\mathrm{FiO}_{2}$ in (A) non-REM sleep, (B) REM sleep and (C) quiet wake. ChR2-activation increased $\mathrm{V}_{\mathrm{T}}$ in all three states, increased $f_{R}$ in non-REM and quiet wake states but had no effect on $f_{R}$ during REM sleep. ChR2-activation had little effect on BP and did not produce arousal from sleep. D. Ventilation parameters at rest and during ChR2-activation in rats across sleep-wake states in normoxia $(N=10)$. Significance (baseline vs. Arch stimulation; two-way RM ANOVA with Bonferroni's correction for multiple comparisons): ${ }^{* *} p<0.01,{ }^{* * *} p<0.005,{ }^{* * * *} p<0.001$. 
Figure 6.

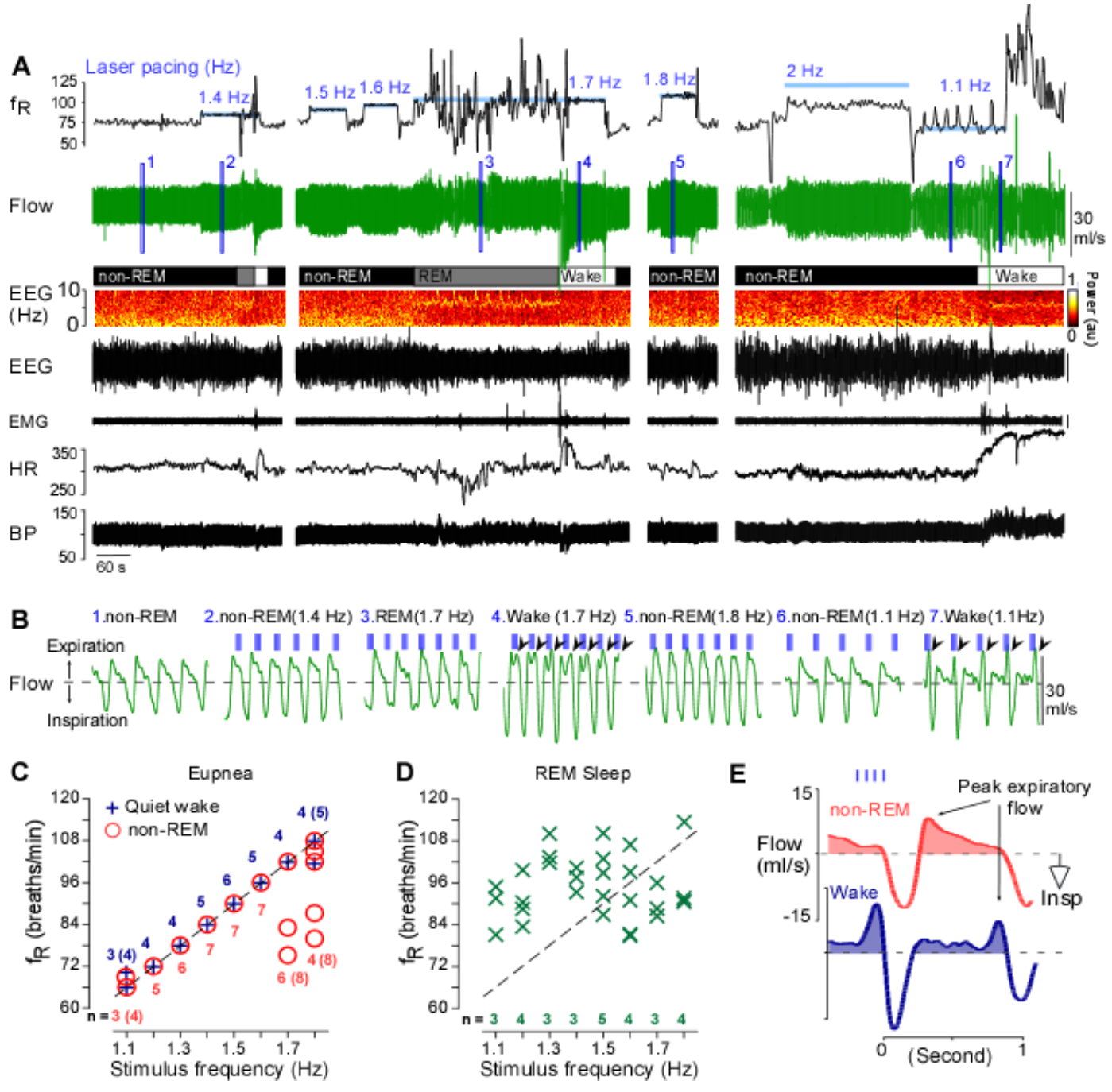




\section{Figure 6: Breathing entrainment by RTN stimulation is state-dependent.}

A. Example of breathing entrainment elicited by photostimulation of RTN (trains of $4 \times 3 \mathrm{~ms}$ light pulses at $20 \mathrm{~Hz}$; stimulation at blue bars in $\mathrm{f}_{R}$ trace). Faithful entrainment ( $f_{R}=$ train frequency) in the range of $66-108$ breaths/min $(1.1-1.8 \mathrm{~Hz})$ was obtained during non-REM sleep and wake but entrainment failed at $2 \mathrm{~Hz}$. During REM sleep, no entrainment was produced. B. Expanded plethysmography flow signals (light pulses delivered at vertical blue lines above flow traces; excerpts 1-7 taken from where indicated in the flow trace of panel A). During entrainment, the light pulses "settled" spontaneously during late- to mid-expiration. Note that during non-REM sleep peak expiratory flow at rest (\#1) or during entrainment (\#2, \#5-6) occurs in early expiration. However, in wake, RTN stimulation produced a switch in peak expiratory flow to the late-expiratory phase (arrowheads in excerpts 4 and 7), indicative of active expiration (Abbott et al., 2011; Pagliardini et al., 2011). C. Group data from 11 animals showing the relationship between stimulus train frequency and $f_{R}$ during non-REM sleep and wake states. Breathing was faithfully entrained between $1.2-1.6 \mathrm{~Hz}$ in all animals (single symbol for all animals); incomplete or unsuccessful entrainment outside this range is indicated by the individual symbols. Adjacent numbers are the number of animals that were successfully entrained at a given stimulation frequency (bracketed numbers indicate total rat number, including unsuccessful trials). Not every frequency was tested in every animal in each sleep-wake state; hence, the varying number of determinations for each tested frequency. D. Group data during REM sleep (same rats as in C). There was no relationship between the stimulus train frequency and $f_{R}$. E. Laser lighttriggered waveform averages of the respiratory flow signal during entrainment at $1.1 \mathrm{~Hz}$ (light pulses in sky blue) in non-REM sleep (red) and wake (deep blue) with the shaded regions 
denoting the expiratory flow. In this example, RTN stimulation switched peak expiratory flow (PEF) from early expiration in non-REM sleep to late-expiration (active expiration) in wake. 
Figure 7.
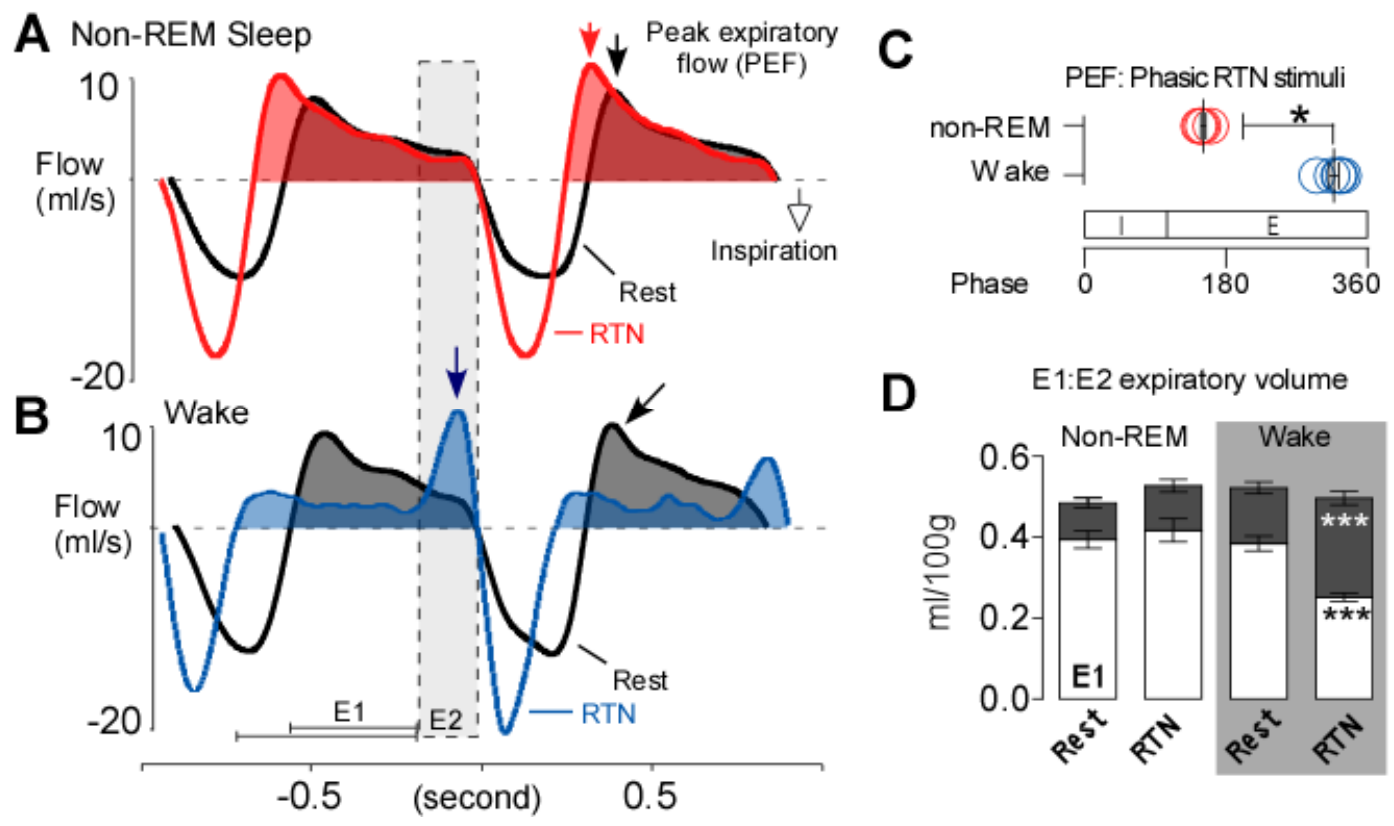
Figure 7: Wake state-dependent control of active expiration and upper airways resistance by the RTN

A-B. Event-triggered waveform averages of the plethysmography signal at rest (black trace) and during phasic RTN stimulation (colour trace) in one rat during non-REM sleep (A) or quiet wake (B). Arrows point to peak expiratory airflow (PEF). In non-REM sleep (panel A) PEF occurred in early expiration (E1) regardless of whether RTN was activated or not . In the wake state (panel B) PEF shifted from early to late expiration (E2; active expiration) during RTN stimulation and early expiratory flow (E1 phase) was markedly reduced, presumably due to increased upper airway resistance. C. Relationship between peak expiratory flow and the respiratory cycle with RTN stimulation during non-REM and quiet wake states. I. Inspiration, E expiration. N = 5. MannWhitney non-parametric test. ${ }^{*} p=0.02$. D. Expiratory airflow during early (E1; unfilled) and late (E2; gray fill) expiratory phases at rest or during phasic RTN stimuli in non-REM sleep or quiet wake. $\mathrm{N}=9$. Two-way ANOVA with Bonferroni's multiple comparison test. ${ }^{* * *} p<0.0005$. 
Figure 8.
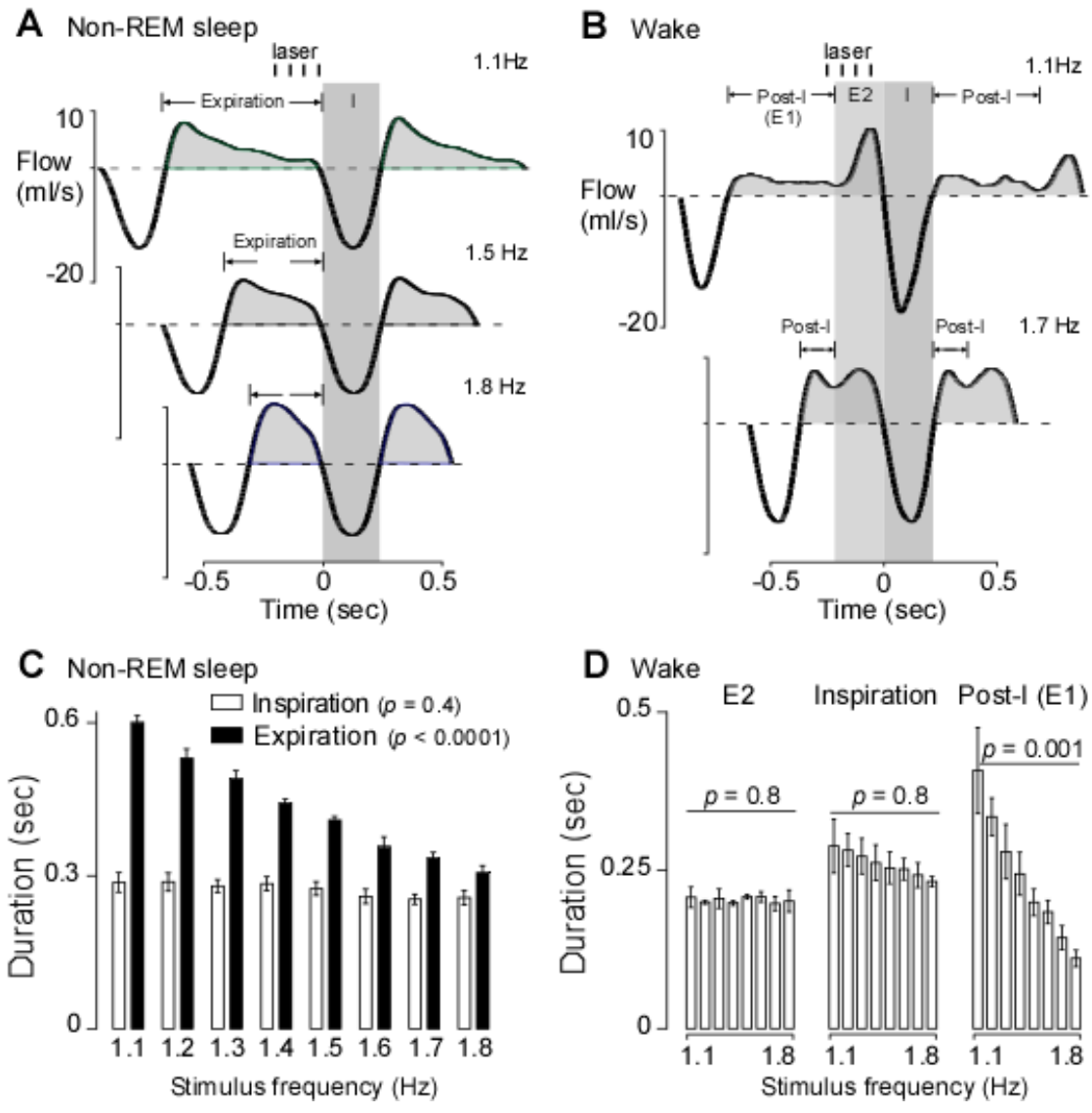
Figure 8: Phasic RTN stimulation selectively shortens the post-inspiratory phase

A-B. Event-triggered waveform averages of the plethysmography signal during light-induced breathing entrainment in one rat (A) during non-REM sleep and (B) during quiet wake. ChR2transduced RTN neurons were photostimulated with trains of $4 \times 3$ ms-long light pulses $(20 \mathrm{~Hz}$; represented as vertical blue bars) delivered at various frequencies between 1.1-18 Hz. Active expiration (large flow signal immediately preceding inspiration) was observed only during quiet wake periods. During non-REM sleep we measured only the I and E phases. During quiet wake, the duration of the late expiratory flow peak was defined as the E2 phase and the postinspiratory phase (E1) represents the balance of the expiratory duration. C-D. Group data from eight animals. Effect of stimulation frequency on the various phases of the breathing cycle evaluated using unpaired, non-parametric One-way ANOVA (Kruskall Wallis). 
Figure 9.
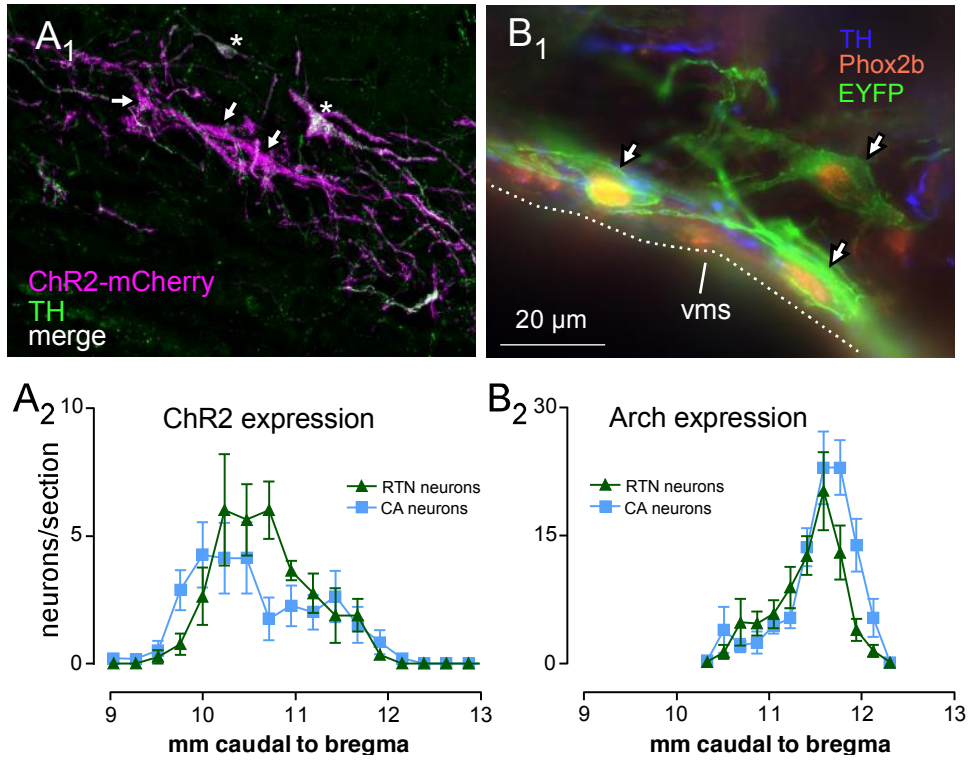
Figure 9: Location and phenotype of ChR2- or Arch-transduced neurons.

A1: Cluster of ChR2-transduced neurons within the rostral RTN (transverse plane; approximate location: $10.2 \mathrm{~mm}$ caudal to bregma). Three out of 5 neurons were not TH-ir (arrows) and were presumptive RTN CRCs, the other two (stars) were TH-ir (A5 noradrenergic neurons). A2: rostrocaudal distribution of ChR2-transduced RTN and catecholaminergic neurons $(\mathrm{N}=8)$. Neurons were transduced on the left side of the brain only.

B1: Three Arch-transduced putative RTN CRC neurons (arrows) located next to the ventral medullary surface (VMS; transverse section at $11.4 \mathrm{~mm}$ caudal to Bregma; green: Arch-EYFP; blue: TH; red: Phox2b). B2: rostrocaudal distribution of Arch-EYFP-transduced neurons ( $\mathrm{N}=8$; RTN neurons $=\mathrm{TH}^{-} /$Phox $2 \mathrm{~b}^{+} /$EYFP; CA neurons: $\left.\mathrm{TH}^{+} / \mathrm{Phox}^{2} \mathrm{~b}^{+} / \mathrm{EYFP}^{+}\right)$. Neurons were transduced bilaterally. Distance from bregma according to a standard rat atlas (Paxinos \& Watson, 2005) (sections aligned using caudal end of facial motor nucleus as level $11.6 \mathrm{~mm}$ caudal to bregma). Calibration bar in B1 equals $100 \mu \mathrm{m}$ (A1) or $20 \mu \mathrm{m}$ (B1). 
Figure 10.
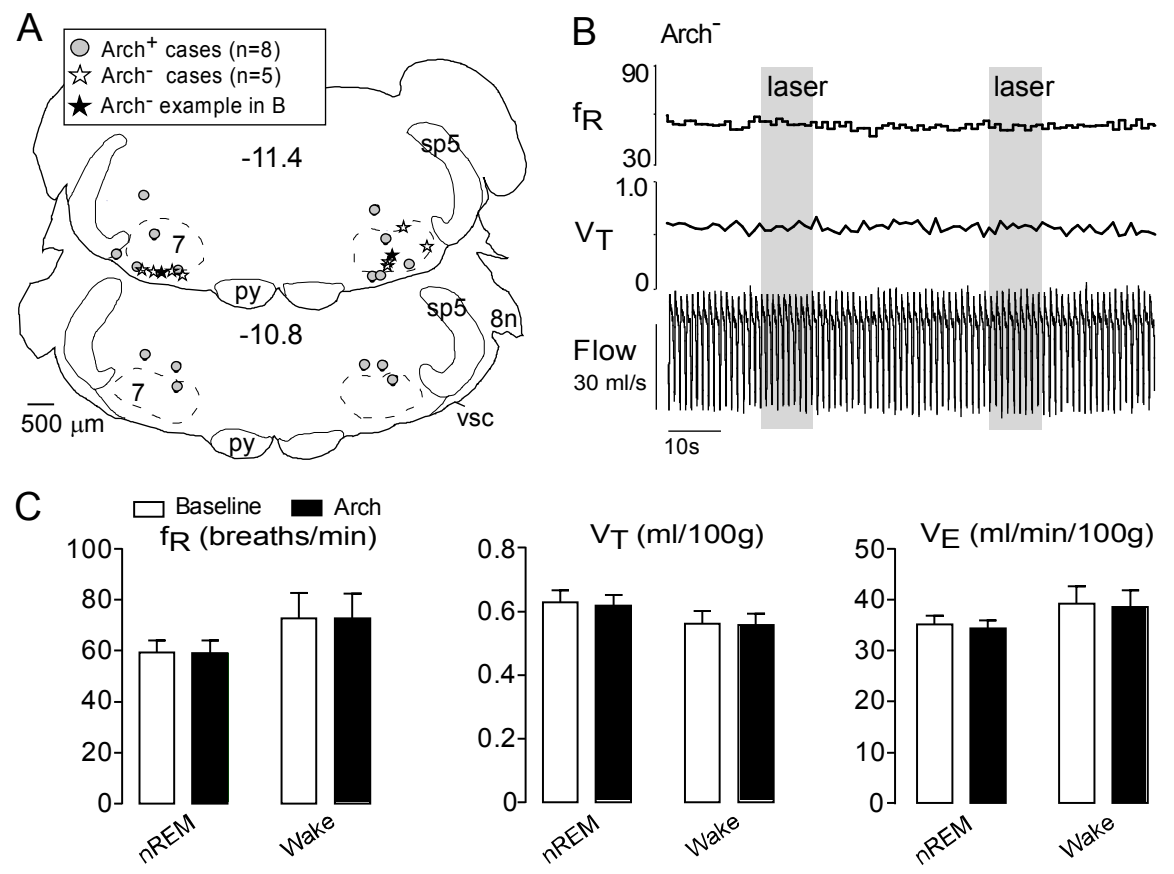
Figure 10: Photostimulation of the retrotrapezoid nucleus in control animals has no effect on breathing.

A. Location of the bilateral fiber optic tips identified in control rats ( $A r c h ; N=5)$ and Archexpressing rats $\left(\mathrm{Arch}^{+} ; \mathrm{N}=8\right.$ ) overlap in the ventrolateral medulla. These sites are plotted on two transverse brain sections closest to their location. The tip sites of example B are represented by the filled star. Stereotaxic coordinates (transverse planes posterior to Bregma) correspond to the atlas of (Paxinos \& Watson, 2005). B. Bilateral illumination of the RTN region with green light (10 s, $5 \mathrm{~mW}$ ) in an Arch rat produced no change in breathing variables. C. Group data from five Arch ${ }^{-}$animals show no effect of photostimulation of RTN with constant green light (10 $\mathrm{s}, \sim 5 \mathrm{~mW}$ ) on breathing variables in either non-REM sleep or quiet wake states (two-way RM ANOVA).

Author Contributions: P.G.G., R.L.S., P.G.R.B., R.K. conception and design of research; P.G.R.B, R.K., T.M.B., W.M.H, K.E.V. performed experiments; P.G.R.B., R.K., T.M.B., R.L.S. analyzed data; T.M.B., P.G.R.B., R.K., P.G.G. prepared figures; P.G.G., P.G.R.B., R.K. drafted manuscript; P.G.G., R.K., P.G.R.B., R.L.S. edited and revised manuscript; P.G.G., P.G.R.B., R.K., T.M.B., R.L.S. interpreted results of experiments; P.G.G. approved final version of manuscript. 


\section{Chapter 4: Is plasticity within the retrotrapezoid nucleus responsible for the recovery of the PCO2 set-point after carotid body denervation in rats?}

\section{This chapter is a re-print of the Journal of Physiology (2016) article (Basting et al., 2016).}

\subsection{Introduction}

The carotid bodies (CBs) encode arterial $\mathrm{PO}_{2}$ in a $\mathrm{pH}$-dependent manner and their activation during hypoxia stimulates breathing and the cardiovascular system (Nurse, 2014; Prabhakar \& Semenza, 2015). The CBs may also contribute to resting ventilation under normoxic conditions (Dejours, 1962; Blain et al., 2010; Smith et al., 2010; Hodges \& Forster, 2012). The main supportive evidence for $C B$ contribution to resting ventilation under normoxia is that carotid body denervation ( $\mathrm{CBD}$ ) produces a long-lasting (days to weeks) hypoventilation and elevates the steady-state level of arterial $\mathrm{PCO}_{2}\left(\mathrm{PaCO}_{2}\right)$ in all mammals, humans included (Forster \& Smith, 2010; Hodges \& Forster, 2012; Mouradian et al., 2012). Also, in unanesthetized dogs, resting ventilation is reduced and arterial $\mathrm{PCO}_{2}$ rises when an isolated $\mathrm{CB}$ is perfused with hyperoxic blood (Blain et al., 2009, 2010).

The hypoventilation and hypercapnia consecutive to CBD decline with time and the hypoxic ventilatory reflex (HVR) recovers to a variable extent. The degree and time-course of the recovery are species-dependent (Martin-Body et al., 1986; Forster, 2003; Timmers et al., 2003; Hodges \& Forster, 2012; Mouradian et al., 2012; Angelova et al., 2015). The recovery of the HVR has two potential causes. Accessory peripheral chemoreceptors (ectopic glomus cells, aortic bodies, others) (Dejours, 1962; McDonald \& Blewett, 1981) may gradually become more 
sensitive to hypoxia, develop new CNS connections and eventually compensate for the absence of carotid body input. In rats, the brainstem respiratory network may develop the ability to respond directly to hypoxia after CBD (Angelova et al., 2015) but this does not appear to be the case in mice; in this species the HVR was permanently abolished by deleting a gene that encodes a protein required for $\mathrm{O}_{2}$ sensing by the $\mathrm{CBs}$ but absent from the brain (Olf58 knock out) (Chang et al., 2015).

The surgical procedure used to remove the carotid bodies and their innervation may influence the degree and duration of the subsequent hypoventilation and hypercapnia (Olson et al., 1988a; Mouradian et al., 2012). Therefore, collateral damage to structures other than the carotid bodies may also contribute to the breathing changes. In fact, the return to normal ventilation and $\mathrm{PCO}_{2}$ seems poorly correlated with the recovery of a ventilatory reflex to cyanide or hypoxia (Forster, 2003; Hodges et al., 2005; Mouradian et al., 2012). This discrepancy implies that some form of central plasticity of the respiratory network unrelated to oxygen sensing develops. This interpretation is supported by evidence of neurochemical changes in the brainstem and of changes in the respiratory stimulation elicited by activating raphe neurons (Hodges et al., 2005; Mouradian et al., 2012; Miller et al., 2013).

In the present study we test whether a compensatory increase in the activity of central respiratory chemoreceptors contributes to the recovery of ventilation and the return to control of the arterial $\mathrm{PCO}_{2}$ set-point after CBD. We focused on a well characterized group of chemoreceptors: the retrotrapezoid nucleus (RTN). RTN neurons encode $\mathrm{CNS}^{\mathrm{PCO}_{2}}$ and operate as a nodal point for the regulation of breathing by $\mathrm{CO}_{2}$ (Gourine et al., 2010; Basting et al., 2015; Guyenet \& Bayliss, 2015). Inhibiting these neurons briefly (10 s) produces a hypopnea whose 
magnitude is a measure of their contribution to ventilation at the precise instant when the inhibitory stimulus is applied (Basting et al., 2015). Here we use the same optogenetic approach to test the following two hypotheses. First, after $C B D$, the contribution of RTN neurons to ventilation remains proportional to arterial $\mathrm{pH}$ or $\mathrm{PCO}_{2}$. Second, a week after $\mathrm{CBD}$, breathing is back to normal because RTN neurons are more active and this increased activity compensates for the loss of carotid body input.

\subsection{Methods}

\section{Animals and ethical approval}

All procedures conformed to the NIH Guide for the Care and Use of Laboratory Animals as well as and were approved by the University of Virginia Animal Care and Use Committee. The investigators understand the ethical principles under which the Journal of Physiology operates and our work complies with the animal ethics checklist provided by this journal. Experiments were performed on male Sprague-Dawley rats ( $n=24 ; 400-550 \mathrm{gm}$, Taconic, USA). Animals were housed under standard $12 \mathrm{~h}$ light/dark cycle with ad libitum access to food and water.

\section{Lentiviral construct and vector preparation}


We used a lentiviral vector (LVV) that expresses the photoactivatable proton pump ArchT3.0 (Chow et al., 2010; Han et al., 2011; Mattis et al., 2012) under the Phox2b-responsive promoter PRSx8 (pLenti-PRSX8 ArchT3.0-eYFP). PRSx8-ArchT-eYFP was generated by replacing the CamKIla promoter present in an existing pLenti CamKIla ArchT 3.0 eYFP construct (courtesy of K. Deisseroth via Addgene) by the PRSx8 promoter (kindly provided by M. Raizada, University of Florida). The LVV was produced by the University of North Carolina virus core at $3.0 \times 10^{8}$ viral particles per ml.

\section{Injections of vector}

The LVV was microinjected bilaterally under the facial motor nucleus in 14 rats as described previously (Basting et al., 2015). Rats were anesthetized with a mixture of ketamine (75 mg/kg), xylazine (5 mg/kg) and acepromazine $(1 \mathrm{mg} / \mathrm{kg}$ ) given inter-peritoneally (i.p.). Depth of anesthesia was assessed by an absence of the corneal and hind-paw withdrawal reflex. Additional anesthetic was administered as necessary ( $25 \%$ of the original dose, i.p. or intramuscular during surgery). Body temperature was kept close to $37^{\circ} \mathrm{C}$ with a servo-controlled heating pad and a blanket. All surgical procedures were performed under aseptic conditions. The hair over the rat's skull, neck and cheek were removed and skin disinfected. A skin incision was made over the left mandible to expose the facial nerve. This nerve was later stimulated to elicit antidromic field potentials in the facial motor nucleus (Brown \& Guyenet, 1985) . The rat was then placed prone on a stereotaxic apparatus (bite bar set at $-3.0 \mathrm{~mm}$ for flat skull; David Kopf Instruments). A $1.5 \mathrm{~mm}$ diameter hole was drilled into the occipital plate on both sides caudal to the parieto-occipital suture. The LVV solution was loaded into a $1.2 \mathrm{~mm}$ internal diameter glass pipette broken to a 25 micron tip (external diameter). Antidromic field potentials were recorded through the pipette in order to identify the location of the RTN which resides 
immediately below the facial motor nucleus. All injections were made 50-100 $\mu \mathrm{m}$ below this nucleus. The vector was pressure-injected bilaterally into five sites separated by $200 \mu \mathrm{m}$ (total volume $500-700 \mathrm{nl} / \mathrm{side})$. The caudal-most site was located below the caudal end of the facial motor nucleus (mean stereotaxic coordinate: $2.6 \mathrm{~mm}$ caudal to the parieto-occipital suture, 2.0 $\mathrm{mm}$ lateral to the midline, $8.3 \mathrm{~mm}$ below the cerebellar surface; individual coordinates varied by up to $400 \mu \mathrm{m}$ in every direction according to the location of the facial motor nucleus).

Next, we implanted electrodes for recording the electroencephalogram (EEG), neck electromyogram (EMG) and we inserted two optical fiber/ ferrule assemblies for light stimulation of ArchT-expressing neurons (one on each side, for further details see (Basting et al., 2015)). The bottom of the optical fibers ( $200 \mu \mathrm{m}$ diameter) were placed $300 \mu \mathrm{m}$ dorsal to the middle of the LVV injection sites. The head-stage (EEG and EMG connections) and optic fiberferrules were secured to the skull using a 2-part epoxy (Loctite). Incisions were then closed in two layers (muscle and skin) with absorbable sutures and vet bond adhesive. Rats received postoperative ampicillin (125 mg/kg, i.p.) and ketoprofen (3-5 mg/kg, s.c.) and were monitored daily. Rats recovered for four weeks for functional expression of the opsin.

\section{Carotid body excision and denervation (CBD)}

Eleven rats were subjected to CBD and seven animals had sham surgery. The first group included seven of the rats that had received LVV injections 4-6 weeks prior. CBD and sham surgery were done under aseptic conditions with body temperature maintained close to $37^{\circ} \mathrm{C}$. Anesthesia was induced with $5 \%$ isoflurane anesthesia in pure oxygen and maintained with $2 \%$ isoflurane in oxygen during surgery. Depth of anesthesia was assessed by an absence of the corneal and hind-paw withdrawal reflex. The CBs were isolated following a mid-line incision in 
the ventral surface of the neck and physically removed with forceps. In addition, the carotid sinus nerves were cut and phenol was applied to the carotid bifurcation. This surgical procedure also destroyed the carotid baroreceptors. The incisions were then closed in two layers (muscle and skin) with absorbable sutures and vet bond adhesive. Rats received post-operative ampicillin (125 mg/kg, i.p.) and ketoprofen (3-5 mg/kg, s.c.) and monitored daily. Sham surgery consisted of exposing the carotid bifurcation bilaterally without damaging the carotid bodies nor surrounding nerves. All rats were given 6-7 days to recover from surgery before their respiratory chemoreflex and ventilatory response to bilateral RTN inhibition was retested.

\section{Physiological experiments in freely-behaving rats with unrestrained whole body plethysmography}

The animals were tested in a Buxco plethysmography chamber modified to allow tethered EEG/EMG recordings and optical stimulation. Before the actual experiments were run the rats were repeatedly habituated to these surroundings which were visually-isolated and with low-ambient noise. Low breathing rates under normoxia (65-80 bpm) and a regular sleep pattern were taken as evidence of low stress on experiment days. On those days, rats were lightly anesthetized with isoflurane (induction with $5 \%$, maintenance with $2 \%$ in pure oxygen for $<1 \mathrm{~min}$ ) to permit cleaning of hardware and connection to the ferrule and EEG/EMG recording assembly. A $200 \mu$ m-thick multimode optical fiber terminated with a ferrule was mated to each of the two implanted ferrules with a zirconia sleeve. Optical matching gel (Fiber Instrument Sales) was applied at the ferrule junction to reduce light loss. A minimum of $1 \mathrm{hr}$ was allowed for recovery from anesthesia and the emergence of stable sleep/wake patterns. Recordings were made between 10 am- 6 pm with a minimum of three days rest between tests. The ventilatory 
response to RTN inhibition was assessed using barometric, unrestrained whole-body plethysmography (EMKA technologies). The plethysmography chamber was continuously flushed with $1.5 \mathrm{~L} / \mathrm{min}$ of $\mathrm{O}_{2}$ balanced with $\mathrm{N}_{2}$ and $\mathrm{CO}_{2}(3 \%)$ was added occasionally. The gas mixture was regulated by computer-driven mass flow controllers for $\mathrm{O}_{2}, \mathrm{~N}_{2}$ and $\mathrm{CO}_{2}$ (Alicat). Temperature and humidity within the plethysmography chamber were kept constant $\left(25^{\circ} \mathrm{C}\right.$ and $42 \%$ relative humidity).

Photoinhibition of ArchT3.0-expressing neurons was achieved with a green laser (532 nm, Shanghai Laser and Optics Century) controlled by a Grass model S88 stimulator (AstroMed Inc.). The green light was applied in $10 \mathrm{~s}$ episodes of continuous illumination. The transmission efficiency of each implantable optical fiber was tested prior to implantation with a light meter (Thorlabs). Based on this test, the power of the laser was adjusted so that the final output at the tip of the implanted fiber was estimated between 6-7 $\mathrm{mW}$ in vivo.

\section{Indwelling femoral artery catheter implantation and blood gas measurements.}

Blood gases were measured in 16 rats. This cohort included six fully instrumented rats subjected to carotid body denervation seven days prior, another six fully instrumented rats that had been sham-operated (also 7 days prior) and 4 uninstrumented rats (without vector injection, optical fiber or head-set for EEG measurements). An indwelling femoral artery catheter was implanted while the rats were anesthetized with isoflurane ( $5 \%$ induction and $2 \%$ for maintenance of anesthesia in pure oxygen). The hair on the rat's upper back, neck and right inner leg was shaved and the skin disinfected. One incision on the rat's inner leg was made to expose the femoral artery and another one was made between the shoulder blades to exteriorize the catheter. A catheter (PE50) filled with 100U/ml heparinized saline was implanted 
into the right femoral artery. The catheter was threaded subcutaneously from the femoral artery along the rat's back to emerge between the rats shoulder blades. Incisions were then closed in two layers (muscle and skin) with absorbable sutures and vet bond adhesive, bupivacaine was infiltrated around the skin incisions and the rats received the standard postoperative doses of ketoprofen and ampicillin (see above). The animal was then placed in the plethysmography chamber and the catheter was connected through a harness to a swivel system that allowed the animal to move freely within the chamber and the experimentor to withdraw blood without having to disturb the animal. After three hours of recovery within the chamber, the animal was exposed to the various hypoxic or hypercapnic gas mixtures of interest for 30 minutes to insure that a steady-state had been achieved. Arterial blood $(0.2 \mathrm{~mL})$ was then withdrawn and arterial blood gases and other parameters $\left(\mathrm{pH}, \mathrm{PCO}_{2}, \mathrm{PO}_{2}\right.$ and $\left.\mathrm{HCO}_{3}{ }^{-}\right)$were measured with a VetScan Blood Gas Analyzer. To maintain patency of the line the catheter was flushed with $100 \mathrm{U} / \mathrm{ml}$ heparinized saline after each blood withdrawal. After all blood sampling had been performed (5-6 hrs after catheter implantation) the animals were killed with an overdose of anesthetic ( $5 \%$ isoflurane delivered to the chamber followed by an intra-arterial injection of 70-90 mg/kg of Nembutal. The rats were then perfused with aldehyde and their brains harvested for histology (details later).

\section{Data acquisition and analysis}

Physiological signals were processed using Spike v7.03 software (Cambridge Electronic Design). EEG and EMG were amplified and band pass filtered (EEG: 0.1-100 Hz, x1000. EMG: $100-3000 \mathrm{~Hz}, x 1000)$ and acquired at a sampling frequency of $1 \mathrm{KHz}$. The signal generated by the differential pressure transducer connected to the plethysmography chamber was amplified and band pass filtered $(0.1-15 \mathrm{~Hz}, \mathrm{x} 100)$ and acquired at a sampling frequency of $1 \mathrm{KHz}$. Satisfactory 
EEG/EMG recordings were obtained in $8 / 14$ rats. In these rats, periods of wake or natural sleep were classified on the basis of EEG, EMG activity, the patterns of breathing activity and the animals' posture. During non-rapid eye movement (nREM) sleep, EEG spectra were dominated by delta activity $(0.5-4 \mathrm{~Hz})$ with little or no EMG activity, and a stable breathing pattern. Quiet wake was characterized by a reduction in total power with EMG tone and labile breathing. REM sleep was characterized by a theta activity $(6-7 \mathrm{~Hz})$, loss of EMG and labile breathing. In the other 6 rats in which EEG/EMG recordings were not available, RTN inhibition was performed when the breathing rate was regular and the animals either immobile or exhibiting the curled up posture characteristic of sleep. Light was not applied during REM sleep, a state that was instantly recognizable with or without EEG recording by the combination of a sleeping posture and breathing irregularities. A minimum of 6 photoactivation trials were conducted in nREM and/or quiet wake at each level of inspired $\mathrm{O}_{2}\left(\mathrm{FiO}_{2} 65 \%, 21 \%, 18 \%, 15 \%\right.$ or $12 \%$, balance $\mathrm{N}_{2}$, or $\mathrm{FiO}_{2}$ $21 \%+\mathrm{FiCO}_{2} 3 \%$ balance $\mathrm{N}_{2}$ ) and the responses were averaged. Trials were conducted during nonREM sleep or quiet waking only. No trial was conducted during REM sleep, a state in which breathing is labile and the contribution of RTN to breathing is drastically reduced (Burke et al., 2015b). Trials during which the animals moved, as indicated by EMG activity, were also ignored. For each trial, breathing parameters were measured during the $10 \mathrm{~s}$ preceding light delivery (baseline parameters) and while the light was applied (10s). Breathing frequency $\left(F_{R}\right.$, breaths/min, bpm) and tidal volume $\left(\mathrm{V}_{\mathrm{T}}, \mathrm{ml}\right.$ per $100 \mathrm{~g}$ body weight, $\left.\mathrm{BW}\right)$ were calculated using Spike software v7.3 (CED). $V_{T}$ was derived from the integral of the flow signal during inspiration following calibration to waveforms generated by injecting $5 \mathrm{ml}$ of dry air into the chamber. Minute ventilation $\left(M V=F_{R}{ }^{*} V_{T}\right.$ ) was expressed as $\left.\mathrm{ml} / 100 \mathrm{~g} \mathrm{BW} / \mathrm{min}\right)$. Event triggered breathing responses (Figure 6) were an average of 3-6 photoactivation trials at each level of inspired $\mathrm{O}_{2}$ for 
each of the 7 rats both before and after CBD. Sigh data were collected in CBD-denervated or sham-denervated rats for a total of 10 min under each above described gas condition when the animal was quietly awake or in nonREM sleep.

\section{Histology}

The animals that had received injections of vector were deeply anesthetized with $5 \%$ isoflurane followed by a lethal dose of ketamine/xylazine/acepromazine (75/5/1 mg/kg i.m.) or, in the case of the animals instrumented with intra-arterial catheters with $5 \%$ isoflurane followed by a lethal dose of Nembutal (70-80 mg intra-arterially). Then, these animals were perfused transcardially with $4 \%$ paraformaldehyde, brains removed and processed as described previously (Abbott et al., 2012). Immunohistochemistry with antibodies against tyrosine hydroxylase (sheep anti-TH, 1: 2000, Millipore), eYFP (to detect ArchT3.0; chicken anti-GFP, 1:1000, AVES labs) and Phox2b, a marker of RTN chemoreceptors (rabbit anti- Phox2b, 1:8000, a gift from J.F. Brunet, Ecole Normale Superieure, Paris, France) were performed as previously described (Abbott et al., 2009). Cell mapping and counting and photography were done using the Neurolucida system (MicroBrightfield, Inc, Colchester, VT) with a Zeiss Axioskop microscope driven stage and Zeiss MRc camera. Cell counts were taken from a 1 in 6 series of sections and only profiles containing a nucleus were counted.

\section{Statistics}

Differences within and between groups were determined using two-tailed paired Student's t-test, one-way repeated measures ANOVA with Dunnett's multiple comparisons or two-way repeated measures ANOVA with Sidak's multiple comparisons in Graphpad Prism v. 6. All group data, between and within groups, passed the Shapiro-Wilkes test on error residuals for 
normality as determined using the R statistical software package. Results are generally expressed as mean with standard deviation (SD). Mean without SD is represented in plots in which individual values are shown. Statistical significance is denoted by one star $(P<0.05)$, two stars $(\mathrm{P}<0.01)$ or three stars $(\mathrm{P}<0.005)$.

\subsection{Results}

\section{Arterial blood gases in control and carotid body denervated (CBD) rats exposed to various $\mathrm{O}_{2}$} concentrations

Blood gases $\left(\mathrm{PCO}_{2}, \mathrm{PO}_{2}\right), \mathrm{pH}$ and $\left[\mathrm{HCO}_{3}^{-}\right]$were measured while rats were exposed to $65 \%, 21 \%, 15 \%, 12 \% \mathrm{FiO}_{2}$ and $12 \% \mathrm{FiO}_{2}$ plus $3 \% \mathrm{FiCO}_{2}$ within the plethysmography chamber. This particular $\mathrm{FiCO}_{2}$ level was selected because it very nearly compensates the respiratory alkalosis elicited by $12 \% \mathrm{FiO}_{2}$. The measurements were made in six of the $7 \mathrm{CBD}$ rats 7 days after neck surgery and in six rats with intact carotid bodies. The rats were habituated to their surroundings and blood was remotely withdrawn while the animals were quiescent, as monitored by visual inspection and the plethysmography signal. Under hyperoxia and normoxia rats were either asleep or quietly awake. Under hypoxia $\left(12 \% \mathrm{FiO}_{2}\right)$ rats were typically quietly awake.

In the 6 control rats, graded hypoxia produced graded respiratory alkalosis (blood alkalization, hypocapnia and reduced $\left[\mathrm{HCO}_{3}^{-}\right]$) whereas hyperoxia caused a minor respiratory acidosis (Figure 1A, B, D, Table 1). These effects were evaluated by one-way repeated-measures ANOVA across four levels of $\mathrm{FiO}_{2}$ and were highly significant (for $\mathrm{pH}: p=0.001$; Dunnett's multiple comparisons for $\mathrm{pH}: 21 \%$ vs. $65 \% \mathrm{FiO}_{2}, p=0.0104 ; 21 \%$ vs. $15 \% \mathrm{FiO}_{2}, p<0.0001 ; 21 \%$ vs 
$12 \% \mathrm{FiO}_{2}, p<0.0001$; for $\mathrm{PCO}_{2}: p<0.0001$; Dunnett's multiple comparisons for $\mathrm{PCO}_{2}: 21 \%$ vs. $65 \% \mathrm{FiO}_{2}, p=0.0551 ; 21 \%$ vs. $15 \% \mathrm{FiO}_{2}, p=0.0184 ; 21 \%$ vs $12 \% \mathrm{FiO}_{2}, p=0.0004$; for $\mathrm{HCO}_{3}: p<$ 0.0001; Dunnett's multiple comparisons for $\mathrm{HCO}_{3}: 21 \%$ vs $65 \% \mathrm{FiO}_{2}, p=0.4159 ; 21 \%$ vs. $15 \%$ $\mathrm{FiO}_{2}, p=0.0120 ; 21 \%$ vs $\left.12 \% \mathrm{FiO}_{2}, p<0.0001\right)$. In $6 \mathrm{CBD}$ rats, one-way repeated measures ANOVA also showed a significant overall effect of $\mathrm{FiO}_{2}$ on arterial blood $\mathrm{pH}, \mathrm{PCO}_{2}$, and $\left[\mathrm{HCO}_{3}{ }^{-}\right]$ but Dunnett's multiple comparison test revealed that significant group differences from room air conditions were only present at $12 \% \mathrm{FiO}_{2}\left(\mathrm{pH}: \mathrm{F}=18.29, p=0.0004,21 \%\right.$ vs. $12 \% \mathrm{FiO}_{2}, p=$ $0.008 ; \mathrm{PCO}_{2}: \mathrm{F}=32.51, p<0.0001,21 \%$ vs $12 \% \mathrm{FiO}_{2}, p=0.0009 ; \mathrm{HCO}_{3}{ }^{-}: \mathrm{F}=12.99, p=0.0068$ $21 \%$ vs $12 \% \mathrm{FiO}_{2}, p=0.0326$ ) (Figure $\left.1 \mathrm{~A}, \mathrm{~B}, \mathrm{D}\right)$.

The effect of $\mathrm{CBD}$ on arterial blood parameters $\left(\mathrm{pH}, \mathrm{PCO}_{2}, \mathrm{PO}_{2}\right.$, and $\left.\left[\mathrm{HCO}_{3}{ }^{-}\right]\right)$were evaluated with two-way repeated-measures ANOVA for two factors $\left(\mathrm{FiO}_{2}, 4\right.$ levels; rat group i.e. CBD vs.intact; $\mathrm{N}=6$ per group) (Figure 1 ). There were significant differences of arterial $\mathrm{pH}$ between control and $\mathrm{CBD}$ animals except under normoxia (effect of $\mathrm{FiO}_{2}$ on $\mathrm{pH}, p<0.0001$; effect of CBD on $\mathrm{pH}, p<0.0322$; interaction, $p<0.0001$; Sidak's multiple comparisons for $\mathrm{pH}$ before and after CBD: $65 \% \mathrm{FiO}_{2}, p=0.0048 ; 21 \% \mathrm{FiO}_{2}, p>0.9 ; 15 \% \mathrm{FiO}_{2}, p=0.008 ; 12 \% \mathrm{FiO}_{2}, p<$ 0.0001). Significant differences in arterial $\mathrm{PCO}_{2}$ were also identified (effect of $\mathrm{FiO}_{2}$ on $\mathrm{PCO}_{2}, p<$ 0.0001; effect of $\mathrm{CBD}$ on $\mathrm{PCO}_{2}, p=0.0987$; interaction, $p<0.0001$; Sidak's multiple comparisons for arterial $\mathrm{PCO}_{2}$ in intact vs. $\mathrm{CBD}$ rats: $65 \% \mathrm{FiO}_{2}, p=0.119 ; 21 \% \mathrm{FiO}_{2}, p>0.9 ; 15 \% \mathrm{FiO}_{2}, p=$ $0.0128 ; 12 \% \mathrm{FiO}_{2}, p=0.0002$ ) (Figure 1B). Significant differences in arterial $\mathrm{HCO}_{3}{ }^{-}$were also found after $\mathrm{CBD}$ (effect of $\mathrm{FiO}_{2}$ on $\mathrm{HCO}_{3}{ }^{-}, p<0.0001$; effect of $\mathrm{CBD}$ on $\mathrm{HCO}_{3}{ }^{-}, p=0.3204$; interaction, $p<0.0019)$. The statistical analysis revealed no effect of $\mathrm{CBD}$ on arterial $\mathrm{PO}_{2}($ effect of $\mathrm{CBD}$ on $\mathrm{PO}_{2}, p=0.3803$; interaction, $p=0.6704$ ) (Figure $1 \mathrm{C}$ ). Yet, $\mathrm{CBD}$ animals had consistently lower $\mathrm{PO}_{2}$ levels under $12 \%$ hypoxia (effect of $\mathrm{FiO}_{2}$ on $\mathrm{PO}_{2}, \mathrm{p}<0.0001 ;$ Table 1 ). 
In summary, exposure to hypoxia in intact quietly resting rats produced the expected respiratory alkalosis. After $\mathrm{CBD}$, the alkalosis was much reduced but not eliminated. At $12 \% \mathrm{FiO}_{2}$, hypoxia still produced a slight but significant rise in arterial $\mathrm{pH}$ and a decrease of both arterial $\mathrm{PCO}_{2}$ and $\left[\mathrm{HCO}_{3}{ }^{-}\right]$relative to room air conditions. Arterial $\mathrm{PO}_{2}$ was slightly lower at $15 \%$ and $12 \%$ $\mathrm{FiO}_{2}$ in $\mathrm{CBD}$ vs. intact rats (Figure $1 \mathrm{C}$ ) but the difference did not reach statistical significance.

\section{Effect of $\mathrm{FiO}_{2}$ on breathing in $\mathrm{CBD}$ or sham-operated rats}

The effects of $C B D$ on the ventilatory response to hypoxia or hyperoxia (Figure $2 A$ ) were evaluated with two-way repeated measures ANOVA for two factors $\left(\mathrm{FiO}_{2}, 4\right.$ levels; rat group, pre- vs. post sham or CBD surgery; 7 rats per group). There was a significant effect of $\mathrm{FiO}_{2}$ and $\mathrm{CBD}$ on $\mathrm{F}_{\mathrm{R}}\left(\mathrm{FiO}_{2}\right.$ effect, $p<0.0001 ; \mathrm{CBD}$ effect, $p=0.0009$; interaction, $p=0.0105$; Sidak's multiple comparisons for $\mathrm{F}_{\mathrm{R}}$ before vs. after $\mathrm{CBD}: 65 \% \mathrm{FiO}_{2}, p=0.006 ; 21 \% \mathrm{FiO}_{2}, p=0.004 ; 15 \%$ $\left.\mathrm{FiO}_{2}, p<0.0001 ; 12 \% \mathrm{FiO}_{2}, p<0.0001\right)$. There was a significant overall effect of $\mathrm{FiO}_{2}$ on $\mathrm{V}_{\mathrm{T}}(p<$ 0.0001 ; the trend was towards smaller $V_{\mathrm{T}}$ under hypoxia) but no overall effect of $\operatorname{CBD}$ on $\mathrm{V}_{\mathrm{T}}(p=$ $0.6256)$ and no interaction $(p=0.3445)$. Finally, there was a significant effect of $\mathrm{FiO}_{2}$ and $\mathrm{CBD}$ on $\mathrm{V}_{\mathrm{E}}$ (effect of $\mathrm{FiO}_{2}, p=0.0002$; effect of $\mathrm{CBD}, p=0.0322$; interaction, $p=0.0002$; Sidak's multiple comparisons for $\mathrm{V}_{\mathrm{E}}$ before vs. after $\mathrm{CBD}: 65 \% \mathrm{FiO}_{2}, p=0.3062 ; 21 \% \mathrm{FiO}_{2}, p>0.9 ; 15 \% \mathrm{FiO}_{2}, p=$ $0.0028 ; 12 \% \mathrm{FiO}_{2}, p<0.0001$ ) (Figure $2 \mathrm{~A}$ ). Sham denervation had no effect on the ventilatory response to hypoxia (Figure 2B) (two-way RM ANOVA: $\mathrm{FiO}_{2}$ effect, $p<0.0001$; $\mathrm{SCBD}$ effect, $p=$ 0.3932; interaction, $p=0.8742$ ). Before denervation, $\mathrm{F}_{\mathrm{R}}$ was significantly higher at $12 \% \mathrm{FiO}_{2}$ than at $21 \% \mathrm{FiO}_{2}(p=0.0002)$ and $\mathrm{V}_{\mathrm{E}}$ was significantly higher at $12 \% \mathrm{FiO}_{2}$ compared to normoxia as well $(p=0.0013)$. However, $\mathrm{FiO}_{2}$ had no effect on $\mathrm{V}_{\mathrm{T}}$ in these intact rats. After denervation 
hypoxia had very little effect on any of the breathing parameters with only a slight increase in $F_{R}$ in $12 \% \mathrm{FiO}_{2}$ (One-way RM ANOVA: After denervation $21 \% \mathrm{FiO}_{2}$ vs $12 \%, p=0.0013$ ). Of note, CBD significantly reduced $F_{R}$ under normoxia but had no effect on $V_{T}$ or $V_{E}$.

In brief, the hypoxic ventilatory response consisted primarily of an increase in $F_{R}$. CBD but not sham surgery attenuated the hypoxic ventilatory response. The $F_{R}$ increase in hypoxia, although greatly reduced, remained statistically significant after $\mathrm{CBD}$ in $12 \% \mathrm{FiO}_{2}$. When combined with the slight respiratory alkalosis revealed by blood gas analysis, this result suggests that a small ventilatory stimulation persisted at $12 \% \mathrm{FiO}_{2}$ in $\mathrm{CBD}$ rats.

\section{Effects of CBD on hypoxia-induced sighs}

Sigh frequency was measured before and after CBD in six different gas conditions (65\% $\left.\mathrm{FiO}_{2}, 21 \% \mathrm{FiO}_{2}, 21 \% \mathrm{FiO}_{2}+3 \% \mathrm{FiCO}_{2}, 15 \% \mathrm{FiO}_{2}, 12 \% \mathrm{FiO}_{2}, 12 \% \mathrm{FiO}_{2}+3 \% \mathrm{FiCO}_{2}\right)$. Before CBD, sigh incidence increased significantly above control $\left(\mathrm{FiO}_{2} 21 \%\right)$ at $15 \%$ and $12 \% \mathrm{FiO}_{2}$. The addition of $3 \% \mathrm{FiCO}_{2}$ had no effect on the sigh frequency under normoxia but eliminated the increase sigh incidence caused by $12 \% \mathrm{FiO}_{2}$ (Figure 3). CBD attenuated the increase in sigh frequency produced by hypoxia (Figure 3). Statistical analysis (2-way RM ANOVA with factors $\mathrm{FiO}_{2}$ and CBD) showed a highly significant effect of $\mathrm{FiO}_{2}$ on sigh incidence, $p<0.0001$, a highly significant effect of CBD on sighs, $p=0.0086$, and a highly significant interaction, $p<0.0001$. Sidak's multiple comparisons before vs. after CBD showed no effect of $C B D$ on sigh incidence at $65 \%$ $\mathrm{FiO}_{2}(p=0.8115), 21 \% \mathrm{FiO}_{2}(p=0.2018)$ or in $21 \% \mathrm{FiO}_{2}+3 \% \mathrm{CO}_{2},(p>0.9)$. Sigh incidence was markedly reduced by $\mathrm{CBD}$ at $15 \% \mathrm{FiO}_{2}(p=0.0122)$, and $12 \% \mathrm{FiO}_{2},(p<0.0001)$ but unaffected when the rats were exposed to $12 \% \mathrm{FiO}_{2}+3 \% \mathrm{FiCO}_{2}(p=0.9085)$ (Figure 3). In carotid body 
intact rats sigh frequency was significantly elevated above the normoxic baseline only under $12 \% \mathrm{FiO}_{2}(\mathrm{P}=0.014)$. The addition of $3 \% \mathrm{FiCO}_{2}$ returned sigh frequency to baseline. The difference between $15 \% \mathrm{FiO}_{2}$ and $12 \% \mathrm{FiO}_{2}+3 \% \mathrm{FiCO}_{2}$ was small but statistically significant $(\mathrm{P}=0.03)$.

\section{Effect of CBD on the breathing response to ArchT3.0 photoactivation}

In order to assess the instant contribution of ArchT-transduced neurons to breathing in conscious rats we measured the degree to which $F_{R}, V_{T}$ and $V_{E}$ were reduced during 10 seconds of light-induced bilateral inhibition of these neurons. These measurements were made before and 6-7 days after CBD in 7 rats. As illustrated in a representative example (Figure 4), ArchT activation inhibited both $F_{R}$ and $V_{T}$ in intact rats. $F_{R}$ as well as $V_{T}$ inhibition was greater in hyperoxia than in normoxia and smaller in hypoxia than in normoxia. These results are identical to those obtained in a prior study (Basting et al., 2015). Following CBD, breathing inhibition was about the same as before CBD under hyperoxia but considerably larger under normoxia or hypoxia. In order to control for the possibility that the observed differences might have resulted from the passage of time, for example because of a higher level of expression of ArchT, we performed the identical experiment in 7 rats that were operated but in which the CBs and their innervation were left intact. Breathing responses before and after sham surgery were indistinguishable (representative case not illustrated).

The group data are illustrated in Figure 5. In CBD rats, ArchT activation produced significantly more breathing inhibition after surgery (Figure $5 \mathrm{~A}$; effect of $\mathrm{FiO}_{2}$ on delta $\mathrm{F}_{\mathrm{R}}, p<$ 0.0001; effect of CBD on delta $\mathrm{F}_{\mathrm{R}}, p=0.0169$; interaction, $p=0.6274$; Sidak's multiple comparisons for delta $\mathrm{F}_{\mathrm{R}}$ in sham vs. $\mathrm{CBD}$ rats: $65 \% \mathrm{FiO}_{2}, p=0.2698 ; 21 \% \mathrm{FiO}_{2}, p=0.0119 ; 15 \%$ 
$\mathrm{FiO}_{2}, p=0.0228 ; 12 \% \mathrm{FiO}_{2}, p=0.0085$. Effect of $\mathrm{FiO}_{2}$ on delta $\mathrm{V}_{\mathrm{T}}, p=0.0002$; effect of CBD on delta $\mathrm{V}_{\mathrm{T}}, p=0.0421$; interaction, $p=0.0604$; Sidak's multiple comparisons for delta $\mathrm{V}_{\mathrm{T}}$ in sham vs. $\mathrm{CBD}$ rats: $65 \% \mathrm{FiO}_{2}, p=0.0067 ; 21 \% \mathrm{FiO}_{2}, p=0.0008 ; 15 \% \mathrm{FiO}_{2}, p<0.0001 ; 12 \% \mathrm{FiO}_{2}, p<$ 0.0447. Effect of $\mathrm{FiO}_{2}$ on delta $\mathrm{V}_{\mathrm{E}}, p<0.0001$; effect of CBD on delta $\mathrm{V}_{\mathrm{E}}, p=0.0056$; interaction, $p$ $=0.0754$; Sidak's multiple comparisons for delta $\mathrm{V}_{\mathrm{E}}$ in sham vs. $\mathrm{CBD}$ rats: $65 \% \mathrm{FiO}_{2}, p=0.9855$; $21 \% \mathrm{FiO}_{2}, p=0.0113 ; 15 \% \mathrm{FiO}_{2}, p=0.0049 ; 12 \% \mathrm{FiO}_{2}, p=0.0042$ ). Of note, light-induced inhibition of $F_{R}$ and $V_{E}$ was increased after CBD in normoxia and in hypoxia but but not under hyperoxia (Figure 5A).

By contrast, sham-operated rats responded identically to ArchT activation one week after the operation (Figure $5 \mathrm{~B}$; effect of $\mathrm{FiO}_{2}$ on delta $\mathrm{F}_{\mathrm{R}}, p<0.0001$; effect of $\mathrm{CBD}$ on delta $\mathrm{F}_{\mathrm{R}}, p=$ 0.3117; interaction, $p=0.8023$. Effect of $\mathrm{FiO}_{2}$ on delta $\mathrm{V}_{\mathrm{T}}, p<0.0001$; effect of CBD on delta $\mathrm{V}_{\mathrm{T}}, p$ $=0.6660$; interaction, $p=0.2065$. Effect of $\mathrm{FiO}_{2}$ on delta $\mathrm{V}_{\mathrm{E}}, p<0.0001$; effect of CBD on delta $\mathrm{V}_{\mathrm{E}}$, $p=0.6387$; interaction, $p=0.1864)$.

In summary, photoinhibition of ArchT-expressing neurons reduced breathing to the same extent before and 6-7 days after sham neck surgery. As reported previously, the inhibition was greatest under hyperoxia and progressively smaller with increasing degrees of hypoxia (Basting et al., 2015). CBD resulted in an increase of the light-induced breathing inhibition at all $\mathrm{FiO}_{2}$ levels except in hyperoxia. Breathing frequency was greatly reduced by photostimulation after $C B D$ indicating that the photoinhibited RTN neurons regulate $F_{R}$ even in the absence of $C B$ input.

\section{Kinetics of the breathing response to ArchT activation in intact and CBD rats}


As illustrated in Figure 4, carotid body denervation changed the time course of the breathing responses to photoinhibition of ArchT-transduced neurons and this change was much more obvious under normoxia and hypoxia than under hyperoxia. In order to examine the kinetics of the breathing response with greater precision, event triggered responses $\left(F_{R}\right.$ and $\left.V_{T}\right)$ were averaged over $30 \mathrm{~s}$, a time frame that included the $10 \mathrm{~s}$ preceding and $10 \mathrm{~s}$ following the photostimulation period. The responses were first averaged within animals (3-5 repeats) and the resulting averages were grand-averaged across 7 rats (Figure 6). Under hyperoxia, the decrease of $F_{R}$ and $V_{T}$ produced by the photoinhibition was sustained throughout the entire illumination phase (10 s) and CBD made no difference to the kinetics (Figure 6). In pre and post CBD animals under hyperoxia, at the end of the inhibitory period, both $F_{R}$ and $V_{T}$ returned slowly towards the baseline with kinetics that could be modelled by an exponential function (preCBD $t_{1 / 2}=22.4 \pm$ 7.8; post $\mathrm{CBD} \mathrm{t}_{1 / 2}=22.8 \pm 2.2$ seconds $(\mathrm{N}=4$ rats, 3 trials per rat $)$ ).

In normoxia, the kinetics of the response to ArchT stimulation were notably different. Before CBD, after an initially large hypopnea, respiration started to recover towards the baseline within three seconds of the laser onset and returned close to the pre-illumination baseline by the end of the light stimulus. Breathing frequency rose slightly above control level when the light was switched off and returned to control level a few seconds later. After CBD, breathing inhibition during normoxia resembled the pattern observed under hyperoxia. Inhibition was sustained throughout the $10 \mathrm{~s}$ light delivery period and recovered approximately exponentially at the same rate as before CBD after the light was switched off $\left(t_{1 / 2}: 19.6 \pm 9\right.$ seconds; $N=4$ rats, 3 trials per rat). At $12 \% \mathrm{FiO}_{2}$, breathing inhibition by ArchT activation was negligible before $\mathrm{CBD}$. After $\mathrm{CBD}$, breathing inhibition was sustained during the entire laser on episode and recovered instantly after the laser was switched off. 


\section{Relationship between ArchT-induced breathing inhibition and blood gases in intact and CBD} rats

We wished to determine whether CBD changes the relationship between arterial blood gases and the contribution of ArchT-transduced neurons to $F_{R}, V_{T}$ and $V_{E}$. To do so we analyzed the relationship between the light-induced hypopnea $\left(\Delta \mathrm{F}_{\mathrm{R}}, \Delta \mathrm{V}_{\mathrm{T}}\right.$ and $\Delta \mathrm{V}_{\mathrm{E}}$ relative to baseline values recorded immediately prior to illumination ) and the level of arterial $\mathrm{pH}, \mathrm{PO}_{2}, \mathrm{PCO}_{2}$ and $\left[\mathrm{HCO}_{3}\right]$ (Figure 7). Before surgery, rats were exposed to $65 \%, 21 \%, 15 \%, 12 \% \mathrm{FiO}_{2}$ and $12 \% \mathrm{FiO}_{2}$ $+3 \% \mathrm{FiCO}_{2}$; after $\mathrm{CBD}$ they were exposed to the same gas mixtures as well as $18 \% \mathrm{FiO}_{2}$.

$\Delta \mathrm{F}_{\mathrm{R}}$ during ArchT activation was negatively correlated with arterial $\mathrm{pH}$ and positively correlated with arterial $\mathrm{PCO}_{2}$ and $\left[\mathrm{HCO}_{3}^{-}\right]$both before and after $\mathrm{CBD}$. These relationships appeared approximately linear. The slopes were consistently steeper after CBD but the slope change was not statistically significant (difference in slopes of $\Delta F_{R}$ vs. pH before and after CBD: $F$ $=1.217, p=0.2736$; difference in slopes of $\Delta \mathrm{F}_{\mathrm{R}} \mathrm{Vs} . \mathrm{PCO}_{2}$ before and after CBD: $\mathrm{F}=2.9746, p=$ 0.08881; difference in slopes of $\Delta \mathrm{F}_{\mathrm{R}} \mathrm{vs}$. $\left[\mathrm{HCO}_{3}{ }^{-}\right]$before and after $\mathrm{CBD}: \mathrm{F}=0.8541, p=0.3584$ ). $\Delta \mathrm{V}_{\mathrm{T}}$ and $\Delta \mathrm{V}_{\mathrm{E}}$ during ArchT activation were linearly related to arterial $\mathrm{pH}, \mathrm{PCO}_{2}$ and $\left[\mathrm{HCO}_{3}{ }^{-}\right]$in the same way as $\Delta \mathrm{F}_{\mathrm{R}}$. The slope of these relationships were also not detectably different after CBD (difference in slopes of $\Delta \mathrm{V}_{\mathrm{E}}$ vs. $\mathrm{pH}$ before and after CBD: $\mathrm{F}=0.6697, p=0.2645$; difference in slopes of $\Delta V_{E}$ vs. PCO2 before and after CBD: $F=1.559, p=0.2158$; difference in slopes of $\Delta V_{E}$ vs. $\left[\mathrm{HCO}_{3}{ }^{-}\right]$before vs. after $\mathrm{CBD}: \mathrm{F}=0.1343, p=0.7151$. difference in slopes of $\Delta \mathrm{V}_{\mathrm{T}}$ vs. pH before and after CBD: $\mathrm{F}=0.0 .2709, p=0.6043$; difference in slopes of $\Delta \mathrm{V}_{\mathrm{T}} \mathrm{vs} . \mathrm{PCO}_{2}$ before and after CBD: $\mathrm{F}=1.899, p=0.1724$; difference in slopes of $\Delta \mathrm{V}_{\mathrm{T}}$ vs. $\left[\mathrm{HCO}_{3}{ }^{-}\right]$before vs. after $\mathrm{CBD}: \mathrm{F}=$ $0.8586, p=0.3572$ ). The relationship between $\Delta \mathrm{V}_{\mathrm{E}}$ and arterial $\mathrm{PO}_{2}$ was left shifted after $C B D$ 
(Figure 7B). A non-linear regression analysis of these curves revealed a statistically significant effect of $C B D$ (difference in curves of $\Delta \mathrm{F}_{\mathrm{R}}$ before vs. after $\mathrm{CBD}$ in relation to $\mathrm{PO}_{2}: \mathrm{F}=5.09, p=$ 0.003; difference in curves of $\Delta \mathrm{V}_{\mathrm{T}}$ before vs. after $\mathrm{CBD}$ in relation to $\mathrm{PO}_{2}: \mathrm{F}=7.804, p=0.0001$; difference in curves of $\Delta \mathrm{V}_{\mathrm{E}}$ before vs. after $\mathrm{CBD}$ in relation to $\mathrm{PO}_{2}: \mathrm{F}=4.8, p=0.0042$ ).

\section{Histology}

As reported previously (Basting et al., 2015) ArchT-expressing neurons (EYFPimmunoractive) were located at or close to the ventral medullary surface and included TH-ir neurons and neurons devoid of TH immunoreactivity. Both cell types express the transcription factor Phox2b (Basting et al. 2015). The EYFP-immunoreactive TH-negative neurons were defined as RTN neurons i.e. as presumptive central respiratory chemoreceptors (Guyenet \& Bayliss, 2015). Caudally, the TH-positive neurons are C1 cells; rostrally these cells belong to the A5 noradrenergic cluster.

The rostrocaudal distribution of the two populations of Arch-tranduced neurons identified in the 7 rats subjected to CBD and in 6 of the sham-operated rats is shown in Figure 8B. The estimated total number of $\mathrm{ArchT}^{+} \mathrm{RTN}$ neurons per rat (neurons counted in a one-in-six series of sections multiplied by 6 ) was $433.7 \pm 188.6$ (range: $252-750$ ) for the 7 rats subjected to CBD and $751 \pm 221$ (range: 336-924) in the 6 sham-operated rats. The corresponding number of $\mathrm{ArchT}^{+}$catecholaminergic neurons was $399.4 \pm 124.8$ (range: $222-540$ ) in CBD rats and $552 \pm$ 294.9 (range: $264-870$ ) in sham-operated rats. Thus, $44.7 \pm 3.7 \%$ of the ArchT $^{+}$neurons were catecholaminergic and the rest $(55.3 \% \pm 3.4 \%)$ were RTN neurons. The total number of RTN neurons is approximately 2100 per rat (Takakura et al., 2008b), therefore we also estimate that 
$20.7 \pm 8.9 \%$ of RTN neurons expressed ArchT in the 7 rats subjected to CBD and $35.8 \pm 10.5 \%$ of RTN neurons expressed ArchT in the 6 sham-operated rats.

\section{Relationship between number of ArchT-expressing RTN neurons and light-induced hypopnea}

After $\mathrm{CBD}$, the $\mathrm{V}_{\mathrm{E}}$ reduction elicited by ArchT activation was inversely related to the number of ArchT-expressing RTN neurons ( $r^{2}=0.348 ; P=0.0338 ;$ Fig. 8C). The linear correlation between the hypopnea and the number of ArchT-expressing C1 neurons was not statistically significant $\left(r^{2}=0.258 ; P=0.0766 ;\right.$ Fig. $\left.8 D\right)$.

Next, we attempted to estimate the total contribution of RTN neurons to the central chemoreflex as follows. We assumed that the slope of the relationship between ArchT-induced hypopnea (delta $\mathrm{V}_{\mathrm{E}}$ ) and arterial $\mathrm{pH}\left(\right.$ or $\mathrm{PCO}_{2}$, Figure $7 \mathrm{~A}, \mathrm{~B}$ ) represented the instant contribution of the ArchT-transduced RTN neurons to the chemoreflex and we expressed these values as percent of the mean resting $V_{E}$ under normoxia $(30.1 \mathrm{ml} / 100 \mathrm{~g} / \mathrm{min})$. The slope of the relationship was $3.3 \pm 1.3 \%$ resting $\mathrm{V}_{\mathrm{E}} / 0.01$ arterial $\mathrm{pH}$ or $2.4 \pm 0.8 \%$ resting $\mathrm{V}_{\mathrm{E}} / \mathrm{mmHg}$ arterial $\mathrm{PCO}_{2}$. In order to estimate the total contribution of RTN neurons (ArchT-expressing or not) to the reflex we corrected the above values by the mean percentage of RTN neurons that expressed ArchT. The resulting estimate of the contribution of RTN to the chemoreflex was $15.9 \pm 6.7 \%$ resting $\mathrm{V}_{\mathrm{E}} / 0.01$ arterial $\mathrm{pH}$ or $12 \pm 3.9 \%$ resting $\mathrm{V}_{\mathrm{E}} / \mathrm{mmHg}$ arterial $\mathrm{PCO}_{2}$. This estimate was then compared to the slope of the hypercapnic chemoreflex in 4 CBD rats. These rats were exposed to room air supplemented with 0,3 and $6 \% \mathrm{CO}_{2}$ in the plethysmography chamber and their blood gases and ventilation measured after $35 \mathrm{~min}$ exposure to each condition. The gain of 
the hypercapnic ventilatory reflex measured under these conditions was $17.3 \pm 2.4 \%$ resting $V_{E}$ /0.01 arterial $\mathrm{pH}\left(12 \pm 1.72 \%\right.$ resting $\mathrm{V}_{\mathrm{E}} / \mathrm{mmHg}$ arterial $\left.\mathrm{PCO}_{2}\right)$.

\subsection{Discussion}

We report and discuss the following new observations along with confirmatory findings regarding the generation of sighs in hypoxia. Seven days after CBD, blood gases and ventilation in resting rats breathing room air did not differ from pre-CBD. The hypopnea elicited by inhibiting RTN neurons and nearby $\mathrm{C} 1$ cells remains linearly related to arterial $\mathrm{pH}$ and $\mathrm{PCO}_{2}$ after carotid body denervation. RTN/C1 inhibition produces a greater degree of hypopnea after than before $\mathrm{CBD}$, except under hyperoxic conditions.

We interpret the results as follows. The hypopnea produced by combined optogenetic inhibition of $\mathrm{C} 1$ and RTN neurons is probably caused by inhibiting the latter. A week after CBD, the arterial $\mathrm{PCO}_{2}$ set-point of rats breathing room air returns to normal. RTN neurons still encode arterial $\mathrm{pH} / \mathrm{PCO}_{2}$ after $\mathrm{CBD}$ consistent with the notion that these neurons are a nodal point for the regulation of breathing by $\mathrm{CO}_{2}$. RTN neuron inhibition causes a more sustained hypopnea after CBD but this phenomenon can be explained by the loss of the rapid countervailing influence of the carotid bodies without invoking plasticity.

\section{Recovery of normal ventilation and blood gases a week after CBD despite persisting reduction} of the hypoxic ventilatory reflex (HVR)

In intact rats breathing room air, blood gases were as previously reported for quietly resting unstressed rats (Olson et al., 1988a). Weight normalized values for basal ventilation on 
room air were similar to prior published values for the same rat strain (Strohl et al., 1997;

Mouradian et al., 2012). Also as described before, the hypoxic ventilatory reflex of intact rats consisted of a selective increase in breathing frequency (Hodges et al., 2002). The magnitude of the breathing stimulation elicited at $12 \% \mathrm{FiO}_{2}$ in intact rats (average $48.8 \pm 19.1 \%$ increase in $\mathrm{V}_{\mathrm{E}}$ ) was consistent with values reported by prior investigators for the same strain (Hodges et al., 2002). Six to seven days after bilateral CBD, the hypoxic ventilatory reflex was greatly attenuated but not eliminated, also as previously reported (Mouradian et al., 2012). Specifically, there was still a significant overall effect of $\mathrm{FiO}_{2}$ on $F_{R}$ in CBD rats (one-way ANOVA) and $F_{R}$ at $12 \% \mathrm{FiO}_{2}$ was modestly but significantly higher than in room air. The slight plasma alkalization and reduced arterial $\mathrm{PCO}_{2}$ observed in $\mathrm{CBD}$ rats exposed to $12 \% \mathrm{FiO}_{2}$ confirmed the persistence of a small ventilatory stimulation even though hypoxia did not produce a statistically significant increase in $\mathrm{V}_{\mathrm{E}}$ after $\mathrm{CBD}$.

In rats, arterial $\mathrm{PCO}_{2}$ increases immediately after $\mathrm{CBD}$ but eventually returns towards control. This recovery took ten weeks in one study (Olson et al., 1988a), between four and ten days in another (Mouradian et al., 2012). In the present study blood gases in resting rats breathing room air were at control levels seven days after CBD while the hypoxic ventilatory reflex was still greatly depressed. Differences in the surgical procedure used to denervate the carotid bodies may account for the variable recovery kinetics. Here, we purposefully chose to perform our experiments one week after surgery because at later times, the hypoxic ventilatory reflex recovers completely in rats (Curran et al., 2000; Timmers et al., 2003; Teppema \& Dahan, 2010; Hodges \& Forster, 2012; Angelova et al., 2015).

\section{Experimental limitations}


The interpretation of the present optogenetic data relies on four assumptions. The first, which has been verified previously (Basting et al., 2015) is that the ArchT-expressing RTN neurons are silenced by green light and instantly recover their original discharge rate when the light is switched off. The second assumption is that ArchT is only expressed by neurons; this premise has also been verified previously, by histological means (Basting et al., 2015). The third assumption is that, although both RTN and C1 neurons expressed ArchT, the light-induced inhibition of breathing results predominantly from silencing RTN neurons. This assumption is highly plausible but not fully demonstrated. Stimulation of the C1 cells produces some breathing stimulation (Burke et al., 2014) therefore inhibiting these cells could conceivably have the converse effect. However, combined inhibition of ArchT-expressing RTN and C1 cells produces very little blood pressure reduction in quietly resting rats $(<5 \mathrm{mmHg}$; unpublished results by $\mathrm{T}$. Basting, I. Wenker and P. Guyenet) which suggests that the C1 cells have a very low resting discharge under normoxia. C1 cells are robustly activated by hypoxia, however (Reis et al., 1989; Sun \& Reis, 1994; Sun et al., 1996; Reis et al., 1997; Paton et al., 2009; Teppema \& Dahan, 2010). If the breathing response caused by inhibiting a mixed population of C1 and RTN neurons had been predominantly caused by $\mathrm{C} 1$ cell inhibition one would have expected the strongest inhibition of breathing to have occurred under hypoxic conditions, however the exact opposite was observed ((Basting et al., 2015), present study). Thus, in the present experiments, RTN inhibition was very likely primarily responsible for the observed breathing reduction. This interpretation is supported by the tight correlation between the degree of hypopnea elicited by inhibiting ArchT+ neurons with the number of RTN rather than $\mathrm{C} 1$ cells.

\section{RTN inhibition reduces breathing frequency after CBD}


Consistent with prior reports, we observed that hypoxia stimulates predominantly breathing frequency in rats and that this stimulation requires intact carotid bodies (Roux et al., 2000; Coles et al., 2002; Mouradian et al., 2012). Several reports state that central respiratory chemoreceptors regulate tidal volume selectively (Nattie et al., 2001; Wakai et al., 2015). In unaesthetized rodents at least, this is clearly not the case. RTN stimulation or hyperoxic hypercapnia (to activate preferentially central chemoreceptors) produce large increases in breathing frequency in quietly awake rats and mice habituated to their environment or even asleep (Abbott et al., 2011; Holloway et al., 2015; Kumar et al., 2015). Indeed, before birth, selective stimulation of RTN neurons increases breathing frequency in preparations that are devoid of carotid body input (Onimaru \& Homma, 2003; Ruffault et al., 2015). Furthermore, even in the absence of carotid body input, breathing frequency is still robustly increased by hypercapnia in rats, optogenetic inhibition of RTN neurons continues to reduce breathing frequency and, more importantly, this frequency reduction is still proportional to arterial $\mathrm{pH}$ or arterial $\mathrm{PCO}_{2}$ (this study). The latter characteristic strongly suggests that the frequency regulating RTN neurons are activated by $\mathrm{CO}_{2}$. Whether these particular RTN neurons are directly chemosensitive (Guyenet \& Bayliss, 2015; Kumar et al., 2015) or activated by brain $\mathrm{PCO}_{2}$ via synaptic inputs and surrounding astrocytes (Gourine et al., 2010; Nattie, 2012) or both is not addressed by the present results.

\section{Breathing does not require RTN neurons to be active}

In intact rats exposed to hypocapnic hypoxia, the breathing reduction (both $F_{R}$ and $V_{T}$ ) elicited by optogenetic inhibition of RTN neurons is inversely correlated with the $\mathrm{FiO}_{2}$ and 
greatly reduced at $12 \% \mathrm{FiO}_{2}$ (Basting et al., 2015). These results were replicated in the present study. Ventral surface cooling in awake goats produces somewhat less hypopnea (10\%) when the animals breathe a hypoxic vs. hypercapnic mixture suggesting that the hypoventilation could have partly resulted from RTN neuron inhibition (Pan et al., 1995). In rats, the breathing inhibition elicited by optogenetic inhibition of RTN is restored if the plasma is reacidified, suggesting that hypoxia probably inhibits RTN neurons via respiratory alkalosis, i.e. by removing the direct or indirect stimulatory effect of $\mathrm{CO}_{2}$ on these neurons (Gesell et al., 1940; Ainslie \& Duffin, 2009; Basting et al., 2015). Finally hypoxic stimulation of the carotid bodies can compensate for a reduction in activity of the medullary respiratory centers in awake goats (Pan et al., 1995). Collectively, these results suggest that, under hypoxia, breathing is normally sustained by inputs from both CBs and central chemoreceptors and that, when peripheral chemoreceptors are sufficiently stimulated, breathing does not require RTN neurons to be active and, perhaps by extension, that no central chemoreceptor needs to be active. This interpretation is also congruent with the results of Fiamma et al. (2013) obtained in an arterially perfused rat preparation; in this model, fictive breathing could still be maintained by carotid body stimulation when the brain was subjected to a degree of hypocapnia so severe that, theoretically, central chemoreceptors should have been inactivated. Finally, the selective genetic loss of RTN is not lethal in mice; the animals' survival probably relies on a ventilatory stimulus that originates in the carotid bodies (Ramanantsoa et al., 2011).

\section{Central chemoreflex plasticity after carotid body denervation: does the phenomenon exist in rats, do RTN neurons contribute?}


Carotid body plasticity is a well documented phenomenon (Kumar \& Prabhakar, 2012; McBryde et al., 2013; Nurse, 2014). By contrast, central chemoreceptor plasticity is more elusive because, at present, this notion is based on interpretations of changes in the threshold and the gain of the hypercapnic ventilatory reflex rather than on direct measurement of the properties of central chemoreceptors (Hodges \& Forster, 2012). The principal evidence for central chemoreceptor plasticity is that, some time after CBD, (around a week in rats), resting ventilation and blood gases return to normal (in normoxia) despite a severe persisting reduction of the peripheral chemoreflex (Mouradian et al., 2012). The question raised in the present study is whether this return to the status quo is a neuroplasticity phenomenon and whether the RTN is responsible. Specifically, are the carotid bodies tonically active in normal quiescent rats breathing room air and does RTN hyperactivity compensate for the loss of this tonic input after $\mathrm{CBD}$ ?

At first blush, this interpretation seems well supported by the results; indeed, seven days after $\mathrm{CBD}$, blood gases and ventilation in $21 \% \mathrm{O}_{2}$ were at control pre-CBD levels and the hypopnea elicited by inhibiting RTN neurons for $10 \mathrm{~s}$ was significantly larger than before CBD suggesting that breathing was maintained at control levels by an increased excitatory drive from RTN. However, this interpretation is less convincing when the response kinetics are scrutinized. Specifically, the immediate hypopnea (first few seconds) elicited by RTN inhibition in rats breathing room air was very similar before and after CBD (Figs. 4 and 6). The difference was that, in intact rats, the inhibition was less sustained. The simplest explanation for the return of breathing towards control while the laser was still on is that the initial hypopnea activated the peripheral chemoreceptors within this short time frame and this activation gradually reduced the hypopnea elicited by optogenetic inhibition of RTN. Consistent with this interpretation, 
breathing did not recover during ArchT photoactivation in intact rats exposed to $65 \% \mathrm{FiO}_{2}$ or in CBD rats under any oxygen condition. The slow time constant of the response of central chemoreceptors to changes in arterial $\mathrm{PCO}_{2}$ likely explains why ventilation stayed constant throughout the period of RTN inhibition (Fatemian et al., 2003; Smith et al., 2006).

One explanation of the return of breathing and arterial $\mathrm{PCO}_{2}$ to control levels a week after CBD could be that in resting rodents, unlike in larger mammals, the CBs do not contribute significantly to resting ventilation in normoxia; therefore, the absence of the carotid bodies would make no difference, also at rest, once the animals are fully healed. Further support for this hypothesis is that hyperoxia, which is assumed to silence the carotid bodies, produces very little hypopnea in intact rodents (present results and (Olson et al., 1988a; Basting et al., 2015)). However, if this hypothesis is true, some explanation is required for the transient period of hypopnea and hypercapnia that follows CBD (Olson et al., 1988a; Mouradian et al., 2012). One possibility is that surgical deafferentation is not equivalent to merely silencing the CBs. CBD increases the baroreflex gain despite collateral damage to carotid baroreceptors (Del Rio et al., 2013; McBryde et al., 2013), may damage the sympathetic innervation of the CNS vasculature and likely causes temporary inflammation and synaptic rearrangement within the nucleus of the solitary tract with unpredictable consequences on metabolism and the respiratory system. A non-surgical method capable of quickly, reversibly and selectively inhibiting the carotid bodies of intact animals will be required in order to measure accurately the contribution of the CBs to breathing under normoxia in fully intact mammals. We speculate that the CBs of rats might have a slightly higher discharge threshold to arterial $\mathrm{PO}_{2}$ and or $\mathrm{pH}$ than that of larger species (goats, dogs, humans). Such a difference could explain the variablilty of the contribution of the CBs vs. central chemoreceptors to resting ventilation under normoxia. Given the apparent absence of 
plasticity of $\mathrm{CO}_{2}$-responsive central chemoreceptors (rats, this study), a slight change in $\mathrm{CB}$ sensory transduction could also in theory explain why CBD produces such a long-lasting hypoventilation and rise in $\mathrm{PCO}_{2}$ set point in large species and not in rats (Forster, 2003; Miller et al., 2013; Angelova et al., 2015). A major species difference in the central circuitry underlying the chemoreflexes need not be invoked to explain these observations.

In sum, the loss of the peripheral chemoreflex could be sufficient to explain the increased hypopnea elicited by inhibiting RTN neurons seven days after CBD in rats breathing room air or a hypoxic mixture. The carotid body afferents of normal quietly resting rats breathing room air may be silent and the temporary breathing changes that follow $C B$ denervation may have other causes than the mere silencing of these sensory afferents. We acknowledge that, in goats, in which CBD produces an extremely long-lasting hypopnea, neurochemical changes which could, at least theoretically, underlie these persistent changes in breathing, have been observed in the brainstem (Pan et al., 1998; Miller et al., 2013). We did not find clear evidence of RTN plasticity in rats a week after CBD but the contribution of the carotid bodies to resting ventilation could be highly species-dependent and it is also possible that following $\mathrm{CBD}$, adaptive changes in respiratory circuitry are species-specific or occur somewhere else than in RTN.

\section{Long-lasting breathing inhibition in response to RTN inhibition}

As discussed above, the kinetics of the breathing response produced by RTN inhibition varied depending on the prevailing $\mathrm{FiO}_{2}$ and on whether the carotid bodies were intact or denervated. The breathing responses observed under these conditions also differed during the recovery phase. In hyperoxia (intact rats) or indeed under all oxygen conditions in CBD rats, 
breathing returned relatively slowly to control after the light was switched off ( $T_{1 / 2}$ of approximately 20 s). This feature is counterintuitive; when the light was switched off, a ventilation overshoot would have been expected because of the preceding hypopnea and resulting $\mathrm{CO}_{2}$ accumulation. The slow recovery is probably not caused by a protracted inhibition of RTN neurons because, at least in anesthetized rats, the discharge rate of these neurons returns instantly to control at the end of light induced inhibition (Basting et al., 2015). In addition, a similarly protracted ventilatory response $\left(T_{1 / 2}: 12.4 \mathrm{~s}\right)$ but of opposite sign is observed in anesthetized ventilated rats when RTN neurons are activated with channelrhodopsin (Abbott et al., 2009a). RTN neurons instantly recover their initial discharge rate after stimulation with channelrhodopsin, therefore the long-lasting ventilatory stimulation is not caused by a persistent increase in the discharge rate of these neurons (Abbott et al., 2009a). In sum, the persistence of the ventilatory effects observed after a short period of excitation or inhibition of RTN neurons probably reflect the cellular and integrative properties of the respiratory rhythm and pattern generating network downstream from RTN neurons. Alternately, this phenomenon could result from the release of a slow transmitter by RTN neurons (Stornetta et al., 2009).

\section{Linear relationship between RTN activity and $\mathrm{pHa} / \mathrm{PCO}_{2}$ persists after CBD}

In the following section, RTN "activity" refers to the contribution of ArchT-expressing RTN neurons to ventilation as measured by the breathing reduction elicited when these neurons are photoinhibited. As illustrated in Figure 7, the activity of RTN neurons remained a linear function of arterial $\mathrm{pH}, \mathrm{PCO}_{2}$ and $\left[\mathrm{HCO}^{-}\right]$after $\mathrm{CBD}$. By linear relationship we mean that the data is statistically compatible with such a linear relationship. Although the chemodenervation 
meant that the range of $\mathrm{pH}$ and $\mathrm{PCO}_{2}$ elicited by hypoxia or $3 \% \mathrm{FiCO}_{2}$ was much reduced $(0.06$ $\mathrm{pH} ; 8.5 \mathrm{mmHg} \mathrm{PCO}_{2}$ ), the correlation coefficients between light-induced hypopnea (delta $\mathrm{F}_{\mathrm{R}}$ and delta $\left.\mathrm{V}_{\mathrm{E}}\right)$ and blood gases $\left(\mathrm{pH}, \mathrm{PCO}_{2}\right)$ or $\mathrm{HCO}^{-}$were still high. The slope of these relationships was always slightly greater after $\mathrm{CBD}$ although the change did not reach statistical significance. The trend towards a steeper slope is presumably related to the underestimation of the "activity" of RTN neurons in intact rats because of the countervailing effect of the peripheral chemoreflex as discussed above. After CBD, the slope of this relationship was on average $3.3 \pm 1.3 \%$ of resting $V_{E}$ per $0.01 \mathrm{pH}$ (average resting $V_{E}=31 \mathrm{ml} / \mathrm{min} / 100 \mathrm{~g}$ ). Based on the assumption that the breathing inhibition caused by photoinhibiting RTN is proportional to the fraction of the neurons that express ArchT and only $\sim 20 \%$ of total RTN neurons in the present experiment expressed ArchT, we speculate that the entire nucleus could potentially elicit a change in ventilation equal to $15.9 \%$ resting $\mathrm{V}_{\mathrm{E}} / 0.01$ arterial $\mathrm{pH}$ or $12 \%$ resting $\mathrm{V}_{\mathrm{E}} / \mathrm{mmHg}$ arterial $\mathrm{PCO}_{2}$. These prorated values are almost identical to the average gain of the hypercapnic ventilatory reflex measured in $4 \mathrm{CBD}$ rats of the same strain $\left(17.3 \%\right.$ resting $\mathrm{V}_{\mathrm{E}} / 0.01 \mathrm{pHa}$ or $12 \%$ resting $\mathrm{V}_{\mathrm{E}} / \mathrm{mmHg}$ arterial $\left.\mathrm{PCO}_{2}\right)$. However, the statistical power was inadequate to rule out a type II error (false negative) and we could not use these determinations to assess what percentage of the chemoreflex is mediated by RTN.

In intact rats, a small but consistent decrease in $F_{R}$ and $V_{T}$ in response to photoinhibition of $\mathrm{ArchT}^{+}$neurons persisted even under the most severe respiratory alkalosis $\left(\mathrm{pHa}^{\sim 7.6}, \mathrm{PCO}_{2}\right.$ $\sim 25 \mathrm{mmHg}$; Figure 7). Thus, RTN neurons still retained some activity under such conditions. Under anesthetized normoxic rats or in slices, RTN neurons would be silenced by this degree of alkalosis (Guyenet et al., 2005). However, in the present experiments, the alkalosis was produced by hypoxia. The residual activity of these neurons is likely to have resulted from the 
robust excitatory input that RTN neurons receive from the carotid bodies (Takakura et al., 2006). After $\mathrm{CBD}$, the slopes of the relationship between $\mathrm{F}_{\mathrm{R}}$ (or $\mathrm{V}_{\mathrm{T}}$ ) and arterial $\mathrm{PCO}_{2}($ or $\mathrm{pH}$ ) was steeper (Figure 7) and their intersect with the zero line occurred at more acidic levels (lower $\mathrm{pH}$, higher $\left.\mathrm{PCO}_{2}\right)$. This observation suggests that, in the absence of $\mathrm{CBs}$, a lesser degree of alkalosis is likely required to silence RTN neurons; this is precisely what would be expected from the loss of the excitatory input from the carotid bodies to RTN neurons. Yet, while plausible, the above interpretations are tentative. At least two alternative explanations of the residual activity of RTN neurons during hypoxia induced alkalosis can be invoked. The first is that a fraction of RTN neurons may be only moderately $\mathrm{pH}$-sensitive. Alternately, some fraction of the hypopnea observed under hypoxic conditions was caused by $\mathrm{C} 1$ neuron inhibition. The first possibility is supported by evidence from RTN neurons recording in slices (Lazarenko et al., 2009), the second that $\mathrm{C} 1$ cells (mice and rats) are activated by hypoxia and that their selective activation produces some hyperpnea (Sun \& Reis, 1993; Abbott et al., 2013c; Burke et al., 2014).

\section{Hyperbolic relationship between RTN activity and $\mathrm{PO}_{2}$}

Not surprisingly, the relationship between delta $\mathrm{F}_{\mathrm{R}}$ or delta $\mathrm{V}_{\mathrm{E}}$ and arterial $\mathrm{PO}_{2}$ was hyperbolic and significantly left- shifted after CBD. This shift is consistent with the observation that, for any given level of $\mathrm{PO}_{2}$ below room air, $\mathrm{PCO}_{2}$ is lower in intact than in $\mathrm{CBD}$ rats therefore the resting activity of RTN neurons is also lower and photoinhibition of these neurons produces less hypopnea.

\section{CBD and hypoxia-induced sighs}

Sighs (augmented breaths) may result from a brief reconfiguration of the preBötzinger complex circuitry (Lieske et al., 2000; Ramirez, 2014). Sigh incidence is markedly increased by 
hypoxia. This effect requires intact carotid bodies and is attenuated by supplementing the hypoxic breathing mixture with $\mathrm{CO}_{2}$ or by inducing metabolic acidosis (Bartlett, 1971; Bell et al., 2009; Bell \& Haouzi, 2010). After CBD, hypoxia no longer increased sigh frequency in our experimental animals. We take this result as further evidence that we successfully eliminated the carotid bodies. We also confirmed the results of Haouzi's group (Bell et al., 2009; Bell \& Haouzi, 2010) that, in intact rats exposed to hypoxia, sighing is eliminated by adding $\mathrm{CO}_{2}$ to the breathing mixture. The addition of a mere $3 \% \mathrm{FiCO}_{2}$ returned sigh frequency to the baseline level found in normoxia. Sigh frequency in $12 \% \mathrm{O}_{2}$ supplemented with $3 \% \mathrm{CO}_{2}$ was significantly below the level observed under $15 \% \mathrm{O}_{2}$. However, blood gas analysis showed that the addition of $3 \% \mathrm{CO}_{2}$ to $12 \% \mathrm{FiO}_{2}$ caused arterial $\mathrm{PO}_{2}$ to rise slightly above the level observed in animals exposed to a $15 \% \mathrm{FiO}_{2}$. Therefore, under our experimental conditions, the addition of $\mathrm{CO}_{2}$ could have eliminated sighs simply by reducing carotid body stimulation but alternative interpretations, considered next, are also possible.

Sigh-like events are elicited by hypoxia in brain slices (Lieske et al., 2000; Ramirez, 2014) but, in unaesthetized rats subjected to CBD, hypoxia no longer triggers sighs. The latter in vivo findings argue against a role of CNS hypoxia in triggering sighs but the interpretation of these findings is predicated on the assumption that, in unanesthetized animals exposed to a given low $\mathrm{FiO}_{2}$, brainstem parenchymal $\mathrm{PO}_{2}$ is necessarily lower after $\mathrm{CBD}$ than in intact animals. This could be wrong. In $12 \%$ hypoxia, arterial $\mathrm{PCO}_{2}$ is substantially higher in CBD rats that in intact rats (11 $\mathrm{mmHg}$ in the present case). Because hypercapnia causes cerebral vasodilation, brainstem $\mathrm{PO}_{2}$ could conceivably be higher after CBD despite the slightly reduced arterial $\mathrm{PO}_{2}$. This possibility would also explain why the addition of a small amount of $\mathrm{CO}_{2}\left(3 \% \mathrm{FiCO}_{2}\right)$ suppresses the sighs ((Bell \& Haouzi, 2010); present results). In sum, the suppression of sighs by 
CBD does not eliminate the possibility that brainstem hypoxia could contribute to sigh generation in intact unaesthetized rodents. In an intact animal, the sigh generating circuitry could operate as a coincidence detector requiring both a high level of carotid body activity plus some degree of brainstem hypoxia. Finally, as shown here, hypoxia does not silence RTN neurons in CBD rats. Complete RTN inhibition could conceivably be required for carotid body stimulation to produce sighs. This possibility would also explain why hypoxia-induced sighs are suppressed by hypercapnia or metabolic acidosis.

\section{Summary and conclusions}

A week after CBD, the HVR was greatly attenuated, sighs were no longer elicited by hypoxia and blood gases and resting $V_{E}$ were normal although $F_{R}$ was marginally reduced and $V_{T}$ marginally increased. RTN inhibition produced greater breathing inhibition after than before $\mathrm{CBD}$ but this difference was not observed in hyperoxic conditions and could be explained by the absence of the rapid countervailing influence of the peripheral chemoreflex. Accordingly, we did not find compelling evidence that CBD modifies RTN function and, by extrapolation, central respiratory chemoreception.

The hypopnea elicited by RTN, especially the reduction of breathing frequency and minute ventilation, remains linearly and highly correlated with arterial $\mathrm{PCO}_{2}$ and $\mathrm{pH}$ after $\mathrm{CBD}$. These observations further strengthen the notion that RTN neurons are central respiratory chemoreceptors or, at the very least, operate as a nodal point for the regulation of breathing by central chemoreceptors. Finally, in conscious rats, RTN and, by extension, central chemoreceptors regulate both the frequency and the amplitude of breathing. 
Table 1: Blood parameters in intact rats and in carotid body-denervated rats

\begin{tabular}{|c|c|c|c|c|}
\hline $\mathrm{FiO}_{2} / \mathrm{FiCO}_{2}$ & $\mathrm{pH}$ & $\mathrm{PCO}_{2}$ & $\mathrm{PO}_{2}$ & $\mathrm{HCO}_{3}$ \\
\hline \multicolumn{5}{|l|}{ Intact rats $(\mathrm{N}=6)$} \\
\hline $65 \%$ / 0\% & $7.4 \pm 0.03 *$ & $49.2 \pm 5.7$ & $270.8 \pm 20 * * *$ & $30.4 \pm 2.7$ \\
\hline $21 \%$ / 0\% & $7.44 \pm 0.02$ & $41.8 \pm 4.7$ & $78.3 \pm 7.4$ & $29 \pm 1.8$ \\
\hline $15 \% / 0 \%$ & $7.51 \pm 0.02 * * *$ & $32.6 \pm 2.2^{*}$ & $51.5 \pm 6.3^{* * *}$ & $26.1 \pm 1.9 *$ \\
\hline $12 \% / 0 \%$ & $7.59 \pm 0.03 * * *$ & $25 \pm 1.4^{* * *}$ & $43 \pm 5.9^{* * *}$ & $23.9 \pm 2.2 * * *$ \\
\hline $12 \% / 3 \%$ & $7.48 \pm 0.04 * *$ & $34.5 \pm 2.03$ & $54.6 \pm 7.4^{* * *}$ & $26 \pm 3.2$ \\
\hline \multicolumn{5}{|l|}{ CBD rats $(\mathrm{N}=6)$} \\
\hline $65 \%$ / 0\% & $7.45 \pm 0.01$ & $44 \pm 4.1$ & $270.5 \pm 20.8^{* * *}$ & $30.6 \pm 2.3$ \\
\hline $21 \% / 0 \%$ & $7.44 \pm 0.02$ & $42.7 \pm 5.1$ & $79.3 \pm 10.4$ & $28.9 \pm 2.9$ \\
\hline $18 \% / 0 \%$ & $7.43 \pm 0.01$ & $43.78 \pm 4.7$ & $60 \pm 6.1^{* *}$ & $28.9 \pm 2.9$ \\
\hline $15 \% / 0 \%$ & $7.46 \pm 0.02$ & $39.8 \pm 3.5$ & $45.7 \pm 5.3^{* * *}$ & $27.8 \pm 2.3$ \\
\hline $12 \% / 0 \%$ & $7.49 \pm 0.03$ & $35.5 \pm 3.1^{* * *}$ & $34 \pm 4.4^{* * *}$ & $27.5 \pm 3^{*}$ \\
\hline $12 \%$ / 3\% & $7.43 \pm 0.01$ & $42 \pm 4.6$ & $48.8 \pm 5.8^{* * *}$ & $27.6 \pm 2.7$ \\
\hline
\end{tabular}

All values are compared to those found in rats exposed to $21 \% \mathrm{FiO}_{2} .{ }^{*} p<0.05$;

${ }^{* *} p<0.01 ;{ }^{* *} p<0.005$; one-way RM ANOVA followed by Dunnett's multiple comparisons test. 
Table 2: hypercapnic ventilatory reflex 7 days after CBD

\begin{tabular}{|c|c|c|c|c|c|c|c|}
\hline $\mathrm{FiCO}_{\mathbf{2}}$ & $\mathbf{p H}$ & $\mathbf{P C O}_{\mathbf{2}}$ & $\mathbf{P O}_{\mathbf{2}}$ & $\mathbf{H C O}_{3}$ & $\mathbf{F}_{\mathrm{R}}$ & $\mathbf{V}_{\mathbf{T}}$ & $\mathbf{V}_{\mathrm{E}}$ \\
\hline & 7.4 & 47.4 & 77.8 & 29.5 & 78.5 & 0.38 & 29.8 \\
$\mathbf{0 \%}$ & \pm 0.02 & \pm 2.4 & \pm 10.4 & \pm 0.8 & \pm 15.9 & \pm 0.04 & \pm 5.3 \\
\hline & 7.38 & 50.5 & 100.8 & 30.1 & 102 & 0.49 & 50.3 \\
$\mathbf{3 \%}$ & $\pm 0.03^{* *}$ & $\pm 2.9 *$ & $\pm 5.3^{*}$ & \pm 1.2 & \pm 17.2 & \pm 0.09 & $\pm 9.8^{* *}$ \\
\hline & 7.33 & 58.9 & 124.3 & 30.8 & 136 & 0.53 & 72.1 \\
$\mathbf{6 \%}$ & $\pm 0.02^{* *}$ & $\pm 2.6^{* * *}$ & $\pm 16.4^{* *}$ & \pm 0.8 & $\pm 11.2^{* *}$ & $\pm 0.03^{* *}$ & $\pm 6.1^{* * *}$ \\
\hline
\end{tabular}

$\mathrm{CBD}$ rats $(\mathrm{N}=4)$ were exposed to room air $\left(21 \% \mathrm{FiO}_{2}\right)$ supplemented with various levels of $\mathrm{FiCO}_{2}$ $(0,3$ or $6 \%)$. Breathing and blood parameters shown as mean \pm SD. ${ }^{*} p<0.05$; ${ }^{*} p<0.01$; *** $p<0.005$; one-way RM ANOVA followed by Dunnett's multiple comparisons test. 


\subsection{Figures}

Figure 1.

A

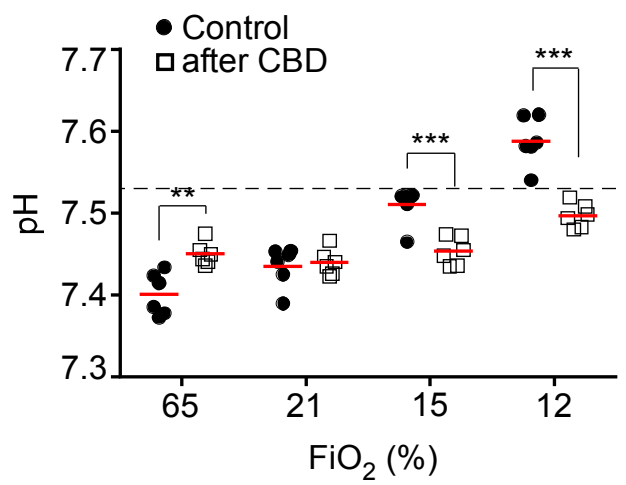

C

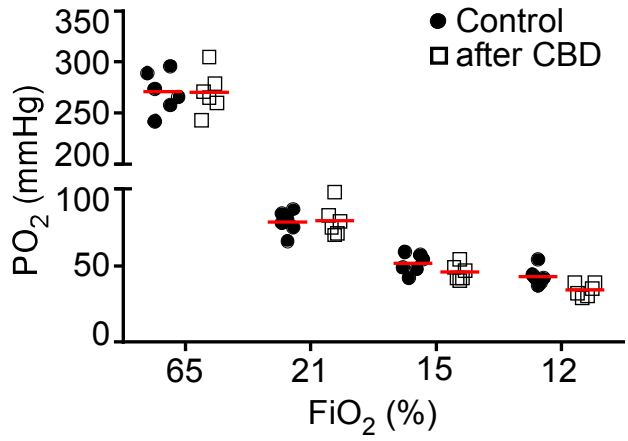

B

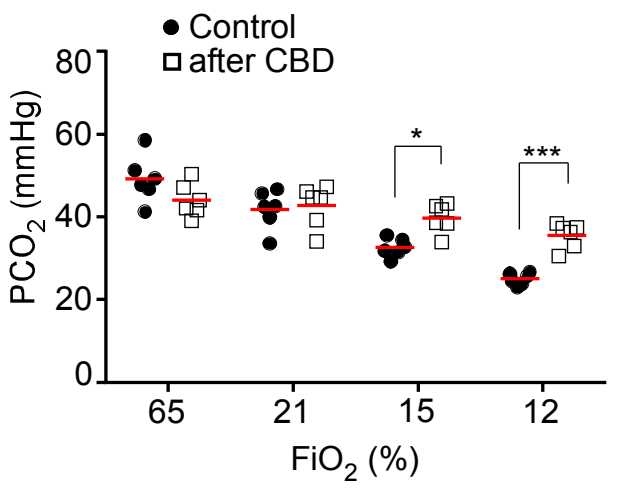

D

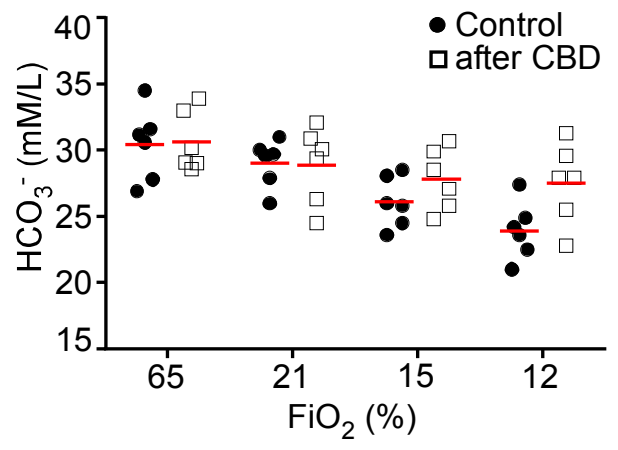


Figure 1. Arterial blood parameters at four levels of $\mathrm{FiO}_{2}$ in intact vs. carotid body denervated (CBD) rats.

A, arterial pH vs. $\mathrm{FiO}_{2}$. B, arterial $\mathrm{PCO}_{2}$ vs. $\mathrm{FiO}_{2}$. C, arterial $\mathrm{pO}_{2}$ vs. $\mathrm{FiO}_{2}$. D, arterial $\left[\mathrm{HCO}_{3}\right]$ vs. $\mathrm{FiO}_{2}$. Control: $\mathrm{N}=6$; CBD: $\mathrm{N}=7$ ). ${ }^{*} \mathrm{P} \leq 0.05 ;{ }^{* *} \mathrm{P} \leq 0.01 ; * * \mathrm{P} \leq 0.001$ (2-way ANOVA). 
Figure 2.

\section{A1}
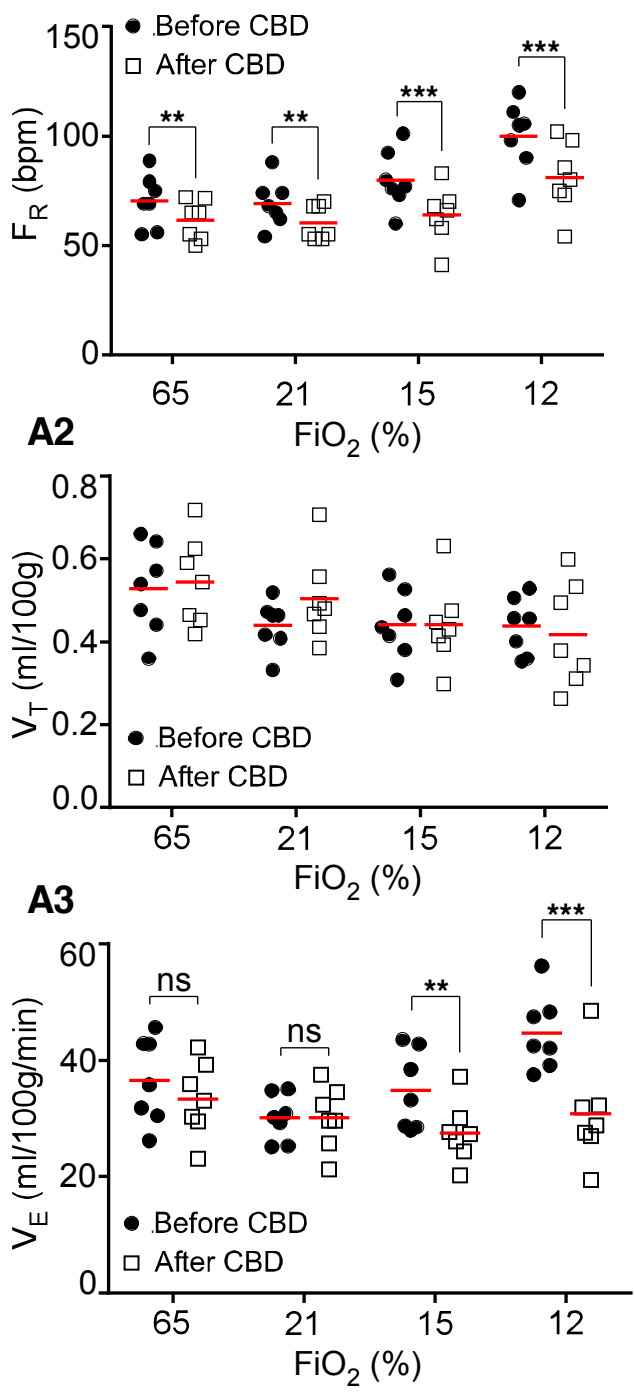
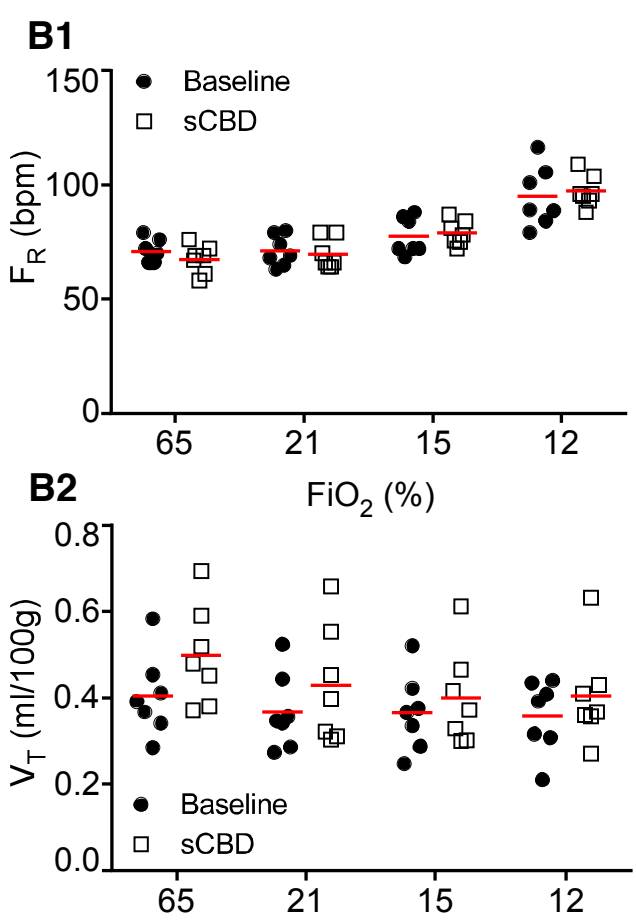

$$
\text { B3 } \quad \mathrm{FiO}_{2}(\%)
$$

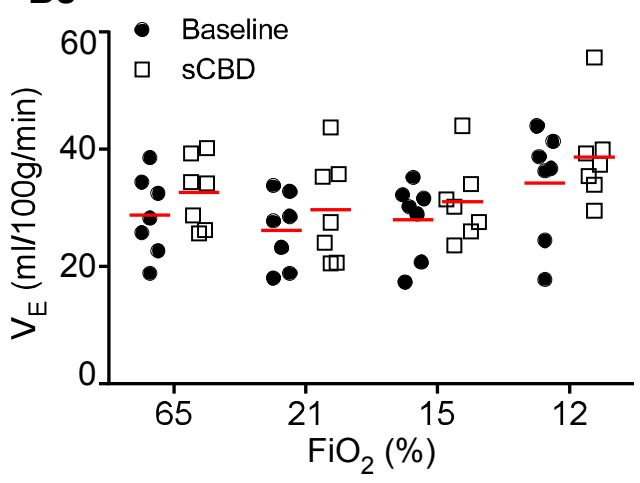


Figure 2. Effect of $\mathrm{FiO}_{2}$ on breathing before and after CBD or sham surgery.

A1-A3: from top to bottom, effect of $\mathrm{FiO}_{2}$ on breathing frequency, tidal volume and minute volume) before and 7 days after $\mathrm{CBD}(\mathrm{N}=7)$. $\mathrm{B} 1-\mathrm{B} 3$, effect of $\mathrm{FiO}_{2}$ on breathing before and 7 days after sham surgery $(\mathrm{N}=7) .{ }^{*} \mathrm{P} \leq 0.05 ;{ }^{* *} \mathrm{P} \leq 0.01 ; * * \mathrm{P} \leq 0.001$ (2-way ANOVA). 


\section{Figure 3.}

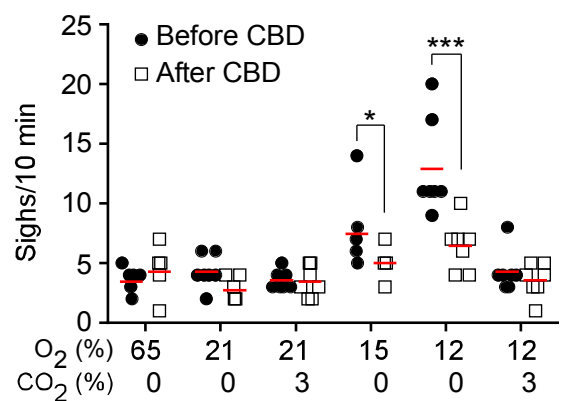


Figure 3. Sighing at four levels of $\mathrm{FiO}_{2}$ in intact vs. carotid body denervated (CBD) rats.

Hypoxia increased sigh frequency before but not after CBD $(N=7)$. Sighs were suppressed by adding $3 \% \mathrm{FiCO}_{2}$. Effect of $\mathrm{CBD}: * \mathrm{P} \leq 0.05 ; * * * \mathrm{P} \leq 0.001$ (2-way ANOVA). 
Figure 4.

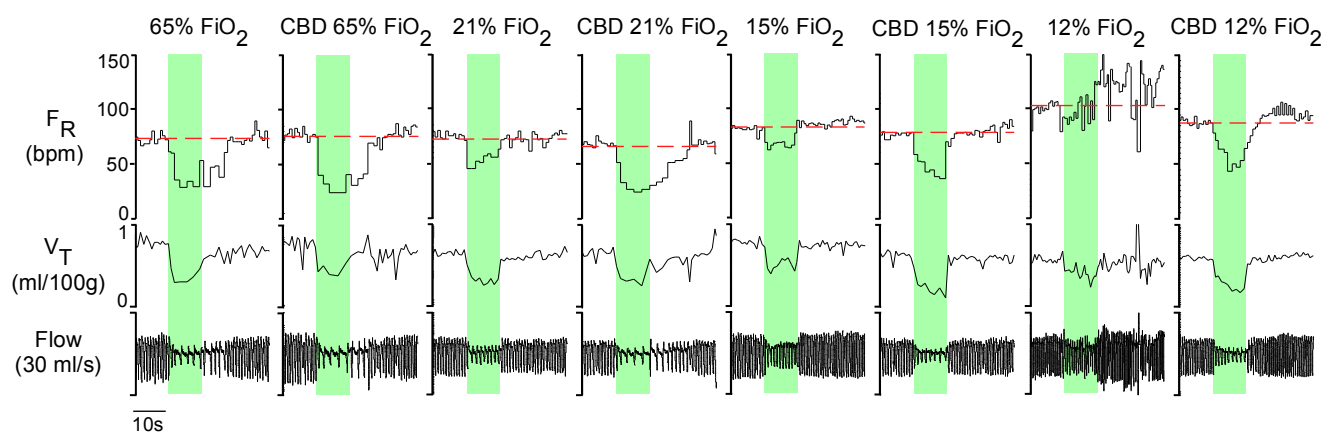


Figure 4. Breathing reduction elicited by bilateral ArchT activation at four $\mathrm{FiO}_{2}$ levels before and after CBD.

Each panel depicts, from top to bottom, breathing frequency, tidal volume and the raw plethysmography trace (airflow velocity). All panels were from the same rat before and fater CBD. Resting $F_{R}$ is indicated by a dashed red line. 
Figure 5.
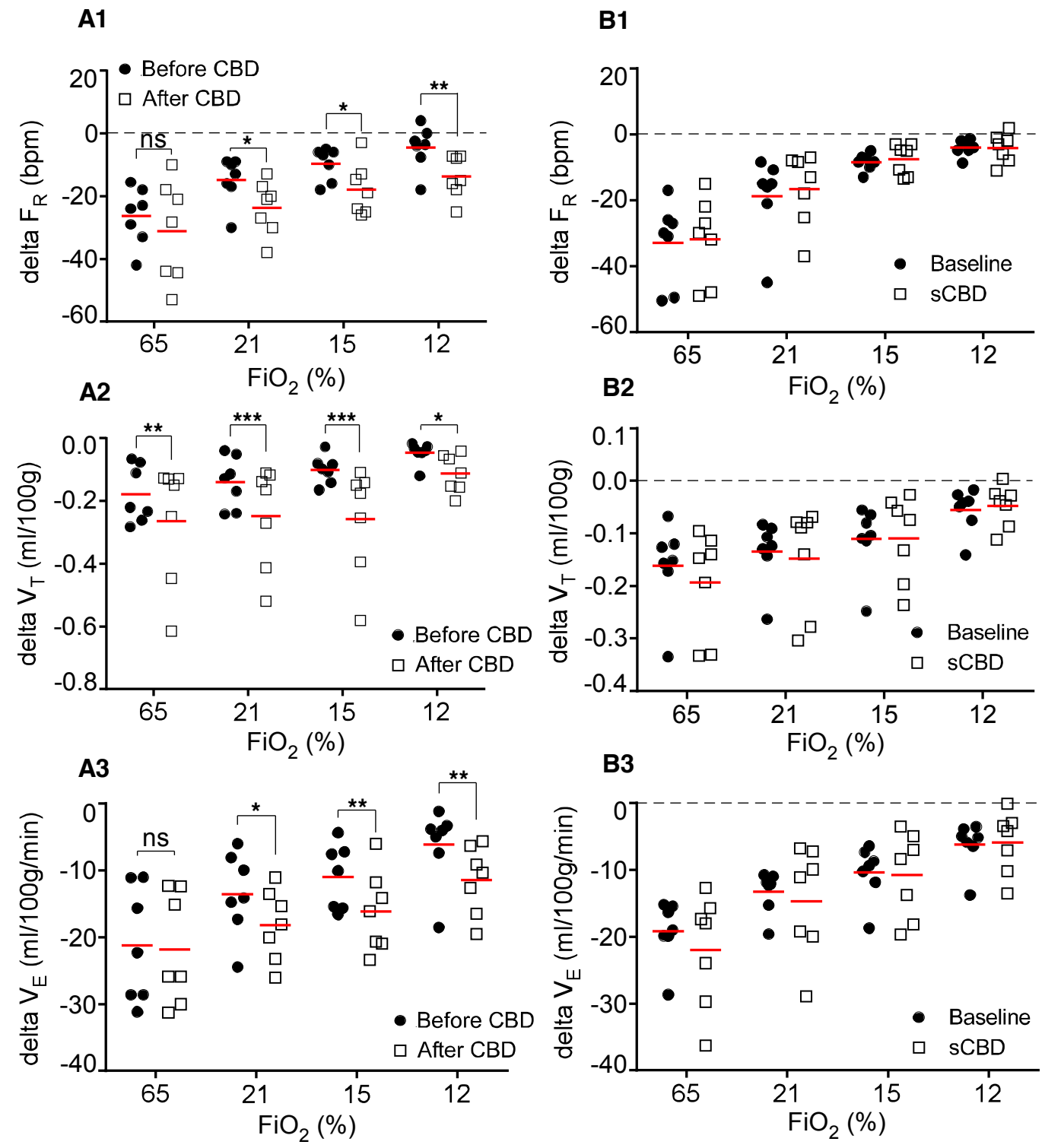
Figure 5. ArchT-induced hypopnea at four levels of $\mathrm{FiO}_{2}$ before and after CBD.

A1-A3: from top to bottom, effect of $\mathrm{FiO}_{2}$ on the reduction in breathing frequency, tidal volume and minute volume elicited by inhibiting ArchT-expressing neurons before and 7 days after CBD $(\mathrm{N}=7)$. B1-B3: identical experiment conducted in an additional 7 rats before and 7 days after sham surgery. ${ }^{*} \mathrm{P} \leq 0.05 ;{ }^{* *} \mathrm{P} \leq 0.01 ; * * * \mathrm{P} \leq 0.001$ (2-way ANOVA). Note that sham surgery had no effect whereas breathing inhibition was enhanced after CBD except under hyperoxia 
Figure 6.
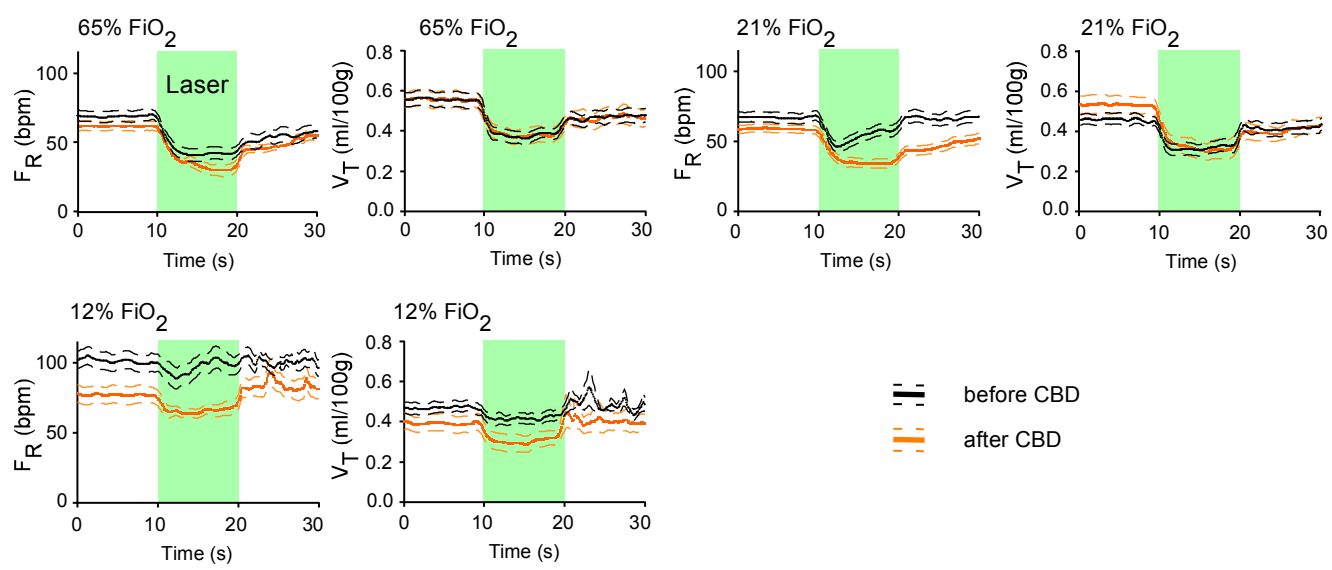

$$
\begin{array}{ll}
\ldots & \text { before } \mathrm{CBD} \\
\ldots & \text { after } \mathrm{CBD}
\end{array}
$$


Figure 6. Kinetics of the hypopnea elicited by bilateral inhibition of ArchT-expressing neurons.

The graphs represent event-triggered averages of the frequency and amplitude components of the breathing response observed at three levels of $\mathrm{FiO}_{2}$ before and after $\mathrm{CBD}$. Four to six responses were collected at each $\mathrm{FiO}_{2}$ level in every rat $(\mathrm{N}=7)$ to generate a single average per rat. Seven such responses were finally averaged. The solid lines represent these grand averages and the dotted lines the $95 \%$ confidence intervals. 
Figure 7 Part 1.

A
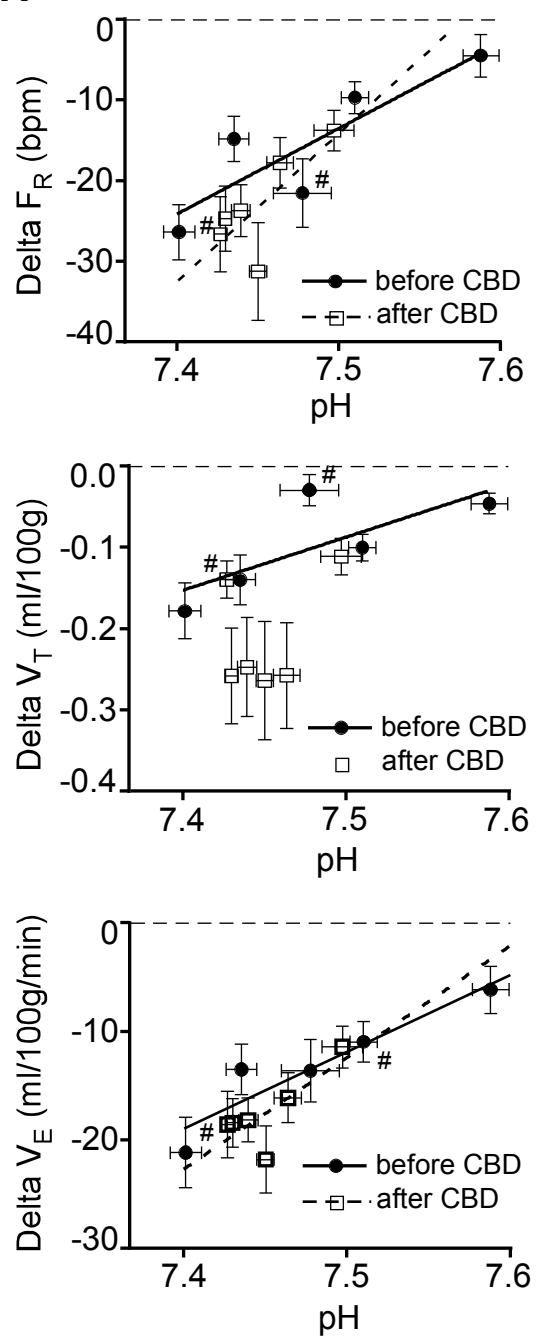

B
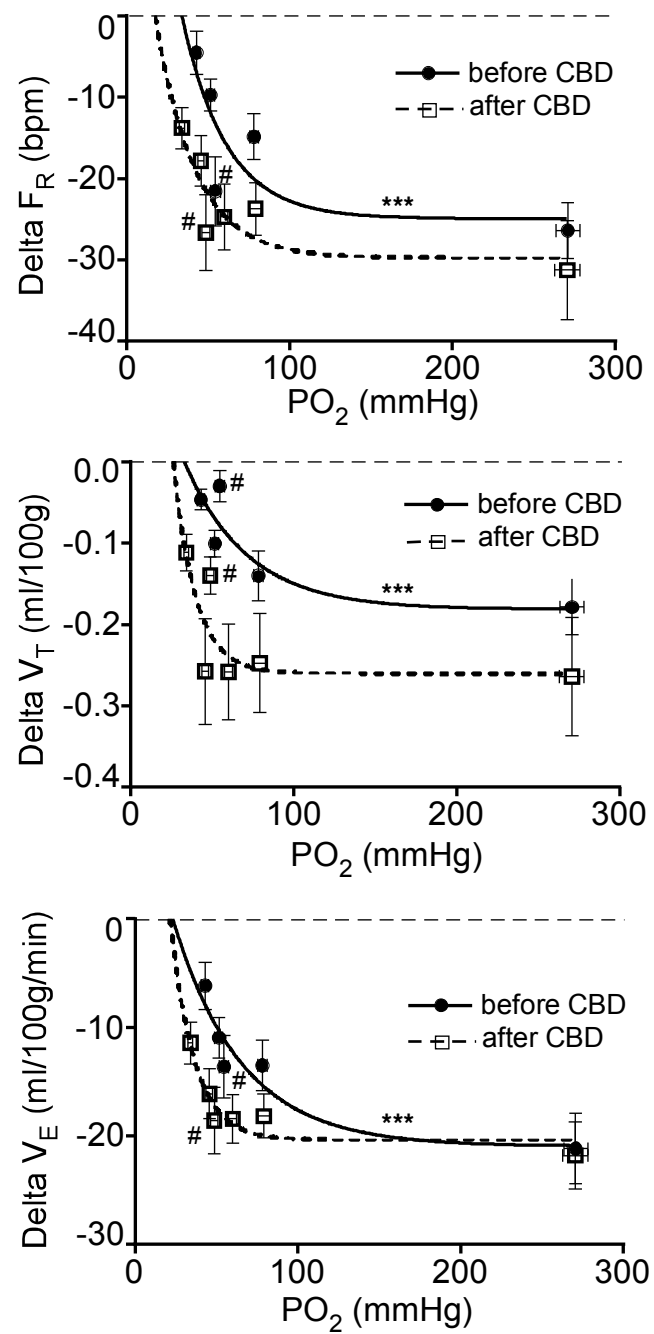
Figure 7 Part 2.
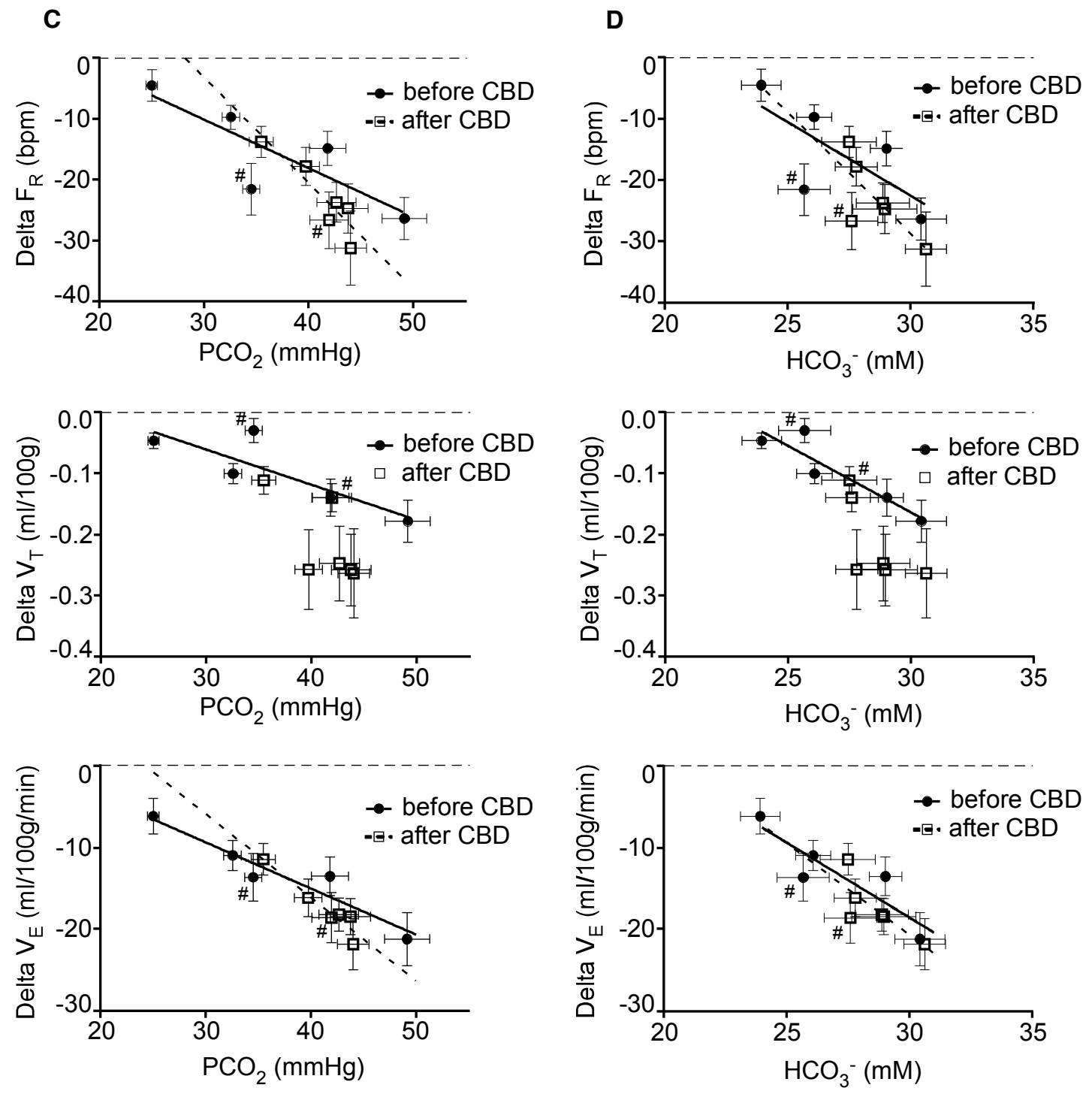
Figure 7. Relationship between ArchT-induced hypopnea and blood gases.

A-D: From top to bottom: reduction of breathing frequency (delta $F_{R}$ ), tidal volume (delta $V_{T}$ ) and minute volume (delta $\mathrm{V}_{\mathrm{E}}$ ) elicited by photostimulating ArchT plotted as a function of arterial $\mathrm{pH}(\mathrm{A})$, arterial $\mathrm{PO}_{2}(\mathrm{~B})$, arterial $\mathrm{PCO}_{2}(\mathrm{C})$ and arterial $\left[\mathrm{HCO}_{3}{ }^{-}\right](\mathrm{D})$. Results (from 6 rats) were obtained under five conditions: $65 \%, 21 \%, 18 \%, 15 \%$ and $12 \% \mathrm{FiO}_{2}$ balance $\mathrm{N}_{2}$ and $12 \% \mathrm{FiO}_{2}$ plus $3 \% \mathrm{FiCO}_{2}$ (the latter condition is indicated by \#). In A, C and D the straight lines indicate that a significant linear correlation between the change in breathing parameter and the blood parameter was identified. In B, the curved lines indicate a difference in the non-linear exponential regression correlation between before and after CBD. 
Figure 8.
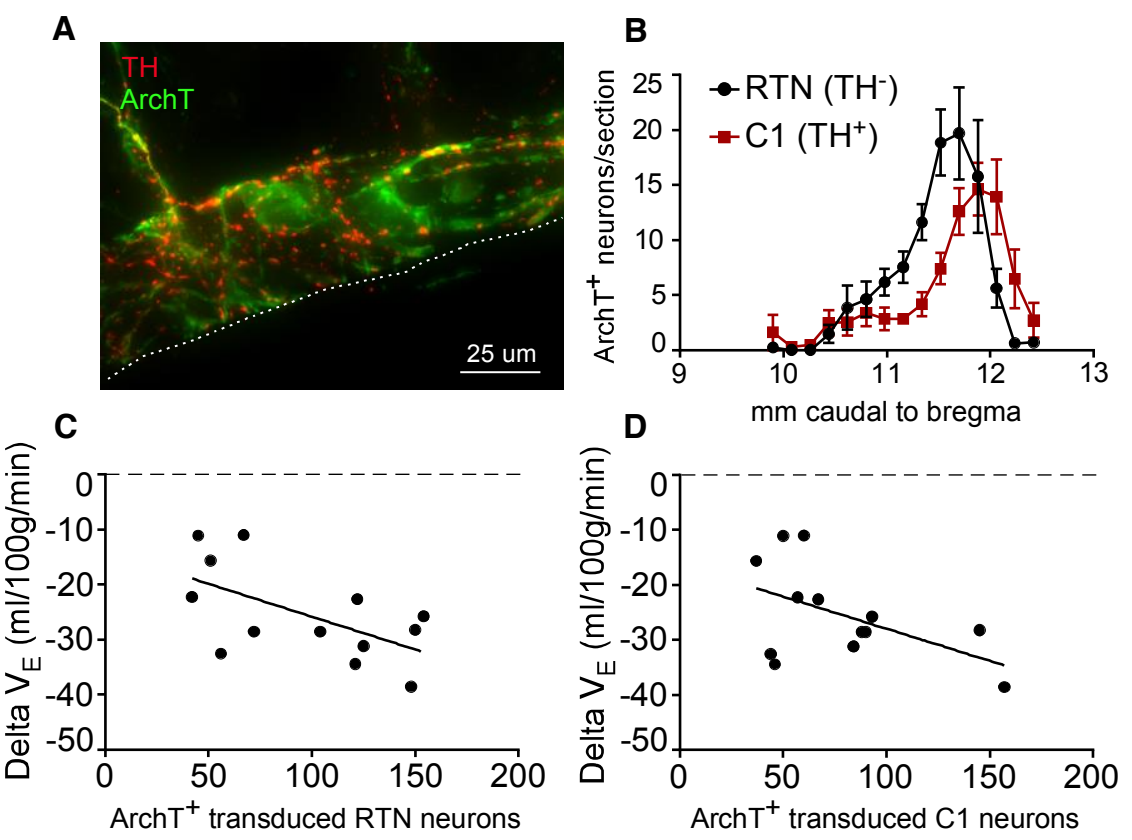
Figure 8. Location and distribution of ArchT-expressing neurons.

A, Coronal section through the medulla (level $~ 11.4 \mathrm{~mm}$ caudal to bregma) illustrating the selective expression of ArchT-EYFP in Phox2b-ir nuclei close to the ventral medullary surface (shown by dashed line). B, Distribution of EYFP-ir neurons after bilateral injections of PRSx8ArchT-EYFP lentiviral vector below the caudal half of the facial motor nucleus (one-in-six series of $30-\mu \mathrm{m}$-thick transverse sections). The plane of section is calibrated after a standard atlas where level $11.6 \mathrm{~mm}$ is defined as the caudal end of the facial motor nucleus (Paxinos \& Watson, 1998). TH-immunoreactive neurons consist mostly of C1 neurons caudally and A5 neurons rostrally. (sections, $180 \mu \mathrm{m}$ apart). C, Relationship between number of all RTN neurons transduced (counted in a 1:6 coronal series) and percentage decrease of $V_{E}$ elicited by Arch photostimulation. D, Relationship between number of all catecholaminergic (C1) neurons transduced and percentage decrease $\mathrm{V}_{\mathrm{E}}$ elicited by Arch photostimulation. ( $\mathrm{N}=13$, combined experimental and sham animals).

Author Contributions: P.G.G., R.L.S., T.M.B. conception and design of research; T.M.B., C.A., K.E.V., performed experiments; T.M.B., R.L.S. analyzed data; T.M.B. prepared figures; P.G.G., T.M.B drafted manuscript; P.G.G., T.M.B, R.L.S. edited and revised manuscript; P.G.G., T.M.B., R.L.S. interpreted results of experiments; P.G.G. approved final version of manuscript. 


\section{Chapter 5: Overview \& Summary}

This dissertation has focused on the contribution of the retrotrapezoid nucleus to breathing automaticity under different oxygen conditions and during various states of vigilance. The interpretations are based on single unit recordings in anesthetized rats and the effect of bilateral RTN optogenetic inhibition on the breathing pattern of conscious rats. This work revealed two essential properties of RTN neurons: first, these neurons are an essential link of the hypercapnic ventilatory reflex (HCVR) in conscious rodents and, second, these neurons are also critical to sustain breathing during sleep. In conjunction with the demonstration by others that RTN neurons encode $\mathrm{PaCO}_{2}$ of the surrounding brain parenchyma, my work has significantly contributed to the view that RTN is a major cluster of central respiratory chemoreceptors and plays a critical role in $\mathrm{PaCO}_{2}$ homeostasis, especially at rest and during non-REM sleep. These findings help understand the pathophysiology of respiratory problems such as central sleep apnea of prematurity, adult central sleep apnea and congenital central hypoventilation syndrome.

\subsection{RTN Drives Breathing}

There has been a great deal of progress made in the past decade in understanding neural control over respiration. Finding the role that RTN neurons play in the drive to breathe has been a challenge wrought with controversy. Li and Nattie found only a moderate increase in $\mathrm{V}_{\mathrm{T}}$ when $\mathrm{CO}_{2}$ is added to this area through microdialysis (Li \& Nattie, 2002) and Marina et al. (2010) reported that inhibition of the RTN makes no difference on baseline respiration in a reduced preparation. The microdialysis technique produced small changes in ventilation relative to that evoked by hypercapnia. This could be due to only a small portion of the respiratory 
network being acidified from the dialysis probe (semipermeable tip diameter $=0.5 \mathrm{~mm}$ ). The major limitation of this approach is the degree of acidification caused by the hypercapnic fluid in the probe. PH electrodes placed "in the vicinity" of the microdialysis probes filled with a $25 \%$ $\mathrm{CO}_{2}$ microdialysis solution recorded a change equivalent to only 6 to $7 \mathrm{mmHg}$ rise in $\mathrm{PaCO}_{2}$. In a similar set-up in an anesthetized preparation $25 \% \mathrm{CO}_{2}$ caused changes equivalent to 63 Torr end-expiratory $\mathrm{CO}_{2}$ (Li et al., 1999). This indicates the $\mathrm{pH}$ gradient is extremely steep near the dialysis probe. Pharmacogenetic inhibition of RTN neurons has been done using the allato-statin receptor method (Tan et al., 2006; Marina et al., 2010). Allatostatin produced a 60\% reduction in the HCVR but did not affect resting breathing. Although this contradicts my recent findings the differences could be that the allatostatin receptor expressed on less than half of the RTN neurons and on nearby C1 cells. Evidence more recently supports the view that the RTN mediates the effect of $\mathrm{CO}_{2}$ on breathing (both $\mathrm{F}_{\mathrm{R}}$ and $\mathrm{V}_{\mathrm{T}}$ ). Muscimol, a GABA agonist, injected into the area of the RTN resulted in hypopnea (Takakura et al., 2013) and deleting the RTN more specifically through genetics produced hypoventilation in mice (Ramanantsoa et al., 2011).

Utilizing the green light-activated proton pump archaerhodopsinT 3.0 allowed us to instantaneously and reversibly inhibit the RTN population with little off-target effects. The speed and precision of the technique does not allow compensation by other CRC's or peripheral chemoreceptors ( $\mathrm{CBs}$ ) and gives a measure of the instantaneous contribution of the transduced population of neurons to breathing. At rest, the results showed that the Phox $2 \mathrm{~b}$ neuronal population drives ventilation $\left(F_{R}\right.$ and $\left.V_{T}\right)$ during quiet waking/non-REM sleep and only affects $V_{T}$ in REM sleep in unanesthetized rats.

\subsection{Hypoxia Silences the RTN}


Gesell et al. (1940) hypothesized that, "hyperpnea, caused either by oxygen scarcity, or carbon dioxide excess, tends to become exclusively reflexogenic (peripheral) or centrogenic. In other words, one mechanism gains the upper hand over the other and maintains primary control." Our work over the past few years has uncovered the probable neuronal mechanism responsible for these old physiological observations.

It has been shown in the past that RTN neurons are $\mathrm{pH}$ and/or $\mathrm{PaCO}_{2}$ sensitive, with $\mathrm{F}_{\mathrm{R}}$ and $\mathrm{V}_{\mathrm{T}}$ increasing with a lower $\mathrm{pH}$ and higher $\mathrm{PaCO}_{2}$ (Guyenet et al., 2005). Guyenet et al. (2005) and Basting et al. (2015) (see chapter 2) show RTN neurons recorded in vivo (anesthetized rats) are silenced at a pH of 7.53 or higher and $\mathrm{PaCO}_{2}$ below $32 \mathrm{mmHg}$. RTN neurons in slices or isolated from slices have similar characteristics (Lazarenko et al., 2009; Wang et al., 2013c).

The evidence that the putative CRC's (RTN) are inhibited by hypocapnic hypoxia is shown in chapter 2 with archaerhodopsinT 3.0 studies in conscious rats. RTN photoinhibition decreased the breathing rate under hyperoxia and normoxia but the effect disappeared under $12 \% \mathrm{FiO}_{2}$ strongly suggesting that neurons are silent under the latter condition. When acetazolamide, a carbonic anhydrase inhibitor which reacidifies the blood was administered to rats breathing $12 \% \mathrm{O}_{2}$, RTN photoinhibition again reduced breathing, suggesting that hypoxia silences RTN via alkalosis. Finally, when channelrhodopsin2 is expressed in the RTN and the transduced neurons are photoactivated under hypocapnic hypoxia $F_{R}$ and $V_{T}$ increase dramatically (Basting et al., 2015). Data demonstrate that RTN neurons are still capable of increasing breathing under hypoxia when they are activated and reinforces the conclusion that they are silent under hypoxia presumably because of alkalization of their environment.

\subsection{State-dependent Control of Respiration}


Respiratory chemoreflexes operate to some degree in all states of vigilance but different aspects $\left(F_{R}, V_{T}\right)$ are affected in quiet wake/non-REM vs. REM. In quiet wake and non-REM sleep $\mathrm{pH} / \mathrm{PaCO}_{2}$ is maintained at a constant level through a combination of changes in $\mathrm{F}_{\mathrm{R}}$ and $\mathrm{V}_{\mathrm{T}}$. However, during REM sleep the hypercapnic ventilatory reflex is reduced to only changes in $V_{T}$ and no longer alters breathing frequency (Fagenholz et al., 1976; Sullivan et al., 1979; Coote, 1982; Douglas et al., 1982a; Douglas et al., 1982b; Berthon-Jones \& Sullivan, 1984; Smith et al., 1997; Horner et al., 2002). Furthermore, Orem et al. (2005) showed that in hyperventilated conscious cats diaphragmatic EMG recordings re-emerged during REM sleep that were absent during non-REM sleep. This suggests that timing of each breath is imposed by inputs that are external to the pattern generator and are not under the control of the CRCs. The region of the brain that controls the timing of inspirations during REM sleep is unknown.

Once again through the use of photoinhibition of Arch-expressing RTN neurons we were able to quantify the role RTN neurons play in different states of vigilance. Results showed that RTN neuronal activity directly related to the hypercapnic ventilatory reflex. The hypercapnic ventilatory reflex is identical during quiet wake and non-REM sleep; RTN neurons are affecting both the $F_{R}$ and $V_{T}$. During the REM state when RTN neurons are photoinhibited only a change in $V_{T}$ is observed.

Observations during REM sleep show a greatly attenuated chemoreflex with only $\mathrm{V}_{\mathrm{T}}$ shifting with $\mathrm{CO}_{2}$. Other areas of the brain that usually detect $\mathrm{PaCO}_{2}$, such as serotonergic and noradrenergic groups, are silent during REM sleep (Foote et al., 1983; Veasey et al., 1995). During REM sleep there is paralysis of airways and other respiratory muscles (atonia), breathing becomes irregular and frequency increases (Fraigne \& Orem, 2011). This phenomenon suggests 
that the central pattern generator is no longer able to produce a steady unvarying pattern of breathing.

\subsection{RTN's Role after Peripheral Chemoreceptor Denervation}

I found that RTN neurons retain the ability to regulate respiration in a $\mathrm{pH} / \mathrm{PaCO}_{2}$ dependent manner after carotid body denervation (CBD) (Takakura et al., 2006; Basting et al., 2016). Blood gases and ventilation measurements return to baseline one week after CBD confirming previous findings in rats (Mouradian et al., 2012). Shown in this dissertation (Chapter 4) is evidence that RTN inhibition with ArchT 3.0 resulted in greater inhibition of respiration after $\mathrm{CBD}$ than pre-CBD, except during hyperoxic conditions. Initially, these data suggest that there is a certain amount of central plasticity that is compensating for the loss of the peripheral drive to breathe. An examination of the kinetics of the response during the photoinhibition of the RTN neurons, however, provides insight into the dynamics and interplay between the peripheral and central chemoreceptors. The fast response (3-4s) to low $\mathrm{PaO}_{2}$ during photoinhibition induced hypopnea by the CBs no longer remains after CBD (See section 5.5 for interpretation).

Carotid body denervation caused an initial acidic shift in the arterial blood gasses of the body as expected that normalized over time. In the rat, it can take up to 10 weeks for the $\mathrm{PaCO}_{2}$ to recover back to pre-CBD values (Olson et al., 1988b). However, Mouradian et al. show a complete recovery of blood gasses, including $\mathrm{PaCO}_{2}$, in seven days or less (Mouradian et al., 2012; Basting et al., 2016). Also described in work accomplished previously that we confirmed, was the nearly complete absence of the hypoxic ventilatory reflex (HVR) 6-7 days post-CBD (Mouradian et al., 2012). The HVR has been shown to increase overall respiration $~ 50 \%$ in hypoxic conditions $\left(12 \% \mathrm{FiO}_{2}\right.$ ) (Hodges et al., 2002) in healthy rodents. Importantly, despite the 
return of blood gas levels and respiration to baseline levels in normoxic conditions, in hypoxic conditions the peripherally denervated animals' blood gas measurements and HVR were significantly altered. CBD rats' blood gas measurements differed from their pre-CBD values in hypoxic environments, showing changes in $\mathrm{pH}, \mathrm{PaCO}_{2}, \mathrm{PaO}_{2}$, and $\mathrm{HCO}_{3}\left(\downarrow \mathrm{pH}, \uparrow \mathrm{PaCO}_{2}, \downarrow \mathrm{PaO}_{2}\right.$, $\left.\uparrow \mathrm{HCO}_{3}\right)$. These shifts taken together move in a direction that would keep the central chemoreceptors (RTN included) in an activated state (Basting et al., 2016).

After CBD, hypercapnia still remains a powerful stimulus on breathing frequency and the RTN is still sufficient and necessary to drive breathing. Optogenetic stimulation of RTN neurons increases mainly $F_{R}$ in quietly awake and non-REM states in rats and mice including conditions where CBs are silent (hyperoxia) (Abbott et al., 2011; Burke et al., 2015b; Holloway et al., 2015; Kumar et al., 2015). Immediate and reversible inhibition of RTN neurons through optogenetics after CBD results in an immediate decrease in respiration. Results shown previously in chapter 4 demonstrate that after CBD the RTN neurons' drive on breathing is still proportional to the changes in $\mathrm{pH} / \mathrm{PaCO}_{2}$.

\subsection{Is Central Plasticity Responsible for Recovery After CBD?}

Blood gasses return to baseline levels 4-7 days after CBD in rats maintained in a normoxic environment. Despite large fluctuations in blood gas levels directly following CBD and a lack of recovery of the hypoxic ventilatory reflex the rat is able to return blood gasses back to control levels after a week. (Mouradian et al., 2012; Basting et al., 2016). Mouradian et al. observed that after $\mathrm{CBD}$ in three rat strains the hypercapnic ventilatory reflex was normal after two weeks of recovery. They interpreted this finding as a neuromodulated "respiratory plasticity" by the central chemoreceptors (Hodges \& Forster, 2012; Mouradian et al., 2012). However, through analysis of the breathing kinetics during $10 \mathrm{~s}$ inhibition of the RTN neurons in 
different $\mathrm{FiO}_{2}$ conditions we were able to draw alternative conclusions (chapters 2 and 4).

Normally, the CBs provide a tonic input on the drive to breathe but when that input is no longer there (after $\mathrm{CBD}$ ) kinetics of breathing differ under optogenetic inhibition of RTN neurons. The RTN plays a more critical role in the drive to breathe after CBD in conditions that had silenced them previously (e.g. hypocapnic hypoxia) due to a $\mathrm{PaCO}_{2}$ enriched, acidic environment that keeps the RTN neurons firing.

When originally interpreting the results of data collected 7 days after CBD the hypothesis that the RTN neurons are firing more frequently and thus maintaining a greater influence on breathing and compensating for the loss of the peripheral chemoreceptors under normoxic conditions was supported. Hypopnea was greater during the inhibition of RTN neurons after CBD was performed in normoxia indicating a greater role of this CCR. When the breathing response kinetics during optogenetic inhibition was analyzed, however, an alternative conclusion emerged. Although the total inhibition of breathing was greater after CBD, we made two observations of note. First, the initial decrease caused by the inhibition of the RTN neurons on breathing before and after CBD was roughly equivalent. This result indicated that at any moment in normoxia, before or after CBD, RTN neurons provided the same amount of tonic drive to breathe. Secondly, after CBD, the original level of hypopnea evoked by the silencing of RTN neurons remained throughout the full $10 \mathrm{~s}$ stimulus. Previous to CBD, inhibition of the RTN neurons resulted in a relatively brief but severe hypopnea that was followed by a gradual recovery towards the baseline level starting $\sim 3$ seconds into RTN photoinhibition. After CBD, the initial level of hypopnea remained throughout the entirety of the $10 \mathrm{~s}$ stimulus. When the laser stimulus terminated it took the CBD rats a significantly longer time to recover to baseline $V_{E}$ levels. These data suggest, in carotid body intact rats, the fast response ( $3 \mathrm{~s})$ of the CBs to 
hypoxia is responsible for the gradual rise in ventilation during the $10 \mathrm{~s}$ period of RTN photoinhibition. In other words, the greater inhibition observed after CBD is not due to an increased initial contribution of RTN neurons but to the lack of CB input to RTN.

Consistent with this interpretation is the data collected while the rats were exposed to hyperoxia before and after CBD. Hyperoxia silences the peripheral chemoreceptors, reduces breathing and acidifies the arterial blood causing a restoration of breathing via RTN activation. As a result, inhibiting the RTN has the greatest effect on respiration during hyperoxia because the peripheral drive on breathing is no longer present. Interestingly, there is no difference in the amount of inhibition observed before and after CBD when the rats are in $65 \% \mathrm{FiO}_{2}$. When respiratory response kinetics were analyzed under this condition before, during and after photoinhibition (10 s) there were no differences observed once again. There was no recovery from the initial hypopnea during RTN inhibition over the $10 \mathrm{~s}$. After the laser was turned off it took a long time for the rats to recover to baseline breathing levels. Similar to CBD rats in other conditions, under hyperoxia both rats before and after CBD displayed the hypopnea that did not show any type of recovery during photoinhibition of the RTN and took a long time to recover to baseline indicating the absence of the speedy HVR provided by the peripheral chemoreceptors.

\subsection{Limitations}

The optogenetic methodology used throughout this dissertation has the following limitations. Lentiviral vectors with the artificial promoter PRSx8 transduce both RTN and the neighboring C1 neurons (Abbott et al., 2009b). The C1 neurons are catecholaminergic cells that regulate blood pressure primarily but, as shown recently, also activate breathing when they are stimulated (Abbott et al., 2013a). C1 cell inhibition could therefore have contributed to the breathing effects described in this dissertation. Secondly, we were never able to transduce more 
than $50 \%$ of RTN neurons with Arch and therefore we were not been able to observe the full extent of the control of breathing by RTN neurons.

In future experiments, it will be necessary to find a way to transduce RTN neurons with ChR2 or ArchT more selectively, i.e. without transducing the C1 cells. Towards this goal I have tried to use Fenno et al.'s Boolean logic vector approach (Fenno et al., 2014). This vector expresses its payload (e.g. ChR2 or Arch) in the presence of Cre recombinase but the transgene is excised by the FlpO recombinase (Fenno et al., 2014). I have therefore used DBH-FlpO mice that express $\mathrm{FlpO}$ in all catecholamine neurons including the $\mathrm{C} 1$ cells but do not express $\mathrm{FlpO}$ in the RTN. To introduce Cre into both C1 and RTN neurons, I used a lentiviral vector that expresses Cre under the PRSx8 promotor. Then I microinjected Fenno's AAV Cre $\mathrm{ON} / \mathrm{FlpO}_{\mathrm{OFF}}-\mathrm{ChR2}$ (the ArchT inhibitory construct is still being developed) into the RTN. In theory, this approach should have introduced ChR2 into the Cre+ RTN neurons while excluding the opsin from the FlpO+ $\mathrm{C} 1$ neurons. Unfortunately, histological results still showed that ChR2 was expressed in both C1 and RTN neurons. This non-specific expression may be explained in one of two ways. First, the FlpO gene is not expressed by all $\mathrm{C} 1$ cells. This explanation is probably incorrect because FlpO transcripts were detected in essentially every $\mathrm{C} 1$ cell by in situ hybridization. The alternative explanation is that FlpO was not expressed at a sufficiently high level to turn off ChR2 production. By contrast Cre was introduced with a lentiviral vector and therefore was presumably expressed at very high level. A method capable of complete and selective transduction of RTN neurons remains to be developed.

\section{Conclusions}

RTN neurons drive the respiratory system through excitatory connections to the respiratory column and do so by sensing the $\mathrm{PaCO}_{2} / \mathrm{pH}$ of the local environment. Their unique 
location, detection system and projection pattern all indicate that the RTN is the strongest putative central chemoreceptor candidate. Selective stimulation, pharmacologically or optogenetically, in vivo and in vitro lead to increased firing rate and respiratory drive similar to that observed in hypercapnic conditions. Alternatively, inhibition of RTN neurons has the opposite effect. When RTN populations were inhibited baseline breathing decreased in normoxia and there is greater reduction in ventilation in hypercapnic conditions.

Under hypocapnic hypoxic conditions the peripheral chemoreceptors (e.g. carotid bodies) provide the excitatory input to the respiratory column through a pathway that circumvents the RTN resulting in an increase in respiration. Due to the increased respiration during hypoxia the $\mathrm{PaCO}_{2}$ decreases and the $\mathrm{pH}$ increases to levels that silence the RTN and eliminate their influence on breathing. One week after the CB's were denervated, removing the largest part of the peripheral chemoreflex, baseline respiration did not increase to pre-CBD levels under hypoxic conditions and the arterial blood pH did not alkalize to the same degree. Breathing inhibition remained after CBD under hypoxic conditions indicating the RTN neurons were providing a drive to breathe in conditions that silenced them previously.

At present, we do not know whether the RTN is the only group of central respiratory chemoreceptors. RTN might be essential to $\mathrm{CO}_{2}$ homeostasis during low metabolic states like non-REM sleep but less so under other conditions (e.g. exercise or grooming). Existing technology (ChR2, Arch, allatostatin) does not transduce even $50 \%$ of the RTN neurons with Arch reaching $<35 \%$ of the RTN population. However, this amount of expression is enough to mediate at least $60 \%$ of the HCVR. Furthermore, the vector-based approach gets around many of the limitations that are present in lesion and pharmacological (non-specific effects) and genetic animal model (compensation over time) studies. An approach that combines specificity 
and greater transduction efficiency that keeps the system intact and healthy is necessary to continue to learn more about the RTN. 


\section{References}

Abbott SB, Coates MB, Stornetta RL \& Guyenet PG. (2013a). Optogenetic Stimulation of C1 and Retrotrapezoid Nucleus Neurons Causes Sleep State-Dependent Cardiorespiratory Stimulation and Arousal in Rats. Hypertension 61, 835-841.

Abbott SB, Depuy SD, Nguyen T, Coates MB, Stornetta RL \& Guyenet PG. (2013b). Selective optogenetic activation of rostral ventrolateral medullary catecholaminergic neurons produces cardiorespiratory stimulation in conscious mice. J Neurosci 33, 3164-3177.

Abbott SB, DePuy SD, Nguyen T, Coates MB, Stornetta RL \& Guyenet PG. (2013c). Selective optogenetic activation of rostral ventrolateral medullary catecholaminergic neurons produces cardiorespiratory stimulation in conscious mice. J Neurosci 33, 3164-3177.

Abbott SB, Holloway BB, Viar KE \& Guyenet PG. (2014). Vesicular glutamate transporter 2 is required for the respiratory and parasympathetic activation produced by optogenetic stimulation of catecholaminergic neurons in the rostral ventrolateral medulla of mice in vivo. Eur J Neurosci 39, 98-106.

Abbott SB, Stornetta RL, Coates MB \& Guyenet PG. (2011). Phox2b-expressing neurons of the parafacial region regulate breathing rate, inspiration, and expiration in conscious rats. $J$ Neurosci 31, 16410-16422.

Abbott SB, Stornetta RL, Fortuna MG, Depuy SD, West GH, Harris TE \& Guyenet PG. (2009a). Photostimulation of retrotrapezoid nucleus phox $2 \mathrm{~b}$-expressing neurons in vivo produces long-lasting activation of breathing in rats. J Neurosci 29, 5806-5819.

Abbott SB, Stornetta RL, Socolovsky CS, West GH \& Guyenet PG. (2009b). Photostimulation of channelrhodopsin-2 expressing ventrolateral medullary neurons increases sympathetic nerve activity and blood pressure in rats. J Physiol 587, 5613-5631.

Ainslie PN \& Duffin J. (2009). Integration of cerebrovascular CO2 reactivity and chemoreflex control of breathing: mechanisms of regulation, measurement, and interpretation. Am J Physiol Regul Integr Comp Physiol 296, R1473-R1495.

Amiel J, Laudier B, Attie-Bitach T, Trang H, de PL, Gener B, Trochet D, Etchevers H, Ray P, Simonneau M, Vekemans M, Munnich A, Gaultier C \& Lyonnet S. (2003). Polyalanine expansion and frameshift mutations of the paired-like homeobox gene PHOX2B in congenital central hypoventilation syndrome. Nat Genet 33, 459-461. 
Angelova PR, Kasymov V, Christie I, Sheikhbahaei S, Turovsky E, Marina N, Korsak A, Zwicker J, Teschemacher AG, Ackland GL, Funk GD, Kasparov S, Abramov AY \& Gourine AV. (2015). Functional Oxygen Sensitivity of Astrocytes. J Neurosci 35, 10460-10473.

Ballantyne D \& Richter DW. (1986). The non-uniform character of expiratory synaptic activity in expiratory bulbospinal neurones of the cat. Journal of Physiology 370, 433-456.

Barnett S, Mulligan E, Wagerle LC \& Lahiri S. (1988). Measurement of carotid body blood flow in cats by use of radioactive microspheres. J Appl Physiol (1985) 65, 2484-2489.

Bartlett D, Jr. (1971). Origin and regulation of spontaneous deep breaths. RespirPhysiol 12, 230238.

Basting TM, Abe C, Viar KE, Stornetta RL \& Guyenet PG. (2016). Is plasticity within the retrotrapezoid nucleus responsible for the recovery of the PCO set-point after carotid body denervation in rats? The Journal of physiology.

Basting TM, Burke PG, Kanbar R, Viar KE, Stornetta DS, Stornetta RL \& Guyenet PG. (2015). Hypoxia silences retrotrapezoid nucleus respiratory chemoreceptors via alkalosis. $J$ Neurosci 35, 527-543.

Bautista TG, Burke PG, Sun QJ, Berkowitz RG \& Pilowsky PM. (2010). The generation of postinspiratory activity in laryngeal motoneurons: a review. Advances in experimental medicine and biology 669, 143-149.

Bell HJ, Ferguson C, Kehoe V \& Haouzi P. (2009). Hypocapnia increases the prevalence of hypoxia-induced augmented breaths. AmJPhysiol RegullntegrComp Physiol 296, R334R344.

Bell HJ \& Haouzi P. (2010). The hypoxia-induced facilitation of augmented breaths is suppressed by the common effect of carbonic anhydrase inhibition. Respiratory physiology \& neurobiology 171, 201-211.

Berssenbrugge A, Dempsey J, Iber C, Skatrud J \& Wilson P. (1983). Mechanisms of hypoxiainduced periodic breathing during sleep in humans. The Journal of physiology $343,507-$ 524. 
Berthon-Jones M \& Sullivan CE. (1984). Ventilation and arousal responses to hypercapnia in normal sleeping humans. J Appl Physiol 57, 59-67.

Bianchi AL, Denavit-Saubie M \& Champagnat J. (1995). Central control of breathing in mammals: neuronal circuitry, membrane properties, and neurotransmitters. PhysiolRev 75, 1-45.

Blain GM, Smith CA, Henderson KS \& Dempsey JA. (2009). Contribution of the carotid body chemoreceptors to eupneic ventilation in the intact, unanesthetized dog. J Appl Physiol 106, 1564-1573.

Blain GM, Smith CA, Henderson KS \& Dempsey JA. (2010). Peripheral chemoreceptors determine the respiratory sensitivity of central chemoreceptors to CO(2). J Physio/ 588, 2455-2471.

Bochorishvili G, Stornetta RL, Coates MB \& Guyenet PG. (2012). Pre-Botzinger complex receives glutamatergic innervation from galaninergic and other retrotrapezoid nucleus neurons. $J$ Comp Neurol 520, 1047-1061.

Brown DL \& Guyenet PG. (1985). Electrophysiological study of cardiovascular neurons in the rostral ventrolateral medulla in rats. Circ Res 56, 359-369.

Burgess KR. (1997). Central sleep apnoea and heart failure (Part I). Respirology 2, 243-253.

Burke PG, Abbott SB, Coates MB, Viar KE, Stornetta RL \& Guyenet PG. (2014). Optogenetic stimulation of adrenergic $C 1$ neurons causes sleep state-dependent cardiorespiratory stimulation and arousal with sighs in rats. Am J Respir Crit Care Med 190, 1301-1310.

Burke PG, Kanbar R, Basting TM, Hodges WM, Viar KE, Stornetta RL \& Guyenet PG. (2015a). State-dependent control of breathing by the retrotrapezoid nucleus. J Physio/ 593, 29092926.

Burke PG, Kanbar R, Basting TM, Hodges WM, Viar KE, Stornetta RL \& Guyenet PG. (2015b). State-dependent control of breathing by the retrotrapezoid nucleus. J Physiol 593, 2909-2926.

Burke PG, Li Q, Costin ML, McMullan S, Pilowsky PM \& Goodchild AK. (2008). Somatostatin 2A receptor-expressing presympathetic neurons in the rostral ventrolateral medulla maintain blood pressure. Hypertension 52, 1127-1133. 
Carroll MS, Patwari PP, Kenny AS, Brogadir CD, Stewart TM \& Weese-Mayer DE. (2014). Residual chemosensitivity to ventilatory challenges in genotyped congenital central hypoventilation syndrome. J Appl Physiol (1985) 116, 439-450.

Chang AJ, Ortega FE, Riegler J, Madison DV \& Krasnow MA. (2015). Oxygen regulation of breathing through an olfactory receptor activated by lactate. Nature 527, 240-244.

Chow BY, Han X, Dobry AS, Qian X, Chuong AS, Li M, Henninger MA, Belfort GM, Lin Y, Monahan PE \& Boyden ES. (2010). High-performance genetically targetable optical neural silencing by light-driven proton pumps. Nature 463, 98-102.

Coles SK, Miller R, Huela J, Wolken P \& Schlenker E. (2002). Frequency responses to hypoxia and hypercapnia in carotid body-denervated conscious rats. Respiratory physiology \& neurobiology 130, 113-120.

Coote JH. (1982). Respiratory and circulatory control during sleep. Journal of Experimental Biology 100, 223-244.

Curran AK, Rodman JR, Eastwood PR, Henderson KS, Dempsey JA \& Smith CA. (2000). Ventilatory responses to specific CNS hypoxia in sleeping dogs. J Appl Physiol 88, 1840-1852.

Dauger S, Pattyn A, Lofaso F, Gaultier C, Goridis C, Gallego J \& Brunet JF. (2003). Phox2b controls the development of peripheral chemoreceptors and afferent visceral pathways. Development 130, 6635-6642.

De Castro F. (1926). Sur la structure et l'innervation de la glande intercarotidienne (glomus caroticum) de l'homme et des mammiferes et sur un nouveau systeme de l'innervation autonome du nerf glossopharyngien. Trav Lab Rech Biol 24, 365-432.

de Castro F. (2009). Towards the sensory nature of the carotid body: hering, de castro and heymansdagger. Front Neuroanat 3, 23.

Dejours P. (1962). Chemoreflexes in breathing. Physiol Rev 42, 335-358.

Del Rio R, Marcus NJ \& Schultz HD. (2013). Carotid chemoreceptor ablation improves survival in heart failure: rescuing autonomic control of cardiorespiratory function. J Am Coll Cardiol 62, 2422-2430. 
Dempsey JA, Smith CA, Blain GM, Xie A, Gong Y \& Teodorescu M. (2012). Role of central/peripheral chemoreceptors and their interdependence in the pathophysiology of sleep apnea. Adv Exp Med Biol 758, 343-349.

Dobbins EG \& Feldman JL. (1994). Brainstem network controlling descending drive to phrenic motoneurons in rat. J Comp Neurol 347, 64-86.

Doi A \& Ramirez JM. (2008). Neuromodulation and the orchestration of the respiratory rhythm. Respir Physiol Neurobiol 164, 96-104.

Doi A \& Ramirez JM. (2010). State-dependent interactions between excitatory neuromodulators in the neuronal control of breathing. J Neurosci 30, 8251-8262.

Douglas NJ, White DP, Weil JV, Pickett CK, Martin RJ, Hudgel DW \& Zwillich CW. (1982a). Hypoxic ventilatory response decreases during sleep in normal men. Am Rev Respir Dis 125, $286-$ 289.

Douglas NJ, White DP, Weil JV, Pickett CK \& Zwillich CW. (1982b). Hypercapnic ventilatory response in sleeping adults. Am Rev Respir Dis 126, 758-762.

Dubreuil V, Ramanantsoa N, Trochet D, Vaubourg V, Amiel J, Gallego J, Brunet JF \& Goridis C. (2008). A human mutation in Phox $2 \mathrm{~b}$ causes lack of CO2 chemosensitivity, fatal central apnoea and specific loss of parafacial neurons. Proc Natl Acad Sci USA 105, 1067-1072.

Duchen MR, Caddy KW, Kirby GC, Patterson DL, Ponte J \& Biscoe TJ. (1988). Biophysical studies of the cellular elements of the rabbit carotid body. Neuroscience 26, 291-311.

Dutschmann M \& Herbert H. (2006). The Kolliker-Fuse nucleus gates the postinspiratory phase of the respiratory cycle to control inspiratory off-switch and upper airway resistance in rat. Eur J Neurosci 24, 1071-1084.

Erlichman JS, Leiter JC \& Gourine AV. (2010). ATP, glia and central respiratory control. Respir Physiol Neurobiol 173, 305-311.

Fagenholz SA, O'Connell K \& Shannon DC. (1976). Chemoreceptor function and sleep state in apnea. Pediatrics 58, 31-36. 
Farney RJ, Walker JM, Cloward TV \& Rhondeau S. (2003). Sleep-disordered breathing associated with long-term opioid therapy. Chest 123, 632-639.

Fatemian M, Nieuwenhuijs DJ, Teppema LJ, Meinesz S, van der Mey AG, Dahan A \& Robbins PA. (2003). The respiratory response to carbon dioxide in humans with unilateral and bilateral resections of the carotid bodies. The Journal of physiology 549, 965-973.

Feldman JL, Del Negro CA \& Gray PA. (2013). Understanding the rhythm of breathing: so near, yet so far. Annu Rev Physiol 75, 423-452.

Fenno LE, Mattis J, Ramakrishnan C, Hyun M, Lee SY, He M, Tucciarone J, Selimbeyoglu A, Berndt A, Grosenick L, Zalocusky KA, Bernstein H, Swanson H, Perry C, Diester I, Boyce FM, Bass CE, Neve R, Huang ZJ \& Deisseroth K. (2014). Targeting cells with single vectors using multiple-feature Boolean logic. Nat Methods 11, 763-772.

Fiamma MN, O'Connor ET, Roy A, Zuna I \& Wilson RJ. (2013). The essential role of peripheral respiratory chemoreceptor inputs in maintaining breathing revealed when $\mathrm{CO} 2$ stimulation of central chemoreceptors is diminished. JPhysiol 591, 1507-1521.

Finley JCW \& Katz DM. (1992). The central organization of carotid body afferent projections to the brainstem of the rat. Brain Res 571(2), 108-116.

Fleming PJ, Cade D, Bryan MH \& Bryan AC. (1980). Congenital central hypoventilation and sleep state. Pediatrics 66, 425-428.

Foote SL, Bloom FE \& Aston-Jones G. (1983). Nucleus locus coeruleus: New evidence of anatomical and physiological specificity. Physiological Reviews 63, 844-914.

Forster HV. (2003). Plasticity in the control of breathing following sensory denervation. J Appl Physiol 94, 784-794.

Forster HV \& Smith CA. (2010). Contributions of central and peripheral chemoreceptors to the ventilatory response to CO2/H+. JApp/Physiol 108, 989-994.

Fraigne JJ \& Orem JM. (2011). Phasic motor activity of respiratory and non-respiratory muscles in REM sleep. Sleep 34, 425-434. 
Gesell R, Lapides J \& Levin M. (1940). The interaction of central and peripheral control of breathing. Am J Physiol 130, 155-170.

Gestreau C, Heitzmann D, Thomas J, Dubreuil V, Bandulik S, Reichold M, Bendahhou S, Pierson P, Sterner C, Peyronnet-Roux J, Benfriha C, Tegtmeier I, Ehnes H, Georgieff M, Lesage F, Brunet JF, Goridis C, Warth R \& Barhanin J. (2010). Task2 potassium channels set central respiratory CO2 and $\mathrm{O} 2$ sensitivity. Proc Natl Acad Sci USA 107, 2325-2330.

Gonzalez C, Almaraz L, Obeso A \& Rigual R. (1994). Carotid body chemoreceptors: from natural stimuli to sensory discharges. Physiol Rev 74, 829-898.

Gourine AV \& Kasparov S. (2011). Astrocytes as brain interoceptors. Exp Physiol 96, 411-416.

Gourine AV, Kasymov V, Marina N, Tang F, Figueiredo MF, Lane S, Teschemacher AG, Spyer KM, Deisseroth K \& Kasparov S. (2010). Astrocytes control breathing through pH-dependent release of ATP. Science 329, 571-575.

Gourine AV, Wood JD \& Burnstock G. (2009). Purinergic signalling in autonomic control. Trends Neurosci 32, 241-248.

Gray PA, Rekling JC, Bocchiaro CM \& Feldman JL. (1999). Modulation of respiratory frequency by peptidergic input to rhythmogenic neurons in the PreBotzinger complex. Science 286, 1566-1568.

Guyenet PG. (2014a). Regulation of breathing and autonomic outflows by chemoreceptors. Compr Physiol 4, 1511-1562.

Guyenet PG. (2014b). Regulation of breathing and autonomic outflows by chemoreceptors. Compr Physiol 4, 1511-1562.

Guyenet PG, Abbott SB \& Stornetta RL. (2012). The respiratory chemoreception conundrum: Light at the end of the tunnel? Brain Res.

Guyenet PG, Abbott SB \& Stornetta RL. (2013a). The respiratory chemoreception conundrum: light at the end of the tunnel? Brain Res 1511, 126-137.

Guyenet PG \& Bayliss DA. (2015). Neural Control of Breathing and CO2 Homeostasis. Neuron 87, 946-961. 
Guyenet PG, Bayliss DA, Stornetta RL, Ludwig MG, Kumar NN, Shi Y, Burke PG, Kanbar R, Basting TM, Holloway BB \& Wenker IC. (2016). Proton detection and breathing regulation by the retrotrapezoid nucleus. J Physiol 594, 1529-1551.

Guyenet PG \& Mulkey DK. (2010). Retrotrapezoid nucleus and parafacial respiratory group. RespirPhysiol Neurobiol 173, 244-255.

Guyenet PG, Mulkey DK, Stornetta RL \& Bayliss DA. (2005). Regulation of ventral surface chemoreceptors by the central respiratory pattern generator. J Neurosci 25, 8938-8947.

Guyenet PG, Stornetta RL \& Bayliss DA. (2010). Central respiratory chemoreception. J Comp Neurol 518, 3883-3906.

Guyenet PG, Stornetta RL, Bochorishvili G, Depuy SD, Burke PG \& Abbott SB. (2013b). C1 neurons: the body's EMTs. Am J Physiol Regul Integr Comp Physiol 305, R187-204.

Guz A. (1997). Brain, breathing and breathlessness. Respir Physiol 109, 197-204.

Han X, Chow BY, Zhou H, Klapoetke NC, Chuong A, Rajimehr R, Yang A, Baratta MV, Winkle J, Desimone R \& Boyden ES. (2011). A high-light sensitivity optical neural silencer: development and application to optogenetic control of non-human primate cortex. Front Syst Neurosci 5, 18.

Haouzi P \& Bell HJ. (2009). Control of breathing and volitional respiratory rhythm in humans. Journal of applied physiology 106, 904-910.

Haxhiu MA, van Lunteren E, Mitra J \& Cherniack NS. (1987). Comparison of the response of diaphragm and upper airway dilating muscle activity in sleeping cats. Resp Physiol 70, 183-193.

Hayasaka K SA. (2014). Congenital central hypoventilation syndrome: paradigm shifts and future prospects. Nihon Rinsho (Japanese Clinical 72.

Herndon RM \& Coyle JT. (1977). Selective destruction of neurons by a transmitter agonist. Science 198, 71-72. 
Heymans C \& Bouckaert JJ. (1930). Sinus caroticus and respiratory reflexes: I. Cerebral blood flow and respiration. Adrenaline apnoea. The Journal of physiology 69, 254-266.

Hodges MR \& Forster HV. (2012). Respiratory neuroplasticity following carotid body denervation: Central and peripheral adaptations. Neural Regen Res 7, 1073-1079.

Hodges MR, Forster HV, Papanek PE, Dwinell MR \& Hogan GE. (2002). Ventilatory phenotypes among four strains of adult rats. JApplPhysiol 93, 974-983.

Hodges MR, Opansky C, Qian B, Davis S, Bonis JM, Krause K, Pan LG \& Forster HV. (2005). Carotid body denervation alters ventilatory responses to ibotenic acid injections or focal acidosis in the medullary raphe. JAppIPhysiol 98, 1234-1242.

Hodges RD, King AS, King DZ \& French El. (1975). The general ultrastructure of the carotid body of the domestic fowl. Cell Tissue Res 162, 483-497.

Holloway BB, Viar KE, Stornetta RL \& Guyenet PG. (2015). The retrotrapezoid nucleus stimulates breathing by releasing glutamate in adult conscious mice. Eur J Neurosci 42, 2271-2282.

Horner RL. (2009). Emerging principles and neural substrates underlying tonic sleep-statedependent influences on respiratory motor activity. Philos Trans R Soc Lond B Biol Sci 364, 2553-2564.

Horner RL, Liu X, Gill H, Nolan P, Liu H \& Sood S. (2002). Effects of sleep-wake state on the genioglossus vs.diaphragm muscle response to $\mathrm{CO}(2)$ in rats. J Appl Physiol 92, 878-887.

Huckstepp RT \& Dale N. (2011). Redefining the components of central CO2 chemosensitivity-towards a better understanding of mechanism. J Physiol 589, 5561-5579.

Huckstepp RT, Id BR, Eason R, Spyer KM, Dicke N, Willecke K, Marina N, Gourine AV \& Dale N. (2010). Connexin hemichannel-mediated CO2-dependent release of ATP in the medulla oblongata contributes to central respiratory chemosensitivity. J Physiol 588, 3901-3920.

Hwang DY, Carlezon WA, Jr., Isacson O \& Kim KS. (2001). A high-efficiency synthetic promoter that drives transgene expression selectively in noradrenergic neurons. Human Gene Therapy 12, 1731-1740. 
Janczewski WA \& Feldman JL. (2006). Distinct rhythm generators for inspiration and expiration in the juvenile rat. J Physiol 570, 407-420.

Kanbar R, Stornetta RL, Cash DR, Lewis SJ \& Guyenet PG. (2010). Photostimulation of Phox2b medullary neurons activates cardiorespiratory function in conscious rats. Am J Respir Crit Care Med 182, 1184-1194.

Kasymov V, Larina O, Castaldo C, Marina N, Patrushev M, Kasparov S \& Gourine AV. (2013). Differential sensitivity of brainstem versus cortical astrocytes to changes in $\mathrm{pH}$ reveals functional regional specialization of astroglia. J Neurosci 33, 435-441.

Konig SA \& Seller H. (1991). Historical development of current concepts on central chemosensitivity. ArchltalBiol 129, 223-237.

Kumar NN, Velic A, Soliz J, Shi Y, Li K, Wang S, Weaver JL, Sen J, Abbott SB, Lazarenko RM, Ludwig MG, Perez-Reyes E, Mohebbi N, Bettoni C, Gassmann M, Suply T, Seuwen K, Guyenet PG, Wagner CA \& Bayliss DA. (2015). Regulation of breathing by CO2 requires the proton-activated receptor GPR4 in retrotrapezoid nucleus neurons. Science $\mathbf{3 4 8}$, $1255-1260$.

Kumar P \& Prabhakar NR. (2012). Peripheral chemoreceptors: function and plasticity of the carotid body. Comprehensive Physiology 2, 141-219.

Lazarenko RM, Milner TA, Depuy SD, Stornetta RL, West GH, Kievits JA, Bayliss DA \& Guyenet PG. (2009). Acid sensitivity and ultrastructure of the retrotrapezoid nucleus in Phox2b-EGFP transgenic mice. J Comp Neurol 517, 69-86.

Li A \& Nattie E. (2002). CO2 dialysis in one chemoreceptor site, the RTN: stimulus intensity and sensitivity in the awake rat. Respir Physiol Neurobiol 133, 11-22.

Li A \& Nattie EE. (1997). Focal central chemoreceptor sensitivity in the RTN studied with a CO2 diffusion pipette in vivo. JApp/Physiol 83, 420-428.

Li A, Randall M \& Nattie EE. (1999). CO(2) microdialysis in retrotrapezoid nucleus of the rat increases breathing in wakefulness but not in sleep. J Appl Physiol 87, 910-919. 
Lieske SP, Thoby-Brisson M, Telgkamp P \& Ramirez JM. (2000). reconfiguration of the neural network controlling multiple breathing patterns: eupnea, sighs and gasps. NatNeurosci 3, 600-607.

Liu JP, Nakakura T, Tomura H, Tobo M, Mogi C, Wang JQ, He XD, Takano M, Damirin A, Komachi M, Sato K \& Okajima F. (2010). Each one of certain histidine residues in G-proteincoupled receptor GPR4 is critical for extracellular proton-induced stimulation of multiple G-protein-signaling pathways. PharmacolRes 61, 499-505.

Loeschcke HH, Koepchen HP \& GERTZ KH. (1958). Effect of hydrogen ion concentration and carbon dioxide pressure in the cerebrospinal fluid on respiration. Pflugers Arch 266, 569585.

Lonergan T, Teschemacher AG, Hwang DY, Kim KS, Pickering AE \& Kasparov S. (2005). Targeting brain stem centers of cardiovascular control using adenoviral vectors: impact of promoters on transgene expression. Physio/Genomics 20, 165-172.

Lovering AT, Dunin-Barkowski WL, Vidruk EH \& Orem JM. (2003). Ventilatory response of the cat to hypoxia in sleep and wakefulness. Journal of applied physiology 95, 545-554.

Lovering AT, Fraigne JJ, Dunin-Barkowski WL, Vidruk EH \& Orem JM. (2006). Medullary respiratory neural activity during hypoxia in NREM and REM sleep in the cat. $J$ Neurophysiol 95, 803-810.

Lovering AT, Fraigne JJ, Dunin-Barkowski WL, Vidruk EH \& Orem JM. (2012). Tonic and phasic drive to medullary respiratory neurons during periodic breathing. Respiratory physiology \& neurobiology 181, 286-301.

Ludwig MG, Vanek M, Guerini D, Gasser JA, Jones CE, Junker U, Hofstetter H, Wolf RM \& Seuwen K. (2003). Proton-sensing G-protein-coupled receptors. Nature 425, 93-98.

Marcus NJ, R. DR, Schultz EP, Xia XH \& Schultz HD. (2014). Carotid body denervation improves autonomic and cardiac function and attenuates disordered breathing in congestive heart failure. The Journal of physiology 592, 391-408.

Marina N, Abdala AP, Korsak A, Simms AE, Allen AM, Paton JF \& Gourine AV. (2011). Control of sympathetic vasomotor tone by catecholaminergic $\mathrm{C} 1$ neurones of the rostral ventrolateral medulla oblongata. Cardiovasc Res 91, 703-710. 
Marina N, Abdala AP, Trapp S, Li A, Nattie EE, Hewinson J, Smith JC, Paton JF \& Gourine AV. (2010). Essential role of Phox $2 \mathrm{~b}$-expressing ventrolateral brainstem neurons in the chemosensory control of inspiration and expiration. J Neurosci 30, 12466-12473.

Martin-Body RL, Robson GJ \& Sinclair JD. (1986). Restoration of hypoxic respiratory responses in the awake rat after carotid body denervation by sinus nerve section. $J$ Physiol 380, 6174.

Massari VJ, Shirahata M, Johnson TA \& Gatti PJ. (1996). Carotid sinus nerve terminals which are tyrosine hydroxylase immunoreactive are found in the commissural nucleus of the tractus solitarius. J Neurocytol 25, 197-208.

Mattis J, Tye KM, Ferenczi EA, Ramakrishnan C, O'Shea DJ, Prakash R, Gunaydin LA, Hyun M, Fenno LE, Gradinaru V, Yizhar O \& Deisseroth K. (2012). Principles for applying optogenetic tools derived from direct comparative analysis of microbial opsins. Nat Methods 9, 159-172.

McBryde FD, Abdala AP, Hendy EB, Pijacka W, Marvar P, Moraes DJ, Sobotka PA \& Paton JF. (2013). The carotid body as a putative therapeutic target for the treatment of neurogenic hypertension. Nat Commun 4, 2395.

McDonald DM \& Blewett RW. (1981). Location and size of carotid body-like organs (paraganglia) revealed in rats by the permeability of blood vessels to Evans blue dye. J Neurocytol 10, 607-643.

McKay LC \& Feldman JL. (2008). Unilateral ablation of pre-Botzinger complex disrupts breathing during sleep but not wakefulness. Am J Respir Crit Care Med 178, 89-95.

Miller JR, Neumueller S, Muere C, Olesiak S, Pan L, Hodges MR \& Forster HV. (2013). Changes in neurochemicals within the ventrolateral medullary respiratory column in awake goats after carotid body denervation. J Appl Physiol 115, 1088-1098.

Mitchell RA, Loeschcke HH, Massion WH \& Severinghaus JW. (1963a). Respiratory responses mediated through superficial chemosensitive areas on the medulla. J Appl Physiol 18, 523-533. 
Mitchell RA, Loeschcke HH, Severinghaus JW, Richardson JW \& Massion WH. (1963b). Regions of respiratory chemosensitivity on the surface of the medulla. Ann NY Acad Sci 109, 661681.

Mouradian GC, Forster HV \& Hodges MR. (2012). Acute and chronic effects of carotid body denervation on ventilation and chemoreflexes in three rat strains. $J$ Physiol 590, 33353347.

Mukohata Y, Ihara K, Tamura T \& Sugiyama Y. (1999). Halobacterial rhodopsins. J Biochem 125, 649-657.

Mulkey DK, Rosin DL, West G, Takakura AC, Moreira TS, Bayliss DA \& Guyenet PG. (2007). Serotonergic neurons activate chemosensitive retrotrapezoid nucleus neurons by a $\mathrm{pH}-$ independent mechanism. J Neurosci 27, 14128-14138.

Mulkey DK, Stornetta RL, Weston MC, Simmons JR, Parker A, Bayliss DA \& Guyenet PG. (2004). Respiratory control by ventral surface chemoreceptor neurons in rats. Nat Neurosci 7, 1360-1369.

Nakamura A, Zhang W, Yanagisawa M, Fukuda Y \& Kuwaki T. (2007). Vigilance state-dependent attenuation of hypercapnic chemoreflex and exaggerated sleep apnea in orexin knockout mice. J Appl Physiol 102, 241-248.

Nattie E, Shi J \& Li A. (2001). Bicuculline dialysis in the retrotrapezoid nucleus (RTN) region stimulates breathing in the awake rat. RespPhysiol 124, 179-193.

Nattie EE \& Li A. (2002). Substance P-saporin lesion of neurons with NK1 receptors in one chemoreceptor site in rats decreases ventilation and chemosensitivity. J Physiol 544, 603-616.

Nattie EE, Li A \& St John WM. (1991). Lesions in retrotrapezoid nucleus decrease ventilatory output in anesthetized or decerebrate cats. J Appl Physiol 71(4), 1364-1375.

Nattie EE \& Li AH. (1990). Fluorescence location of RVLM kainate microinjections that alter the control of breathing. JApplPhysiol 68, 1157-1166.

Nattie EE, Li, A. (2012). Central Chemoreceptors: Locations and Functions. Comp Physiol 2, 221254. 
Nurse CA. (2014). Synaptic and paracrine mechanisms at carotid body arterial chemoreceptors. The Journal of physiology 592, 3419-3426.

Ohashi S, Izumizaki M, Atsumi T \& Homma I. (2013). CO2 homeostasis is maintained in conscious humans by regulation of tidal volume, but not of respiratory rhythm. Resp Physiol Neurobiol 186, 155-163.

Olson EB, Jr., Vidruk EH \& Dempsey JA. (1988a). Carotid body excision significantly changes ventilatory control in awake rats. Journal of applied physiology 64, 666-671.

Olson EBJ, Vidruk EH \& Dempsey JA. (1988b). Carotid body excision significantly changes ventilatory control in awake rats. JAppIPhysiol 64(2), 666-671.

Onimaru H \& Homma I. (2003). A novel functional neuron group for respiratory rhythm generation in the ventral medulla. J Neurosci 23, 1478-1486.

Orem JM, Lovering AT \& Vidruk EH. (2005). Excitation of medullary respiratory neurons in REM sleep. Sleep 28, 801-807.

Pagliardini S, Janczewski WA, Tan W, Dickson CT, Deisseroth K \& Feldman JL. (2011). Active expiration induced by excitation of ventral medulla in adult anesthetized rats. J Neurosci 31, 2895-2905.

Pan LG, Forster HV, Martino P, Strecker PJ, Beales J, Serra A, Lowry TF, Forster MM \& Forster AL. (1998). Important role of carotid afferents in control of breathing. JApp/Physiol 85, 1299-1306.

Pan LG, Forster HV, Ohtake PJ, Lowry TF, Korducki MJ \& Forster AL. (1995). Effect of carotid chemoreceptor denervation on breathing during ventrolateral medullary cooling in goats. JApplPhysiol 79, 1120-1128.

Pardal R, Ortega-Saenz P, Duran R \& Lopez-Barneo J. (2007). Glia-like stem cells sustain physiologic neurogenesis in the adult mammalian carotid body. Cell 131, 364-377.

Paton JF, Dickinson CJ \& Mitchell G. (2009). Harvey Cushing and the regulation of blood pressure in giraffe, rat and man: introducing 'Cushing's mechanism'. ExpPhysiol 94, 11-17. 
Paxinos G \& Watson C. (1998). The Rat Brain in Stereotaxic Coordinates. Academic Press, San Diego.

Paxinos G \& Watson C. (2005). The Rat Brain in Stereotaxic Coordinates. Elsevier Academic Press, San Diego.

Pinault D. (1996). A novel single-cell staining procedure performed in vivo under electrophysiological control: morpho-functional features of juxtacellularly labeled thalamic cells and other central neurons with biocytin or Neurobiotin. Journal of Neuroscience Methods 65, 113-136.

Potts JT, Rybak IA \& Paton JF. (2005). Respiratory rhythm entrainment by somatic afferent stimulation. J Neurosci 25, 1965-1978.

Prabhakar NR \& Semenza GL. (2015). Oxygen Sensing and Homeostasis. Physiology 30, 340-348.

Ramanantsoa N, Hirsch MR, Thoby-Brisson M, Dubreuil V, Bouvier J, Ruffault PL, Matrot B, Fortin G, Brunet JF, Gallego J \& Goridis C. (2011). Breathing without CO2 chemosensitivity in conditional Phox2b mutants. J Neurosci 31, 12880-12888.

Ramirez JM. (2014). The integrative role of the sigh in psychology, physiology, pathology, and neurobiology. Progress in brain research 209, 91-129.

Ramirez JM, Doi A, Garcia AJ, III, Elsen FP, Koch H \& Wei AD. (2012). The cellular building blocks of breathing. Compr Physiol 2, 2683-2731.

Reis DJ, Golanov EV, Galea E \& Feinstein DL. (1997). Central neurogenic neuroprotection: Central neural systems that protect the brain from hypoxia and ischemia. AnnNY AcadSci 835, 168-186.

Reis DJ, Ruggiero DA \& Morrison SF. (1989). The C1 area of the rostral ventrolateral medulla oblongata. A critical brainstem region for control of resting and reflex integration of arterial pressure. AmJHypertens 2, 363S-374S.

Reyes R, Duprat F, Lesage F, Fink M, Salinas M, Farman N \& Lazdunski M. (1998). Cloning and expression of a novel $\mathrm{pH}$-sensitive two pore domain $\mathrm{K}+$ channel from human kidney. $J$ Biol Chem 273, 30863-30869. 
Richter DW \& Smith JC. (2014). Respiratory rhythm generation in vivo. Physiology 29, 58-71.

Ross LL. (1959). Electron microscopic observations of the carotid body of the cat. J Biophys Biochem Cytol 6, 253-262.

Roux JC, Peyronnet J, Pascual O, Dalmaz Y \& Pequignot JM. (2000). Ventilatory and central neurochemical reorganisation of $\mathrm{O} 2$ chemoreflex after carotid sinus nerve transection in rat. The Journal of physiology 522 Pt 3, 493-501.

Ruffault PL, D'Autreaux F, Hayes JA, Nomaksteinsky M, Autran S, Fujiyama T, Hoshino M, Hagglund M, Kiehn O, Brunet JF, Fortin G \& Goridis C. (2015). The retrotrapezoid nucleus neurons expressing Phox $2 \mathrm{~b}$ and Atoh-1 are essential for the respiratory response to CO2. elife 4, doi: 10.7554/eLife.07051.

Saper CB, Fuller PM, Pedersen NP, Lu J \& Scammell TE. (2010). Sleep state switching. Neuron 68, 1023-1042.

Schlaefke ME, See WR \& Loeschcke HH. (1970). Ventilatory response to alterations of $\mathrm{H}+$ ion concentration in small areas of the ventral medullary surface. RespirPhysiol 10, 198-212.

Schreihofer AM \& Guyenet PG. (1997). Identification of C1 presympathetic neurons in rat rostral ventrolateral medulla by juxtacellular labeling in vivo. Journal of Comparative Neurology 387, 524-536.

Semenza GL. (2012). Hypoxia-inducible factors in physiology and medicine. Cell 148, 399-408.

Severinghaus JW. (1998). Hans Loeschcke, Robert Mitchell and the medullary CO2 chemoreceptors: a brief historical review. RespirPhysiol 114, 17-24.

Smith CA, Forster HV, Blain GM \& Dempsey JA. (2010). An interdependent model of central/peripheral chemoreception: Evidence and implications for ventilatory control. Respiratory physiology \& neurobiology 173, 288-297.

Smith CA, Henderson KS, Xi L, Chow C-M, Eastwood PR \& Dempsey JA. (1997). Neuralmechanical coupling of breathing in REM sleep. J App/ Physiol 83, 1923-1932. 
Smith CA, Rodman JR, Chenuel BJ, Henderson KS \& Dempsey JA. (2006). Response time and sensitivity of the ventilatory response to $\mathrm{CO} 2$ in unanesthetized intact dogs: central vs. peripheral chemoreceptors. J Appl Physiol 100, 13-19.

Smith JC, Abdala AP, Borgmann A, Rybak IA \& Paton JF. (2013). Brainstem respiratory networks: building blocks and microcircuits. Trends Neurosci 36, 152-162.

Smith JC, Morrison DE, Ellenberger HH, Otto MR \& Feldman JL. (1989). Brainstem projections to the major respiratory neuron populations in the medulla of the cat. $J$ Comp Neurol 281, 69-96.

Sparta DR, Stamatakis AM, Phillips JL, Hovelso N, van ZR \& Stuber GD. (2012). Construction of implantable optical fibers for long-term optogenetic manipulation of neural circuits. Nat Protoc 7, 12-23.

Stornetta RL, Moreira TS, Takakura AC, Kang BJ, Chang DA, West GH, Brunet JF, Mulkey DK, Bayliss DA \& Guyenet PG. (2006). Expression of Phox $2 b$ by brainstem neurons involved in chemosensory integration in the adult rat. J Neurosci 26, 10305-10314.

Stornetta RL, Spirovski D, Moreira TS, Takakura AC, West GH, Gwilt JM, Pilowsky PM \& Guyenet PG. (2009). Galanin is a selective marker of the retrotrapezoid nucleus in rats. J Comp Neurol 512, 373-383.

Strohl KP, Thomas AJ, St Jean P, Schlenker EH, Koletsky RJ \& Schork NJ. (1997). Ventilation and metabolism among rat strains. J Appl Physiol (1985) 82, 317-323.

Sullivan CE, Murphy E, Kozar LF \& Phillipson EA. (1979). Ventilatory responses to CO2 and lung inflation in tonic versus phasic REM sleep. J Appl Physiol 47, 1305-1310.

Sun MK \& Reis DJ. (1993). Differential responses of barosensitive neurons of rostral ventrolateral medulla to hypoxia in rats. Brain Res 609, 333-337.

Sun MK \& Reis DJ. (1994). Central neural mechanisms mediating excitation of sympathetic neurons by hypoxia. Prog in Neurobiol 44, 197-219.

Sun QJ, Berkowitz RG \& Pilowsky PM. (2008). GABA A mediated inhibition and post-inspiratory pattern of laryngeal constrictor motoneurons in rat. Respiratory physiology \& neurobiology 162, 41-47. 
Sun QJ, Llewellyn-Smith I, Minson J, Arnolda L, Chalmers J \& Pilowsky P. (1996). Thyrotropinreleasing hormone immunoreactive boutons form close appositions with medullary expiratory neurons in the rat. Brain Res 715, 136-144.

Takakura AC, Moreira TS, Colombari E, West GH, Stornetta RL \& Guyenet PG. (2006). Peripheral chemoreceptor inputs to retrotrapezoid nucleus (RTN) CO2-sensitive neurons in rats. $J$ Physiol 572, 503-523.

Takakura AC, Moreira TS, Stornetta RL, West GH, Gwilt JM \& Guyenet PG. (2008a). Selective lesion of retrotrapezoid Phox $2 \mathrm{~b}$-expressing neurons raises the apnoeic threshold in rats. J Physiol 586, 2975-2991.

Takakura AC, Moreira TS, Stornetta RL, West GH, Gwilt JM \& Guyenet PG. (2008b). Selective lesion of retrotrapezoid Phox $2 \mathrm{~b}$-expressing neurons raises the apnoeic threshold in rats. J Physiol 586, 2975-2991.

Takakura AC, Moreira TS, West GH, Gwilt JM, Colombari E, Stornetta RL \& Guyenet PG. (2007). GABAergic pump cells of solitary tract nucleus innervate retrotrapezoid nucleus chemoreceptors. J Neurophysiol 98, 374-381.

Tan EM, Yamaguchi Y, Horwitz GD, Gosgnach S, Lein ES, Goulding M, Albright TD \& Callaway EM. (2006). Selective and quickly reversible inactivation of mammalian neurons in vivo using the Drosophila allatostatin receptor. Neuron 51, 157-170.

Teppema LJ \& Dahan A. (2010). The ventilatory response to hypoxia in mammals: mechanisms, measurement, and analysis. Physiol Rev 90, 675-754.

Timmers HJ, Wieling W, Karemaker JM \& Lenders JW. (2003). Denervation of carotid baro- and chemoreceptors in humans. J Physiol 553, 3-11.

Tupal S, Huang WH, Picardo MC, Ling GY, Del Negro CA, Zoghbi HY \& Gray PA. (2014). Atoh1dependent rhombic lip neurons are required for temporal delay between independent respiratory oscillators in embryonic mice. elife 3, e02265.

Veasey SC, Fornal CA, Metzler CW \& Jacobs BL. (1995). Response of serotonergic caudal raphe neurons in relation to specific motor activities in freely moving cats. Journal of Neuroscience 15, 5346-5359. 
Verna A. (1979). Ulstrastructure of the carotid body in the mammals. Int Rev Cytol 60, 271-330.

Wakai J, Takamura D, Morinaga R, Nakamuta N \& Yamamoto Y. (2015). Differences in respiratory changes and Fos expression in the ventrolateral medulla of rats exposed to hypoxia, hypercapnia, and hypercapnic hypoxia. Respir Physiol Neurobiol 215, 64-72.

Wang S, Benamer N, Zanella S, Kumar NN, Shi Y, Bevengut M, Penton D, Guyenet PG, Lesage F, Gestreau C, Barhanin J \& Bayliss DA. (2013a). TASK-2 channels contribute to $\mathrm{pH}$ sensitivity of retrotrapezoid nucleus chemoreceptor neurons. J Neurosci 33, 1603316044.

Wang S, Benamer N, Zanella S, Kumar NN, Shi Y, Bevengut M, Penton D, Guyenet PG, Lesage F, Gestreau C, Barhanin J \& Bayliss DA. (2013b). TASK-2 channels contribute to pH sensitivity of retrotrapezoid nucleus chemoreceptor neurons. J Neurosci 33, 1603316044.

Wang S, Shi Y, Shu S, Guyenet PG \& Bayliss DA. (2013c). Phox2b-expressing retrotrapezoid neurons are intrinsically responsive to acidification and $\mathrm{CO}_{2}$. Journal of Neuroscience 33, 7756-7761.

Weese-Mayer DE, Berry-Kravis EM, Ceccherini I, Keens TG, Loghmanee DA \& Trang H. (2010). An official ATS clinical policy statement: Congenital central hypoventilation syndrome: genetic basis, diagnosis, and management. Am J Respir Crit Care Med 181, 626-644.

Weese-Mayer DE, Rand CM, Berry-Kravis EM, Jennings LJ, Loghmanee DA, Patwari PP \& Ceccherini I. (2009). Congenital central hypoventilation syndrome from past to future: model for translational and transitional autonomic medicine. PediatrPulmonol 44, 521535.

Weil JV. (2004). Sleep at high altitude. High altitude medicine \& biology 5, 180-189.

Weston MC, Stornetta RL \& Guyenet PG. (2004). Glutamatergic neuronal projections from the marginal layer of the rostral ventral medulla to the respiratory centers in rats. Journal of Comparative Neurology 473, 73-85.

Yokota S, Oka T, Tsumori T, Nakamura S \& Yasui Y. (2007). Glutamatergic neurons in the KollikerFuse nucleus project to the rostral ventral respiratory group and phrenic nucleus: a 
combined retrograde tracing and in situ hybridization study in the rat. Neurosci Res 59, 341-346. 